\title{
Drift velocity measurements for the CRIPT prototype drift chamber tracking system
}

by

\author{
Andrew Erlandson \\ A Thesis submitted to \\ the Faculty of Graduate and Postdoctoral Affairs \\ in partial fulfilment of \\ the requirements for the degree of \\ Master of Science \\ Ottawa-Carleton Institute for \\ Physics
}

Department of Physics

Carleton University

Ottawa, Ontario, Canada

August 2011

Copyright (C)

2011 - Andrew Erlandson 
Library and Archives

Canada

Published Heritage

Branch

395 Wellington Street Ottawa ON K1A 0N4 Canada
Bibliothèque et

Archives Canada

Direction du

Patrimoine de l'édition

395 , rue Wellington

Ottawa ON K1A ON4

Canada
Your file Votre référence
ISBN: $978-0-494-83196-0$
Our file Notre référence
ISBN: $978-0-494-83196-0$

\section{NOTICE:}

The author has granted a nonexclusive license allowing Library and Archives Canada to reproduce, publish, archive, preserve, conserve, communicate to the public by telecommunication or on the Internet, loan, distribute and sell theses worldwide, for commercial or noncommercial purposes, in microform, paper, electronic and/or any other formats.

The author retains copyright ownership and moral rights in this thesis. Neither the thesis nor substantial extracts from it may be printed or otherwise reproduced without the author's permission.
AVIS:

L'auteur a accordé une licence non exclusive permettant à la Bibliothèque et Archives Canada de reproduire, publier, archiver, sauvegarder, conserver, transmettre au public par télécommunication ou par l'Internet, prêter, distribuer et vendre des thèses partout dans le monde, à des fins commerciales ou autres, sur support microforme, papier, électronique et/ou autres formats.

L'auteur conserve la propriété du droit d'auteur et des droits moraux qui protège cette thèse. $\mathrm{Ni}$ la thèse ni des extraits substantiels de celle-ci ne doivent être imprimés ou autrement reproduits sans son autorisation.
In compliance with the Canadian Privacy Act some supporting forms may have been removed from this thesis.

While these forms may be included in the document page count, their removal does not represent any loss of content from the thesis.
Conformément à la loi canadienne sur la protection de la vie privée, quelques formulaires secondaires ont été enlevés de cette thèse.

Bien que ces formulaires aient inclus dans la pagination, il n'y aura aucun contenu manquant.

\section{Canadä}




\section{Abstract}

The constantly changing political climate, catalysed by the September 11th terrorist attacks, has among other things made it necessary to strictly monitor the contents of imported and exported cargo. The CRIPT collaboration was born out of a CRTI grant which aims to construct a tomography apparatus to effectively image the contents of sea containers using high energy cosmic ray muons. The portion of the research delegated to Carleton University involves the determination of the suitability of large area drift chambers to meet the tomographic requirements of the collaboration.

The purpose of this thesis is to examine the drift velocity of ionization in the small scale prototype drift chambers. In order to accomplish this goal an in-depth treatment of both physics and instrumentation is necessary. A structure was built and designed to accommodate the prototype tracking system along with all necessary software to make data analysis possible. Candidate drift gases such as argon-methane, argonethane, argon-carbon dioxide, and argon-ethane-acetylene were scrutinized with various measurement techniques to understand the quality of their performance in the prototype detectors. The experimental results were compared to Monte Carlo data calculated with MAGBOLTZ and a successive over relaxation (SOR) simulation and good agreement was found. Where agreement was not found it was hypothesized that water vapour contaminated the drift volume. This hypothesis was verified with MAGBOLTZ simulations and provides adequate reason to believe water vapour is the source of the problem. This research provides a means to verify the drift velocity 
inside the detectors which is a necessary parameter to know in order to completely understand the performance of the detectors themselves. 
This thesis is dedicated to my good friend caffeine - without whom none of this would have been possible 


\section{Acknowledgments}

I would like to thank the following people for their support, advice, and most importantly sense of humour over the course of my studies at Carleton University.

First and foremost I would like to thank my thesis supervisor Dr. John Armitage and his endless patience in helping me learn over the course of these past two years. I have learnt more in this time than in the entirety of my undergraduate degree. I feel that I have come one step closer to becoming a true scientist. I am now an appreciator for all the very hard work that must be put into bringing ideas from the blackboard to the laboratory which is seldom as easy as it sounds. I thank John for his understanding as I slowly came to learn the skills required to complete this degree. I would also like to thank him for the opportunity to work with the CRIPT collaboration which has been a fantastic experience.

I would also like to thank Dr. Khalil Boudjemline, the Carleton CRIPT postdoctoral research associate, for his help in familiarizing me with the tools necessary to work directly with drift chambers. His work with the SOR simulation and the data analysis code allowed me to direct my focus on my own work and for that I am exceptionally grateful. I would also like to thank him for answering my many late night e-mails as inspiration always seems to strike at the least convenient times.

I must also thank James Botte, the undergraduate research assistant, for his expertise in designing the DAQ system which was also of critical importance to the work presented here. 
I would also like to thank all of the co-op students that have worked with our group at Carleton University. Good help is exceedingly hard to find but when it is found much can be accomplished.

I must thank Philippe Gravelle and Yves Baribeau for their technical expertise and flexibility in helping me solve problems ranging from building a structure to support the prototype detectors to soldering seemingly impossible things back together.

I would like to thank the Department of Physics at Carleton University for making my studies as straightforward as possible.

I would like to thank all of my office mates, both past and present, who have provided not only stimulating academic discussions but also much needed humorous conversation. I also appreciate the friendships that have stemmed from all of my interactions with my colleagues over the course of my graduate studies.

From outside of the academic world I would like to thank my parents Bob and Barb Erlandson for their understanding in my erratic work schedule and for taking care of my cat Gary as I spent more waking hours at the university than anywhere else. I would like to thank my sister Leah all of the free coffee she was able to provide me.

I would also like to thank my friends from outside of the university. Their willingness to listen to my droning about the subtleties of working with prototype particle detectors did not go unnoticed. They also provided valuable distractions from the swirling vortex of entropy that is my office desk. 


\section{Table of Contents}

$\begin{array}{ll}\text { Abstract } & \text { ii }\end{array}$

$\begin{array}{lll}\text { Acknowledgments } & \text { v }\end{array}$

Table of Contents $\quad$ vii

List of Tables $\quad$ xi

List of Figures $\quad$ xiii

Nomenclature $\quad$ xx

1 CRIPT: Cosmic Ray Inspection and Passive Tomography 1

1.1 Introduction . . . . . . . . . . . . . . . . . 1

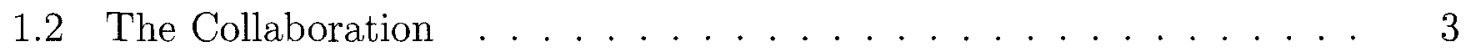

1.3 National Security . . . . . . . . . . . . . . . 4

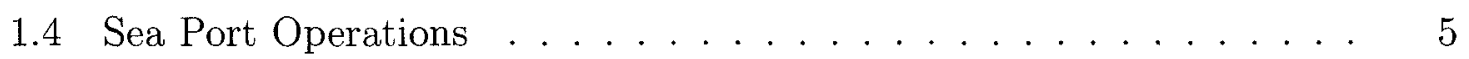

1.5 Muon Tomography ........................ 6

1.6 Review of similar projects . . . . . . . . . . . . . 7

1.6.1 Author's contribution ............... 9

2 Physics for Muon Detection in Drift Chambers 11

2.1 Cosmic Rays. . . . . . . . . . . . . . . . . 11 
2.2 Atmospheric interactions . . . . . . . . . . . . . . . . . 14

2.3 The Muon . . . . . . . . . . . . . . . . . . . . . . 16

2.3 .1 Cosmic ray muon flux f . . . . . . . . . . . 16

2.3 .2 Special Relativity . . . . . . . . . . . . . . 20

2.3 .3 Interaction with matter . . . . . . . . . . . . . 21

2.3.4 Radiation Length . . . . . . . . . . . . . . . . . . 24

2.3 .5 Multiple Scattering . . . . . . . . . . . . . 25

2.4 Instrumentation . . . . . . . . . . . . . . . 26

2.4 .1 Scintillation detectors . . . . . . . . . . . . . 27

2.5 Ionization . . . . . . . . . . . . . . . . . . . 36

2.5.1 Electron transport . . . . . . . . . . . . . . 40

3 CRIPT prototypes $\quad 45$

3.1 Small-scale prototypes . . . . . . . . . . . . . . 45

3.1.1 High voltage $(\mathrm{HV})$ electronics $\ldots \ldots \ldots \ldots . \ldots . \ldots$

3.1.2 Signal Processing and Readout Electronics . . . . . . . . . 53

3.2 Drift gases . . . . . . . . . . . . . . . . 61

3.3 Drift chamber simulation . . . . . . . . . . . . . . . . 64

4 Experimental techniques $\quad 71$

4.1 Geometrical Discrimination . . . . . . . . . . . . . . . 71

4.2 Active Area Reduction . . . . . . . . . . . . . . . 77

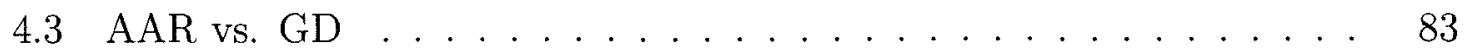

$4.3 .1 \quad \mathrm{Ar}^{-\mathrm{CH}_{4}} \ldots \ldots \ldots \ldots \ldots \ldots \ldots \ldots$

$4.3 .2 \quad \mathrm{Ar}-\mathrm{CO}_{2} \ldots \ldots \ldots \ldots \ldots \ldots$

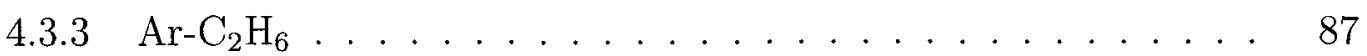

4.3 .4 Choice of method . . . . . . . . . . . . . . . 89 


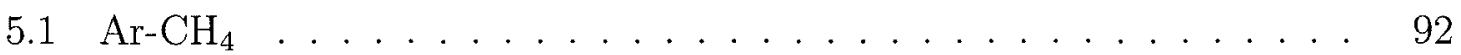

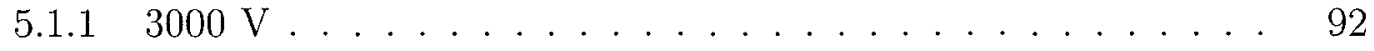

$5.1 .24000 \mathrm{~V} \ldots \ldots \ldots \ldots \ldots \ldots \ldots \ldots$

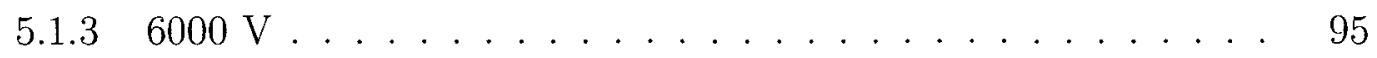

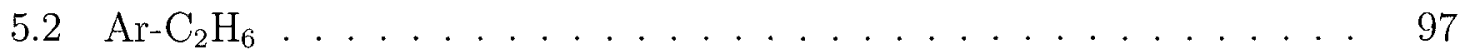

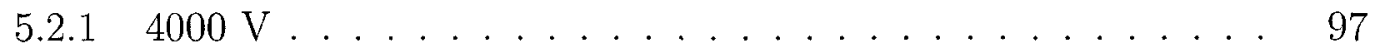

$5.2 .26000 \mathrm{~V} \ldots \ldots \ldots \ldots \ldots \ldots \ldots \ldots$

$5.3 \quad \mathrm{Ar}^{-} \mathrm{C}_{2} \mathrm{H}_{2}-\mathrm{C}_{2} \mathrm{H}_{6} \ldots \ldots \ldots \ldots \ldots \ldots \ldots$

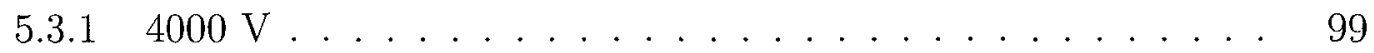

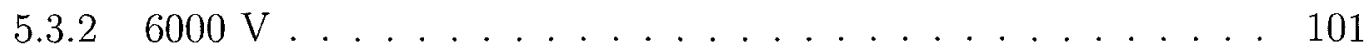

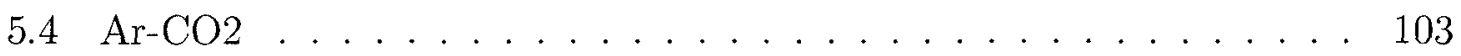

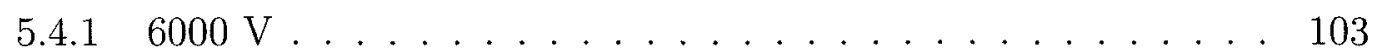

5.5 Summary of results . . . . . . . . . . . . . . 103

5.6 Effects of $\mathrm{H}_{2} \mathrm{O}$ contamination $\ldots \ldots \ldots \ldots \ldots \ldots$

$5.6 .1 \quad \mathrm{Ar}^{-\mathrm{CH}_{4}} \ldots \ldots \ldots \ldots \ldots \ldots \ldots$

$5.6 .2 \quad \mathrm{Ar}_{-} \mathrm{C}_{2} \mathrm{H}_{2}-\mathrm{C}_{2} \mathrm{H}_{6} \ldots \ldots \ldots \ldots \ldots \ldots \ldots \ldots$

5.6 .3 Conclusion . . . . . . . . . . . . . . . . . 114

5.7 Experimental error analysis . . . . . . . . . . . . . . 114

$\begin{array}{lll}6 & \text { Summary } & 119\end{array}$

$\begin{array}{ll}\text { References } & 121\end{array}$

Appendix A Further investigation of $\mathrm{H}_{2} \mathrm{O}$ contamination with MAGBOLTZ

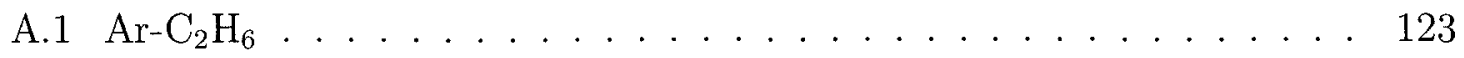

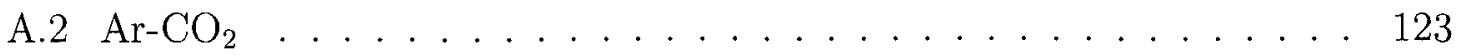


Appendix B Investigation of air contamination with MAGBOLTZ 127

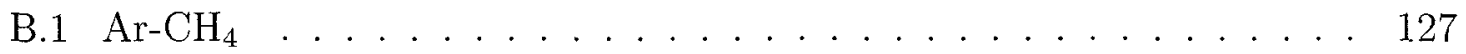

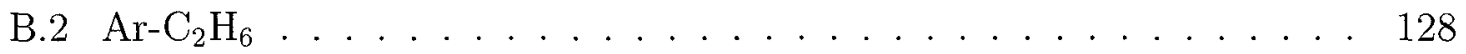

B.3 $\mathrm{Ar}_{-} \mathrm{C}_{2} \mathrm{H}_{6}-\mathrm{C}_{2} \mathrm{H}_{2} \ldots \ldots \ldots \ldots \ldots \ldots \ldots \ldots \ldots \ldots \ldots \ldots \ldots \ldots \ldots \ldots \ldots$

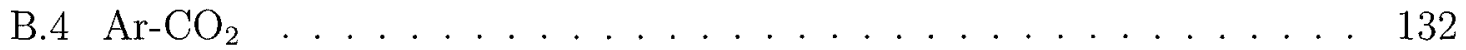

B.5 Conclusion . . . . . . . . . . . . . . . . . . . 132

$\begin{array}{ll}\text { Appendix C Humidity } & 134\end{array}$

C.1 Relative Humidity . . . . . . . . . . . . . . . . . . . . . 134 


\section{List of Tables}

2.1 Variables used in the Bethe-Bloch equation. . . . . . . . . . . 23

2.2 Values for $\mathrm{L}_{\text {rad }}$ and $\mathrm{L}_{\text {rad }}^{\prime}$. All quantities here are dimensionless. . . . . 25

4.1 Summary of both simulated and experimental data in $\mathrm{Ar}-\mathrm{CH}_{4}$. The units of drift velocity are $\mu \mathrm{m} / \mathrm{ns} . \ldots \ldots \ldots \ldots$

4.2 Summary of $\chi_{r e d}^{2}$ for P10 gas. . . . . . . . . . . . . 85

4.3 Summary of both simulated and experimental data in $\mathrm{Ar}-\mathrm{CO}_{2}$. The units of drift velocity are $\mu \mathrm{m} / \mathrm{ns} . \ldots \ldots \ldots \ldots . \ldots . \ldots 87$

4.4 Summary of $\chi_{r e d}^{2}$ for Ar- $\mathrm{CO}_{2}$ gas. . . . . . . . . . . 87

4.5 Summary of both simulated and experimental data in $\mathrm{Ar}-\mathrm{C}_{2} \mathrm{H}_{6}$. The units of drift velocity are $\mu \mathrm{m} / \mathrm{ns} . \ldots \ldots \ldots . \ldots 8$

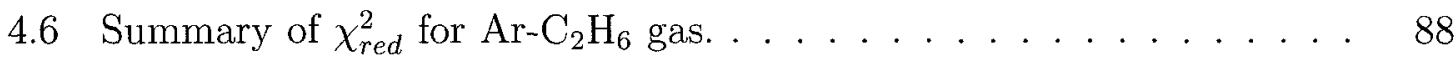

5.1 Summary of experimental results for P10 at $3000 \mathrm{~V}$ on the cathode. The units of drift velocity are $\mu \mathrm{m} / \mathrm{ns} \ldots \ldots \ldots \ldots 2$

5.2 Summary of $\chi_{\text {red }}^{2}$ for Ar- $\mathrm{CH}_{4}$ gas. . . . . . . . . . . . . . 92

5.3 Summary of experimental results for trimix at $4 \mathrm{kV}$ and $6 \mathrm{kV}$ on the cathode. The units of drift velocity are $\mu \mathrm{m} / \mathrm{ns} \ldots \ldots \ldots$. . . . . 102

5.4 Summary of $\chi_{\text {red }}^{2}$ for trimix gas. . . . . . . . . . . . . . . 102 
5.5 Summary of experimental results. The units of drift velocity are $\mu \mathrm{m} / \mathrm{ns}$. The cathode voltages can be expressed in terms of their resulting drift fields in the following way: $3000 \mathrm{~V}$ corresponds to 52.6 $\mathrm{V} / \mathrm{cm}, 4000 \mathrm{~V}$ corresponds to $70.2 \mathrm{~V} / \mathrm{cm}$, and $6000 \mathrm{~V}$ corresponds to $105.2 \mathrm{~V} / \mathrm{cm} \ldots \ldots \ldots \ldots \ldots \ldots \ldots \ldots$

5.6 Summary of experimental results by approximate date of data collection.104

5.7 Summary of experimental results for P10 with $3 \mathrm{kV}$ on the cathode with $0.1 \%$ contamination with $\mathrm{H}_{2} \mathrm{O}$. The units of drift velocity are

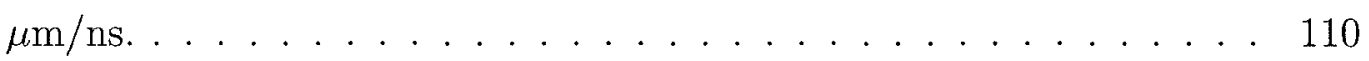

5.8 Summary of experimental results for trimix at $4000 \mathrm{~V}$ on the cathode with $0.3 \%$ contamination with $\mathrm{H}_{2} \mathrm{O}$. The units of drift velocity are $\mu \mathrm{m} / \mathrm{ns} .111$

5.9 Summary of experimental results for trimix at $6000 \mathrm{~V}$ on the cathode with $0.5 \%$ contamination with $\mathrm{H}_{2} \mathrm{O}$. The units of drift velocity are $\mu \mathrm{m} / \mathrm{ns} .113$

5.10 Summary of the sources of error . . . . . . . . . . . . . . 114

B.1 Summary of conamination results for water and air in all gases. The values of drift velocity are quotes from the MAGBOLTZ plots at a drift field of $105.2 \mathrm{~V} / \mathrm{cm}$. The units of drift velocity are $\mu \mathrm{m} / \mathrm{ns} . \ldots . .132$ 


\section{List of Figures}

1.1 Layout of a generic Canadian sea port [1]. Used with permission. . . 5

1.2 Cross section of CRIPT MT system . . . . . . . . . . . . 7

2.1 Cosmic ray muon spectrum for two angles. The curve with mostly solid data points is for $0^{\circ}$ whereas the second curve (hollow triangles) is for incident muons at an angle of $75^{\circ}$. [2] . . . . . . . . . . 13

2.2 Simplified high altitude proton/pion decay. [3] . . . . . . . . . . 16

2.3 The standard model of particle physics. [3] . . . . . . . . . . . 17

2.4 Fit to data collected by the BESS experiment. Note: Differential flux is in units of $m^{-2} s r^{-1} s^{-1}(G e V / c)^{-1}$. [4] . . . . . . . . . 18

2.5 Muon interaction with copper [2] . . . . . . . . . . . . 21

2.6 Light guide from scintillator side to square face. . . . . . . . . . . . 30

2.7 Cylindrical light guide . . . . . . . . . . . . . . . . 30

2.8 Mock-up of light collection system . . . . . . . . . . . . . 31

2.9 Trigger scintillator actually used for this thesis. . . . . . . . . . . 32

2.10 Trigger logic circuit . . . . . . . . . . . . . . . . . 33

2.11 Triple rate curves . . . . . . . . . . . . . . . . . 35 
3.1 Exploded view of CRIPT prototype. Constituents and their thicknesses are listed along with 2 spatial dimensions. The gas flows in the $1.5 \mathrm{~cm}$ tall region between the two cathode planes. The quantities on the right denote the thickness of layers they pertain to. The correlation between material and quantity is by colour code. . . . . . . . . 46

3.2 Close-up of CRIPT zig-zag cathode board. . . . . . . . . . . . 48

3.3 Engineering drawing of the deflection experienced by a large scale drift chamber lid at atmospheric pressure. $[5] \ldots \ldots \ldots \ldots$

3.4 Simplified high voltage board diagram $\ldots \ldots \ldots . \ldots . \ldots$

3.5 Photo of actual high voltage ladders. Visible are both connections for upper and lower cathode boards and $1 \mathrm{M} \Omega$ resisters on circuit wafer.

3.6 Photo of actual preamplifier board. Cremat-112 preamplifiers were used for the research in this thesis . . . . . . . . . . . . .

3.7 A typical pulse from the CRIPT prototype drift chamber preamplifiers. Notice the fast rise and slow decay of the pulse. This is due to the choice of preamplifiers . . . . . . . . . . . . . .

3.8 CRIPT cosmic ray muon trigger system with drift chamber location shown. . . . . . . . . . . . . . . . .

3.9 Geometrical MC results for tracking muons through three layers of scintillators separated by the distances previously listed. Lower right corner: Blue is the top scintillator, red is the middle, and black is the bottom scintillator's momentum spectrum . . . . . . . . . . . .

3.10 Top: Hits in both $\mathrm{X}$ and $\mathrm{Y}$ with frequency denoted by colour. The most probable hit position region is in the center of the drift area. Middle/Bottom: Projections of the top plot onto the $\mathrm{X}$ and $\mathrm{Y}$ axis

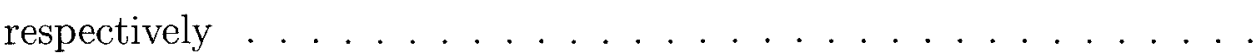


3.11 Transverse diffusion (top), longitudinal diffusion (middle), and drift velocity (bottom) from MAGBOLTZ . . . . . . . . . .

3.12 Successive Over Relaxation (SOR) results for the electric field inside a CRIPT drift chamber. Bottom: Field lines resulting from the SOR calculation. The choice of axis is arbitrary for this diagram. As a point of reference the anode wire would run into and out of the page. The units for the colour scale are $\mathrm{V} / \mathrm{cm} \ldots \ldots \ldots \ldots \ldots$

3.13 Close-up of anode wire and resulting field. This is the gas gain region where ionization avalanches occur. The units for the colour scale are

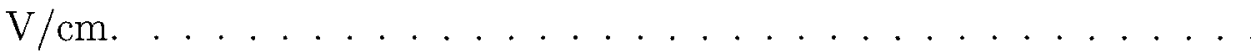

3.14 An enhanced view of how the electric field changes when very close to the gaps in the cathode boards. The units for the colour scale are $\mathrm{V} / \mathrm{cm} .68$

3.15 Simulated flight path for an electron near the anode plane. . . . . . 69

3.16 Simulated flight path for an electron near the cathode board. . . . . . 69

3.17 SOR and MAGBOLTZ results for drift velocity in the CRIPT prototypes. Different gases are denoted by different colours. $\mathrm{ArCH}_{4}$ is in black, $\mathrm{ArC}_{2} \mathrm{H}_{6}$ is in red, $\mathrm{ArC}_{2} \mathrm{H}_{2} \mathrm{C}_{2} \mathrm{H}_{6}$ is in blue, and $\mathrm{ArCO}_{2}$ is in green. The marker shapes denote the applied cathode voltage. Squares are $6000 \mathrm{~V}$ and triangles are $4000 \mathrm{~V} . \ldots \ldots \ldots \ldots$

4.1 A cross-sectional view of the experimental apparatus. Shown are the overlap of the two top most scintillators, half of the detector, and the other two trigger scintillators. The entirety of both top scintillators is not shown because the area that is not used in the overlap does not contribute to the illumination of the detector. . . . . . . . . . .

4.2 An example of a typical timing distribution obtained from the CRIPT drift chambers and DAQ. The distribution is the flight time of the fastest ionization to create a signal on the anode wire. . . . . . . . . 
4.3 Simulated GD timing histogram. Events are projected into the plane of the anode wire and the mean can be extracted. . . . . . . . 75

4.4 An example of a typical timing distribution obtained from the implementation of the GD method.

4.5 Top: 500 events are generated for each data point. Middle: 1000 events are generated for each data point. Bottom: 2000 events are generated for each data point. . . . . . . . . . . . . . . 76

4.6 Cross-sectional view of the experimental apparatus utilizing the AAR method

4.7 A simulated timing distribution for the AAR method. . . . . . . .

4.8 An actual timing histogram with fitting and correction to ' $\mathrm{t}_{\max }$ ' present from the AAR method. . . . . . . . . . . . . . . . . .

4.9 The CRIPT drift chamber tracking system in the prototype stage. Shown are the four prototypes. The top three are small scale and bottom is the full sized drift chamber. The drift chambers can be identified by the shielding covering their preamplifier/HV boards. In the photo this appears as the off-white rectangles in the centre of the

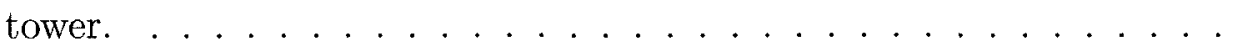

4.10 GD and AAR data plotted linearly along with the Monte Carlo for $4000 \mathrm{~V}$ applied to the cathode and $5820 \mathrm{~V}$ applied to the anode. The error bars on the GD data points are too small to be seen. Note: P10 is the common name for $\mathrm{Ar}^{-} \mathrm{CH}_{4} \ldots \ldots \ldots \ldots \ldots$

4.11 GD and AAR data plotted linearly along with the Monte Carlo for $6000 \mathrm{~V}$ applied to the cathode and $7820 \mathrm{~V}$ applied to the cathode. . .

4.12 GD and AAR data plotted linearly along with the Monte Carlo for $6000 \mathrm{~V}$ applied to the cathode and $8000 \mathrm{~V}$ applied to the anode. . . . 
4.13 GD and AAR hybrid data plotted linearly along with the Monte Carlo for $4000 \mathrm{~V}$ applied to the cathode and $5590 \mathrm{~V}$ applied to the anode. . $\quad 88$

4.14 GD and AAR hybrid data plotted linearly along with the Monte Carlo for $6000 \mathrm{~V}$ applied to the cathode and $7590 \mathrm{~V}$ applied to the anode. .

5.1 Drift velocity curve for $3000 \mathrm{~V}$. These data were likely taken after

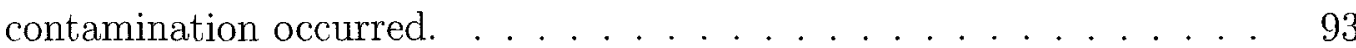

5.2 Linear plot for $3000 \mathrm{~V}$. Note: Error bars on the experimental data are too small to be seen. These data were likely taken after contamination

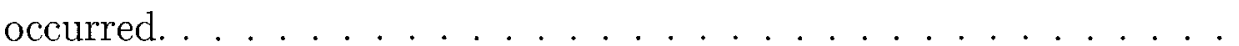

5.3 Drift velocity curve for $4000 \mathrm{~V}$. . . . . . . . . . . . . . . 94

5.4 Drift velocity curve for $6000 \mathrm{~V}$. . . . . . . . . . . . . 95

5.5 MAGBOLTZ results for all gases used in this research shown for a larger range of electric field strengths. The drift velocity is shown on the bottom plot. ................... 96

5.6 Drift velocity curve for $4000 \mathrm{~V}$. . . . . . . . . . . . . 98

5.7 Drift velocity curve for $6000 \mathrm{~V} \ldots \ldots 9$

5.8 Drift velocity curve for $4000 \mathrm{~V}$. These data were likely taken after contamination occurred. . . . . . . . . . . . . 100

5.9 Linear plot for $4000 \mathrm{~V}$. These data were likely taken after contamination occurred.

5.10 Drift velocity curve for $6000 \mathrm{~V}$. These data were likely taken after contamination occurred.

5.11 Linear plot for $6000 \mathrm{~V}$. These data were likely taken after contamination occurred.

5.12 Drift velocity curve for $6000 \mathrm{~V}$. . . . . . . . . . . . . . 103

5.13 MAGBOLTZ results for $\mathrm{H}_{2} \mathrm{O}$ contamination in $\mathrm{ArCH}_{4}$. Top: Transverse diffusion. Middle: Longitudinal diffusion. Bottom: Drift velocity. 107 
5.14 MAGBOLTZ results for $\mathrm{H}_{2} \mathrm{O}$ contamination in $\mathrm{ArC}_{2} \mathrm{H}_{2} \mathrm{C}_{2} \mathrm{H}_{6}$. Top: Transverse diffusion. Middle: Longitudinal diffusion. Bottom: Drift velocity. . . . . . . . . . . . . . . . . . . 108

5.15 Drift velocity curve for argon-methane at $3000 \mathrm{~V}$ on the cathode with $0.1 \% \mathrm{H}_{2} \mathrm{O}$ vapour present. . . . . . . . . . . . . 109

5.16 Linear plot for argon-methane at $3000 \mathrm{~V}$ on the cathode with $0.1 \%$ $\mathrm{H}_{2} \mathrm{O}$ vapour present. . . . . . . . . . . . . . 110

5.17 Drift velocity curve for trimix at $4000 \mathrm{~V}$ on the cathode with $0.3 \%$ $\mathrm{H}_{2} \mathrm{O}$ vapour present. The vertical scale is plotted on a $\log$ axis. . . .

5.18 Linear plot for trimix at $4000 \mathrm{~V}$ on the cathode with $0.3 \% \mathrm{H}_{2} \mathrm{O}$ vapour present.

5.19 Drift velocity curve for trimix at $6000 \mathrm{~V}$ on the cathode with $0.5 \%$

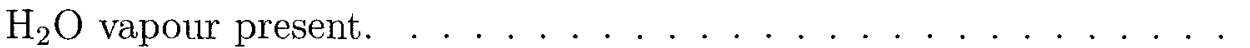

5.20 Linear plot for trimix at $6000 \mathrm{~V}$ on the cathode with $0.5 \% \mathrm{H}_{2} \mathrm{O}$ vapour present.

A.1 MAGBOLTZ results for argon-ethane contaminated with water. Top: Transverse diffusion. Middle: Longitudinal diffusion. Bottom: Drift velocity . . . . . . . . . . . . . . . . .

A.2 MAGBOLTZ results for argon-carbon dioxide contaminated with water. Top: Transverse diffusion. Middle: Longitudinal diffusion. Bottom: Drift velocity . . . . . . . . . . . .

B.1 MAGBOLTZ results for argon-methane contaminated with air. Top: Transverse diffusion. Middle: Longitudinal diffusion. Bottom: Drift

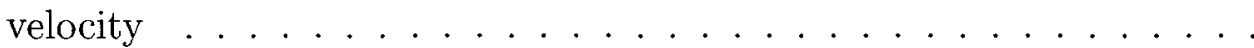

B.2 MAGBOLTZ results for argon-ethane contaminated with air. Top: Transverse diffusion. Middle: Longitudinal diffusion. Bottom: Drift velocity $\ldots \ldots \ldots \ldots \ldots \ldots \ldots \ldots$ 
B.3 MAGBOLTZ results for argon-ethane-acetylene contaminated with air. Top: Transverse diffusion. Middle: Longitudinal diffusion. Bottom: Drift velocity ....................... . . 131

B.4 MAGBOLTZ results for argon-carbon dioxide contaminated with air. Top: Transverse diffusion. Middle: Longitudinal diffusion. Bottom: Drift velocity . . . . . . . . . . . . . . . . 133

C.1 Relative humidity from Jan. 2011 to June 2011 . . . . . . . . . . . . 135

C.2 Mean temperature from Jan. 2011 to June 2011 . . . . . . . . . . . 136 


\section{Nomenclature}

\section{Table of Abbreviations}

This thesis contains a number of commonly used abbreviations within the field of detector development; and in order to make the thesis more inclusive, a list of prevalent abbreviations can be seen below:

\begin{tabular}{cc}
\hline \hline Abbreviation & Definition \\
\hline CRIPT & Cosmic Ray Inspection and Passive Tomography \\
MT & Muon Tomography \\
AECL & Atomic Energy of Canada Limited \\
CBSA & Canada Border Services Agency \\
DRDC & Defence Research and Development Canada \\
ISR & International Safety Research \\
AAPS & Advanced Applied Physics Solutions \\
CRTI & Canadian Research and Technology Iinitiative \\
SNM & Special Nuclear Material
\end{tabular}




\section{Table of Symbols}

This thesis uses a number of symbols which are application specific, and can be the cause of ambiguity. This list is provided to help combat that ambiguity:

\begin{tabular}{|c|c|}
\hline Symbol & Definition \\
\hline$c$ & speed of light $\left(2.998 \times 10^{8} \mathrm{~m} / \mathrm{s}\right)$ \\
\hline$e$ & Electron charges $\left(-1.602 \times 10^{-19} \mathrm{C}\right)$ \\
\hline$E$ & Energy, usually in units of electron-Volts \\
\hline$\beta$ & Relativistic quantity $v / c$ \\
\hline$\gamma$ & Relativistic quantity, $E / m c^{2}$ \\
\hline$\frac{d E}{d x}$ & Energy deposition in a medium per unit length \\
\hline$\left\langle E_{d r i f t}\right\rangle$ & Electric field in a Drift Chamber, $V / \mathrm{cm}$ \\
\hline$Z$ & Atomic number of an atom \\
\hline $\mathrm{X}_{0}$ & Radiation length \\
\hline$\Theta_{0}$ & Mutliple scattering width \\
\hline$R(E)$ & Range of primary radiation \\
\hline$V_{d r \imath f t}$ & Macroscopic drift velocity \\
\hline$\left\langle E_{\text {draft }}\right\rangle$ & Drift field in a drift chamber \\
\hline
\end{tabular}




\section{Chapter 1}

\section{CRIPT: Cosmic Ray Inspection and Passive Tomography}

\subsection{Introduction}

The CRIPT Collaboration is a Canadian scientıfic endeavour to investigate the feasibility of an in-situ real-tıme muon tomography system for use at varıous Canadian sea ports The project is motivated by the increasing need to monitor the contents of imported and exported cargo The number of contamers enterıng Canadian sea ports in 2010 hit 3 million twenty-foot equivalent units ${ }^{1}$ The alm for the technology is to augment and extend exıstıng methods of detecting lllicit high-Z materials such as special nuclear material (SNM) ${ }^{2}$ The other methods include (but are not limited to) sensitıve radıometric detection, cobalt-60 gamma ray ımaging, sniffer dogs, car-born radiation detection, and intelligence gathered by other government departments As of 2004, these methods are applied to $100 \%$ of contamers entering Canada's ports as part of the RADnet ${ }^{3}$ initiative

The operational requirements of the CRIPT muon tomography (MT) system are

\footnotetext{
${ }^{1} 1$ Twenty-foot equivalent (TEU) refers to 20 linear feet of container

${ }^{2}$ Special nuclear material includes uranium, plutonıum, and other radioactive isotopes

${ }^{3}$ RADnet is the Canada Border Service Agency's integrated approach of detecting illicit material through intelligence, passive screening and manual inspections
} 
quite stringent. The false alarm rate must be less than $1 \%$, the detection rate must be greater than $99 \%$, and the scan time is required to be 25 seconds or less. In addition to the detection parameters the MT apparatus must be reasonably robust. It will not only be required to operate in a wide range of temperature and humidity conditions ${ }^{4}$ but also in an environment where heavy machinery and large containers often interact in a violent fashion causing large vibrations in the ground. The MT system will be used to measure how the contents of a container scatter incoming cosmic ray muons and in turn the nature of the contents can be more thoroughly understood.

Muon Tomography is both an attractive and useful technology because it allows for decades of research and development in high energy physics particle detectors to be put to use in a manner which they were not intended but are very well suited. This is no real coincidence due to the fact that the same techniques used in many experiments (particle tracking, calorimetry/spectrometry) can be used to perform tomography given adequate image reconstruction software. Moreover, there is a wide variety of species of detectors that can be utilized. These include:

- Gas filled detectors: Drift Chambers, Multiwire Proportional Chambers, Time Projection Chambers, MicroMegas

- Scintillation detectors: Solid/Liquid/Gaseous configurations, Fibre Optical hybrids $[6]$

The focus of this thesis will be on a type of gas filled detector, namely, the single wire drift chamber. This choice was motivated by several important factors. These factors are: the cost to implement the technology, the position resolution in a tracking: configuration, the lifespan of the detector, and the size of the objects to be scanned. Typical cargo containers are $6.1 \mathrm{~m}$ in length by $2.4 \mathrm{~m}$ in width by $2.6 \mathrm{~m}$ in height which necessitates using a detector that can cover a large area. The CRIPT single wire drift

\footnotetext{
${ }^{4}$ Temperature ranges from $-30^{\circ}$ to $+30^{\circ}$ Celsius typically
} 
chamber is based on a design used for the OPAL barrel muon detector [7] but has been modified to be $2.4 \mathrm{~m}$ long by $1.14 \mathrm{~m}$ wide with smaller prototypes having dimensions of $1.14 \mathrm{~m}$ by $0.46 \mathrm{~m}$. This particular configuration of drift chamber is very well suited for the CRIPT project because it has 2 dimensional read out. That, coupled with a reduced number of readout channels makes it a very cost effective option. Moreover, the ability to utilize a wide variety of drift gases in various electric fields makes this choice highly adaptable. The specifications of the small scale prototypes, along with the specifications of a full scale prototypes, will be explained in detail in a later chapter. A small scale prototype being the actual device used to experiment for the purposes of this thesis.

\subsection{The Collaboration}

The CRIPT group consists of collaborators from across the country. They include, Carleton University, Health Canada (HC), Defence Research and Development Canada (DRDC), Atomic Energy of Canada Limited (AECL), International Safety Research (ISR), Advanced Applied Physics Solutions (AAPS), and the Canada Border Services Agency (CBSA).

Each member is responsible for completing different aspects of the project. Carleton University is utilizing its knowledge in the field of High Energy Physics to contribute a candidate tracking technology along with AAPS. Health Canada is investigating the use of learning algorithms to optimize the tomography process. AECL provides a great deal of simulations. ISR manages the federal account and contributes to simulation work. CBSA provides the guidelines that must be followed in order for the final product to be of any use to the federal government. Lastly, DRDC formally manages the project and contributes to simulation work. 


\subsection{National Security}

In the post $9 / 11$ world, the issue of national security has become increasingly important in the daily workings of most federal governments. The Canadian Federal government, for example, has implemented strategies to mitigate the possibility of security breaches in several ways. The creation of the Canadian Air Transit Security Authority (CATSA), anti-terrorism legislation, and investing public funds for research and development of new technologies are intended to serve this purpose. The CRIPT collaboration is funded by one such investment, namely, the Canadian Research and Technology Initiative (CRTI) grant CRTI-08-0214RD which is a one of many endeavours to source creative new technologies for use in the security field. These advancements are intended to stifle attempts to import or export materials that can potentially be used to make radiological bombs or other kinds of nuclear weaponry.

A closely related topic to national security is nuclear non-proliferation. The 1970 Non-Proliferation Treaty (NPT) ratified by 188 countries in the United Nations (UN) is the most widely accepted piece of international policy in history [8]. That is to say that the notion of non-proliferation is something that many countries with varying political backgrounds can agree on. Though Canada has never had a working arsenal of nuclear weapons, it does have an active nuclear energy program. As a result, nuclear waste must be dealt with in a safe and secure manner. AECL currently stores large quantities of nuclear waste in secure facilities situated near reactors. The contents of these waste containers are not totally certain but nonetheless require ongoing inspection for regulatory reasons. The CRIPT MT apparatus can be used to determine the constituents of this waste in order to ensure it is handled with the proper amount of precaution. 


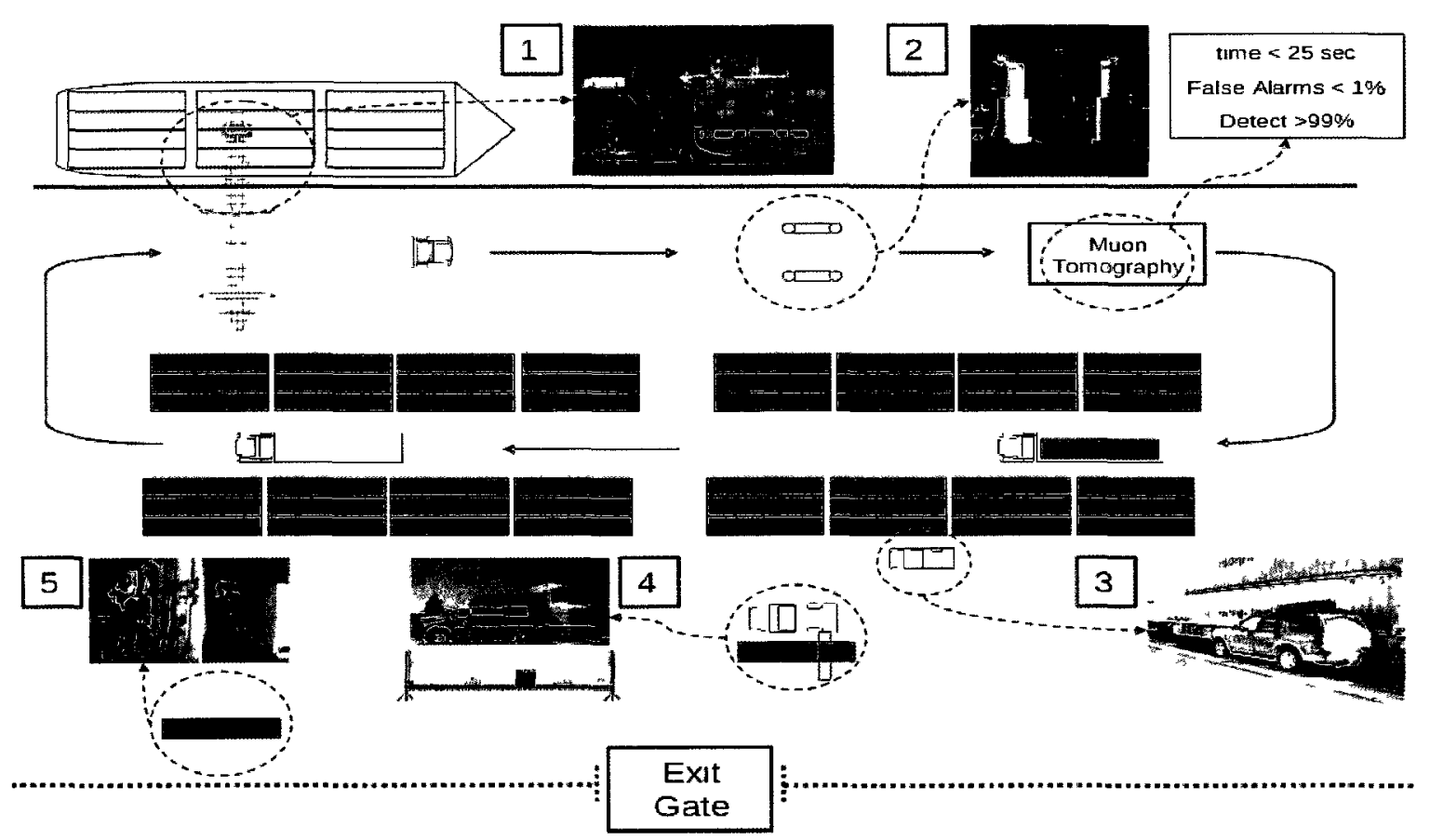

Figure 1.1: Layout of a generic Canadian sea port [1]. Used with permission.

\subsection{Sea Port Operations}

CBSA mandates that the possible implementation of the CRIPT muon tomography system must be of low impact to typical port operations. These operations involve unloading ships containing roughly 6000 containers in approximately 4 to 5 days. This means that one container must be unloaded and processed roughly every minute. This is no small feat, in fact, it is a synchronized act involving the large cranes lifting containers from the ship, placing them on trucks to be moved about the port, and having the containers taken from the trucks and stacked in a highly organized fashion. This process also allows for the containers to be subjected to the screening procedures mentioned in section 1.1 .

The initial scrutiny involves passing containers through $4 \mathrm{~m}$ tall radiation sensitive detectors before they are stacked for storage - step 2 in fig 1.1. Further examination of selected containers takes place after storage and involves car-born radiation detection, 
sniffer dogs, and the Co60 gamma imaging system which can provide a clear image of the contents of the container. If a threat is detected at any one of these stages the container can be isolated for a thorough examination. Should any illicit materials be discovered the appropriate authorities are notified and the contents are dealt with safely.

\subsection{Muon Tomography}

Tomography is an image reconstruction method whereby small sections of a sample are reconstructed individually. The cumulative result of this process can be a complete three dimensional image. The information used to reconstruct images can be sought with penetrating waves, photons, or penetrating high energy particles. In the case of CRIPT the method of probing materials is the use of high energy cosmic ray muons. The physical properties of these particles along with their sources and details in instrumentation will be covered in a subsequent chapter.

The information collected from passive techniques using muons comes through knowledge (measurement) of scattering angles and momentum. Charged particles such as muons can scatter off the nucleus of atoms in the material they are penetrating. This is by Coulomb interaction - it is formally called multiple Coulomb scattering and can account for most of the angular deflection a charged particle experiences over a given length of material. The distribution of angles (for sufficiently small angles) follows a Gaussian distribution. The mathematical details of this physical process will be covered in the next chapter.

In order to apply tomographic imaging to sea containers, the apparatus needs to be sufficiently large to contain one single container at any given time. The proposition is to situate two tracking detectors above, two trackers below, then several (up to 4) layers of detectors below the active volume to measure the muon momentum. This is 


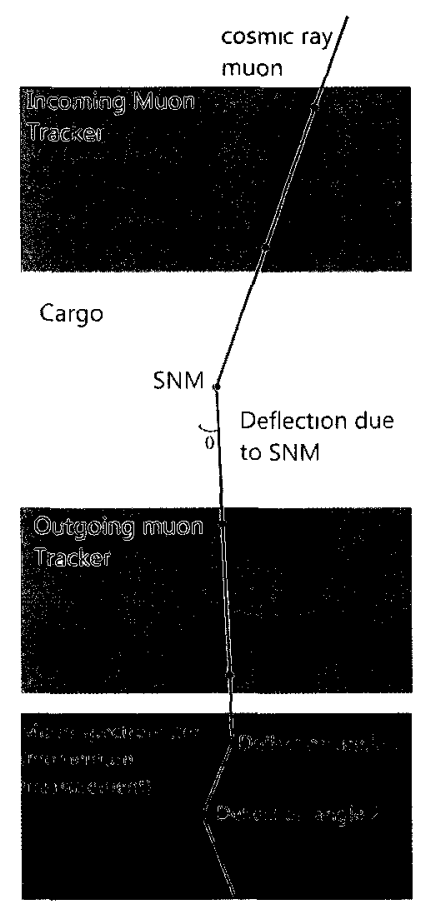

Figure 1.2: Cross section of CRIPT MT system

highlighted by fig 1.2.

\subsection{Review of similar projects}

Muon tomography is an active research field today. There are several groups investigating the prospects of this technology including Los Alamos National Laboratory (LANL) in the United States and a group from the University of Manchester in the United Kingdom.

\section{Los Alamos Project}

This effort has demonstrated [9] that the use of drift tubes is well suited for muon tomography. This groups used an apparatus consisting of 12 individual planes of drift tubes of $0.05 \mathrm{~m}$ in diameter and length $3.65 \mathrm{~m}$ in 3 perpendicular layers both above and below the volume of interest. They demonstrated that with this apparatus it was 
possible to resolve positions to $400 \mu \mathrm{m}$ and angles to $2 \mathrm{mrad}$.

The performance was demonstrated by inserting a $10 \mathrm{~cm}^{3}$ cube of lead into a car engine and letting the apparatus collect cosmic ray muon scattering information. It was shown that a false positive rate of 0 can be obtained with a scan time of 4 min. These results are said to be quite promising.

\section{University of Manchester Project}

This groups is attempting to implement a drift chamber based muon tomography system which, like CRIPT, is basing the detector design on drift chambers used for the OPAL experiment [10]. Their research is still in the early stages but the construction of their prototype detectors has to some extent been completed. They propose an argon-methane gas mixture as the drift gas. The cathode strips for their detectors are more similar to OPAL's than the CRIPT detectors as they use a 'diamond' pattern instead of the CRIPT 'zig-zag'.

\section{Thesis goal}

This thesis will cover, in detail, the necessary physics involved in the kind of instrumentation presented.

The relevant physics includes the possible origins of cosmic rays, the nature of their interactions in the upper atmosphere, a brief discussion of the effects of special relativity, some particulars of the interactions of charged particles with matter, some details on scintillation light, and most importantly the subtleties of electron transport in gaseous media. The instrumentation itself will be described as well due to the specific requirements for the CRIPT drift chambers. The particulars of instrumentation include the use of photomultipliers and a description of the hardware used to extract meaningful data from the drift chambers. The physical constituents of the detectors will be described quantitatively and diagrammatically. 
The two techniques investigated for suitability in collecting drift velocity data will be covered and a conclusion will be drawn as to which is most suitable. The actual results from the measurements of drift velocity for all parameters considered will be presented and analyzed. The limitations of the accuracy of the results will then be discussed. Sources of possible contamination of the drift volume will be explored with detailed simulation as well.

\subsubsection{Author's contribution}

The CRIPT collaboration is a large research effort. This necessitates having particular tasks delegated to those who are particularly well suited to accomplish them. For the construction of one candidate technology the task was given to Carleton University.

The author of this thesis contributed to the effort made by the CRIPT members at Carleton University in the following ways:

- Assembly of the plastic scintillator/light guide portion of the cosmic ray telescope

- The calibration of the photomultiplier tubes used with the light collection system

- A geometrical Monte Carlo to determine the areas of highest illumination of the detector with the cosmic ray telescope

- Geometrical simulation of the timing histograms produced by applying the two methods of data collection

- Leak testing gas manifolds, cleaning cathode surfaces, and checking the cathode boards for the proper resistivity between strips

- Construction of the tower to hold the drift chambers and trigger system 
- Alignment of the small prototype drift chambers in 3 spatial dimensions

- The creation of routines to plot drift velocity data taken from the timing information saved in the existing analysis software

- Simulations using MAGBOLTZ [11]

- Collection and analysis of all raw data used in this thesis 


\section{Chapter 2}

\section{Physics for Muon Detection in Drift}

\section{Chambers}

The focus of this thesis is to build an efficient detector of cosmic ray muons using the transport of ionization in an electric field to measure the hit position ${ }^{1}$. In order to build an effective detector, it is imperative to understand the physics involved from the general elements to the most particular ones. This chapter will cover the origins of cosmic rays, how they interact with Earth's atmosphere, the reason that muons are the main constituent of the cosmic ray flux at sea level, interactions of charged particles ${ }^{2}$ with matter, the measured cosmic ray muon momentum spectrum, multiple scattering, the required instrumentation, and the deposition and transport of ionization in drift chambers.

\subsection{Cosmic Rays}

Given the extremely large size of the known universe there are a plethora of mechanisms able to create cosmic rays. These cosmic rays are some of the most energetic

\footnotetext{
${ }^{1}$ This thesis contributed to the measurement of position perpendicular to the anode wire. The other coordinate is found with a much more involved process

${ }^{2}$ muons
} 
things in the universe itself. To put this in perspective consider that the $\mathrm{LHC}^{3}$ is able to create $14 \mathrm{TeV}$ proton-proton collisions while the most energetic cosmic rays can have energies in the $14 \times 10^{8} \mathrm{TeV}$ regime [12] which is 8 orders of magnitude greater. The composition of cosmic rays impinging on Earth is reasonably well known. Primary cosmic rays consist of particles or nuclei with a lifetime of $10^{6}$ years or more [2] that are created at astrophysical sources, in this case stars, and are the direct results of stellar nucleosynthesis. This includes electrons, protons ${ }^{4}$, helium, carbon, oxygen, etc. Secondary cosmic rays are the results of interactions of primary cosmic rays with interstellar matter ${ }^{5}$. This includes lithium, beryllium, and boron which are not products of stellar nucleosynthesis. The portion of cosmic rays, particularly lower energy ones, that originate from outside our solar system are modulated by our sun's 11-year cycle of varying intensity of solar flares and coronal mass ejections.

The remainder of the cosmic rays, called galactic cosmic rays, are most probably accelerated by supernovae because supernovae result in vast amounts of matter and energy being dispersed into space very quickly. The cosmic rays can traverse the Milky Way several times due to entrapment by galactic magnetic fields [13]. It is possible that cosmic rays may originate from other galaxies as well.

The momentum spectrum of muons at sea level has been found to be dependent upon the angle at which the particular detector is with respect to the vertical. This is shown in fig 2.1 as a summary of results from various research efforts. It should noted that the CRIPT detectors will oriented such that the drift of ionization happens at $90^{\circ}$ to the vertical.

\footnotetext{
${ }^{3}$ Large Hadron Collider

${ }^{4}$ The lifetime of the proton is greater than $2.1 \times 10^{9}$ years which can safely be taken as infinity

${ }^{5}$ Mostly interstellar gas
} 


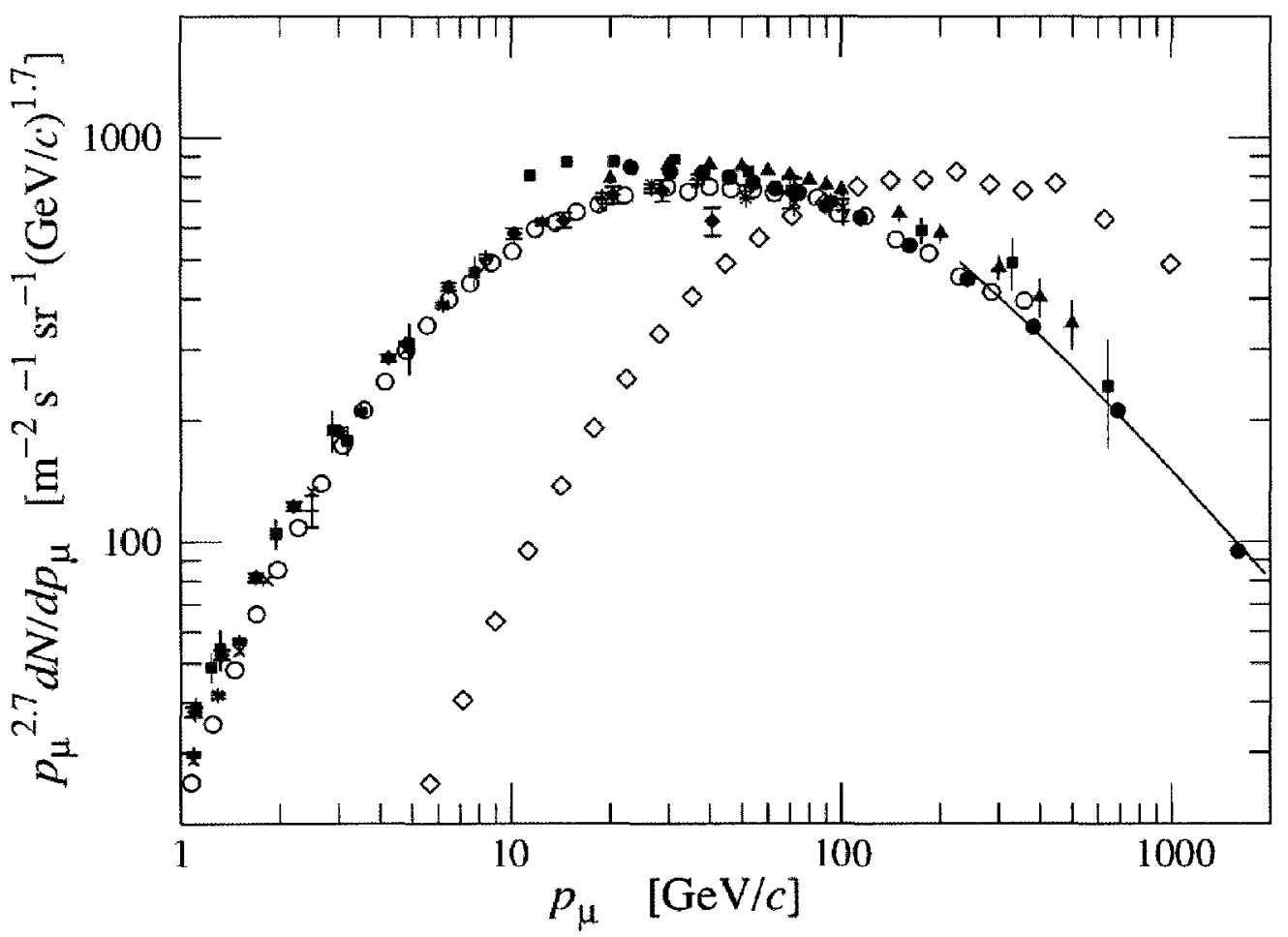

Figure 2.1: Cosmic ray muon spectrum for two angles. The curve with mostly solid data points is for $0^{\circ}$ whereas the second curve (hollow triangles) is for incident muons at an angle of $75^{\circ}$. [2] 


\subsection{Atmospheric interactions}

When a cosmic ray, a proton for example, impinges upon Earth's atmosphere ${ }^{6}$ [14] the probability of interacting with matter becomes quite high. At energies in this regime the strong nuclear force becomes the primary interaction mechanism. That is to say that the quarks in the incident proton interact with the quarks in the atmospheric molecules and create air showers. These showers are typical of the events seen at many high energy collider experiments. There are typically two components and these components depend on the originating particle for the shower. That is to say that hadrons produce mostly hadronic showers whereas electrons/positrons/photons produce only electromagnetic showers.

Electromagnetic showers consist of various processes such as pair production and in turn bremsstrahlung ${ }^{7}$ and continue until the energy of the danghter particles are sufficiently low for them to be absorbed by the medium. Hadronic showers are similar in that they continue via preferred decay channels until daughter particles are of sufficiently low energy. Often the hadrons are themselves charged - thus producing partial electromagnetic showers while simultaneously having hadronic components due to the strong interactions.

The most important difference between the two types of shower is the range over which they occur. Electromagnetic showers are much more easily confined whereas hadronic showers are not. The electromagnetic components are more readily absorbed or scattered by interactions with Earth's atmosphere than their more massive hadronic counterparts. Electromagnetic showers are controlled by electromagnetic interactions whereas the hadronic showers are modulated by the strong nuclear interaction and they scale with something called the nuclear interaction length. The concept of radiation length will be covered in greater detail in a subsequent section.

\footnotetext{
${ }^{6}$ Interactions typically occur at heights of $12 \mathrm{~km}$ above sea level

${ }^{7}$ Radiation produced when a charged particle is decelerated in the case of air showers
} 
For the case of air showers these two quantities are vastly different. Both the radiation length and interaction length of air can be calculated based on tabulated data found in [2]. The radiation length for air is approximately $309.16 \mathrm{~km}$ whereas the nuclear interaction length for air is $747.78 \mathrm{~km}$ which is approximately 2.4 times further.

It has been observed that muons and neutrinos are the most abundant by-products of air showers. Neutrinos interact very weakly which explains the inherent abundance of them - they simply don't interact with an appreciable frequency and continue along their trajectory at nearly the speed of light. Muons on the other hand have a very high penetration power when interacting with matter considering they are heavy for a fundamental particle. This, coupled with special relativity, are the reasons as to why the primary constituent of cosmic ray induced air showers is muons. This process of natural muon production occurs because of the strong interaction between the incident proton and an atmosphere molecule and the weak channel decay producing pions which subsequently decay by the weak interaction to muons. The product pions, denoted by the symbol $\pi$, along with neutrinos and electrons are produced in vast quantities by these cosmic rays. Both charged and neutral pions have very short lifetimes ${ }^{8}$. The preferred decay channels for both these particles are different. Charge must always be conserved so the charged pionic decay must result in charged products. The branching ratio for the process $\pi^{ \pm} \rightarrow \mu^{ \pm}+\nu_{\mu}$ is $99.98 \%$ which explains how muons are produced from primary cosmic rays. The $\pi^{0} \mathrm{~s}$ created in the upper atmosphere decay primarily into pairs of photons which undergo electromagnetic interaction and are more readily dissipated. A highly simplified diagrammatic representation of this can be seen in fig 2.2 .

\footnotetext{
${ }^{8} \pi^{ \pm}$has a mean lifetime of $2.6 \times 10^{-8} \mathrm{~S}$ whereas $\pi^{0}$ is $8.4 \times 10^{-17} \mathrm{~s}$
} 


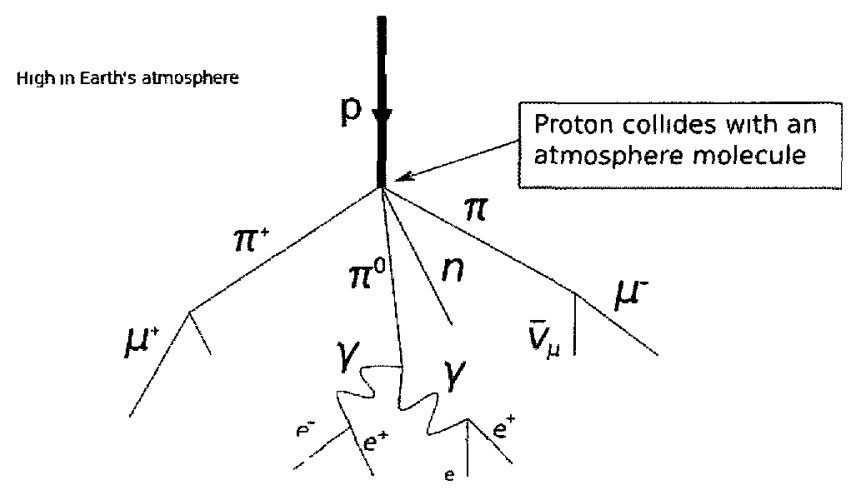

Figure 2.2: Simplified high altitude proton/pion decay. [3]

\subsection{The Muon}

The muon, denoted commonly by $\mu$, is a second generation lepton. It has a mass of 105.7 MeV/ $/ \mathrm{c}^{2}$ and a spin of $\frac{1}{2}$ making it a fermion with a mass over 200 times that of the electron. The muon is considered an unstable particle as its mean lifetime is 2.2 $\mu \mathrm{s}-$ not to be confused with its half-life of $1.4 \mu \mathrm{s}$. This lepton also has a corresponding anti-particle with equal mass and spin but opposite charge called the anti-muon.

The muon was discovered in 1936. This discovery came at a time when the number of particles known to exist was beginning to become cumbersome. This particle zoo was eventually, through the work of many renowned theorists, expressed concisely as the standard model of particle physics. This is summarized by fig 2.3 .

\subsubsection{Cosmic ray muon flux}

Accurate measurements of the cosmic ray muon flux have been made by several groups. One of the most thorough investigations was made by a Japanese group using the $\mathrm{BESS}^{9}$ spectrometer. The experiment was carried out in the late 1990s and underwent several upgrades and the detail of which are discussed in detail in their publication of this research [4]. The experimental apparatus consisted of a high

\footnotetext{
${ }^{9}$ Balloon-borne Experiment with Superconducting Spectrometer
} 


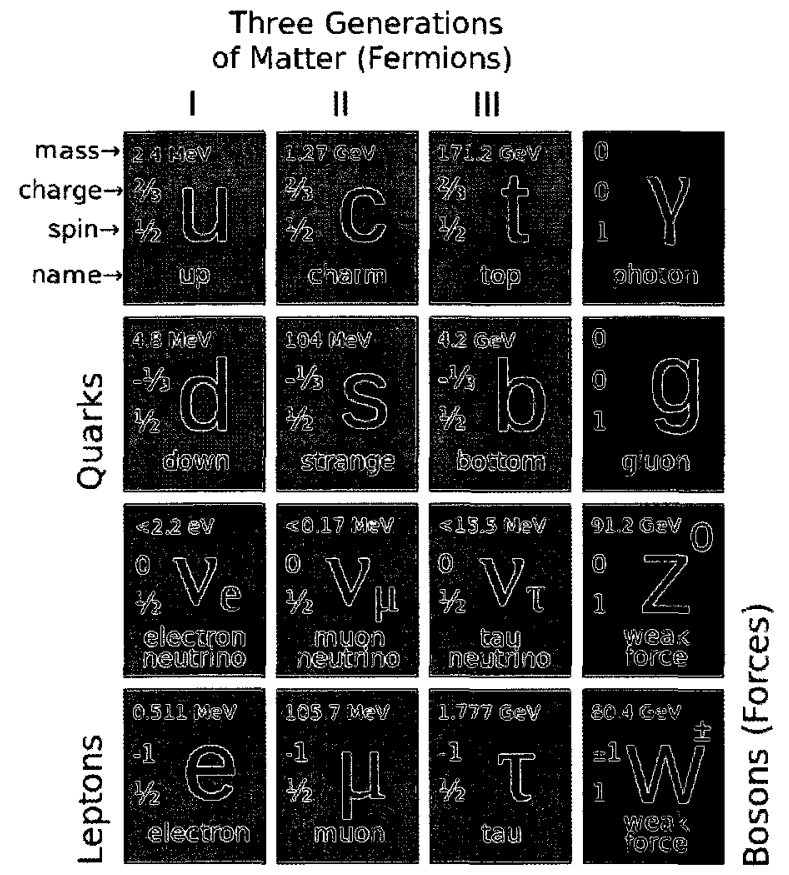

Figure 2.3: The standard model of particle physics. [3]

resolution spectrometer with a large geometrical acceptance in order to study a broad range of cosmic rays. The spectrometer itself consisted of JET-like drift chambers, inner/outer drift chambers, TOF ${ }^{10}$, and acrylic Cherenkov counters. The drift gas used in the drift chambers was a slow gas consisting of argon and carbon dioxide in a $90 \%-10 \%$ mixing ratio.

The experiment collected data at two locations: one in Japan at $30 \mathrm{~m}$ above sea level and another in Canada at $360 \mathrm{~m}$ above sea level. The data presented here were collected in 1995 at the Tsukuba, Japan location. Information about both muons and antimuons were collected independently as well. These data are presented here because they give a reasonable expectation as to the nature of the cosmic ray muon flux that has been detected by the CRIPT prototypes and will subsequently be detected by the final CRIPT MT system.

Fig 2.4 shows the probability as a function of $\mu+$ and $\mu$-momentum. The values of

\footnotetext{
${ }^{10}$ Time of flight detectors
} 


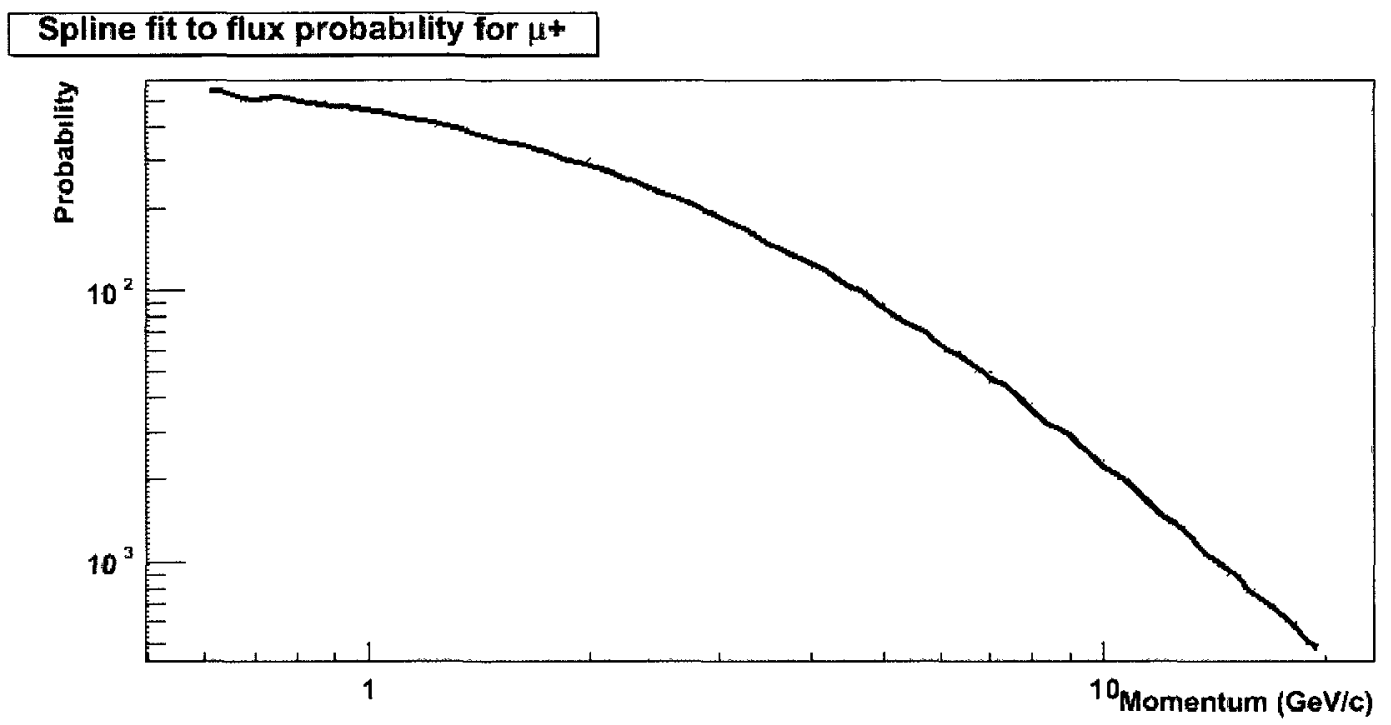

\section{Spline fit to flux probability for $\mu$ -}

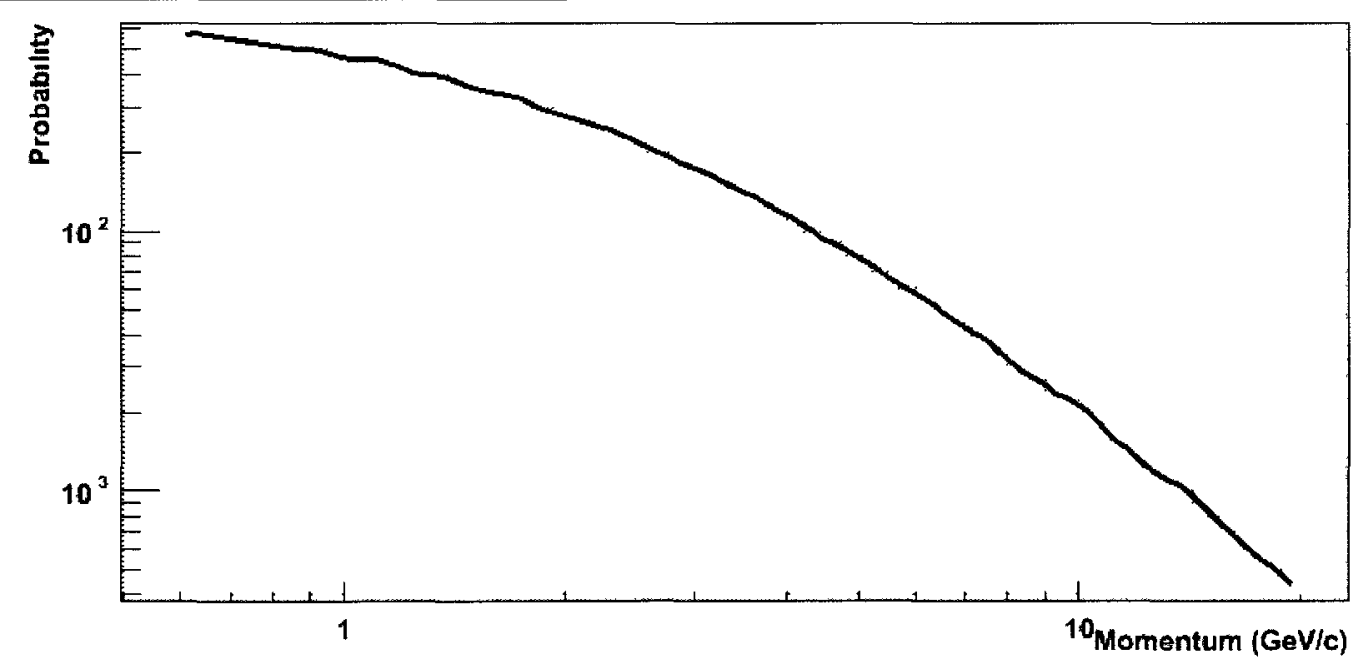

Figure 2.4: Fit to data collected by the BESS experment Note Differential flux Is in units of $m^{-2} s r^{-1} s^{-1}(\mathrm{GeV} / \mathrm{c})^{-1}$ [4] 
the probability are calculated as a ratio of the value of the flux over the total measured flux for the measured momentum spectrum as seen in eqn 2.1. The uncertainty of the momentum comes from the fact that the BESS momentum data are presented in a range. The error is therefore the size of the range with the data point being the mean of that range. The fitting is performed with a cubic spline interpolation routine with ROOT and very effectively estimates the value of the function continuously.

$$
P_{\imath}=\frac{f l u x_{\imath}}{\sum_{\imath=\jmath}^{N} f l u x_{\jmath}}
$$

With this information it is reasonable to expect that the majority of the muon flux detected by the CRIPT drift chambers will be in the minimum ionizing region ${ }^{11}$ or low momentum regime. It is important to note that the CRIPT detectors cannot discriminate between $\mu+$ or $\mu$ - but this is not overly important because the probability curves are almost identical.

The average flux of cosmic rays muons at sea level is 1 muon $\mathrm{cm}^{-2} \mathrm{~min}^{-1} \mathrm{sr}^{-1}$.

\footnotetext{
${ }^{11}$ This will be explained in greater detail in a subsequent section
} 


\subsubsection{Special Relativity}

Given the mean lifetime of the muon, it seems impossible that they survive long enough to reach sea level or even penetrate into the surface of the Earth despite their relativistic velocities. This seeming inconsistency is resolved when special relativity is taken into consideration given that the lifetime is calculated in the rest frame of the muon. If the particle is travelling near the speed of light in a reference frame called $\mathrm{S}^{\prime}$ then an inertial observer in another frame called $\mathrm{S}$ will see the effect of time dilation on the muon's lifetime. The exact amount of time dilation depends explicitly on the speed of the muon as it is produced from pionic decay.

$$
\gamma=\left(1-\beta^{2}\right)^{-\frac{1}{2}}
$$

As an example, let the muon have a speed of $99.99 \%$ of c. The relativistic quantity $\gamma$ can be expressed as eqn 2.2 . This results in $\gamma \approx 71$. Therefore the dilated lifetime of the relativistic muon is $156 \mu \mathrm{s}$. This is given by eqn 2.3 where $\mathrm{t}^{\prime}$ is the dilated lifetime and $\mathrm{t}$ is the rest-frame lifetime.

$$
\Delta t^{\prime}=\gamma \Delta t
$$

If the accelerated muon was travelling in a vacuum it would traverse a distance of $\approx 46 \mathrm{~km}$. Despite travelling through matter (mostly air) these high energy muons are still able to penetrate deep into the Earth's surface. The relativistic quantity gamma, $\gamma$, for a muon in the momentum range of $1-5 \mathrm{GeV}$ would be between 9 and 47 without dimension. 


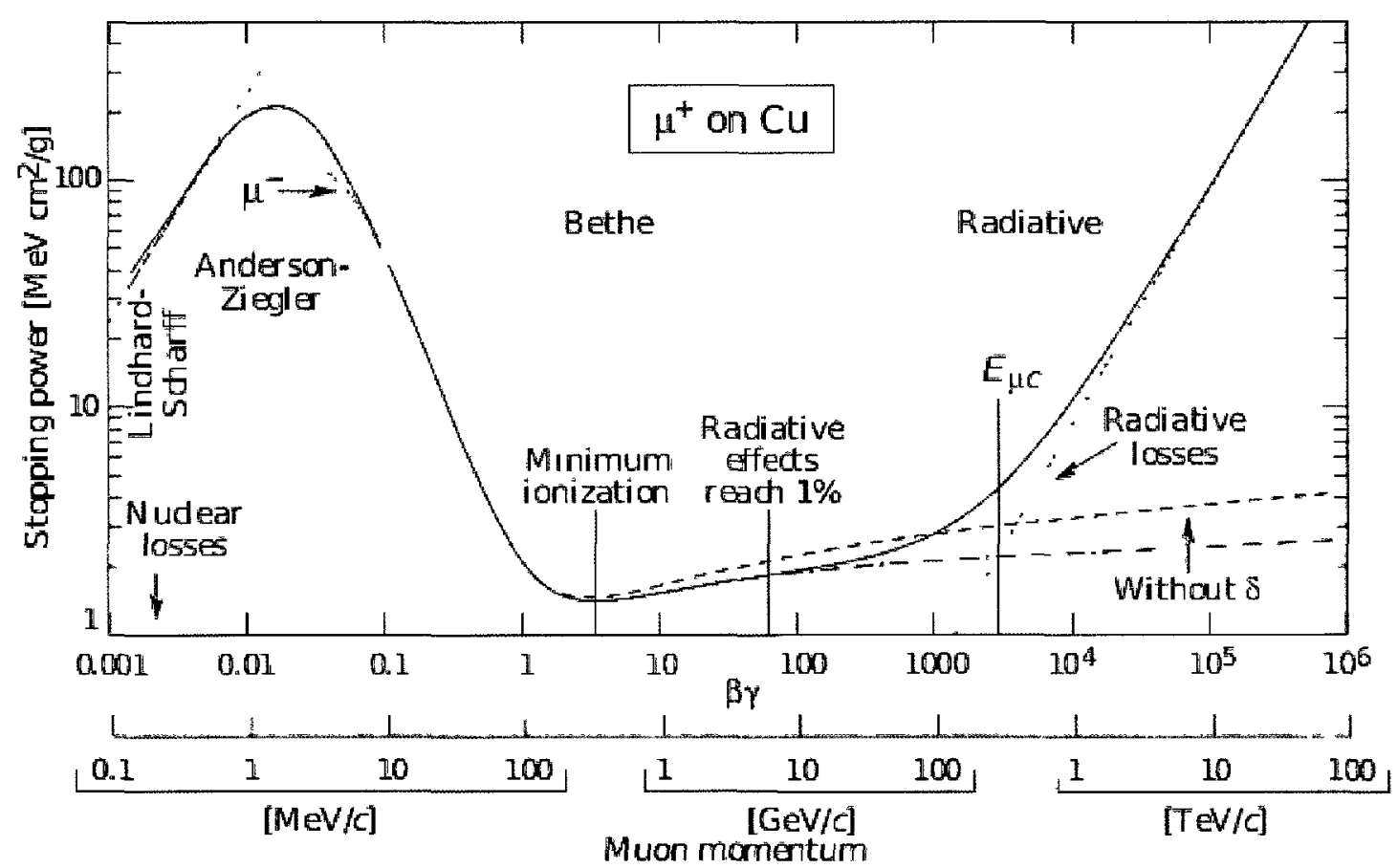

Figure 2.5: Muon interaction with copper [2]

\subsubsection{Interaction with matter}

The discussion of the muon would not be complete without covering its interaction with matter. For a rigorous treatment of this please refer to [2].

The stopping power of a material is described as that material's ability to absorb energy from charged particles as they traverse it. There are four regimes to consider (in order of increasing energy): Lindhard-Scharff, Anderson-Ziegler, Bethe-Bloch, and Radiative. Due to the fact that the mean momentum of the observed muon flux by the CRIPT drift chambers is around $1 \mathrm{GeV} / \mathrm{c}$ (as previously described ${ }^{12}$ ) the Bethe regime will be explained in detail. Moreover, most interactions of particles with matter occur in the regime $0.1<\beta \gamma<1000$ which, as seen in fig 2.5 , is the Bethe-Bloch regime.

The mathematical expression that describes the mean amount of energy lost by

\footnotetext{
${ }^{12}$ In subsection 2.3 .1
} 
charged particles in this region is the Bethe-Bloch equation. It is shown as eqn 2.4.

$$
\left\langle\frac{d E}{d x}\right\rangle=-K z^{2} \frac{Z}{A} \frac{1}{\beta^{2}}\left[\frac{1}{2} \ln \frac{2 m_{e} c^{2} \beta^{2} \gamma^{2} T_{\max }}{I^{2}}-\beta^{2}-\frac{\delta(\beta \gamma)}{2}\right] .
$$

There is an explicit dependence upon the relativistic quantity $\beta$. The minimum around $1-2 \mathrm{GeV}$ stems from the $1 / \beta^{2}$ term in the low energy term which is overtaken by the rising relativistic term due to the medium becoming polarized. The quantities used in eqn 2.4 are shown in table 2.1. The Bethe-Bloch equation remains accurate to within a few $\%$ at describing the mean energy deposited for intermediate-Z materials. The first two terms are simply properties of either the matter through which the muon is passing or the speed and mass of the muon. The third term, $\frac{\delta(\beta \gamma)}{2}$, is a correction for the density effect. This effect is due to the polarization of the medium by highly energetic particles. $\mathrm{T}_{\max }$ is defined as the maximum transferable kinetic energy in a single collision.

$$
T_{\text {max }}=\frac{2 m_{e} c^{2} \beta^{2} \gamma^{2}}{1+2 \gamma \frac{m_{E}}{M}+\left(\frac{m_{e}}{M}\right)^{2}}
$$

This regime is also important because it encompasses a physical phenomena called ionization. In particular, minimum ionization is important when dealing with charged particles interacting with matter. The term minimum ionizing implies that the charged particle deposits a characteristic amount of energy per unit length in the material. The amount is taken as $1.5 \mathrm{MeV}-\mathrm{cm}^{2} / \mathrm{g}$ in [15] and it increases as the velocity of the particle increases which is the intuitive result given the aforementioned dependence on $\beta$.

The rate of energy loss for compounds may also be calculated as required. The fact that the compound can be treated as multiple thin layers of pure elements something called Bragg additivity - means that a simple weighted average ${ }^{13}$ may be

\footnotetext{
${ }^{13} \mathrm{w}_{\jmath}$ is the weighting factor
} 


\begin{tabular}{lll}
\hline \hline Symbol & Definition & Units or Value \\
\hline$M$ & Mass of incident particle & $\mathrm{MeV} / \mathrm{c}^{2}$ \\
$E$ & Energy of incident particle & $\mathrm{MeV}$ \\
$T_{\max }$ & Maximum transferable energy & $\mathrm{MeV}$ \\
$m_{e}$ & Electron mass & $0.510998918(44) \mathrm{MeV}$ \\
$r_{e}$ & Classical electron radius $e^{2} /\left(4 \pi \epsilon_{0} m_{e} c^{2}\right)$ & $2.817940325(28) \mathrm{fm}$ \\
$N_{A}$ & Avogadro's number & $6.0221415(10) \times 10^{23} / \mathrm{mol}$ \\
$z$ & Charge of incident particle & \\
$Z$ & Charge of intermediate material & \\
$A$ & Atomic mass of intermediate material & $\mathrm{g} / \mathrm{mol}$ \\
$K$ & $4 \pi N_{A} r_{e}^{2} m_{e} c^{2}$ & $0.307075 \mathrm{MeV} \cdot \mathrm{cm}^{2} / \mathrm{g}$ \\
$I$ & Mean excitation energy & $\mathrm{eV}$ \\
$\delta(\beta \gamma)$ & Density effect correction & \\
\hline \hline
\end{tabular}

Table 2.1: Variables used in the Bethe-Bloch equation. 
taken as seen in eqn 2.6. This method is not perfect because of the fact that the mean excitation energy $\langle I\rangle$ in this approach is an underestimate because electrons in compounds are more tightly bound than their pure element counterparts.

$$
\frac{d E}{d x}=\left.\sum w_{3} \frac{d E}{d x}\right|_{3}
$$

\subsubsection{Radiation Length}

The radiation length is a relevant property when considering interactions of highenergy electrons in matter [2]. This is necessitated by the electrons' primary energy loss mode of bremsstrahlung. Radiation length, $\mathrm{X}_{0}$, is measured typically in $\mathrm{g} / \mathrm{cm}^{2}$ and it is the mean distance over which a high-energy ${ }^{14}$ electron will lose all but $1 / \mathrm{e}$ of its energy. The radiation length is also a measure of the scale for electromagnetic showers. It is expressed as eqn 2.7 [16].

$$
\frac{1}{X_{0}}=4 \alpha r_{e}^{2} \frac{N_{A}}{A}\left\{Z^{2}\left[L_{r a d}-f(Z)\right]+Z L_{r a d}^{\prime}\right\}
$$

In this context ${ }^{15}, \mathrm{~A}=1 \mathrm{~g} / \mathrm{mol}$, which means the quantity $4 \alpha r_{e}^{2} \frac{N_{A}}{A}$ equals (716.408 $\left.\mathrm{g} \mathrm{cm}^{-2}\right)^{-1}$. Values for $\mathrm{L}_{r a d}$ and $\mathrm{L}_{\text {rad }}^{\prime}$ are given in table 2.2 and can also be found in [2]. The $f(Z)$ function can be expressed, to an accuracy of 4 decimals, up to uranium as eqn 2.8 where $a=\alpha Z$.

$$
f(Z)=a^{2}\left[\left(1+a^{2}\right)^{-1}+0.20206-0.0369 a^{2}+0.0083 a^{4}-0.002 a^{6}\right]
$$

The radiation length is an important factor to consider when dealing with detectors that hope to gain knowledge from charged particles. If the radiation length is small, as is the case for lead ${ }^{16}$, then the material is considered to be a good absorber.

\footnotetext{
${ }^{14}$ 'High energy' in the context of Particle Physics typically refers to energies in excess of $1 \mathrm{MeV}$. ${ }^{15} \alpha$ is the fine structure constant and is equal to $1 / 137.035$ without dimension

${ }^{16} \mathrm{X}_{0}(\mathrm{lead})=6.37 \mathrm{~g} / \mathrm{cm}^{2}$
} 


\begin{tabular}{llll}
\hline \hline Element & $\mathrm{Z}$ & $\mathrm{L}_{\text {rad }}$ & $\mathrm{L}_{\text {rad }}^{\prime}$ \\
\hline $\mathrm{H}$ & 1 & 5.31 & 6.144 \\
$\mathrm{He}$ & 2 & 4.79 & 5.621 \\
$\mathrm{Li}$ & 3 & 4.74 & 5.805 \\
$\mathrm{Be}$ & 4 & 4.71 & 5.924 \\
Others & $>4$ & $\ln \left(184.15 \mathrm{Z}^{-\frac{1}{3}}\right)$ & $\ln \left(1194 \mathrm{Z}^{-\frac{2}{3}}\right)$ \\
\hline \hline
\end{tabular}

Table 2.2: Values for $\mathrm{L}_{r a d}$ and $\mathrm{L}_{r a d}^{\prime}$. All quantities here are dimensionless.

In the case for CRIPT the aim is not to absorb muons at all. Rather, absorption would destroy any possible information that could be extracted from the particles as they are tracked through multiple layers of detectors. That is to say that it is desirable in this case to have a long radiation length. The radiation length for the CRIPT small scale prototype detectors will be calculated once the constituents of the detector and their proportions have been discussed in greater detail.

\subsubsection{Multiple Scattering}

Multiple scattering is a net effect of many interactions of a charged particle as it passes through materials. The charge of the incident particle interacts with the nucleus and is deflected a very minute amount with each interaction. It is the net result of this series of interactions that is observed and it therefore does not, as of yet, have a complete and rigorous treatment on a per interaction basis. Despite this, the net effect of multiple scattering is very well represented by Molière [17]. This model is consistent for small angles but for ones larger than several $\theta_{0}$ is tends to create larger tails to the angular distribution. Reference [2] defines $\theta_{0}=\theta_{\text {plane }}^{r m s}=\frac{1}{\sqrt{2}} \theta_{\text {space }}^{\text {rms }}$ which allows for a Gaussian approximation to the central $98 \%$ of the angular distribution. The width of the angular distribution is given by eqn 2.9 


$$
\theta_{0}=\frac{13.6 \mathrm{MeV}}{\beta c p} z \sqrt{\frac{x}{X_{0}}}\left[1+0.038 \ln \left(\frac{x}{X_{0}}\right)\right]
$$

In this expression the incident particle has charge $z$, velocity $\beta c$. momentum $\mathrm{p}$, and thickness (in radiation lengths) $\frac{x}{X_{0}}$. As in [2], this approximation is accurate to $11 \%$ or better for $10^{-3}<x / X_{0}<100$ for particles with $\beta=1$ for any value of Z. Eqn 2.9 can be used to calculate the multiple scattering by mixtures of various materials as well as single constituent ones. For this case, one cannot simply add values of $\theta_{0}$ in quadrature. This is because the eqn 2.9 is taken from a fit to data by [17]. Instead, it is more appropriate to find the combined radiation length of the compound. This can be done with a simple weighted average if all constituents are known with reasonable certainty.

As previously mentioned, the most effective way of tracking charged particles without interfering with their trajectories is to have a long radiation length type detector. One effect which must also be considered when tracking or calculating the energy resolution of a spectrometer is multiple scattering. Again, CRIPT seeks to use the effect to its advantage. Muon tomography will only work if the tracks through the series of detectors can be reconstructed with a reasonable ${ }^{17}$ amount of accuracy. Multiple scattering depends on the radiation length which has an explicit dependence on the atomic number $Z$. This is why muon tomography is very well suited for detecting high-Z materials.

\subsection{Instrumentation}

The types of instruments used in any experiment are always dependent on the physical processes involved. In spite of this there are usually many ways to achieve a particular goal. Some methods are simply better suited than others. This can be

\footnotetext{
${ }^{17}$ Reasonable accuracy for the CRIPT tracking system is on the order millimetres
} 
highlighted best with a simple example. If one were to perform gamma-ray spectroscopy there are immediately two suitable detectors that can be used. One is a photomultiplier tube and its associated electronics. The other is a germanium based solid state detector. Upon collecting even primitive quantities of data it becomes clear that the energy resolution of the germanium detector is significantly higher than that of the photomultiplier tube ${ }^{18}$. They both work but one will simply work better than the other for spectroscopy. This is not to say that photomultipliers are ancient and of little to no use in contemporary experiments. On the contrary, when coupled with organic scintillating materials they can provide extremely fast responses to detection of charged particles. This is precisely why the CRIPT prototype system used scintillation detectors as the primary event trigger. Scintillation detectors can provide nanosecond time resolution between unique events which is highly desirable for particle tracking. In addition, photomultipliers and scintillators are a much more cost effective solution as germanium based detectors tend to be significantly more expensive.

The particulars of the instrumentation will be covered in the following subsections. A complete discussion of the CRIPT prototype drift chambers and the experimental apparatus will be in a subsequent chapter.

\subsubsection{Scintillation detectors}

The discussion of scintillation detectors, for the purposes of this thesis, will be restricted to organic (plastic) scintillators. The mechanism by which scintillation occurs is fast fluorescence. The incident charged particle ionizes the atoms in the scintillator and excites their electrons to particular excited states. The reason that photo emission is detectable at all is because of the Stokes' shift. This means that the electronic

\footnotetext{
${ }^{18}$ It should be noted that photomultipliers are not technically particle detectors because they do not interact with the primary particles. They collect the produced photons
} 
transitions allow for slightly different wavelengths of light to be emitted than what may have been absorbed. Typically excitation occurs from ground state to the first excited singlet state. The most probable de-excitation is simply from this first state back to the ground state which produces "fast" fluorescence. Another possible transition is from the singlet energy levels to a triplet energy level ${ }^{19}$. This produces what is known as "slow" fluorescence [18]. The difference between fast and slow fluorescence is the decay time between the two processes. The decay time is the characteristic time the electrons remain in the meta-stable excited state before the system relaxes. Relaxation to the ground state occurs from the lowest occupied singlet levels while excitation can reach high vibrational states in the first single level. This results in the Stokes' shift previously mentioned.

Photon yields for organic scintillators are roughly 1 photon for every $100 \mathrm{eV}$ deposited in the plastic [2]. Light produced by plastic scintillators is typically in the ultraviolet and "blue" portion of the visible spectrum. It is important to either choose an appropriate photomultiplier or to use wavelength shifting light guides to correct the wavelength such that a maximum of light is detected. The total detected light by a photomultiplier will depend on the efficiency of the components involved. The light-guide needs to have a very long attenuation length and the photomultiplier a high quantum efficiency.

Consider a $1 \mathrm{~cm}$ thick piece of plastic scintillator. If we assume that only minimum ionizing muons are passing through it, then we can determine that if the density of the scintillator is approximately $1.2 \mathrm{~g} / \mathrm{cm}^{3}$ there will be $1.24 \times 10^{4}$ photons initially produced per incident particle.

\footnotetext{
${ }^{19}$ What is meant by levels here is energy bands
} 


\section{Light guides}

The light guide is used in conjunction with an organic scintillator. The name is very appropriate as they literally help guide the scintillation light from the scintillator to the photomultiplier tube (PMT). Light guides operate on a primary principle of long attenuation lengths. They must also not dissipate the light through transmission as it travels to the PMT. The light guide must also conserve phase space as outlined in [19] because of the Liouville theorem. This means that as light propagates through the light guide the quantity of light per unit solid angle cannot increase once propagation has begun. This implies that reducing the area the light propagates through increased the angular range in order to conserve phase space.

The light guides used for the CRIPT scintillator trigger system consist of multiple strips of plastic moulded in such a way as to mate to both the thin rectangular prism shaped scintillators - as in fig 2.6 - and another light guide with a cylindrical geometry - as in fig 2.7 - which in turn mates with the circular window of the PMT. This is demonstrated with a mock-up shown in fig 2.8 for the sake of clarity. The magnetic shielding has been removed from the photomultiplier tube to show its cylindrical geometry.

The implementation of an effective light guide is relatively straightforward. One observation made through my work with these devices is that a strong (and optically transparent) coupling must be made at each connection. It was found that simply using optical glue to either connect scintillators to light guides or light guides to other light guides was of insufficient mechanical strength. These devices sometimes must be handled and care is unfortunately not always taken. Therefore, a process to increase the mechanical rigidity of the coupled system was used. For the CRIPT trigger system, the scintillators were approximately $3 \mathrm{~mm}$ thick and the light guide (at the scintillator side) was roughly $5 \mathrm{~mm}$ thick. A groove was machined into the 


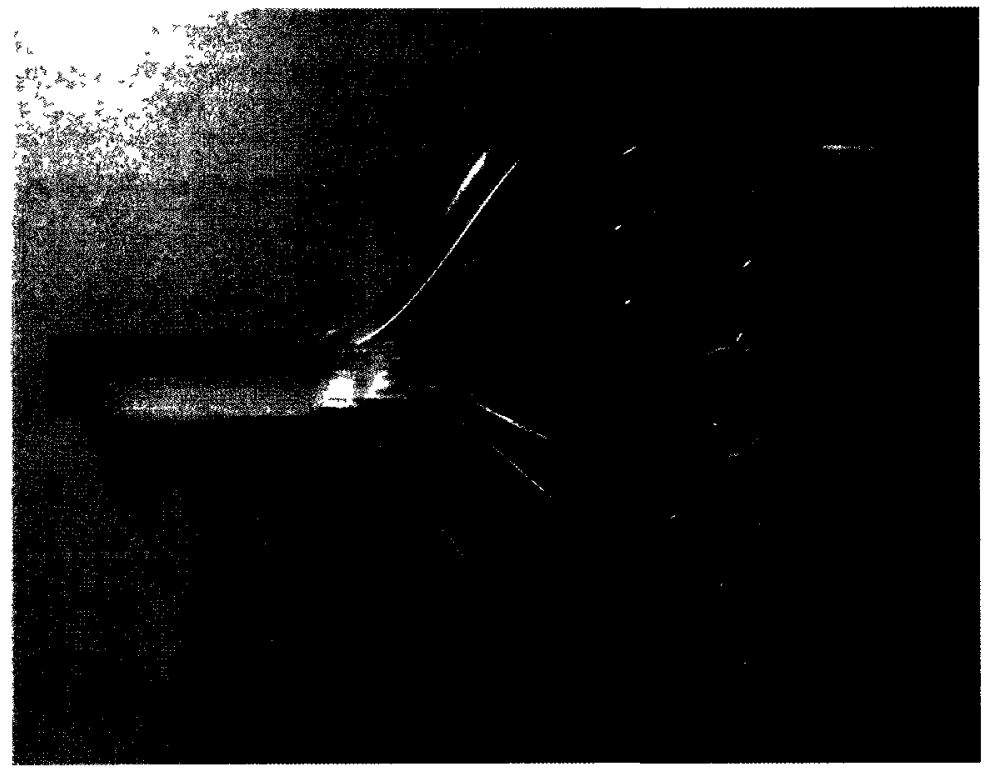

Figure 2.6: Light guide from scintillator side to square face.

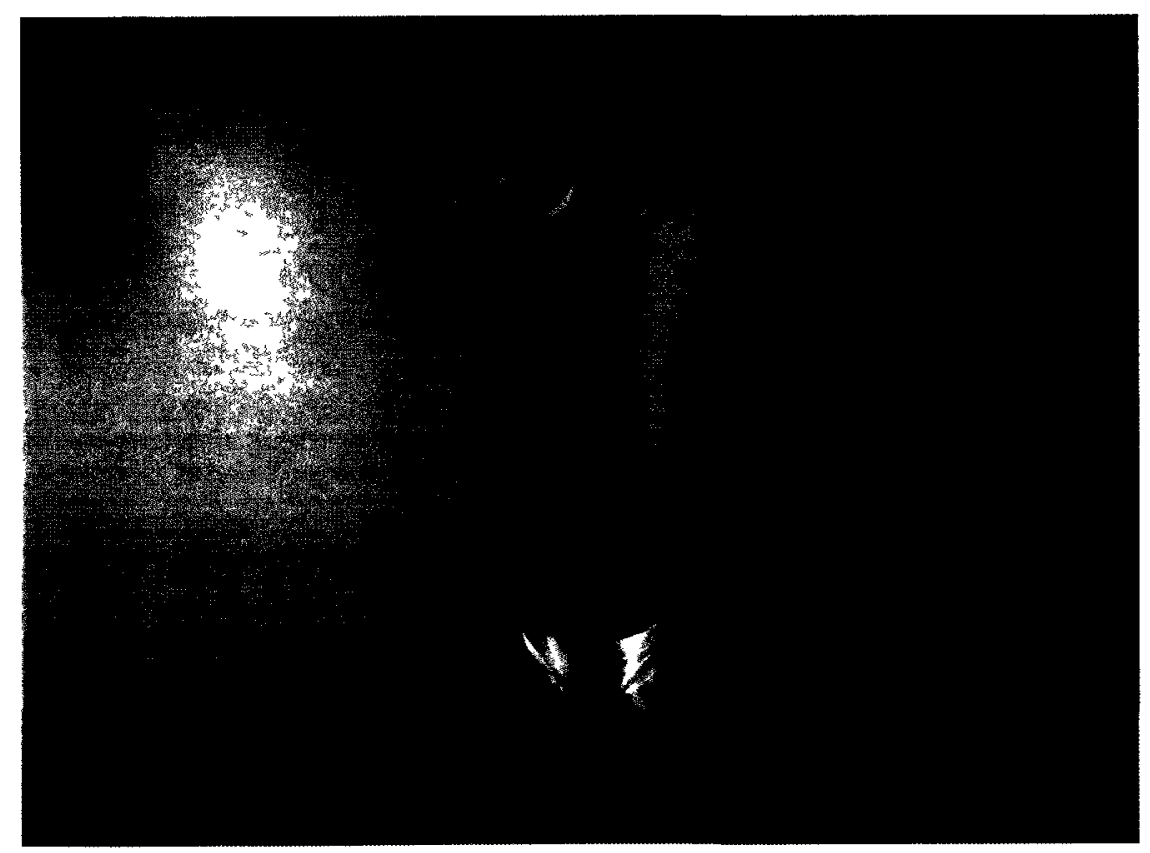

Figure 2.7: Cylindrical light guide 


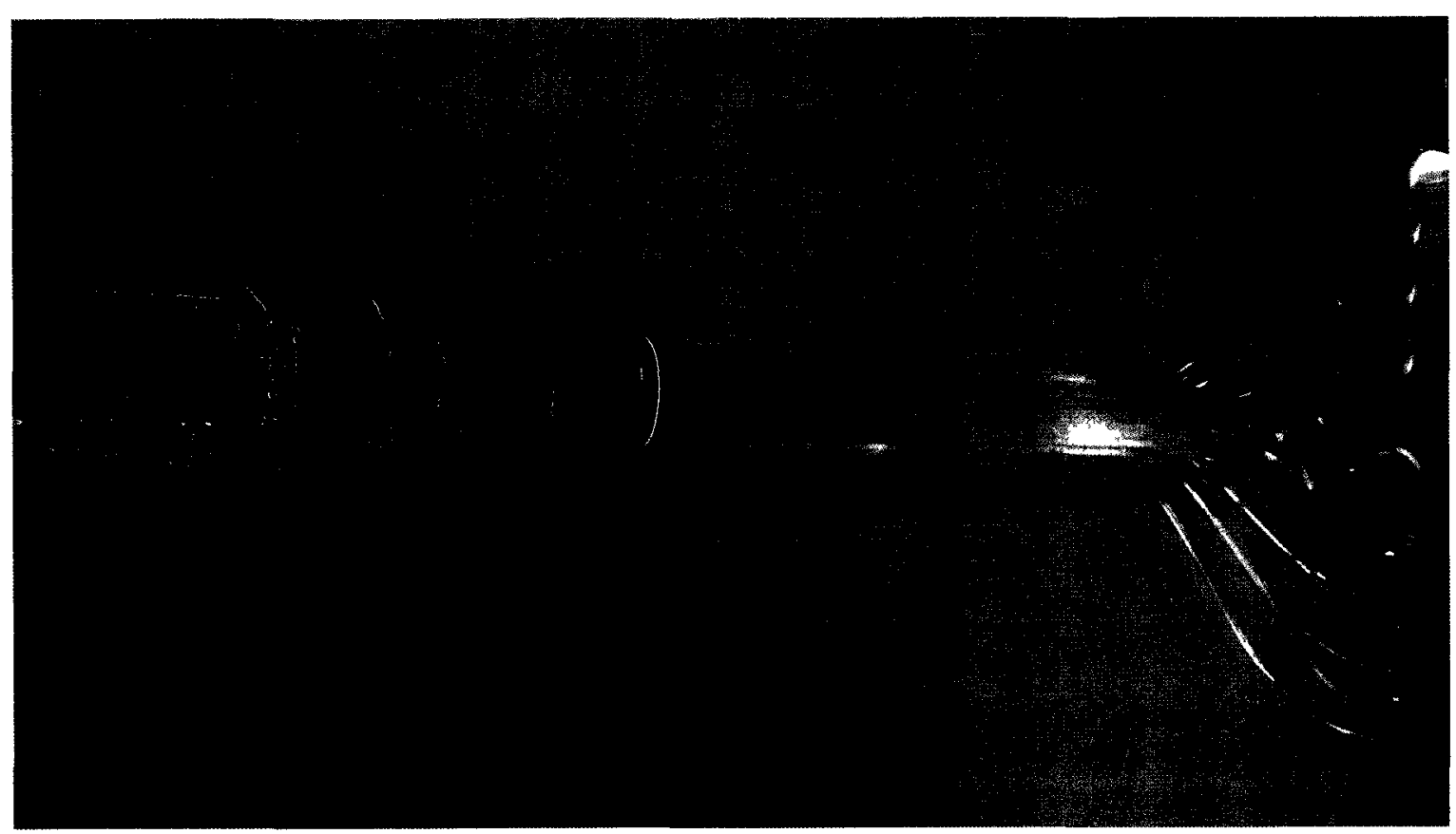

Figure 2.8: Mock-up of light collection system

light guide having an appropriate width to accept the scintillator and an appropriate depth to provide stability. The width is usually increased by $0.1 \mathrm{~mm}$ to allow for fluctuations in the width of the scintillator and the depth was set at $1.2 \mathrm{~mm}$ so as not to reduce the active scintillating area appreciably. Grooves were not necessary when coupling the wide side of the rectangular light guide to the cylindrical light guide because adequate surface area existed for the optical glue to make a strong bond.

A completed and operational scintillator/light guide/PMT system is shown in fig 2.9. Here the magnetic shielding is covering the PMT and the base along with light tight wrapping on all the optical components but it still very much resembles the system shown in fig 2.8 .

\section{Trigger electronics}

The electronics involved in operating the muon trigger system are quite simple for the CRIPT prototyping stage. Involved are discriminators which convert the analog 


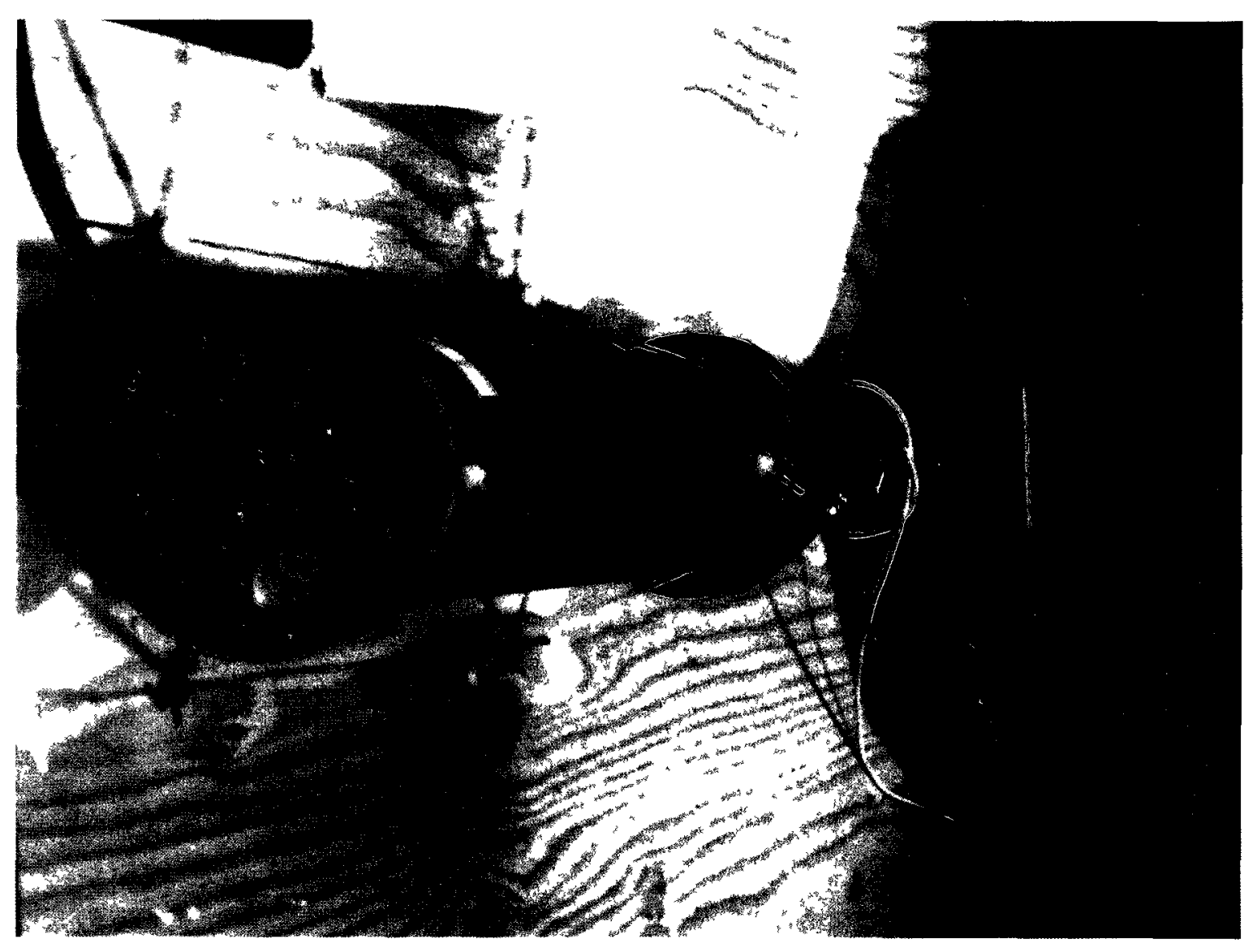

Figure 2.9: Trigger scintillator actually used for this thesis. 


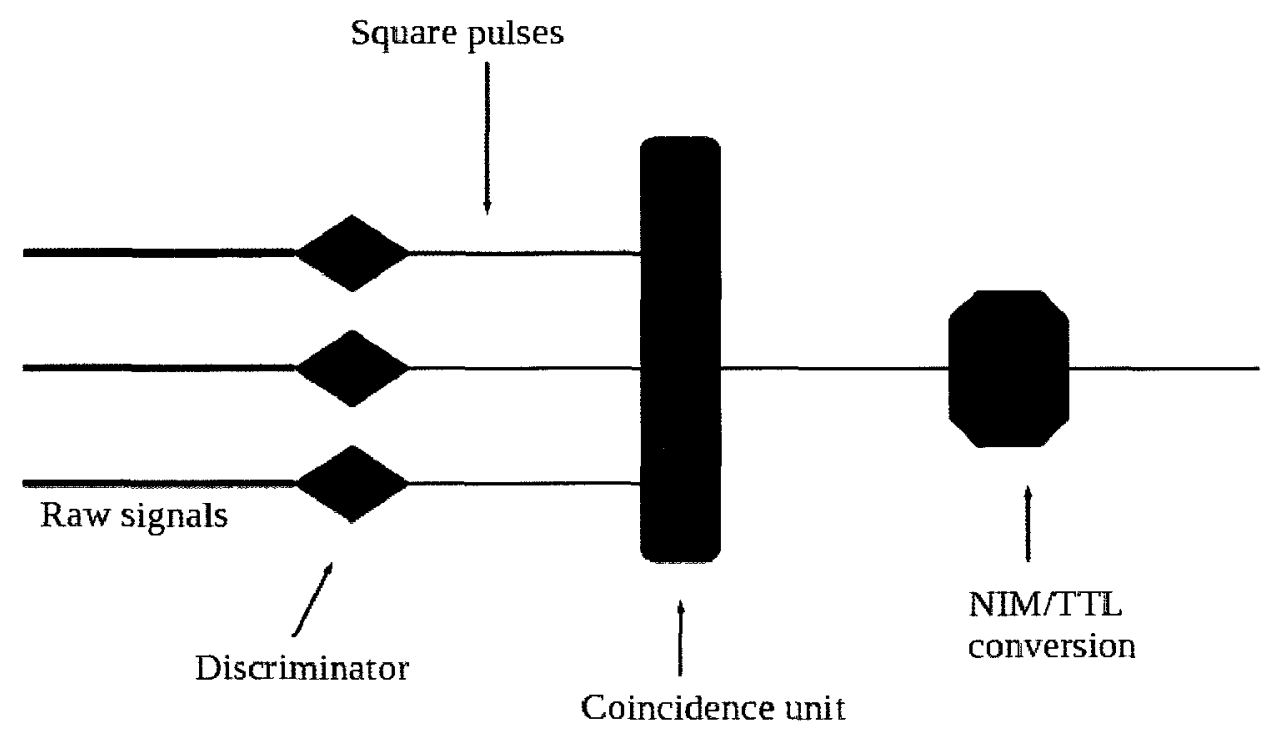

Figure 2.10: Trigger logic circuit

pulses from the photomultipliers to square (digitized) wave forms, coincidence units, a power supply for both logical electronics and PMT's, and a NIM/TTL ${ }^{20,21}$ conversion interface such that the DAQ can read the triple coincidence signals and record data appropriately.

Photomultipliers produce very fast curves which the discriminator promptly converts to a square waveform. This square waveform is then sent to the coincidence unit and subsequently to the NIM/TTL interface.

The discriminator units have variable settings for the threshold of accepted pulses and the width of the product pulses. Ideally the threshold should be set to something slightly higher than the amplitude of the dark current signal from the PMT and the width only several nanoseconds in length. This provides a clean and relatively reliable digital signal from the unit.

The coincidence unit only triggers a positive count when the appropriate number

\footnotetext{
${ }^{20}$ NIM stands for Nuclear Instrumentation Module

${ }^{21} \mathrm{TTL}$ stands for Transistor-Transistor Logic
} 
of digitized inputs essentially occur at the same time. The condition for coincidence is the complete overlap of the leading edge of all input signals [20]. This unit can be set to accept multiple coincidence configurations as well.

Fig 2.10 is a map of the trigger logic circuit. This exact same logical process was used for the experiments presented in this thesis. This circuit can easily be modified to accept more input scintillators if necessary as well. Minor changes to this were in fact made to accommodate another scintillator in order to apply one of the data taking techniques which will be presented in a later chapter.

\section{Photomultiplier tubes (PMT's)}

The photomultiplier can be used to detect the scintillation light propagated from charged particles through the light guides. They do so by the photoelectric effect which means that incident light, given enough energy, can liberate an electron from the photocathode. Typically photocathodes are fabricated with materials with low work functions [21]. Materials typically used in photocathodes are bialkali ones, namely K-Cs-Sb as shown in [21]. Photoelectrons then undergo multiplication. The multiplication happens as the photoelectron is accelerated from dynode to dynode and for every incident photoelectron there are on average approximately 2 photoelectrons produced. The multiplication is given approximately by $N_{\text {ancudent }}^{N_{\text {Dynod }}}$. This leads to the term quantum efficiency which is a measure of how many signal electrons are produced per incident photon. The RCA tubes used in the CRIPT muon trigger system have a quantum efficiency in the neighbourhood of $30 \%$. This is not particularly important though since single photon counting is not the purpose of the PMTs. Rather, it is much more useful to simply calibrate them in such a way as to optimize the signal to noise ratio of the output.

Calibration is very straightforward and easy to manage. When this was completed 
Varying HVI with HV2 8 HV3 constant

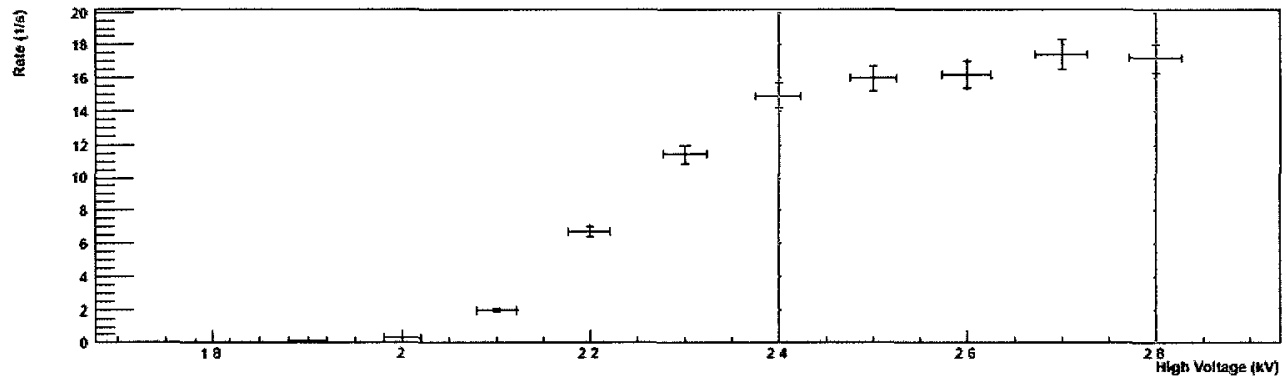

Varying HV2 with HVI 8 HV/3 constant

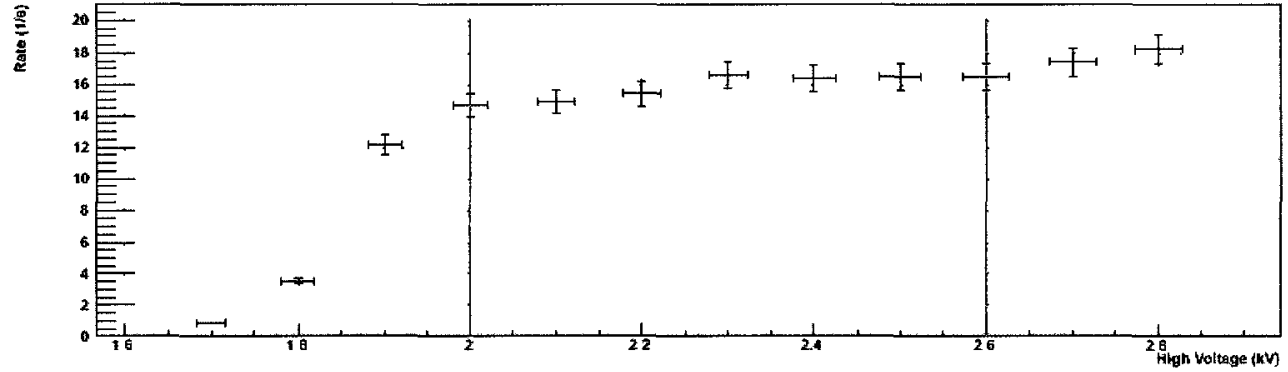

Varying HV3 with RV1 \& HV2 constant

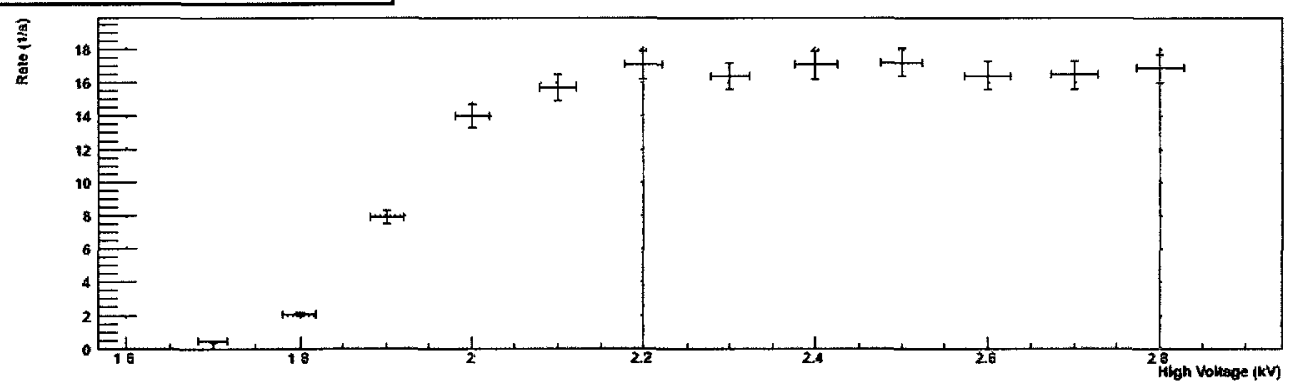

Figure 2.11: Triple rate curves 
for the prototype's trigger system the following method was implemented. Three scintillator/PMT pairs were situated vertically ${ }^{22}$ with the top and middle layers separated by $80 \mathrm{~cm}$ of air. The middle and bottom layers are separated by $1.5 \mathrm{~m}$ of space with $5 \mathrm{~cm}$ of lead to absorb excess short range radiation from either the concrete floor or random gamma rays. The signals from each of the three PMTs are then sent to a discriminator, then to a triple coincidence unit, then finally to an event counter. The lengths of cables between the PMTs and the individual logical circuit components was kept as close to equal as possible in order to mitigate delays in the signals. The high voltage (HV) settings on a single PMT were varied while the others were kept constant and the triple coincidence rate at each $\mathrm{HV}$ value recorded for a given amount of time. Once the plateau in the rate plot was identified, its mean was chosen as the proper HV setting for that PMT. With the top HV layer set, the middle HV settings can be varied and the same process applied. This was of course repeated for the third (bottom) PMT. The spatial locations of the scintillators are not of any real consequence for this process. The flux of cosmic rays seen by the three scintillators simultaneously depends on the angular acceptance. The closer they are together the higher the triple coincidence rate will be.

Fig 2.11 shows the results of one such calibration process. The beauty of this method is that there is a range of high voltage settings which are appropriate choices. For the sake of avoiding damage to the hardware the PMTs are typically set in the mean of the range. The ranges themselves are bounded by the red vertical lines.

\subsection{Ionization}

Ionization is a fundamental phenomenon when using gas filled particle detectors. Moreover, in the case of detectors that use electric fields and sensing wires it is the

\footnotetext{
${ }^{22}$ One on top of the other
} 
crux of their operation. Without the ionization deposited there would be no signal other than noise. The primary mechanism by which muons interact with gas molecules in a gas filled detector is by ionization. The ionization is then localized on the anode wire by drifting it with electric fields. As the electrons are created, positively charged ions are also created in the drift gas. Due to the nature of the electric field, in this case a single wire drift chamber, the positive ions are trapped by the cathode boards.

The interaction between charged particles and gas molecules is completely random. The governing statistics are Poisson statistics where $\lambda$ is mean free path between ionizing interactions (see eqn 2.10), $\mathrm{N}$ is the density of electrons in the gas, and $\sigma_{I}$ is the ionization cross section [22]. The mean number of interactions along a track length is given by $\mathrm{L} / \lambda$. The probability function is given in eqn 2.11 which implies that the probability of having ' $\mathrm{k}$ ' occurences in the interval $\mathrm{L} / \lambda$ is $\mathrm{P}(\mathrm{L} / \lambda, \mathrm{k})$.

$$
\begin{gathered}
\lambda=\frac{1}{N \sigma_{I}} \\
P(L / \lambda, k)=\frac{(L / \lambda)^{k}}{k !} e^{(-L / \lambda)}
\end{gathered}
$$

There exist multiple ways in which electrons can be liberated from a gas molecule. One is called primary and the other secondary. Primary ionization refers to the one or more $\mathrm{e}^{23}$ electrons liberated from the gas molecules by the incident particle. In the context of CRIPT this is the muon. This reaction is as shown in eqn 2.12 where A is an atom.

$$
\mu+A \rightarrow \mu A^{+} e^{-}, \mu A^{++} e^{-} e^{-}, e t c .
$$

The majority of the ionization along the track made by the incident particle is actually secondary ionization [22]. This can happen via interactions with ionization

\footnotetext{
${ }^{23}$ Primary ionization can liberate multiple electrons simultaneously
} 
electrons and gas molecules or by intermediate excited states of the gas. Interactions of the ionization with the gas result in eqn 2.13 as seen in [22]

$$
e^{-}+A \rightarrow e^{-} A^{+} e^{-}, e^{-} A^{++} e^{-} e^{-}, e t c .
$$

Excited metastable states of atoms can also be created through the ionization process. If this metastable state has an energy greater than the ionization potential of the quenching additive ${ }^{24}$ then more ionization may be created.

Often quenching additives are included as a part of drift gases such that the detector may still operate proportionally. Moreover, different mixtures not only have different ionization potentials but they have different transport properties as well. This allows one to essentially customize the operation of a detector depending on the desired result.

As stated in [22] the actual amount of energy deposited by fast charged particles in the gas is quite small. It is so small that it is simply not pragmatic to attempt to measure the initial energy and final energy of the incident particle after ionization has been created when detection of the ionization is the purpose of the main apparatus. As seen in [2] the actual amount of energy deposited by a minimum ionizing particle can vary dramatically depending on factors such at temperature, pressure, and gas mixture.

The range of primary ionization electrons, called the practical range, is parameterized by [22] and is given as eqn 2.14 .

$$
R(E)=A E\left(1-\frac{B}{1+C E}\right)
$$

where $\mathrm{A}=5.37 \times 10^{-4} \mathrm{~g} \mathrm{~cm}^{-2} \mathrm{keV}^{-1}, \mathrm{~B}=0.9815, \mathrm{C}=3.1230 \times 10^{-3} \mathrm{keV}^{-1}$, and $\mathrm{E}$

\footnotetext{
${ }^{24}$ The quencher is the portion of the gas mixture that is not a noble gas. These can include $\mathrm{CH}_{4}$, $\mathrm{C}_{2} \mathrm{H}_{6}, \mathrm{CO}_{2}$, or $\mathrm{C}_{2} \mathrm{H}_{2} \mathrm{C}_{2} \mathrm{H}_{6}$ for example
} 
is the kinetic energy of the primary electron. According to [22] this parameterization is in agreement with electron energies between $300 \mathrm{eV}$ and $20 \mathrm{MeV}$. More than $99 \%$ of primary electrons will have a kinetic energy greater than $10 \mathrm{keV}$ in the first place. This places a higher probability on the primary ionization having a short practical range. A plot pertaining to this can be found in reference [2]. This is for electrons in a $90 \%-10 \%$ mixture of argon and methane ${ }^{25}$. According to [2] the practical range of a $1 \mathrm{keV}$ electron is $70 \mu \mathrm{m}$ which contributes to the fundamental limit of the accuracy of position determination with drift chambers.

The ionization process creates clusters of both primary and secondary electrons along the track of the incident particle. Due to the random nature of this process there exists variation in the number of electrons actually created by a single track. Reference [22] expresses the cluster size distribution as an integral over the spectrum of energy lost, $\mathrm{F}(\mathrm{E})$, for each energy along with the probability of producing, $\mathrm{k}$, ionization electrons, $\mathrm{p}(\mathrm{E}, \mathrm{k})$. This is shown as eqn 2.15. It is still useful to know the average number of electron-ion pairs created by an incident particle along its track. For gases, the average energy lost to create an ion-electron pair is $30 \mathrm{eV}$ [23]. That is to say for a minimum ionizing incident particle, there will be $1.5 \mathrm{keV} / 30 \mathrm{eV}=50$ ion-electron pairs created initially. This is of course a very approximate result.

$$
P(k)=\int F(E) p(E, k) \mathrm{d} x
$$

Insofar as the CRIPT prototype drift chambers are concerned there is no way of determining which electron or cluster from a track was the one actually detected. Rather, the first to arrive at the anode wire is what creates a pulse. Again, this is an artifact of the purely random nature of the creation of ionization by high energy particles.

\footnotetext{
${ }^{25}$ This mixture is commonly known as P10
} 


\subsubsection{Electron transport}

Since the primary ionization has a very short practical range the majority of the transport (drift) of the ionization to the anode is of secondary ionization. The transport of charges through a medium in an electric field causes some change in the ion clusters over time. This is expressed as longitudinal diffusion ${ }^{26}$, transverse diffusion ${ }^{27}$, and drift velocity. These properties depend heavily on the scattering cross-section of the gas mixture itself. As seen in [2] the drift properties of ionization depend on the inelastic cross-section which include the vibrational and rotational modes of the gas molecules. It should be noted that the noble gases have a zero inelastic cross-section below their ionization thresholds. Adding quenchers with large inelastic cross sections to the noble gas results in an effect known as cooling. Cooling simply means the energy of the ionization is reduced to the range known as the Ramsauer-Townsend minimum of argon which occurs at approximately $0.5 \mathrm{eV}$. The Ramsauer minimum is due to quantum mechanical processes in the scattering of the electron with the gas atom where the wavelength of the electron is on the same order of magnitude as the atom [22]. This cooling results in higher drift velocities in these quenched gas mixtures. Another important role of the quenching additive is to absorb ultraviolet photons which may be created by excited noble gas states.

Electron capture is a phenomenon whereby ionization electrons attach to electropositive ions which may by present in the drift gas. The capture cross sections are energy-dependent as seen in [2] and this implies that the probability of capture is a function of the applied electric field inside the drift chamber. Again, this probability is dependent on the particular gas mixture. That is to say some mixtures suffer more heavily from this effect than others. For the CRIPT prototypes it is not desirable to have elements in the gas mixture which increase the capture probability. The presence

\footnotetext{
${ }^{26}$ Parallel to the direction of electron drift

${ }^{27}$ Perpendicular to the direction of electron drift
} 
of such atoms would constitute a contamination of the drift gas and will greatly affect the results of the measurements.

As the ionization approaches the anode wire, the electric field becomes increasingly strong and the electrons can further ionize the gas. This produces an effect called an electron avalanche. This avalanche multiplies the number of ionization electrons in a sort of cascade. The quantitative way of looking at this is something called the gain. Reference [2] expresses this as eqn 2.16 for uniform electric fields. The ratio of $N / N_{0}$ is the gain, $\mathrm{x}$ is the length of distance covered by the avalanche, and $\alpha=1 / \lambda$ is the first Townsend coefficient.

$$
N=N_{0} e^{\alpha x}
$$

In region near the anode wire the electric field is certainly not constant. It varies with distance so eqn 2.16 is not a complete description. Instead, [24] defines the Townsend coefficient, $\alpha_{G}$, to be independent of the initial number of electrons and independent of the derivatives of the electric field and in so doing the gain, $\mathrm{G}$, can be expressed as eqn 2.17 .

$$
G=e^{\int \alpha_{G} \mathrm{~d} x}
$$

Signals produced by ionization in a single wire drift chamber are not actually produced by the electrons themselves. The signal itself is mostly created by positve ions trying to escape this region as the avalanche approaches. The properties of the avalanche depend on the gas mixture and the field strength.

\section{Drift velocity}

The experiments that will be presented in this thesis are intimately involved with the drift velocity of ionization in prototype drift chambers. Therefore a discussion of the 
physics of ion drift will be presented.

The drift of electrons in a medium under the influence of an electric field involves more than a simple macroscopic explanation. Though, the Newtonian expression of velocity eqn 2.18 is incredibly useful in making accurate measurements of the resulting macroscopic behaviour of microscopic interactions.

$$
V=\frac{D}{T}
$$

The source of the microscopic interaction properties for electrons are their very small mass - at least relative to what they are interacting with which is gas molecules. When the electrons scatter off gas molecules, they do so isotropically. Immediately after a collision, the electron will have a velocity, $\mathrm{w}$, and an acquired velocity, $\mathrm{u}$, from the electric field. The acquired velocity is given by the simple Townsend expression eqn 2.19 .

$$
u=\frac{e E}{m_{e}} \tau
$$

This velocity, $\mathrm{u}$, is what is observed as the macroscopic drift velocity. As seen in [22] the additional energy acquired by the electron, on average, is dissipated in the subsequent collision as either recoil or excitation of the collisional atom or molecule. This implies there is a kind of balance of energy acquired and the energy lost in collisions. Reference [22] expresses this as eqn 2.20 where $\mathrm{x}$ is the distance travelled, the number of iterations is $n=(x / u)(1 / \tau)$ which is simply the time of drift divided by the average time between collisional interactions. It is important not to confuse the quantity $\lambda_{E}$ with either wavelength or mean free path as they all share a common Greek character.

$$
\frac{x}{u \tau} \lambda_{E} \epsilon_{E}=e E x
$$


It should be noted that the equilibrium energy, $\epsilon_{E}$, does not consider thermal motion. Reference [22] then expresses the mean time between collisions as a function of the number density, $\mathrm{N}$, the crosssection of collisions, and the instantaneous velocity, $\mathrm{w}$, as eqn 2.21 .

$$
\frac{1}{\tau}=N \sigma w
$$

Another important relationship to consider as outlined by [22] is the one between the instantaneous velocity and the total energy of the drifting electron. This is shown in eqn 2.22 where $\mathrm{k}$ is the Boltzmann constant and $\mathrm{T}$ is the temperature of the gas.

$$
\frac{1}{2} m_{e} w^{2}=\epsilon=\epsilon_{E} \frac{3}{2} k T
$$

In the case for drift chambers the contributions from the applied electric field are far greater than those from thermal motion of gas molecules especially since for the purposes of this thesis the detectors are operated at normal temperature and pressure. Therefore, the thermal contribution can simply be neglected. Finally, if we combine eqn 2.19 , eqn 2.20 , and eqn 2.22 one obtains the following two expressions as stated by [22]:

$$
\begin{aligned}
& u^{2}=\frac{e E}{m_{E} N \sigma} \sqrt{\frac{\lambda}{2}} \\
& w^{2}=\frac{e E}{m_{E} N \sigma} \sqrt{\frac{2}{\lambda}}
\end{aligned}
$$

There exist powerful simulation tools, which are used in the research presented here, that allow one to obtain curves for both types of diffusion and drift velocity as a function of electric field for many different gas mixtures. MAGBOLTZ was used to obtain such curves for the purposes of predicting the behaviour of the CRIPT 
prototypes and to also understand the subsequent results. 


\section{Chapter 3}

\section{CRIPT prototypes}

The purpose of the prototyping stage is to determine first the suitability of a particular design. A second, and relevant for the CRIPT collaboration, purpose is to generally determine the suitability of drift chambers to meet the goals of implementing a muon tomography system. The focus of this thesis will be on an aspect of the primary purpose of prototyping. Namely, measurements were made, not for the purpose of meeting a requirement of the collaboration per se but simply to characterize how the prototype performs under different conditions.

\subsection{Small-scale prototypes}

The CRIPT small scale drift chambers were built in part with the help of Communications Research Centre (CRC) - Shirley's Bay and the National Research Council (NRC). The portion of the fabrication process that required outsourcing was the machining of the cathode boards. The CRIPT prototypes, as mentioned earlier are based on the drift chamber design used in the OPAL muon detectors. They are similar in that the cathode boards are both a $60 \mu \mathrm{m}$ layer of copper adhered to an FR4G10 backboard of thickness $3.1 \mathrm{~mm}$. There are two cathode boards per detector separated by a distance of $1.5 \mathrm{~cm}$ of space. This space is where the drift gas flows between input 


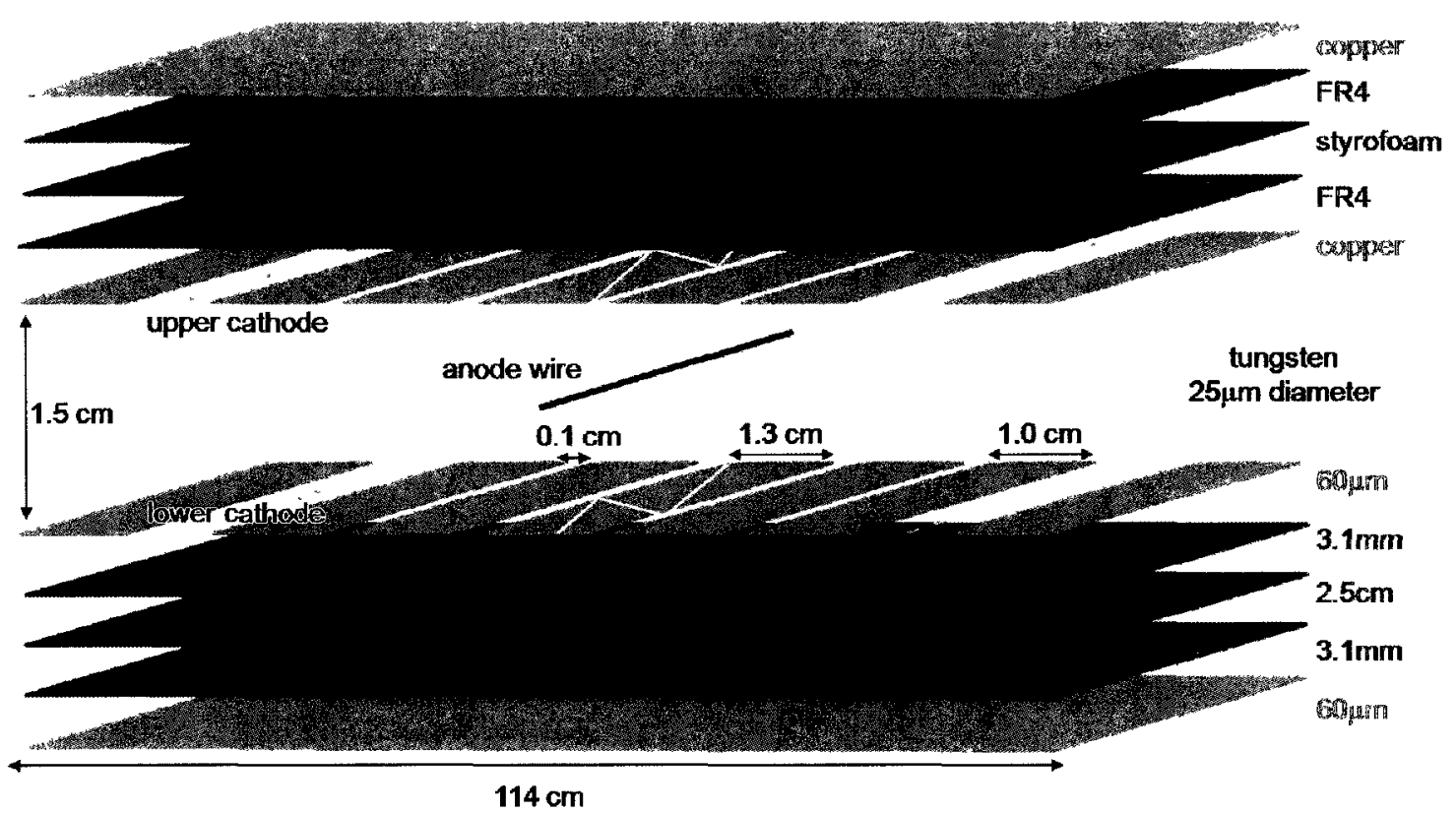

Figure 3.1: Exploded view of CRIPT prototype. Constituents and their thicknesses are listed along with 2 spatial dimensions. The gas flows in the $1.5 \mathrm{~cm}$ tall region between the two cathode planes. The quantities on the right denote the thickness of layers they pertain to. The correlation between material and quantity is by colour code.

and output gas manifolds and also where the $25 \mu \mathrm{m}$ tungsten anode wire is situated. The anode wire is held in place by no more than $2 \mathrm{~N}$ of force and is situated an equal height from both cathode boards, and from both gas manifolds and both aluminum side rails. That is so say it is sitting in the geometrical center of the chamber. The tension applied to the wire depends on the physical properties of the wire itself such as material, Young's modulus, etc. The other components of the drift chamber are, as mentioned, G10 gas manifolds, aluminum side rails, and polystyrene. The orientation of these components can be seen in fig 3.1. It is also important to note that the drift chamber is operated at normal temperature and pressure. ${ }^{1}$

In fig 3.1 the length dimension parallel to the direction of the anode wire is 46 $\mathrm{cm}$ for the small scale prototypes. The total length perpendicular to the wire is 1.14

\footnotetext{
${ }^{1} \mathrm{NTP}:$ Temperature $=20^{\circ} \mathrm{C}$ Pressure $=1 \mathrm{~atm}$
} 
$\mathrm{m}$. The height of $1.5 \mathrm{~cm}$ yields a drift volume of $7866 \mathrm{~cm}^{3}$. It should be noted that the drift volume is effectively bisected by the anode wire. This means the maximum drift distance ionization can travel is $57 \mathrm{~cm}$. This symmetry will be exploited when the particulars of the experiment presented in this thesis are explained.

The CRIPT prototypes have one distinguishing feature, other than particulars of dimension, that make them novel. That is the machining of a 'zig-zag' pattern on the upper and lower cathode boards. The upper and lower boards' 'zig-zag' are offset by $1 / 4$ of a wavelength of the pattern and the wavelength of the pattern is $40 \mathrm{~cm}$. The central electrode - the one with the 'zig-zag' pattern is $1.3 \mathrm{~cm}$ wide whereas all other electrodes are $1 \mathrm{~cm}$ in width with a gap of exposed FR4G10 of $0.1 \mathrm{~cm}$. This is shown in fig 3.2 .

The radiation length was calculated, as shown in subsection 2.3.4, and yielded a value of $12.74 \mathrm{~m}$. This value is more than acceptable given that the total thickness from exterior copper layers is less than $13 \mathrm{~cm}$. This simply means the detectors will not attenuate the spectrum of muons they are detecting.

\section{Large scale prototype}

A larger scale CRIPT prototype drift chamber was also constructed along with the 3 small scale prototypes. The two types are identical in the direction perpendicular to the anode wire. That is to say they have the exact same electrode size, number, and cathode 'zig-zag' and the same maximum drift length. The difference lies in the large prototype being elongated to a length of $2.14 \mathrm{~m}$ in the direction of the anode wire. The thickness of the detector was also increased as it was found that the larger area caused some deformation in the center of the detector. The only component that can be increased in thickness is the polystyrene because it has the lowest effect on the radiation length of the detector. The deflection is only acceptable up to a 


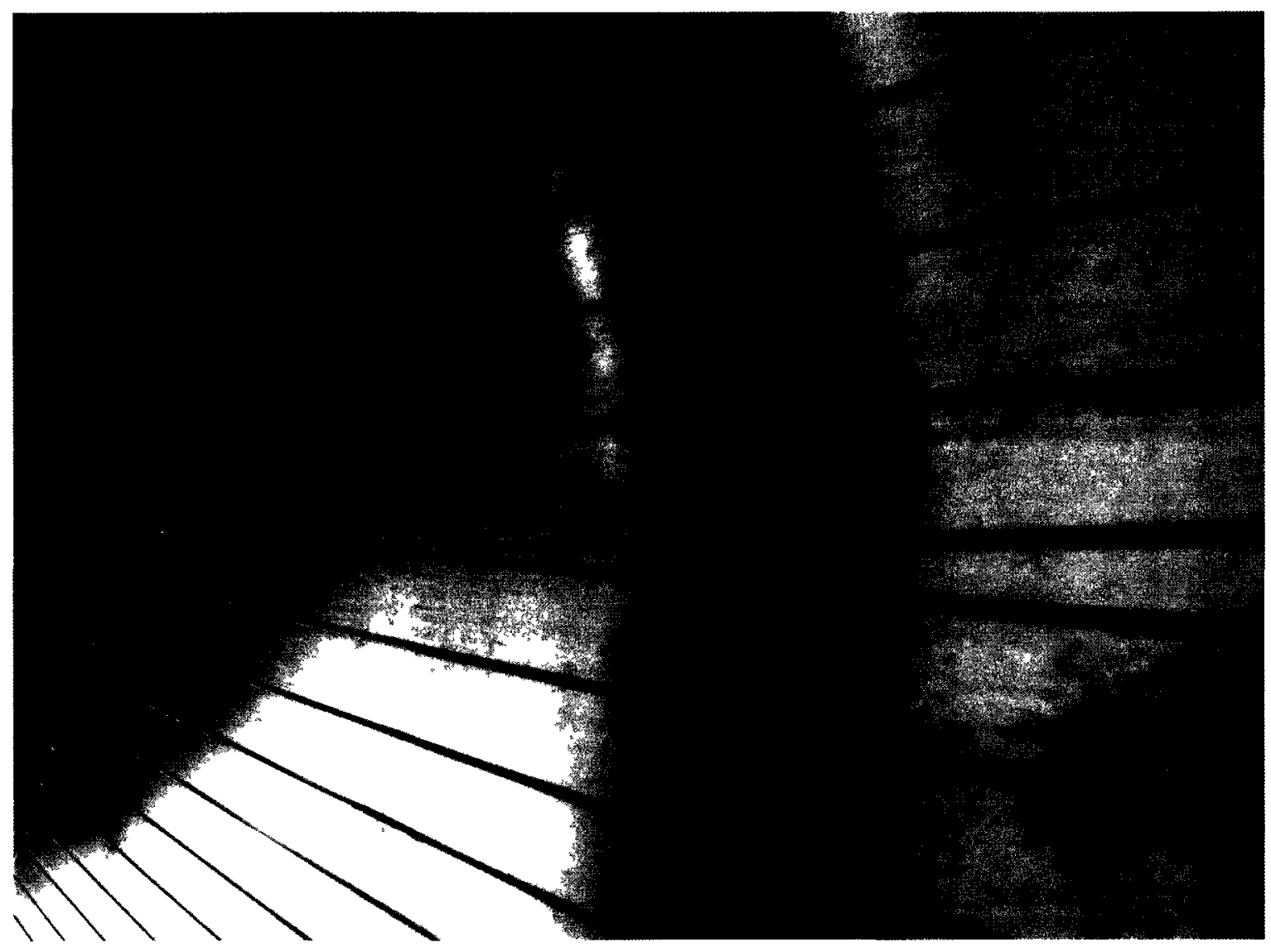

Figure 3.2: Close-up of CRIPT zig-zag cathode board. 


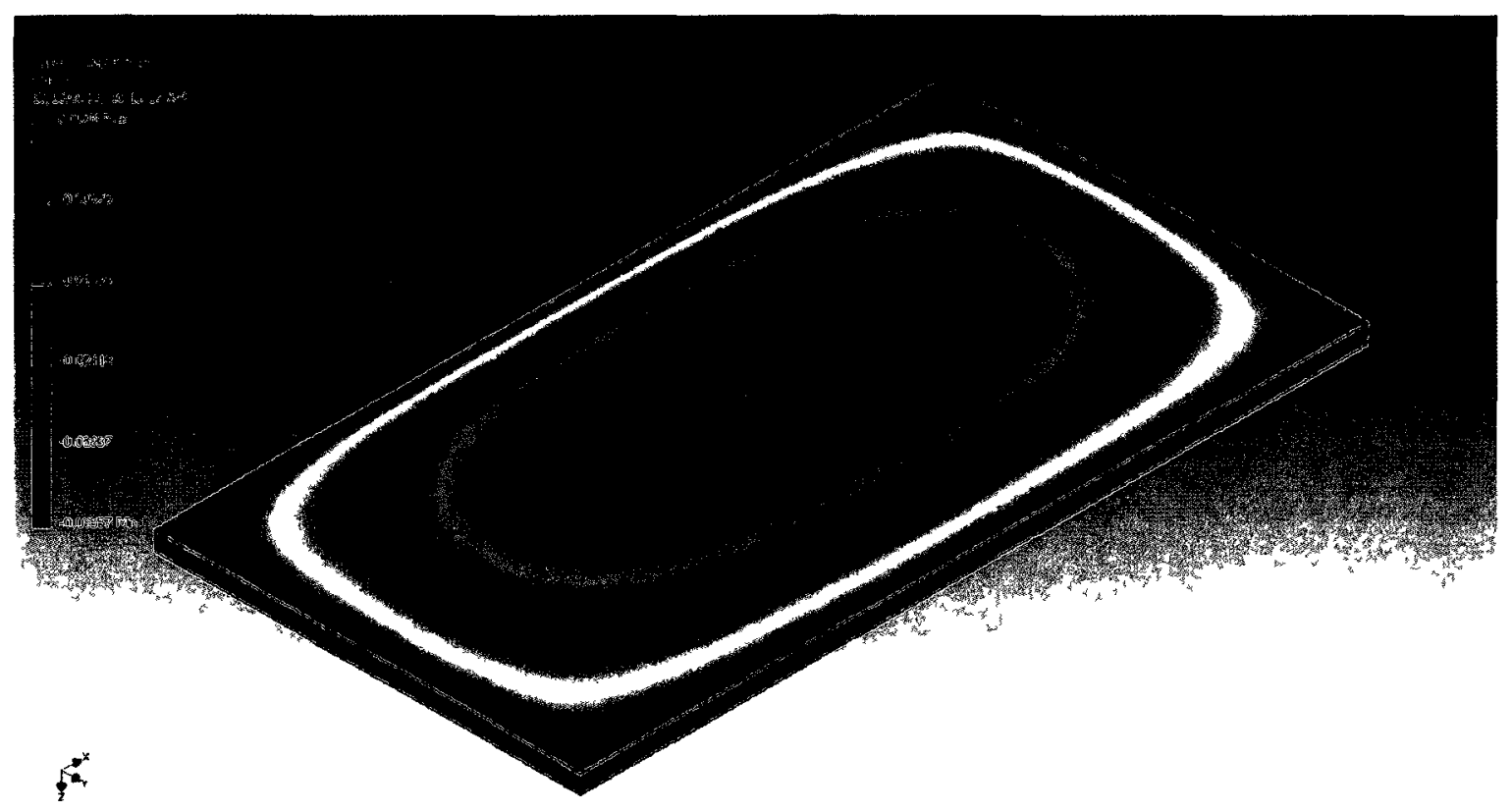

Figure 3.3: Engineering drawing of the deflection experienced by a large scale drift chamber lid at atmospheric pressure. [5]

particular tolerance before it becomes drastic enough that it produces serious irregularities in the electric field inside the detector. The amount of deflection estimated to occur with a thickness of 2 inches $^{2}$ is shown in fig 3.3 and amounts to $0.9 \mathrm{~mm}$ at atmospheric pressure. Another slight modification that was required to fabricate this larger detector was a support system for the anode wire. Regardless of proper tensioning initially the wire will sag over the total length of $2.14 \mathrm{~m}$. This problem was solved by stringing a nylon string across the detector perpendicular to the wire and making contact with the wire at its midpoint. This provided the necessary support without electrostatically interfering with the operation of the detector.

\subsubsection{High voltage (HV) electronics}

For the small scale prototype used in this thesis a CAEN N470 HV power supply was used to power the anode wire and the cathode strips. A simplified circuit diagram

\footnotetext{
${ }^{2} 2$ inches $=5.08 \mathrm{~cm}$
} 


\section{Right hand side board (RHS)}

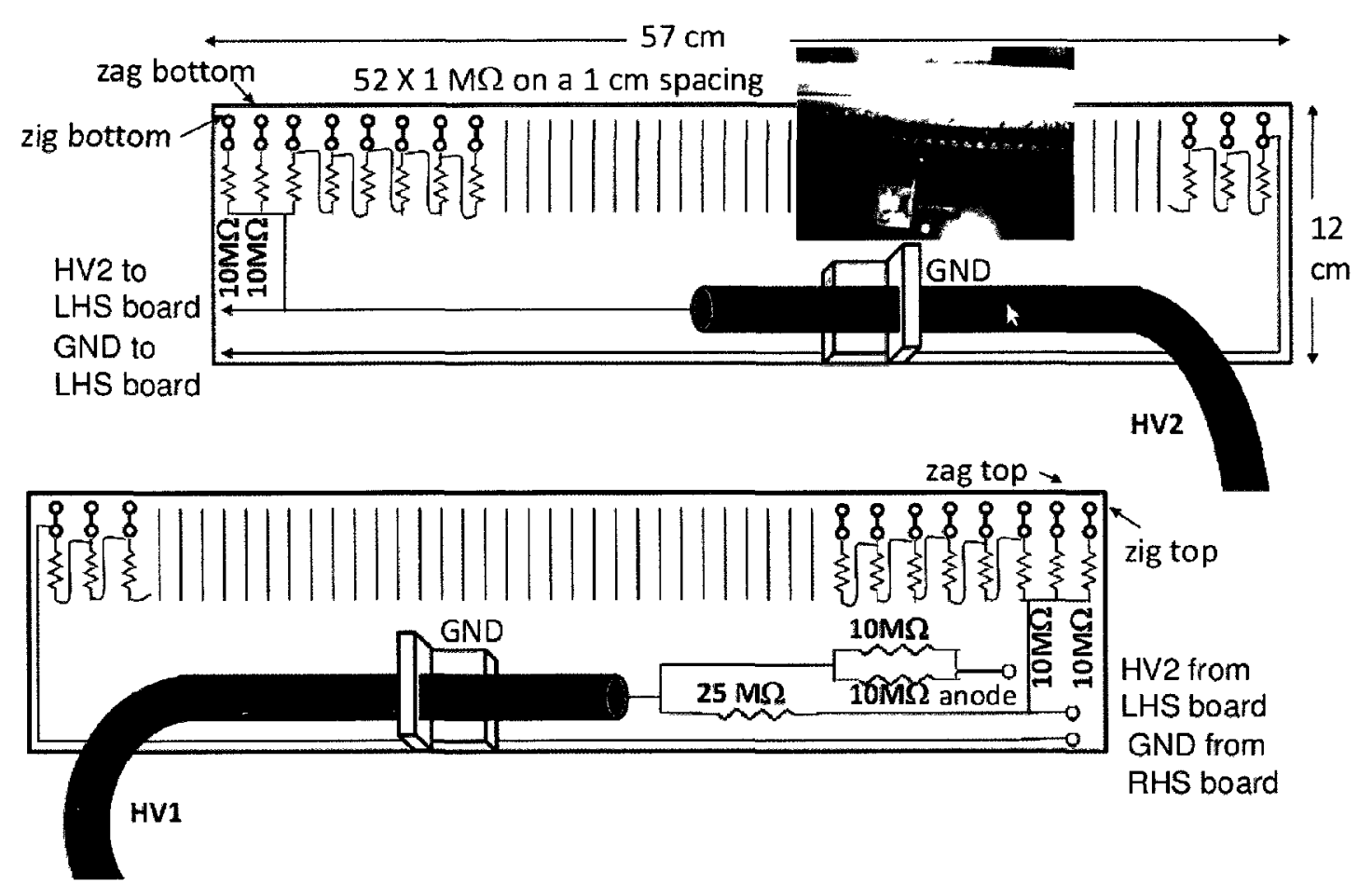

\section{Left hand side board (LHS)}

Figure 3.4: Simplified high voltage board diagram

of the HV boards is shown in fig 3.4. The components labelled 'HV ladder' are the chain of $1 \mathrm{M} \Omega$ resisters that supply direct current to the individual electrode strips. Both the top and bottom portions of the 'zig-zag' strips are separated from the power supply by a $10 \mathrm{M} \Omega$ resister each. The part with the capacitor then heading to ground is the anode wire where the detector itself acts as the capacitor. The components labelled 'HV 1' and 'HV 2' are the high voltage connections from the power supply. 'HV 1' supplies the potential for the anode wire while ' $\mathrm{HV} 2$ ' supplies the two $\mathrm{HV}$ ladders. This high voltage supply circuit is again based on the OPAL barrel muon chamber high voltage boards. This can be seen as it was used in fig 3.5.

The HV boards, and the properties of the anode wire, effectively limit the voltage 
that can be applied to the drift chamber before some kind of breakdown occurs. Types of breakdown include the circuit boards themselves arcing and depositing carbon in the wafer which essentially destroys them over time. The anode wire can potentially break which would render the detector useless. The wire can be replaced but it requires all the individual $\mathrm{HV}$ connections for each cathode strip to be cut then resoldered once the repair is completed, the seal on the chamber breached, and a new wire strung. That is to say that care must be taken when using these detectors at high voltages near their operational limits. The boards were tested up to $10 \mathrm{kV}$ before any kind of strange behaviour was noticed. The strange behaviour that occurred was a saturation of the preamplifier board. This saturation over powered the safety diodes in place and temporarily rendered the preamplifiers themselves useless. This damage seemed to disappear if current was shut off from the detector for a short period of time however.

It is necessary to understand the operation of the $\mathrm{HV}$ component of the detector because it is how one sets the appropriate drift field in the active volume. Since there are two high voltage boards and the cathode planes are symmetric about the wire the value of the drift field ${ }^{3}$ is given by eqn 3.1 .

$$
\left\langle E_{d r_{\imath} f t}\right\rangle=\frac{V_{\text {cathode }}}{D_{d r \imath f t}}
$$

In eqn $3.1 \mathrm{~V}_{\text {cathode }}$ is the voltage applied from the $\mathrm{HV}$ power supply to the $\mathrm{HV}$ ladder ${ }^{4}$. This is also the potential across the central electrode. For the experiments in this thesis values of $3 \mathrm{kV}, 4 \mathrm{kV}$, and $6 \mathrm{kV}$ were examined. $\mathrm{D}_{\text {drift }}$ is the maximum drift distance ionization can traverse in the chamber which for the CRIPT prototypes is $57 \mathrm{~cm}$.

A useful method for diagnosing potential problems with the detector is to observe

\footnotetext{
${ }^{3}$ At distances outside of the gas gain region which are close to the wire

${ }^{4} \mathrm{HV} 2$ in fig 3.4
} 


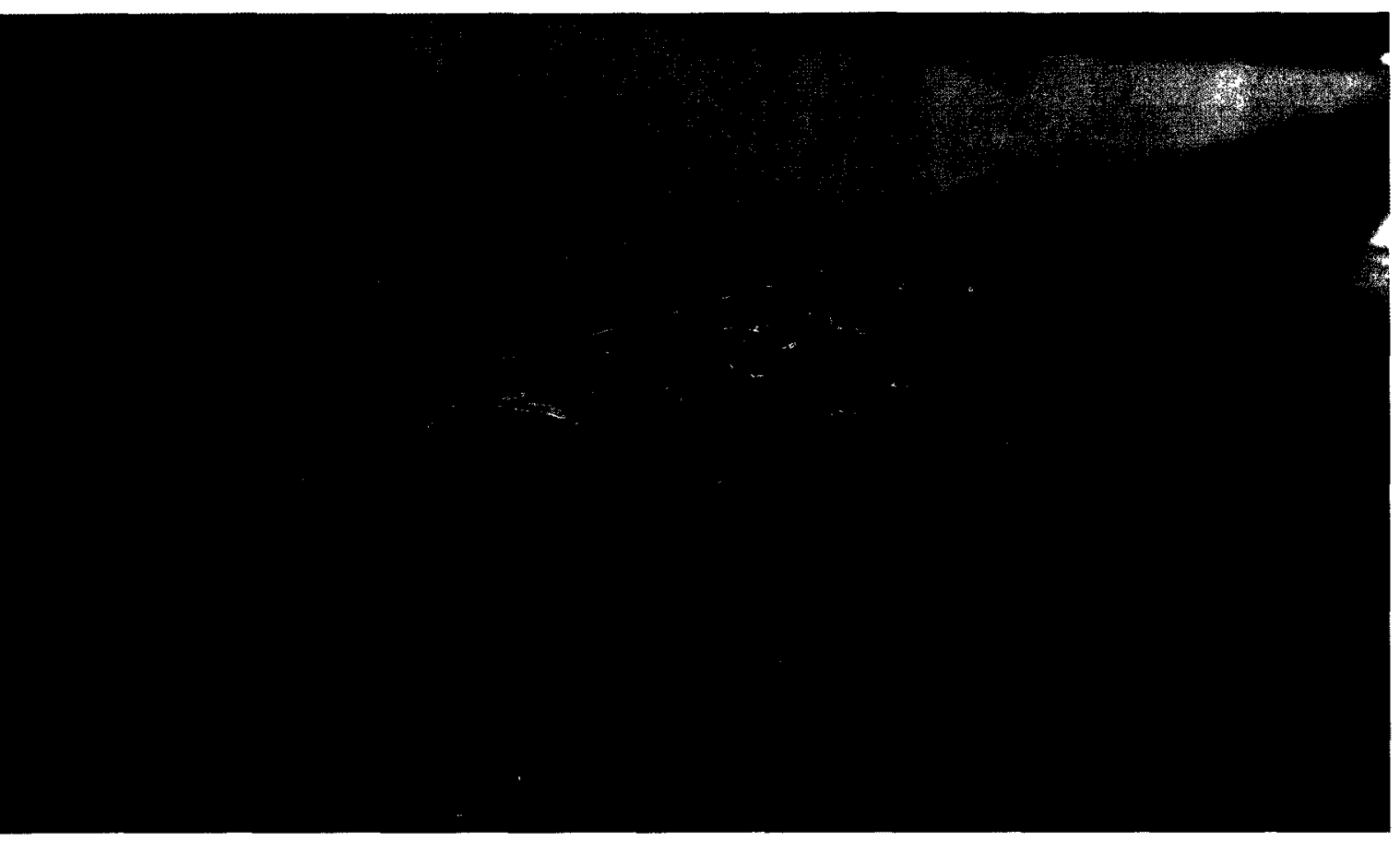

Figure 3.5: Photo of actual high voltage ladders. Visible are both connections for upper and lower cathode boards and $1 \mathrm{M} \Omega$ resisters on circuit wafer.

the current being drawn. The equations for $I_{\text {wire }}$ and $I_{\text {cathode }}$ are given as eqn 3.2 and eqn 3.3 respectively.

$$
\begin{gathered}
I_{\text {wire }}=\frac{V_{\text {anode }}-V_{\text {cathode }}}{25 M \Omega} \\
I_{\text {cathode }}=\frac{V_{\text {cathode }}}{26 M \Omega}
\end{gathered}
$$

In eqn 3.3 the denominator is $26 \mathrm{M} \Omega$ because the total resistance of the $\mathrm{HV}$ ladder is $26 \mathrm{M} \Omega$. These calculable quantities can be compared to what is being drawn by the detector from the HV power supply. There is typically a deviation of a few $\mu \mathrm{A}$ but that arises from uncertainty in the supply's measurement. Deviations of $10 \mu \mathrm{A}$ or more are cause for investigation. 


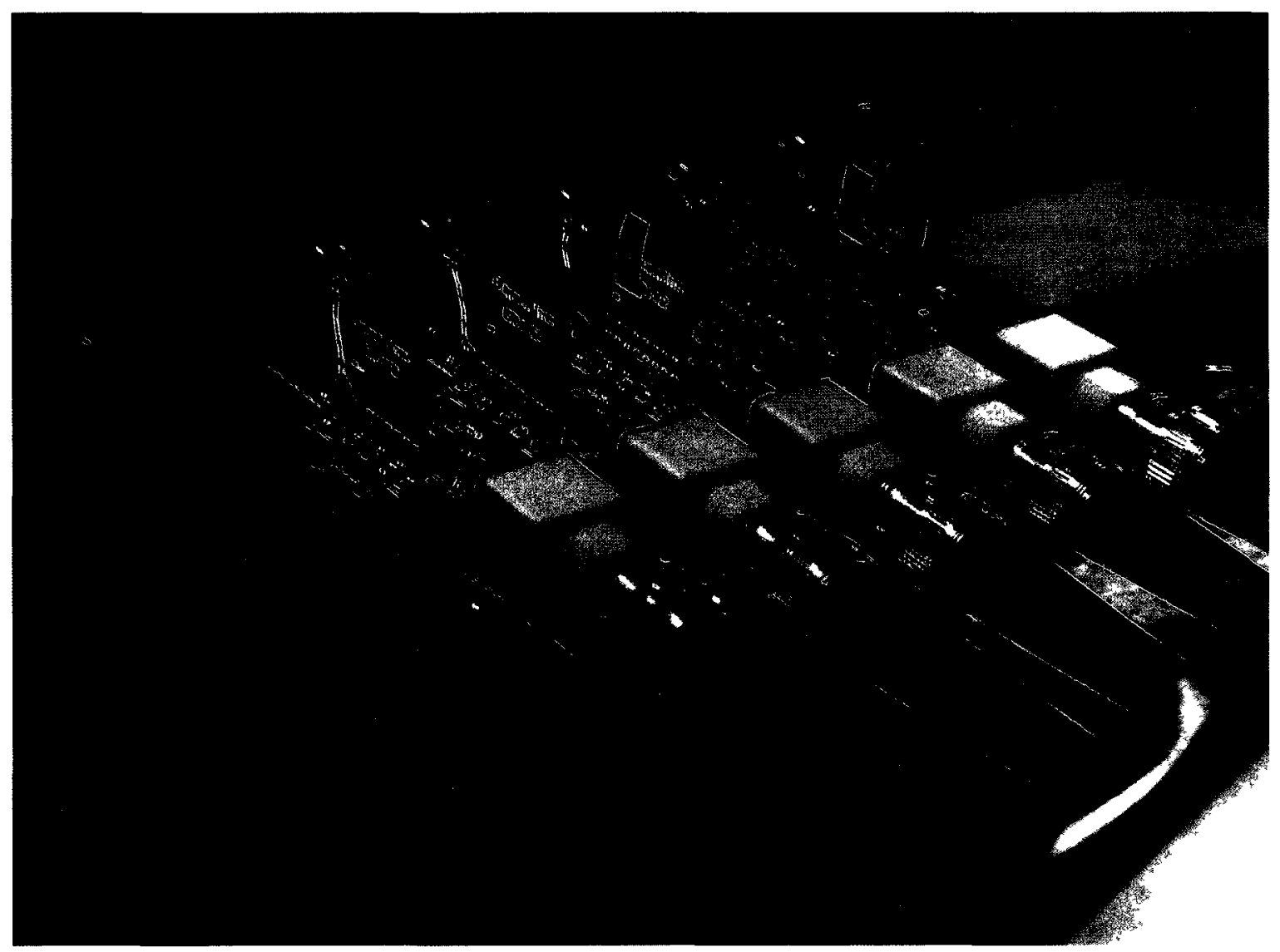

Figure 3.6: Photo of actual preamplifier board. Cremat-112 preamplifiers were used for the research in this thesis

\subsubsection{Signal Processing and Readout Electronics}

Signals from the wire are send by direct connection to the preamplifier board. This board is situated on the opposite side of the detector as the HV boards. The signals are sent through a decoupling capacitor, a diode, then to the preamps for initial shaping. The decoupling capacitor and the diode are placed between the preamps and the anode wire and 'zig-zag' read out in order to protect them from electrical surges created by the deposition of charge on the wire. The actual preamplifier board used is shown in fig 3.6. The Cremat $112^{5}$ charge sensitive preamplifier was chosen because it has a fast rise time and a long decay time constant. The rise time is defined

\footnotetext{
${ }^{5}$ Cremat products manufactured in Newton, MA, USA
} 


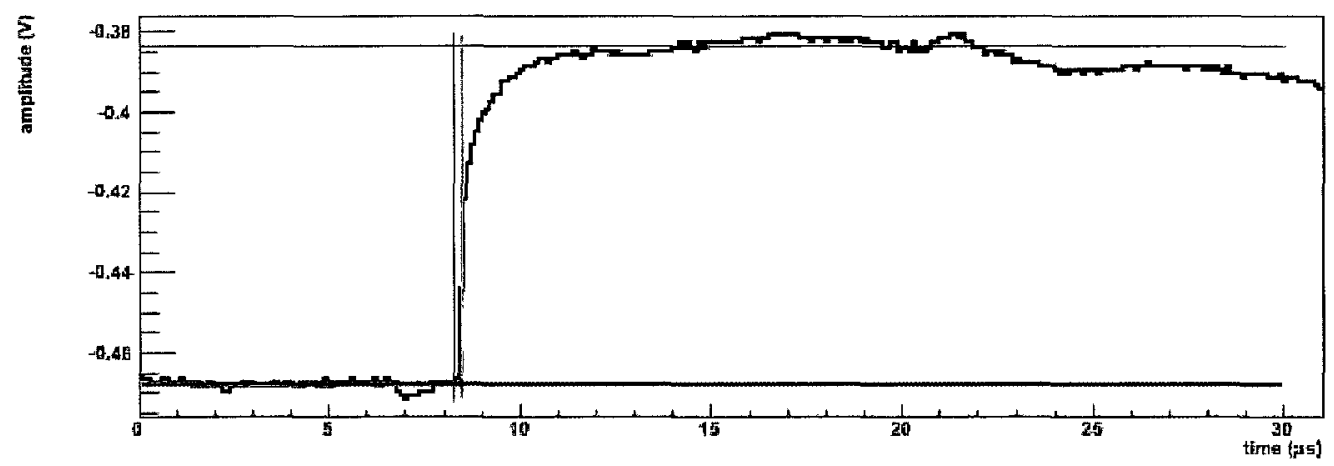

Figure 3.7: A typical pulse from the CRIPT prototype drift chamber preamplifiers. Notice the fast rise and slow decay of the pulse. This is due to the choice of preamplifiers

as the amount of time a pulse takes to go from $10 \%$ to $90 \%$ of its maximum value. The practical rise time is calculated as $\mathrm{t}_{r i s e}=0.25 \mathrm{C}_{D}+6 \mathrm{~ns}$ where $\mathrm{C}_{D}$ is the capacitance of the detector. For the CRIPT prototypes this capacitance is $25 \mathrm{pF}$ resulting in a rise time of $12.25 \mathrm{~ns}$. This preamplifier has a gain of $15 \mathrm{mV} / \mathrm{pC}$ and can detect a maximum of $210 \mathrm{pC}$ of charge per event. This creates pulses with abrupt rises and very long decays and these pulses are then sent to the data acquisition system for processing and storage.

A sample of the pulses obtained from the preamplifier board is shown in fig 3.7. Visible in the plot is a vertical line drawn at the first bin where the pulse appears to rise. This is how the timing of the pulse is determined. The condition for $t_{0}$ is $^{6}$ that the subsequent bins must be filled with larger values than the noise in the signal. Once this condition is met the fit is made and $t_{0}$ can be found.

\section{CRIPT trigger}

Due to the symmetry of the CRIPT drift chambers about their anode wires it was decided for practical reasons to examine only one of the two identical drift regions.

\footnotetext{
${ }^{6} t_{0}$ is the time the event took to drift ionization to the wire
} 
With that restriction in mind it is only necessary then to illuminate that portion of the detector with the cosmic ray telescope. ${ }^{7}$ Fig 3.8 shows a drawing of the CRIPT trigger system - not to scale - but simply the locations of the components relative to each other. It should be noted that fig 3.8 shows the illumination of only a half of the drift volume. To extend this to both sides of the anode wire would simply require producing an identical system for that side.

The top scintillator does not cover the same area as the middle or bottom scintillators. It covers an area of $38 \mathrm{~cm}$ by $60 \mathrm{~cm}$ whereas the middle and bottom both cover $40 \mathrm{~cm}$ by $60 \mathrm{~cm}$. The actual spatial locations of the trigger components - along the vertical axis - are as follows:

- Top Scintillator to wire-plane: $5 \mathrm{~cm} \pm 0.5 \mathrm{~cm}$

- Wire-plane to middle scintillator: $17.64 \mathrm{~cm} \pm 5 \times 10^{-2} \mathrm{~cm}$

- Wire-plane to bottom scintillator: $94.39 \mathrm{~cm} \pm 5 \times 10^{-2} \mathrm{~cm}$

In order to accurately estimate not only the acceptance of the cosmic ray trigger but also the distribution of cosmic ray events inside the the drift chamber itself ${ }^{8}$ a Monte Carlo simulation was written to shed light on this issue.

\section{Cosmic ray muon Monte Carlo}

The first objective of the Monte Carlo (MC) simulation is to determine the acceptance of the trigger system as it was used in the laboratory. In order to do so one must consider a few small details. Firstly, the angular ${ }^{9}$ distribution of cosmic rays which is best described by a $\cos ^{2}$ distribution. The easiest way to generate random numbers from a function is to calculate its cumulative distribution function and sample from

\footnotetext{
${ }^{7}$ Cosmic Ray Telescope is another name for the muon trigger since it "sees" mostly muons

${ }^{8}$ In the plane of the anode wire

${ }^{9}$ with respect to the vertical
} 


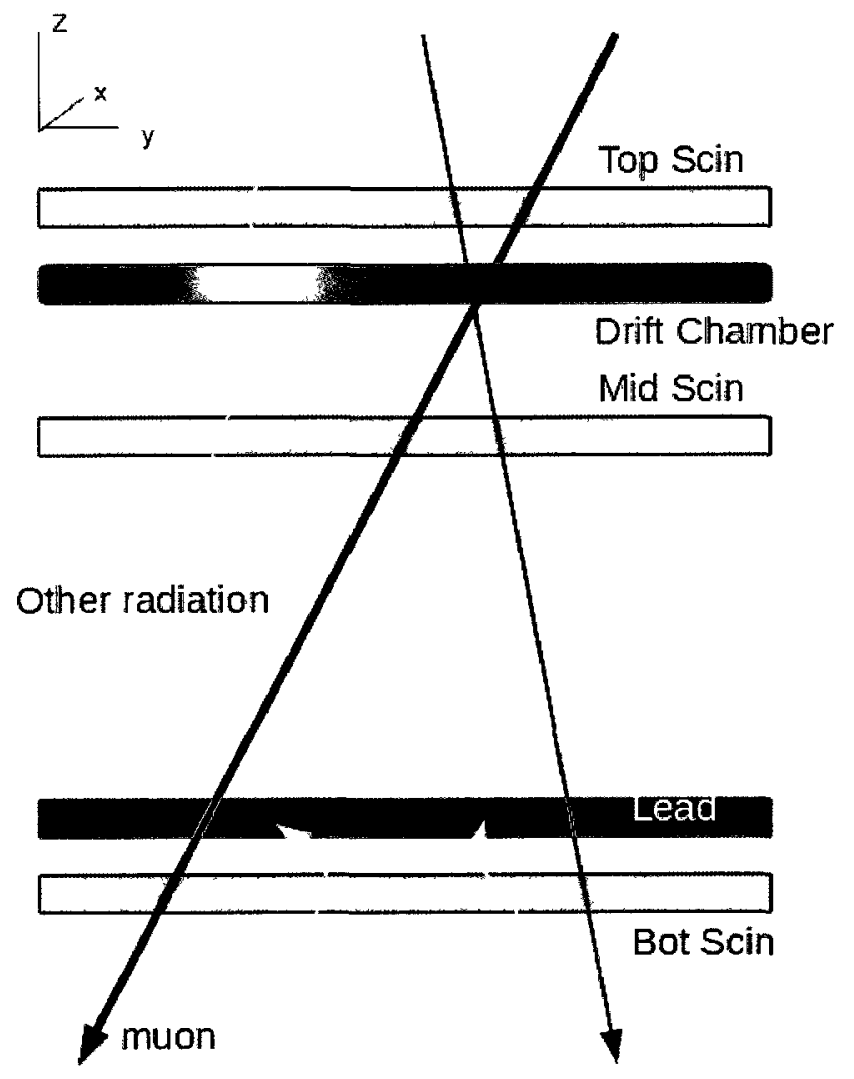

Figure 3.8: CRIPT cosmic ray muon trigger system with drift chamber location shown. 
the inverse. For $\cos ^{2}$ the cumulative distribution function is unfortunately not easily invertible. There are two solutions to this. One being using the ziggurat [25] method for generating random numbers from such distributions. The other is to use a simple ROOT script which samples from the $\cos ^{2}$ function randomly with little effort. The latter was chosen for simplicity. The MC simulation follows the following steps:

- Generate a random position in top scintillator for muons to intersect

- Generate the polar angle from the $\cos ^{2}$ distribution

- Generate the azimuthal angle (in the xy-plane ${ }^{10}$ )

- Generate a value for muon momentum from the BESS momentum spectrum

- Propagate muon track through space with direction cosines

It should be noted that due to the long radiation length of all components involved multiple scattering was ignored along with $\mathrm{dE} / \mathrm{dx}$ for the muons as they pass through all the included materials.

The attenuation by the geometry of the cosmic ray telescope is clearly evident in fig 3.9. It should be noted that the colour scales have been renormalized for each plot (upper left, upper right, lower left). In spite of this, the attenuation can be seen through the statistics of muons that pass through all three scintillators - which is a requirement for them to appear in the lower left plot. The plot shown in the lower right corner is the momentum spectrum seen by all three scintillators. Notice that it is only statistically attenuated which stems from the fact that physical processes were not considered for this geometrical simulation. The attenuation coefficient is calculated by taking the ratio of detected muons in all three scintillators and dividing

\footnotetext{
${ }^{10} \mathrm{z}$-direction is oriented vertically
} 

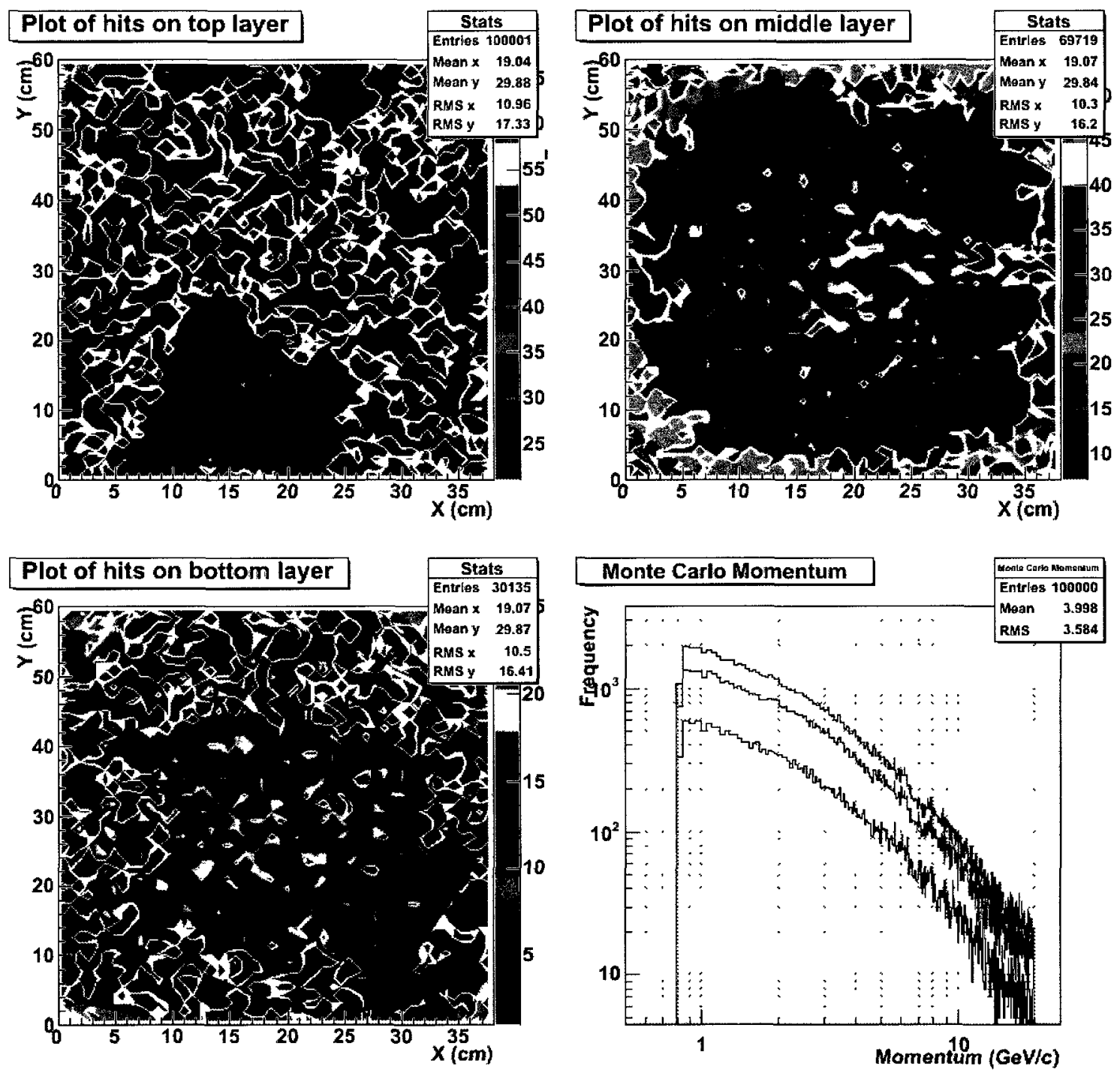

Figure 3.9: Geometrical MC results for tracking muons through three layers of scintillators separated by the distances previously listed. Lower right corner: Blue is the top scintillator, red is the middle, and black is the bottom scintillator's momentum spectrum 
it by the initial number of muons generated by the simulation ${ }^{11}$. This is shown in eqn 3.4 .

$$
\Gamma=\frac{N_{\text {triple }}}{N_{\text {total }}}
$$

This yields an attenuation coefficient of 0.299 to the number of incident events. The cosmic flux of 1 muon per $\mathrm{cm}^{2}$ per min per steradian can be calculated knowing the solid angle of the telescope. This solid angle is $0.0197 \mathrm{sr}$ which implies that the detectable rate should be approximately $0.8 \mu / \mathrm{s}$.

The second and important role of the geometrical MC is to determine the spread of muon tracks through the detector in the plane of the anode wire. This can be done in exactly the same way as the previous flux estimations for the three scintillators. The important part is to know how far the tracks must be projected from the top scintillator to the plane of the anode wire inside the detector. The MC results for this are shown in fig 3.10. It can easily be seen that the most frequently penetrated region is the one illuminated by the center of all three scintillators.

\section{Data acquisition system (DAQ)}

The DAQ was custom made for the CRIPT drift chambers. It is a Labview ${ }^{12}$ based bridge between the hardware transferring the pulses from the detector and the software that records it. The hardware limitations are that it has a maximum sampling rate of $10 \mathrm{MHz}$ at 12 bits and can record information from 16 channels which are then fed through an analogue to digital converter (ADC). This sampling rate is much higher than is required to record events based on signals from the trigger scintillators. The software designed to accommodate the signals uses the continuous sampling clock which has a resolution of $100 \mathrm{~ns}$. That is to say that there is a 100 ns uncertainty

\footnotetext{
${ }^{11}$ All generated events pass through the top scintillator

${ }^{12}$ National Instruments is based in Austin, Tx, USA
} 

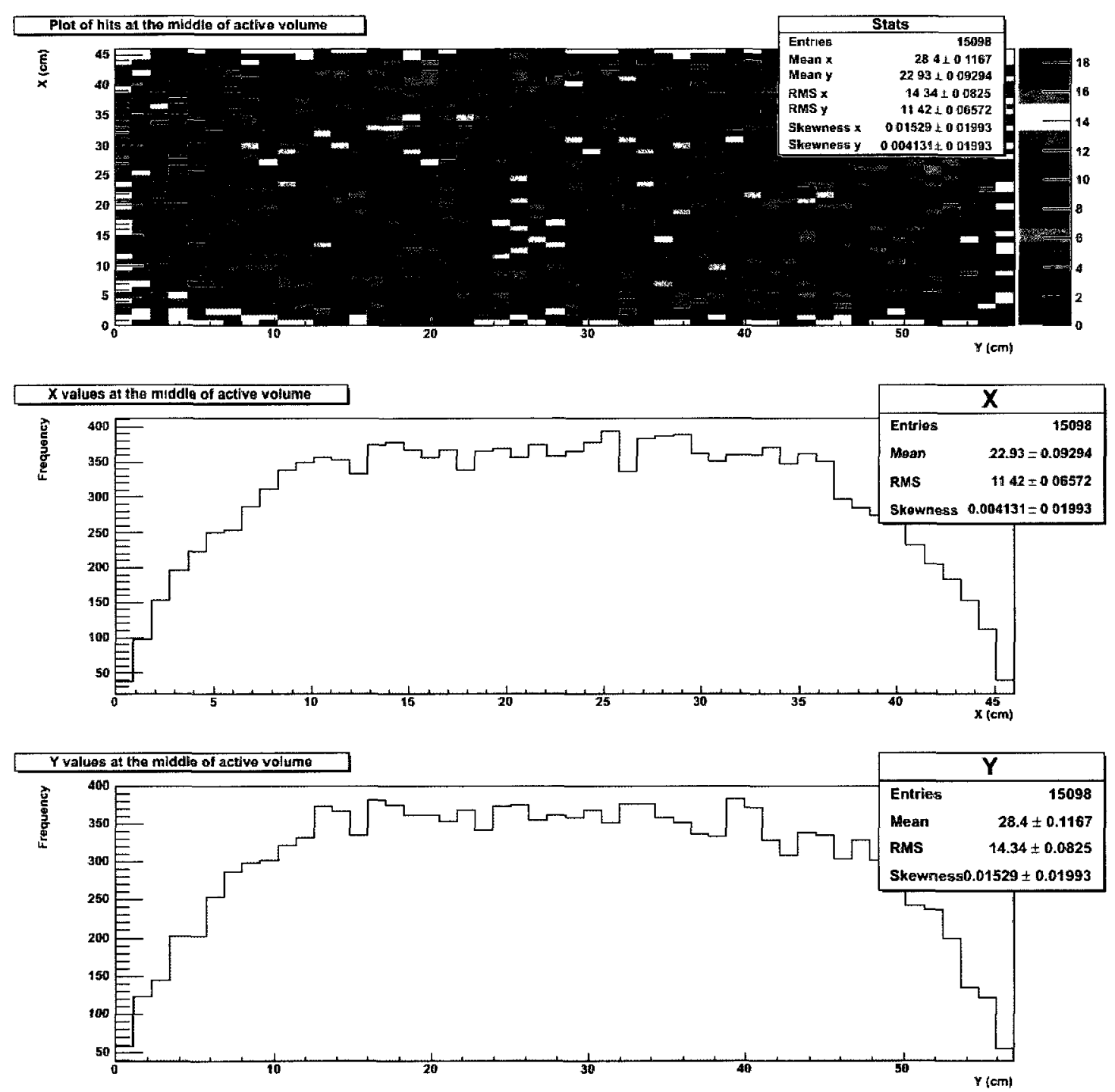

Figure 3.10: Top: Hits in both $\mathrm{X}$ and $\mathrm{Y}$ with frequency denoted by colour. The most probable hit position region is in the center of the drift area. Middle/Bottom: Projections of the top plot onto the $\mathrm{X}$ and $\mathrm{Y}$ axis respectively 
on the time the DAQ assigns a trigger pulse to have arrived. One important feature of the DAQ is that it performs continuous sampling in a loop. If no trigger pulse is detected the loop overwrites the memory buffer and continues. When the trigger pulse arrives the DAQ, having already been sampling, can record not only an amount of time after the pulse, but it can record information before the trigger. Presampling is useful when attempting to resolve ambiguity around when a pulse was actually detected as opposed to when the software says it was. For a single drift chamber there are 5 output channels sending pulse information, voltage and time, to the DAQ. If the event is to be kept the DAQ software saves each event as its own binary file for later manipulation by analysis code. Although not used for this thesis, the DAQ can also determine absolute voltage values for pulse heights. This is of paramount importance when measuring the amount of charge deposited on the anode and cathode.

\subsection{Drift gases}

The choice of drift gas depends upon the requirements of the detector it is used in. As previously discussed, different gases have vastly different properties. Moreover, different mixtures of gases will behave quite differently over a range of electric fields. For the CRIPT prototypes, the most important requirement is a small position resolution and the smallest attainable resolution will depend on the choice of gas for both coordinate measurements. The resolution is effected by the transverse and longitudinal diffusion as well which depends on the type of gas. These effects typically become smaller as the electric field strength becomes larger as seen in fig 3.11. The ranges of electric field are limited by the hardware used to fabricate the detectors. All that is to say that there are many limiting factors regarding the best possible resolution of a detector.

The work in this thesis considered four gas mixtures in total. They are: 
- argon-methane: 90\%-10\%

- argon-ethane: $90 \%-10 \%$

- argon-carbon dioxide: $85 \%-15 \%$

- argon-ethane-acetylene: $90 \%-5 \%-5 \%$

Each gas mixture will perform uniquely in the CRIPT prototypes but in order to confirm this actual macroscopic measurements, of drift velocity for example, must be made and this is what this thesis aims to do. The methods employed to make these measurements will be explained in a subsequent section. The drift fields under which these gases were examined are $53 \mathrm{~V} / \mathrm{cm}, 70 \mathrm{~V} / \mathrm{cm}$, and $105 \mathrm{~V} / \mathrm{cm}$. It should be noted that not all gases were examined under all drift fields. $\mathrm{Ar}-\mathrm{C}_{2} \mathrm{H}_{6}$ was examined in all three fields whereas $\mathrm{Ar}-\mathrm{CO}_{2}$ was only examined at $105 \mathrm{~V} / \mathrm{cm}$ because it is already a slow gas and the lower drift fields proved to yield overly low drift velocities. So low in fact that event pileup made taking data impossible. The remainder of the gases were examined under both $70 \mathrm{~V} / \mathrm{cm}$ and and $105 \mathrm{~V} / \mathrm{cm}$. An overview of the general transport properties of these gases is shown in fig 3.11.

The CRIPT gas delivery system is both simple and reliable. The bottle containing the preferred gas mixture is attached to the CRIPT tower and connected to the detector through a regulator and tubing system. There is also a flow-rate meter between the chambers and the regulator to ensure that the flow of gas is kept to a reasonable rate so as not to increase the pressure inside the detector to above atmospheric pressure. As all the gases used are completely transparent to visible light one must ensure that the gas is flowing through the conduction system without obstruction. Each detector also has an oil filled bubbler situated between the gas exit manifold and the atmosphere. This not only ensures gas flow but it also prevents the atmosphere ${ }^{13}$ from entering the chamber and contaminating the gas mixture.

\footnotetext{
${ }^{13}$ Nitrogen, oxygen, and water vapour for example
} 

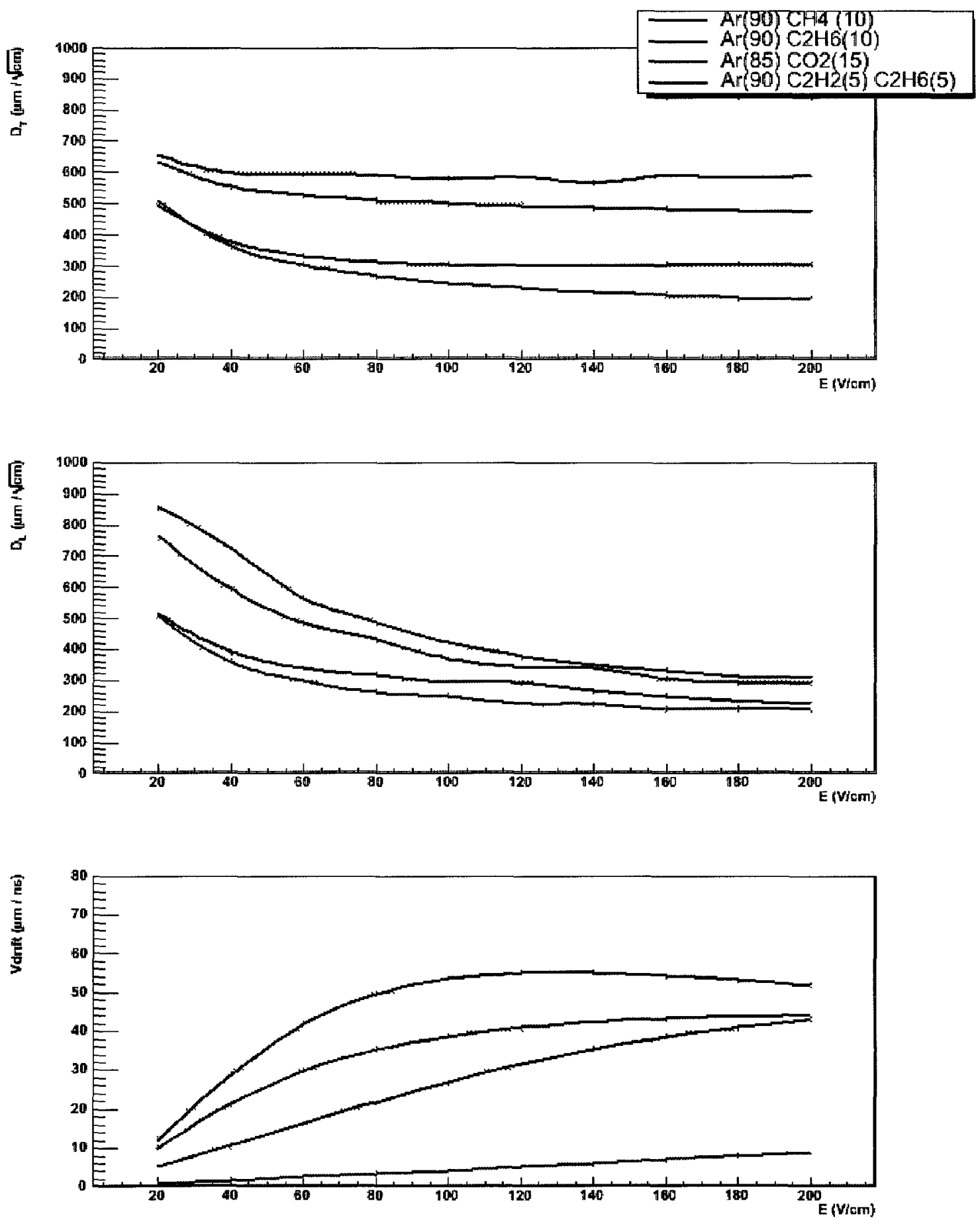

Figure 3.11: Transverse dıffusıon (top), longıtudınal dıffusıon (mıddle), and drift velocity (bottom) from MAGBOLTZ 


\subsection{Drift chamber simulation}

Dr. Boudjemline created a sophisticated electrostatic simulation of the CRIPT drift chambers using successive over relaxation. This method relies on the fact that the solution to Poisson's equation is harmonic. That is to say that the value of potential at some point is equal to the average of the potential at the neighbouring points. Consider Laplace's equation in 2 spatial dimensions given in eqn 3.5

$$
\frac{\partial^{2} \phi}{\partial x^{2}}+\frac{\partial^{2} \phi}{\partial y^{2}}=0
$$

If one then considers the points in an 'xy'-grid around the central point that consist of a 'diamond' about that point and expand in a Taylor series one obtains:

$$
\begin{aligned}
& \phi(x, y+\partial y)=\phi(x, y)+\frac{\partial \phi}{\partial y}(+\partial y)+\frac{1}{2 !} \frac{\partial^{2} \phi}{\partial y^{2}}(+\partial y)^{2}+\frac{1}{3 !} \frac{\partial^{3} \phi}{\partial y^{3}}(+\partial y)^{3}+\ldots \\
& \phi(x, y-\partial y)=\phi(x, y)+\frac{\partial \phi}{\partial y}(-\partial y)+\frac{1}{2 !} \frac{\partial^{2} \phi}{\partial y^{2}}(-\partial y)^{2}+\frac{1}{3 !} \frac{\partial^{3} \phi}{\partial y^{3}}(-\partial y)^{3}+\ldots \\
& \phi(x+\partial x, y)=\phi(x, y)+\frac{\partial \phi}{\partial x}(+\partial x)+\frac{1}{2 !} \frac{\partial^{2} \phi}{\partial x^{2}}(+\partial x)^{2}+\frac{1}{3 !} \frac{\partial^{3} \phi}{\partial x^{3}}(+\partial x)^{3}+\ldots \\
& \phi(x-\partial x, y)=\phi(x, y)+\frac{\partial \phi}{\partial x}(-\partial x)+\frac{1}{2 !} \frac{\partial^{2} \phi}{\partial x^{2}}(-\partial x)^{2}+\frac{1}{3 !} \frac{\partial^{3} \phi}{\partial x^{3}}(-\partial x)^{3}+\ldots
\end{aligned}
$$

Taking the sum of eqn 3.6 , eqn 3.7 , eqn 3.8 , eqn 3.9 then dividing by 4 , working 
with the concept that all points here lie on a sphere centred at $(\mathrm{x}, \mathrm{y})$, implies that $\mathrm{dx}=\mathrm{dy}$ results in the following elegant solution:

$$
\frac{\phi(x, y+d y)+\phi(x, y-d y)+\phi(x+d x, y)+\phi(x-d x, y)}{4}=\phi(x, y)
$$

Eqn 3.10 is exactly the relationship required to perform successive over relaxation $^{14}$. Given this mathematical relationship, the voltage of the anode and central cathode strips, and setting the potential at the aluminum side rails to zero allows the simulation to calculate the value of the electric field at all points inside the detector. This was implemented with a box (or step) size of $1 \mathrm{~mm}$ for the exact physical layout of a CRIPT drift chamber. The component of the simulation that takes MAGBOLTZ's calculated relationship between electric field and drift velocity and relates the field inside the detector to a velocity as a function of distance makes the assumption that the fastest electrons are always in the plane of the anode wire. This is not the case for a real ionization inside the detector but it is a reasonable assumption that introduces very small uncertainty to the simulated data. For that reason, the simulation will be assumed to acquire its error directly from the statistical uncertainties produced by MAGBOLTZ.

The simulated electric field is shown in fig 3.12 and is calculated without the presence of the drift gas. It should be noted that the vertical scale (height) is greatly exaggerated in this plot. This mapping of the field allows for the tracks of electrons to be simulated through the chamber as well. Fig 3.13 is a zoomed version of the top plot in fig 3.12 and shows that the electric field in the region very close to the anode wire is symmetric in all directions except for radially outward.

\footnotetext{
${ }^{14}$ For a complete explanation of this technique please see [26]
} 

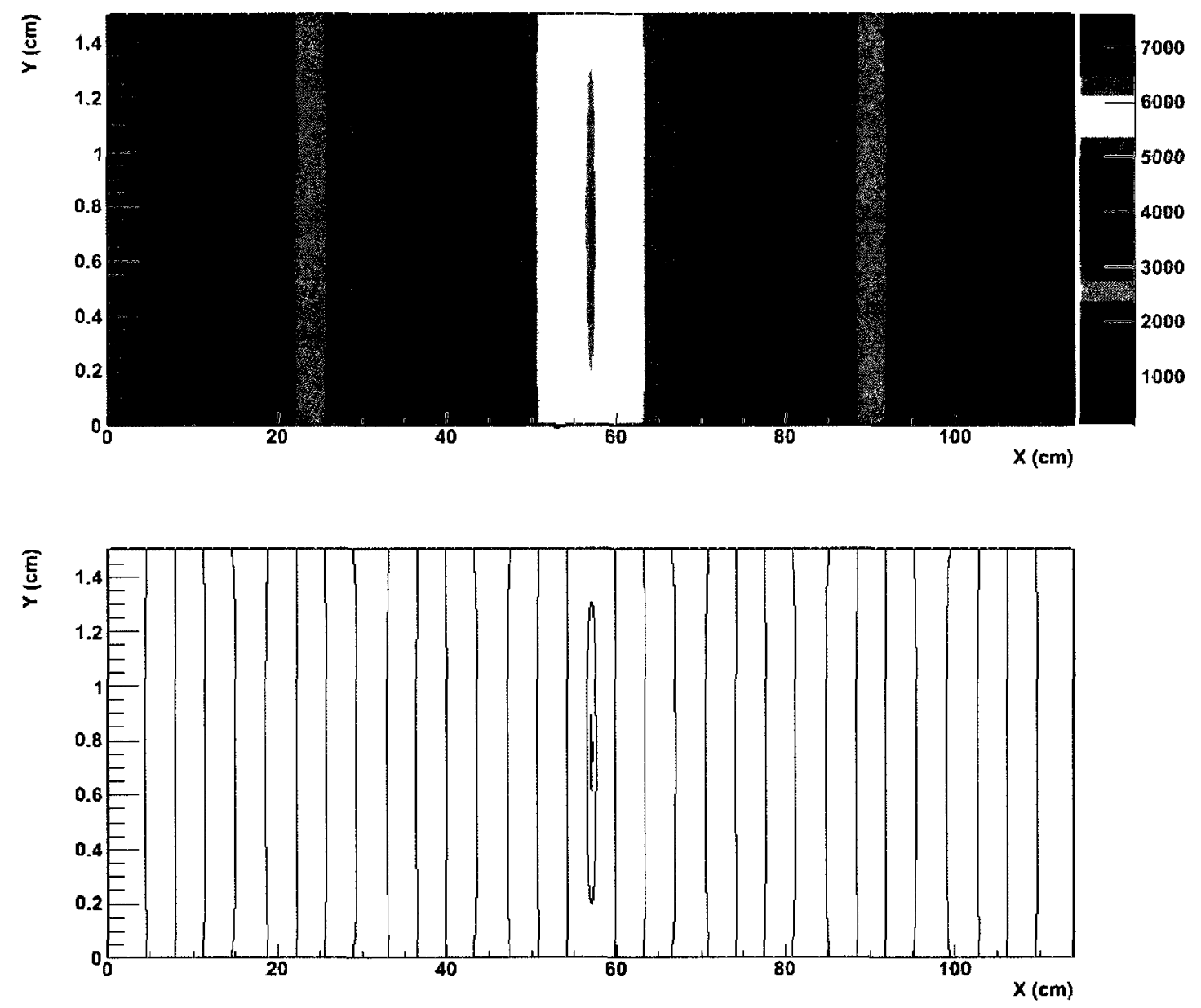

Figure 3.12: Successive Over Relaxation (SOR) results for the electric field inside a CRIPT drift chamber. Bottom: Field lines resulting from the SOR calculation. The choice of axis is arbitrary for this diagram. As a point of reference the anode wire would run into and out of the page. The units for the colour scale are $\mathrm{V} / \mathrm{cm}$. 


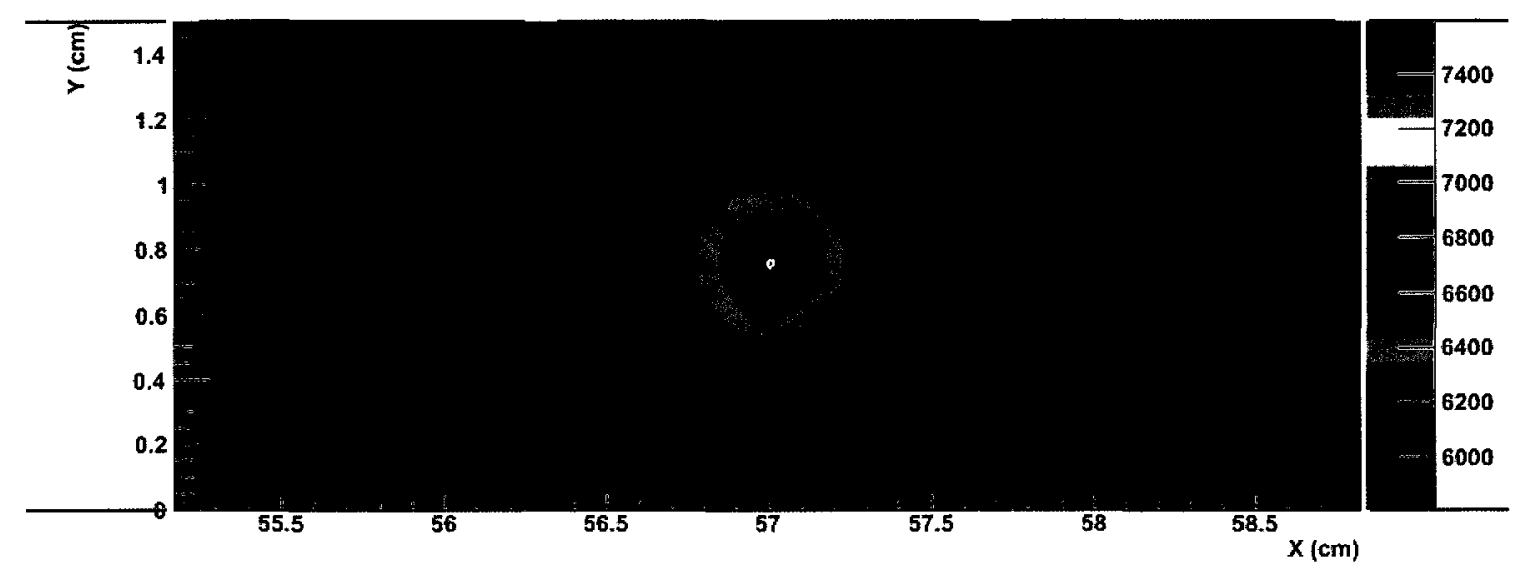

Figure 3.13: Close-up of anode wire and resulting field. This is the gas gain region where ionization avalanches occur. The units for the colour scale are $\mathrm{V} / \mathrm{cm}$.

One effect observed in the simulation for electron tracks through the field is oscillations when the track is near to the cathode boards. The CRIPT cathode boards have a $0.1 \mathrm{~cm}$ gap between electrodes. This causes localized changes in the equipotential lines inside the detector and is responsible for the oscillatory nature of the flight path. Fig 3.14 shows how the electric field changes when very close to the gaps in the cathode board. The gap size was chosen as such because over time exposed G10 can contaminate the drift gas and attenuate signals from the detector. With such a small gap, the total exposed area is so small that this detrimental effect can essentially be neglected.

Figs 3.15 and 3.16 demonstrate the difference in flight paths experienced by electrons both near the anode plane and near the cathode board respectively. It should be noted again that the vertical scale is not proportioned with respect to the horizontal one so the oscillations, though obvious, are greatly exaggerated.

The simulation, when given the MAGBOLTZ output of electric field and drift velocity, produces the plot shown in fig 3.17. These velocities result from tracks lying in the plane of the anode wire inside the detector. The simulation, when calculating 

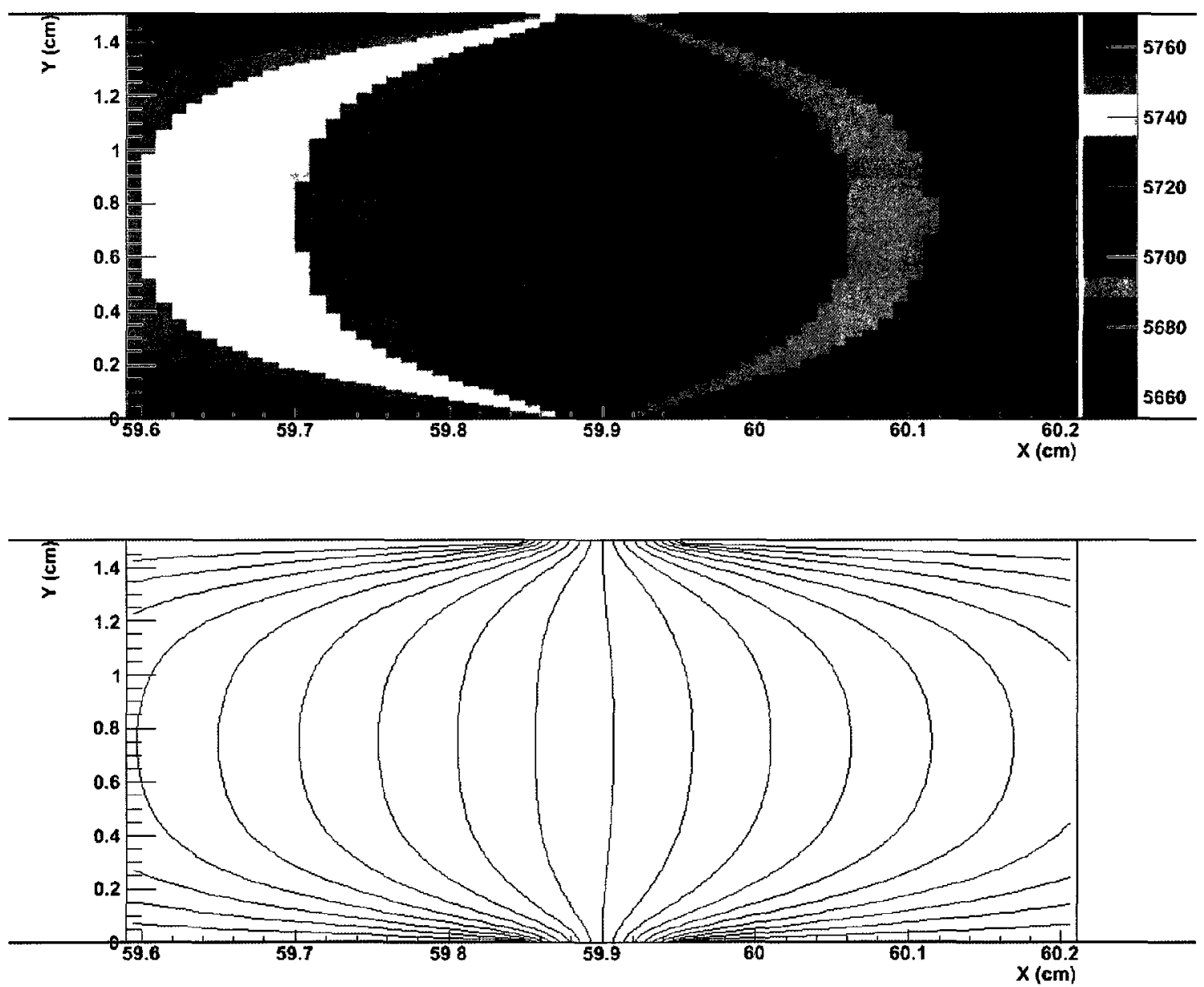

Figure 3.14: An enhanced view of how the electric field changes when very close to the gaps in the cathode boards. The units for the colour scale are $\mathrm{V} / \mathrm{cm}$. 


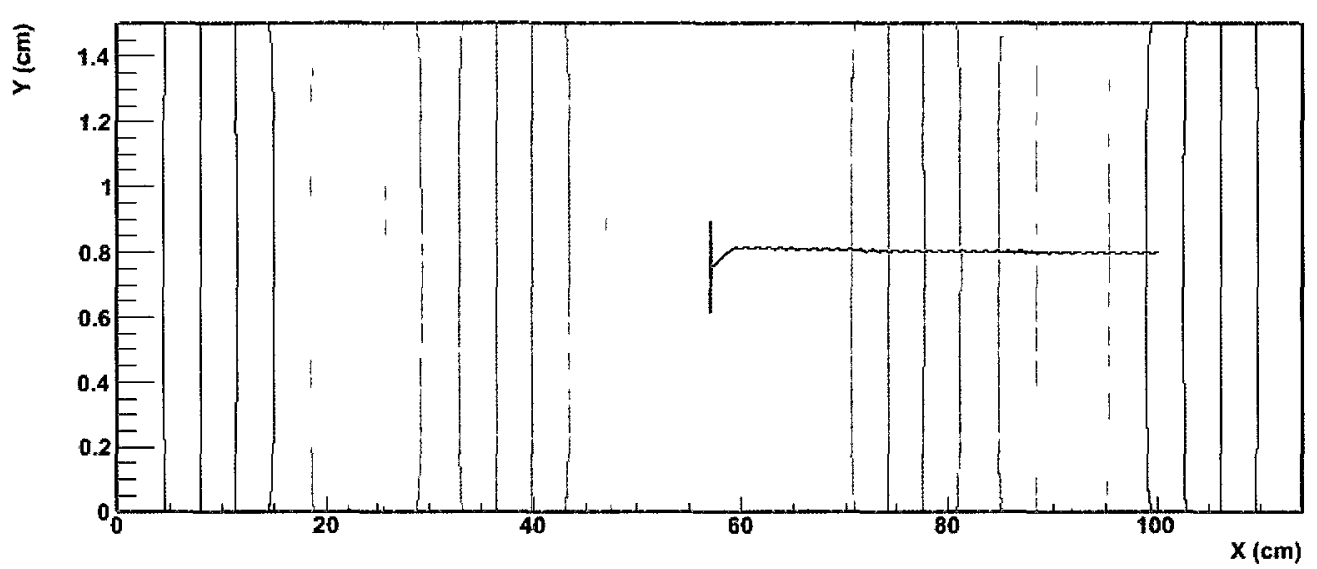

Figure 3.15: Simulated flight path for an electron near the anode plane.

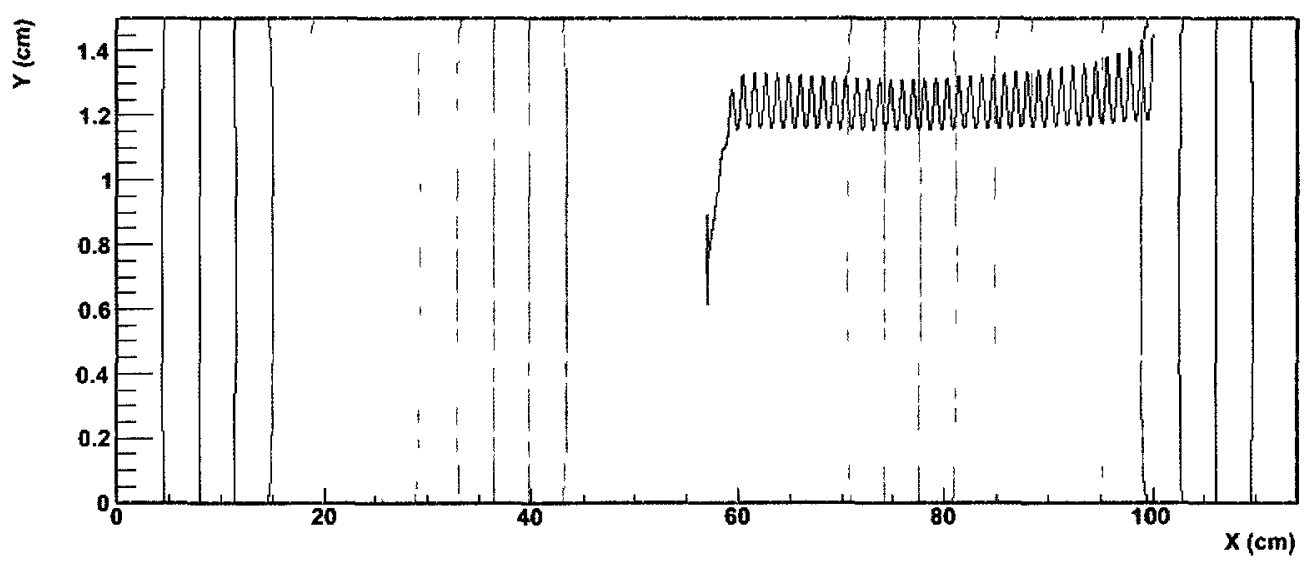

Figure 3.16: Simulated flight path for an electron near the cathode board. 


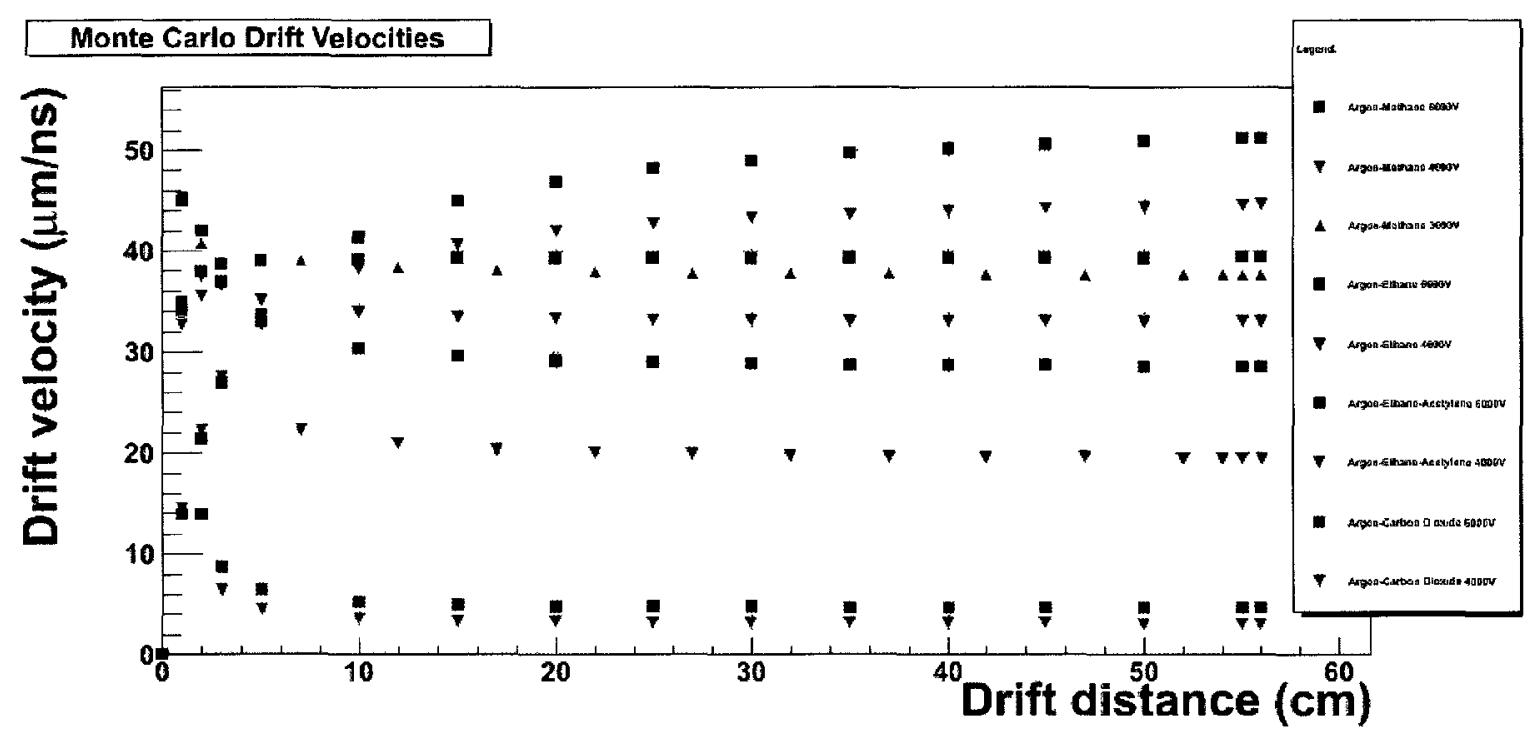

Figure 3.17: SOR and MAGBOLTZ results for drift velocity in the CRIPT prototypes. Different gases are denoted by different colours. $\mathrm{ArCH}_{4}$ is in black, $\mathrm{ArC}_{2} \mathrm{H}_{6}$ is in red, $\mathrm{ArC}_{2} \mathrm{H}_{2} \mathrm{C}_{2} \mathrm{H}_{6}$ is in blue, and $\mathrm{ArCO}_{2}$ is in green. The marker shapes denote the applied cathode voltage. Squares are $6000 \mathrm{~V}$ and triangles are $4000 \mathrm{~V}$.

the velocities, also ignore the diffusion effects as it simulates only a single electron drifting. This is the simplest possible method of simulating the drift chambers and because of this it will be assumed to be the "ideal" circumstances. 


\section{Chapter 4}

\section{Experimental techniques}

When collecting the data for this thesis I employed two techniques for measuring the drift velocity of ionization inside the CRIPT prototype drift chambers. One method I call "Geometrical Discrimination" (GD) and the other "Active Area Reduction" (AAR). They are both unique in their methodology but I will demonstrate that they are both adequate for meeting the goals of this thesis. I will also argue why one was chosen over the other for the majority of the data collection.

\subsection{Geometrical Discrimination}

This technique takes advantage of the fact that the DAQ records data only when triggered by the trigger scintillators. Implementing this method is very simple and straightforward. It involves adding a 4th trigger scintillator to the existing apparatus. The 4th scintillator is situated at the same height as the topmost trigger scintillator and their ends are overlapped by some amount. This amount of overlap can be set to any value short of the maximum length of the scintillators because that would simply be redundant. For the work presented here the overlap was set to $4 \mathrm{~cm}$ with an uncertainty of $0.1 \mathrm{~cm}$. The uncertainty stems from the roughly $0.1 \mathrm{~cm}$ of dead space at the end of each scintillator and the inherent error involved in making measurements 
with a meter stick. It is necessary to adjust the logical trigger to accept coincident signals from 4 scintillators instead of just 3 as it was set originally. This method is shown diagrammatically in fig 4.1. The geometry of the illumination can be seen with the yellow area. Since cosmic ray muons impinge on the detector with an angular distribution which is described by a cosine-squared function, the most probable angle of incidence is $90^{\circ}$ or perpendicular to the horizontal surface of the detector.

In order to collect information about drift velocity over the entirety of the drift length the overlapped scintillators need only be translated along the length of the detector. This is very easy to do and allows for an almost continuous measurement. This method, unlike the alternative, require no adjustments to either the anode or cathode HV settings over the span of a particular data series.

This attenuates the histogram of ionization arrival times in a very convenient way. Instead of the histogram being simply filled uniformly from $t=0$ to $t=t_{\max }$ there will be a roughly Gaussian distribution of arrival times. The quantity ' $\mathrm{t}_{\max }$ ' is the maximum arrival time the ionization can have. It depends on the gas and the applied electric field since the maximum drift length does not change. The faster gases will have shorter maximum drift times than the slower ones as ones' intuition would suggest. An unattenuated timing histogram for an arbitrary gas at arbitrary drift field is shown in fig 4.2. The information shown in the histogram is the drift time for the ionization created by cosmic ray muons. The value for ' $t 0$ ' is determined from the pulse shapes shown in fig 3.7. The number of events detected depends solely on how long the DAQ is allowed to run. Theoretically it can run until there is simply no longer any remaining disk space available.

The geometrical Monte Carlo used to estimate the areas of highest illumination in the scintillators and the drift chamber can be used to make an estimate about the shape of the attenuated timing histogram. The only necessary modification is to ensure that events are taken at the proper distance from where they are generated. 


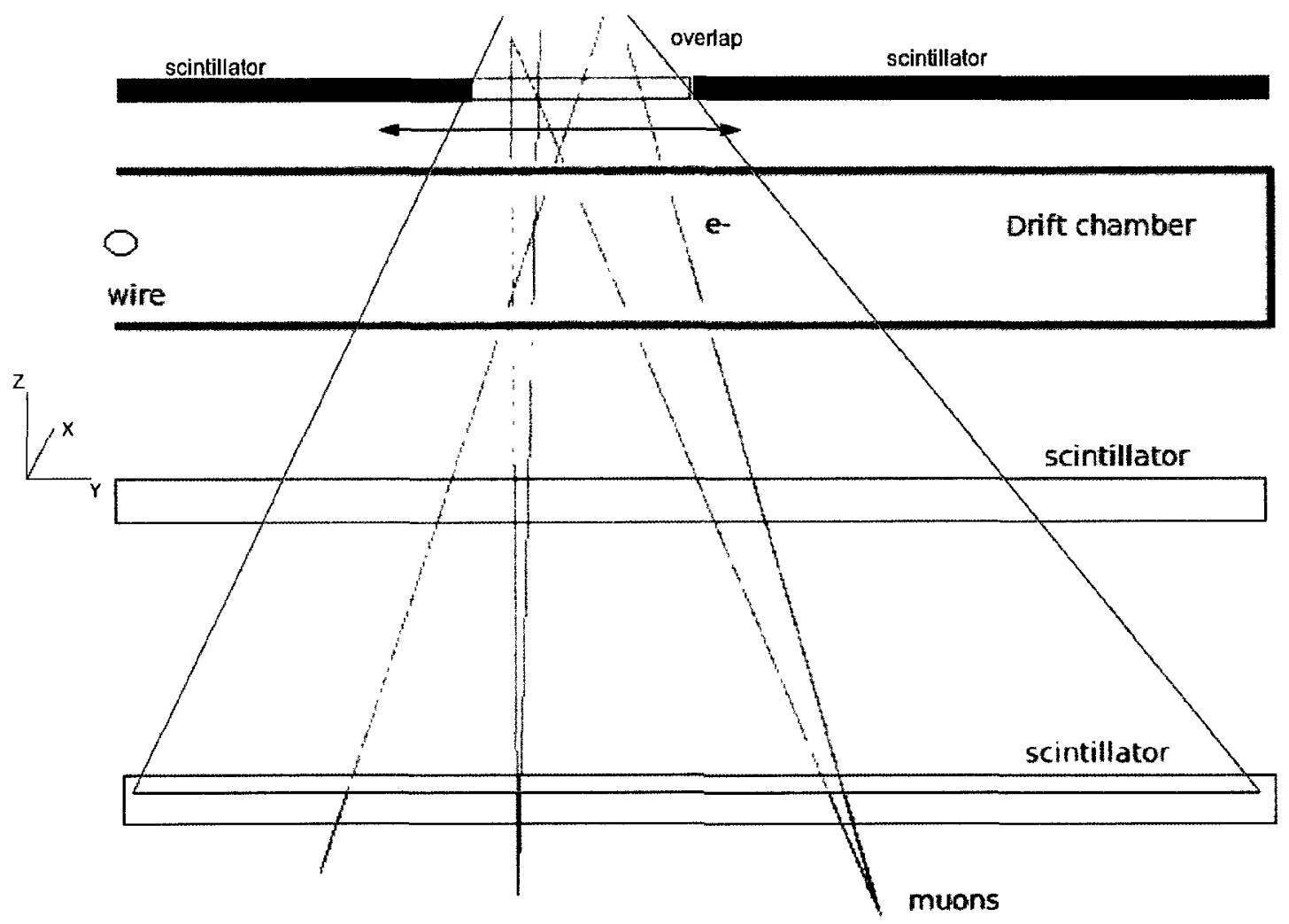

Figure 4.1: A cross-sectional view of the experimental apparatus. Shown are the overlap of the two top most scintillators, half of the detector, and the other two trigger scintillators. The entirety of both top scintillators is not shown because the area that is not used in the overlap does not contribute to the illumination of the detector. 


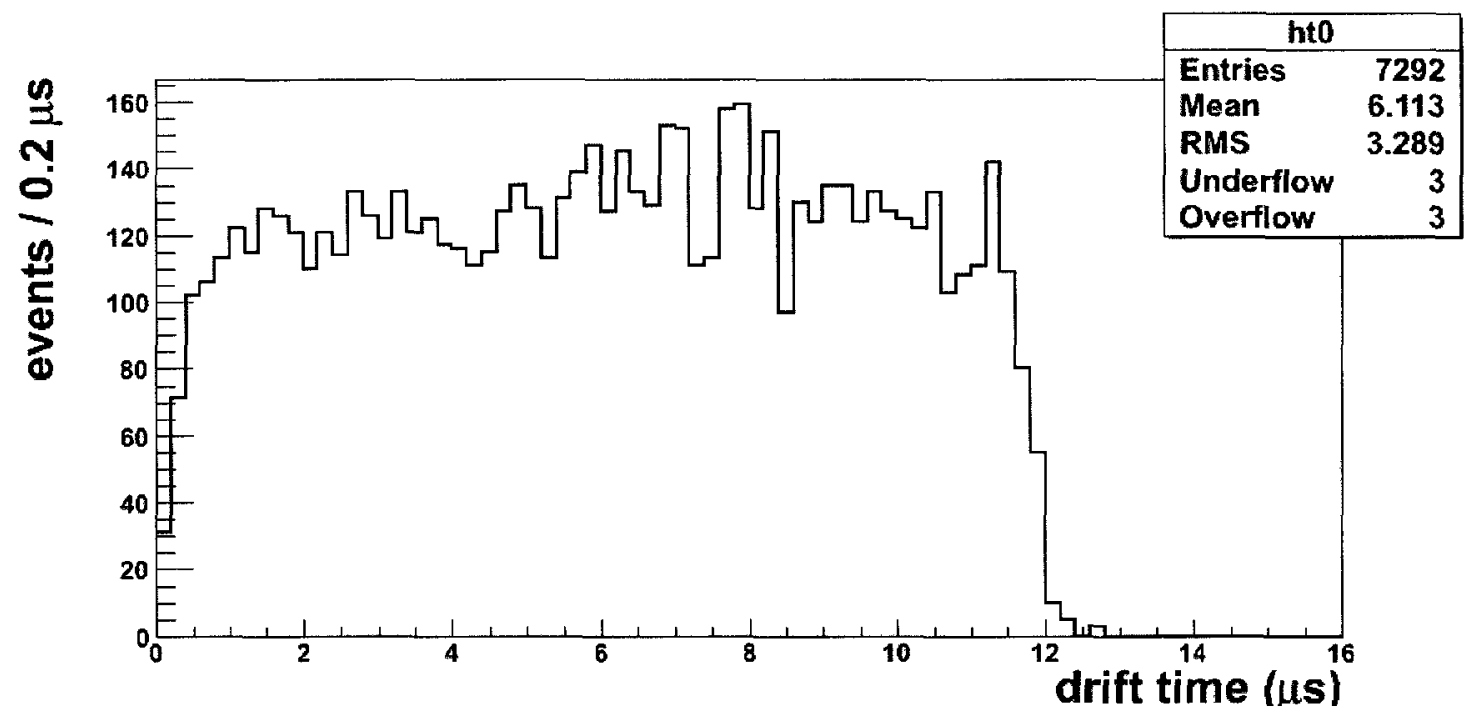

Figure 4.2: An example of a typical timing distribution obtained from the CRIPT drift chambers and DAQ. The distribution is the flight time of the fastest ionization to create a signal on the anode wire.

The simulated GD timing histogram is shown in fig 4.3. For this simulation, events are generated only where the two scintillators are overlapping. The broadening seen is purely a geometrical effect.

The attenuated timing histograms typically appear as shown in fig 4.4. The most effective way of extracting the pertinent information from them is to fit them with a Gaussian and take the mean of the Gaussian as the time observed. This can be correlated with the center of the $4 \mathrm{~cm}$ overlap of the top most trigger scintillators. Having both distance and time allows one to very easily calculate the macroscopic drift velocity.

Another intuitive notion is that the further the tracks must be projected down the more broad the Gaussian will become. This is a very straightforward hypothesis to test as well as it again stems from purely geometrical factors. The relationship between the number of events simulated and the vertical distance between the overlapping trigger scintillators is shown in fig 4.5. Fig 4.5 plots the standard deviation of the simulated data as a function of distance above the anode plane. The data points 


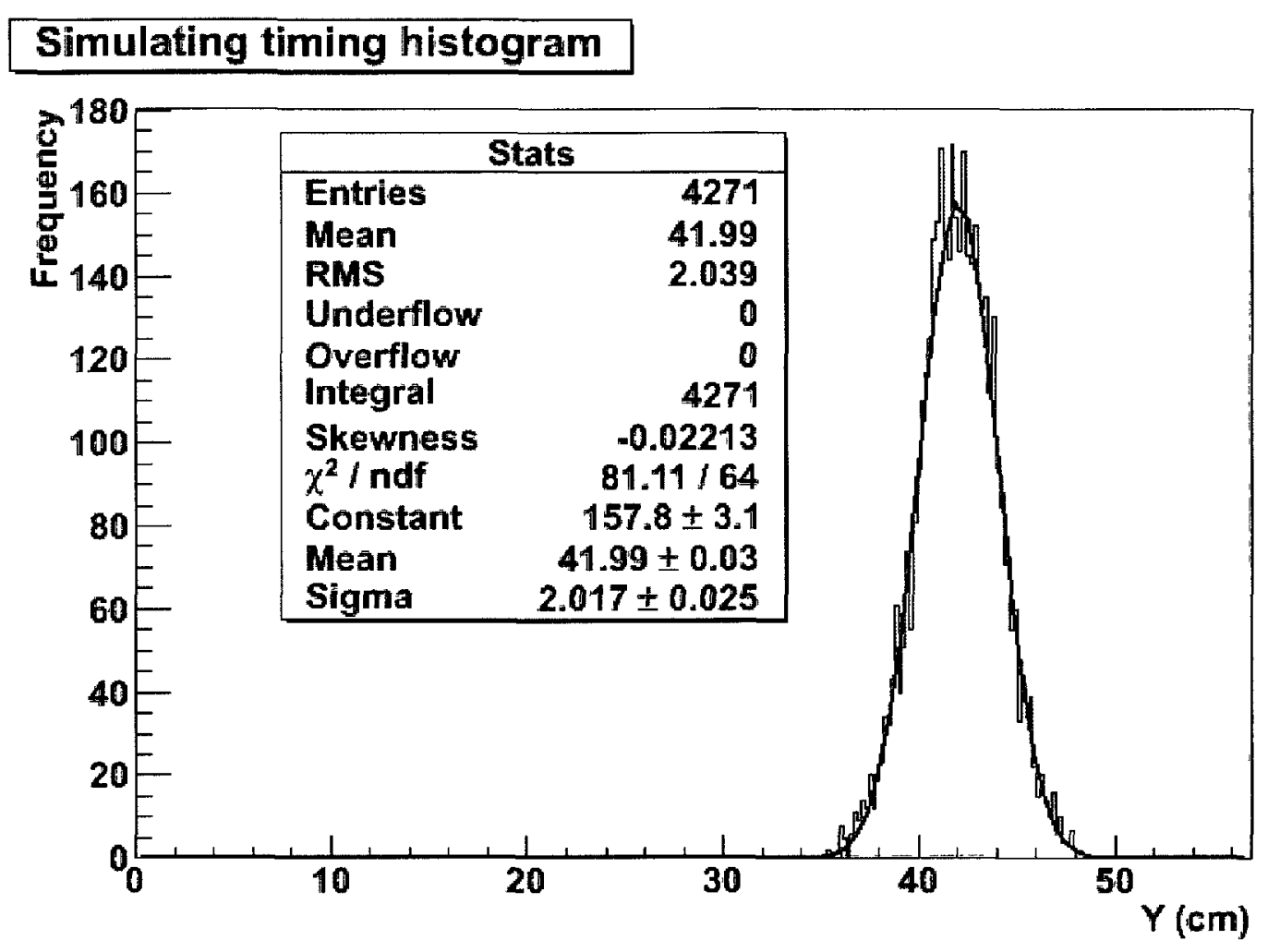

Figure 4.3: Simulated GD timing histogram. Events are projected into the plane of the anode wire and the mean can be extracted.

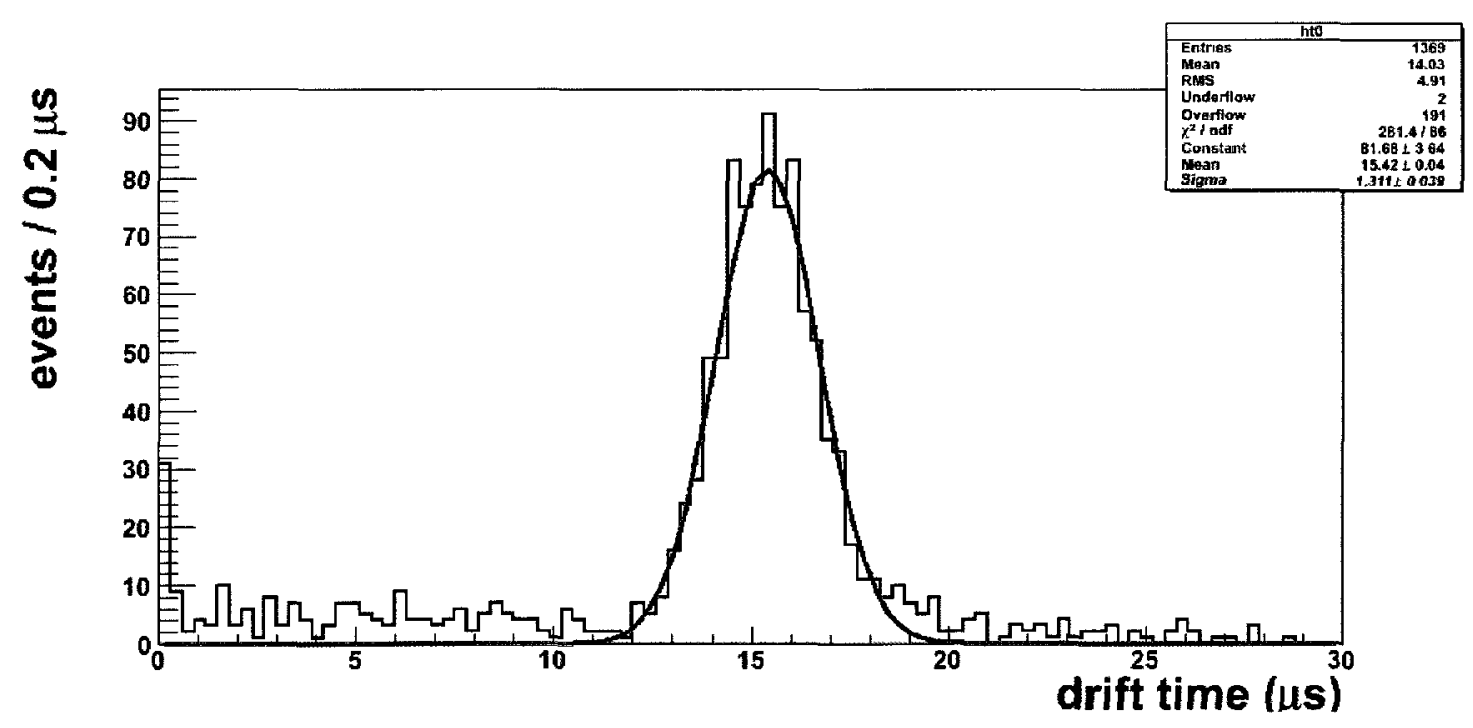

Figure 4.4: An example of a typical timing distribution obtained from the implementation of the GD method. 

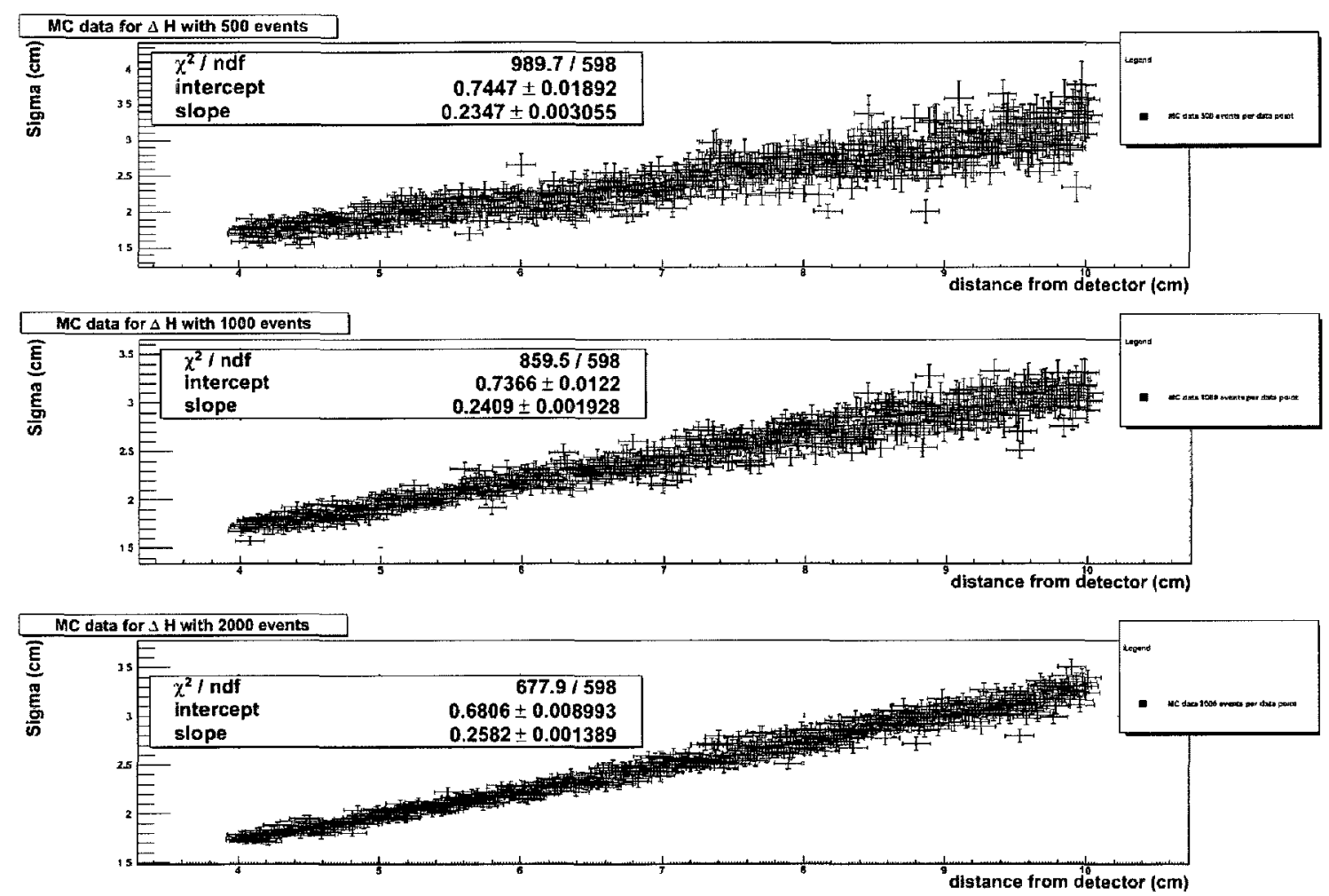

Figure 4.5: Top: 500 events are generated for each data point. Middle: 1000 events are generated for each data point. Bottom: 2000 events are generated for each data point. 
in the top plot stem from a Gaussian generated with only 500 events per data point. The middle and bottom plots are created in the exact same manner differing only by the number of random events used to generate each data point. Not visible is a linear fit to the spread of data points in each plot. The slopes of each fit are essentially identical however the quality of the fit suffers with a larger spread in the data. This clearly highlights the fact that with this method it is beneficial to take above 1000 events for every measurement of drift velocity. The reduced chi-squared for each of the three plots, from top to bottom, are $1.65,1.43$, and 1.13 respectively.

\subsection{Active Area Reduction}

This is the second technique employed in my work of measuring the drift velocity in the CRIPT prototypes. This method, as far as this author is aware, is novel in its application. Geometrical discrimination relied on changing the illumination of the drift area in order to isolate particular parts of the drift chamber. This method is essentially the opposite in that it is the detector that is "modified" in order to gain knowledge of the drift velocity over the entirety of the drift length. As seen in fig 3.5 the individual cathode strips are very easily accessible once the covers are removed from the boards. The essence of this method is to ground a particular cathode strip which effectively reduces the maximum drift length the ionization can traverse. Each cathode strip is a fixed distance from the anode wire and both the electrode width and gap are well defined. The one significant modification that must be made is to the applied HV for the anode and cathode. The drift field inside the detector is an explicit function of the maximum drift length. That is to say as the length is reduced the cathode voltage must in turn be reduced to maintain a consistent field. Modifying the cathode voltage value in turn means that the anode voltage must also be modified. This is because with the GD method the difference between 


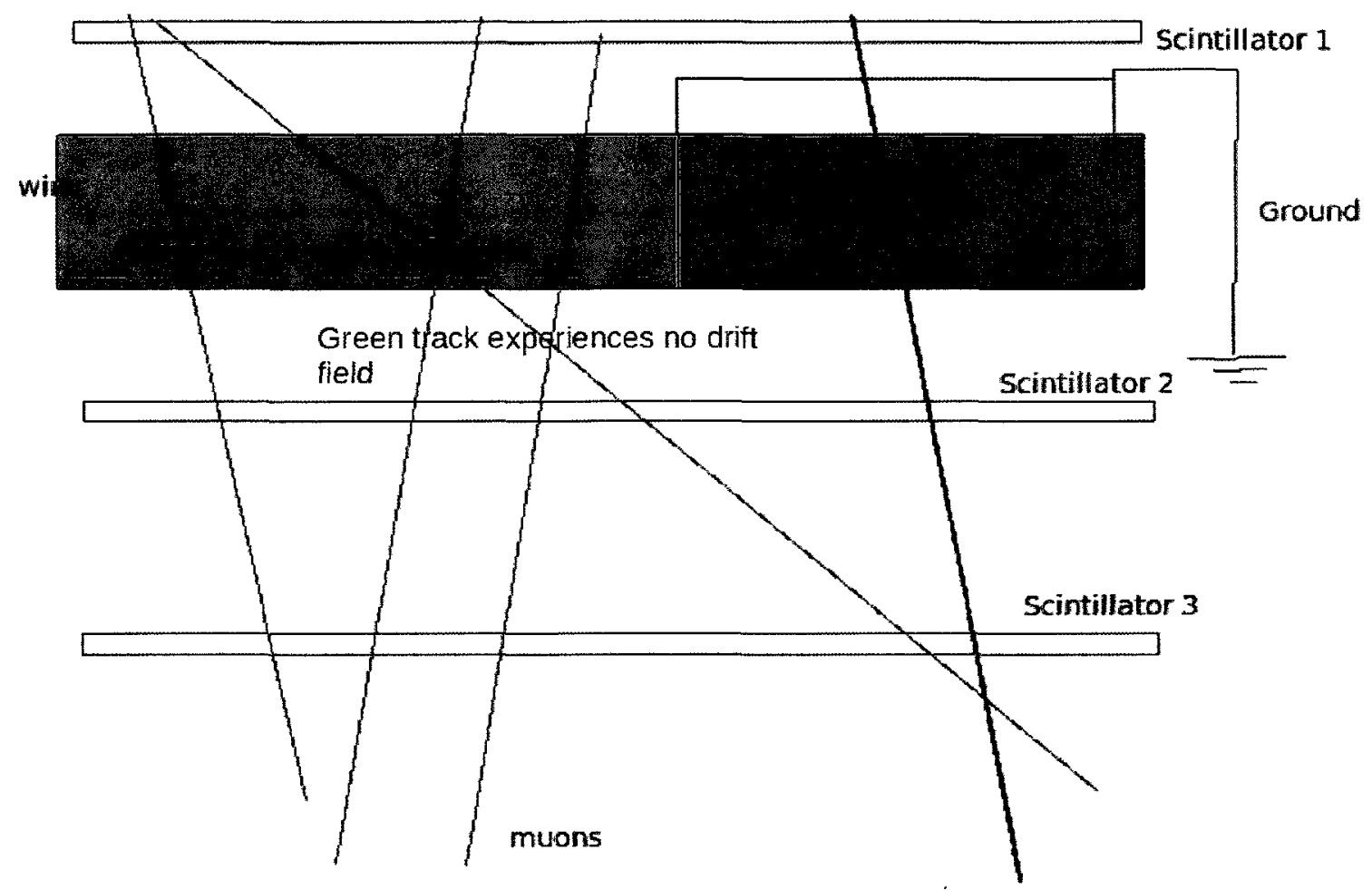

Figure 4.6: Cross-sectional view of the experimental apparatus utilizing the AAR method.

the anode and cathode voltages remains constant for all measurements. In order for this method to be equivalent the constancy of this difference must hold. Moreover, if the difference between the anode and cathode voltages changes the electric field inside of the detector is no longer the same as it would be if no cathode strips were grounded. This can be seen diagrammatically with a similar cross-sectional view as seen in the GD section. This is shown in fig 4.6. In this figure the green region is where there exists no drift field due to a particular strip being grounded. Notice that there are indeed still muons passing through this area and they are interacting with the gas in the detector. However, because there is no electric field present to provide the ionization with extra kinetic energy this ionization is quickly dissipated by recombination with the liberated cations.

This method is not without risk either. One must take extra care when dealing 


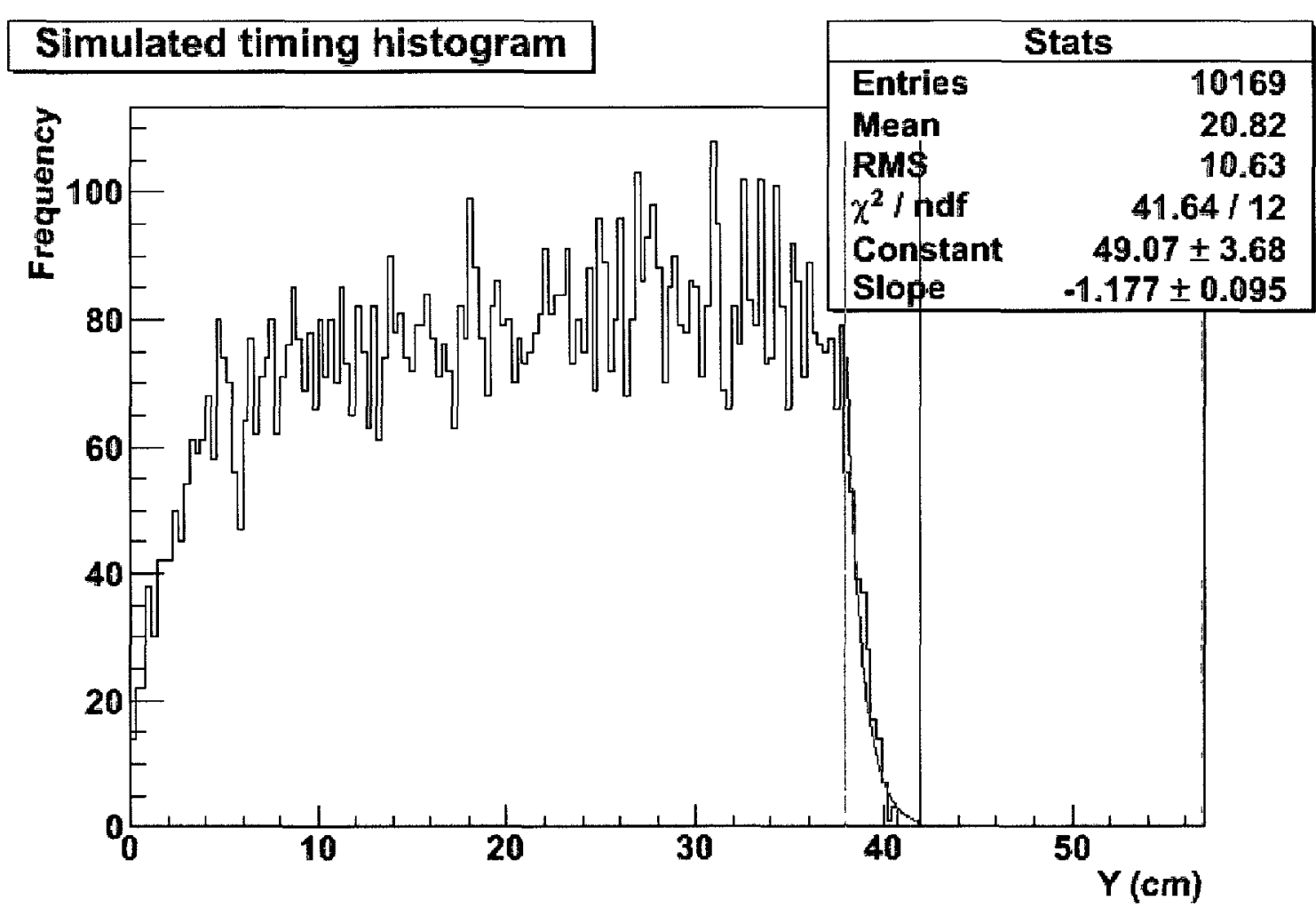

Figure 4.7: A simulated timing distribution for the AAR method. 


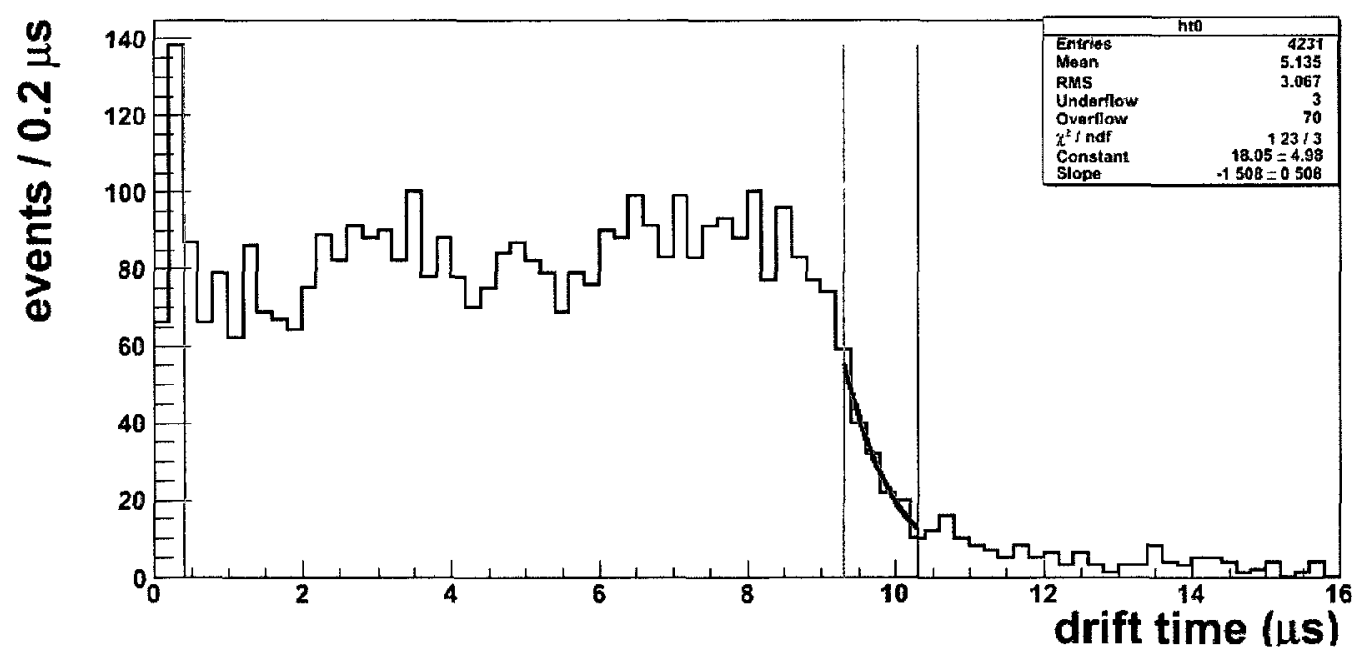

Figure 4.8: An actual timing histogram with fitting and correction to ' $t_{\text {max }}$ ' present from the AAR method.

with delicate HV components. Should a strip's connection become severed it would create a localized distortion in the drift field and this will have negative effects on the measurement of the drift velocity.

As with the GD method it is possible to simulate what the timing distributions will look like. This was done for the AAR method for the sake of preparedness. A simulated timing histogram is shown in fig 4.7. Again, the geometry plays an important role. The cut applied to the timing distribution is not clean. It is gradual over a range of a few $\mu \mathrm{s}$ in the detector. This appears to be best fit with a decaying exponential function. The time at which the exponential fit has been reduced to half of its initial value is taken to be the end of the timing histogram. This is a reasonable approach because unlike in the simulation the real timing distribution will have an amount of background present. The background is mainly due to very wide angled tracks and events that are spuriously correlated to real events by the trigger. The real timing histogram obtained by implementing this method is shown in fig 4.8.

This method employs an algorithm that looks for a large enough difference between bins in order to determine where the shoulder of active area is. An inherent property 
of this method is that it produces an unusually large number of events in a single bin at very small times. This oddity can be accounted for by recognizing it in the algorithm and correcting the time accordingly. The time taken as the 'real' time is $\mathrm{t}_{\text {real }}=\mathrm{t}_{f \imath t}-\mathrm{t}_{\text {strange }}{ }^{1}$. The correction is typically less than $0.5 \mu \mathrm{s}$ but is a serious limiting factor of this method. Another potential drawback to this approach is that having larger statistics don't necessarily improve the accuracy of the fitting. This is because the region being fit is typically a $1 \mu$ s span of time. Given that the drift field is present up to the location of the ground events will be recorded for every arrival time from $\mathrm{t}=0$ to $\mathrm{t}=\mathrm{t}_{\max }$. This statistical inefficiency requires significantly more analysis time simply because there are more events to process. This is an artifact that GD does not suffer from. GD becomes very efficient with roughly $1 / 10^{\text {th }}$ of the statistics that $A A R$ requires.

This chapter will examine some actual data taken with the CRIPT prototypes. As previously mentioned two methods will be examined and one will be chosen to be the primary experimental technique. The data collected for the purposes of this thesis began in January of 2011 and ran until June 2011. Given that charge induction on the 'zig-zag' electrodes has absolutely no bearing on the measurement of drift velocity only necessary components of the apparatus will be considered for the sake of clarity. A photo of the actual CRIPT prototypes in situ in the laboratory is shown in fig 4.9. The data collected in my work were exclusively from detector ${ }^{2} 3$. One detector was used in order to maintain consistency between data at different drift fields and with different gases. The prototypes are fabricated with identical specifications but nevertheless require individual calibrations insofar as drift velocity is concerned. A remedy for this will be presented in a later chapter.

\footnotetext{
${ }^{1} t_{\text {strange }}$ is the time bin corresponding to the anomalously high frequency at low times.

${ }^{2}$ The third detector from the top in fig 4.9
} 


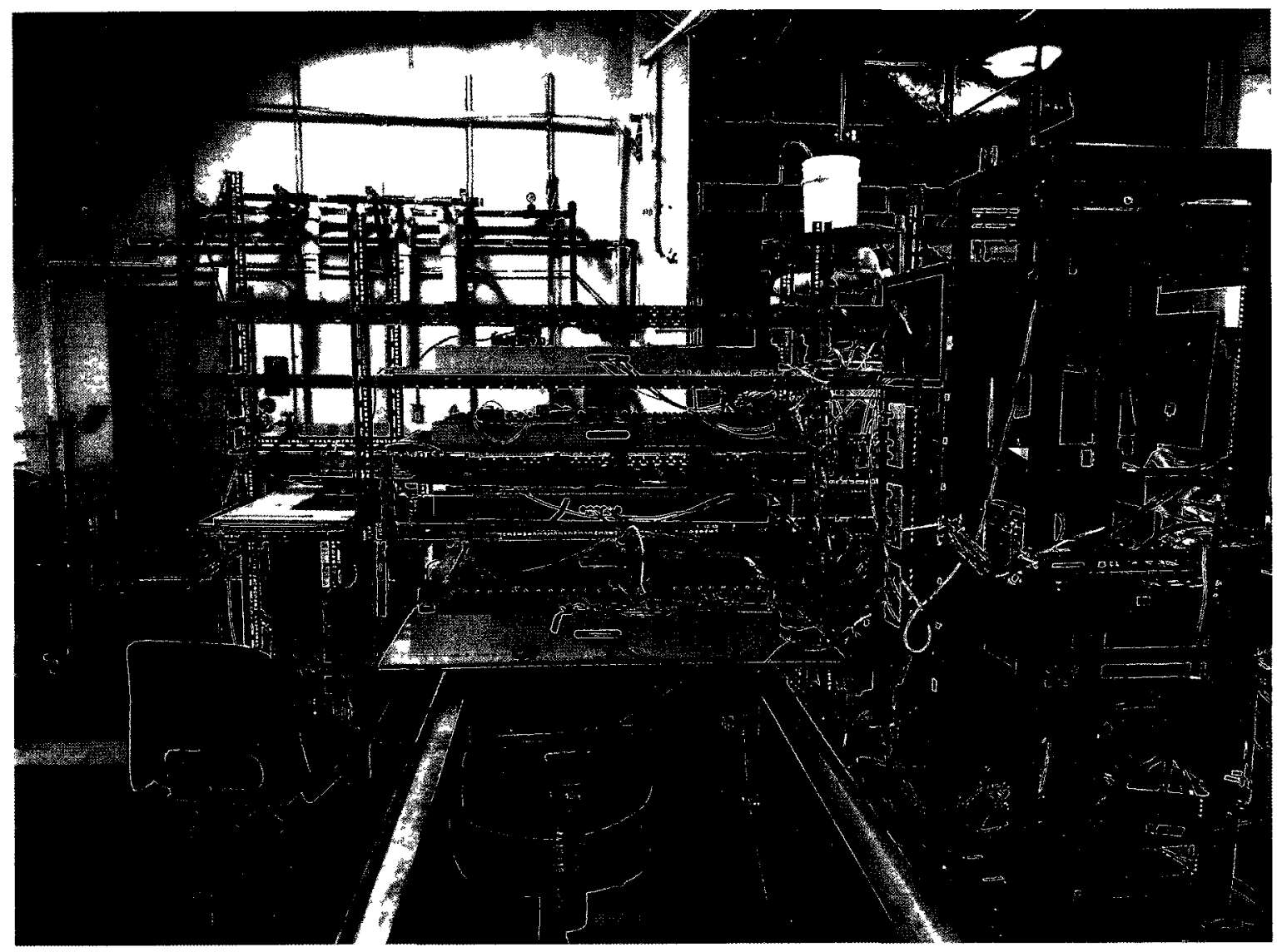

Figure 4.9: The CRIPT drift chamber tracking system in the prototype stage. Shown are the four prototypes. The top three are small scale and bottom is the full sized drift chamber. The drift chambers can be identified by the shielding covering their preamplifier/HV boards. In the photo this appears as the off-white rectangles in the centre of the tower. 


\subsection{AAR vs. GD}

Despite having two appropriate methods at my disposal the purpose of the research was not to investigate measurement techniques. The purpose was to make measurements in a consistent way such that the behaviour of the prototypes can be characterized. It is for that reason the first few results obtained from my work will not only serve the purpose of being results but they will demonstrate the succession of one method over another.

\subsection{1 $\mathrm{Ar}-\mathrm{CH}_{4}$}

This is the first gas used to investigate the CRIPT prototypes. As seen in fig 3.11 argon-methane is the "fastest" gas being tested. That is to say that it possesses the highest drift velocity on the range of electric field strengths experienced in the prototypes. As such it also has the largest amount of both longitudinal and transverse diffusion. Data were collected at both $4000 \mathrm{~V}$ and $6000 \mathrm{~V}$ applied cathode voltage with anode wire voltages of $5820 \mathrm{~V}$ and $7820 \mathrm{~V}$ respectively with both AAR and GD methods and then both directly compared to the simulated results. This is shown in fig 4.10 and fig 4.11 respectively. The linearity of these data series allows for a simple least squares fit to be made. The slope of this fit is the quantity of interest, namely, the drift velocity. The least squares fitting is done with ROOT. This method of characterizing the drift velocity over the entirety of the drift length essentially results in an average velocity.

$$
t=\frac{\Delta V}{\langle V\rangle}=\frac{\left|v_{M C C}-v_{\text {exp }}\right|}{\frac{\left|v_{M C C}\right|+\left|v_{e x p}\right|}{2}}
$$

It can be seen immediately that one experimental method seems to be in better agreement than the other. The appropriate way to quantize the agreement is to perform a consistency test. This is shown in eqn 4.1. Here $\mathrm{v}_{M C}$ is the slope of 


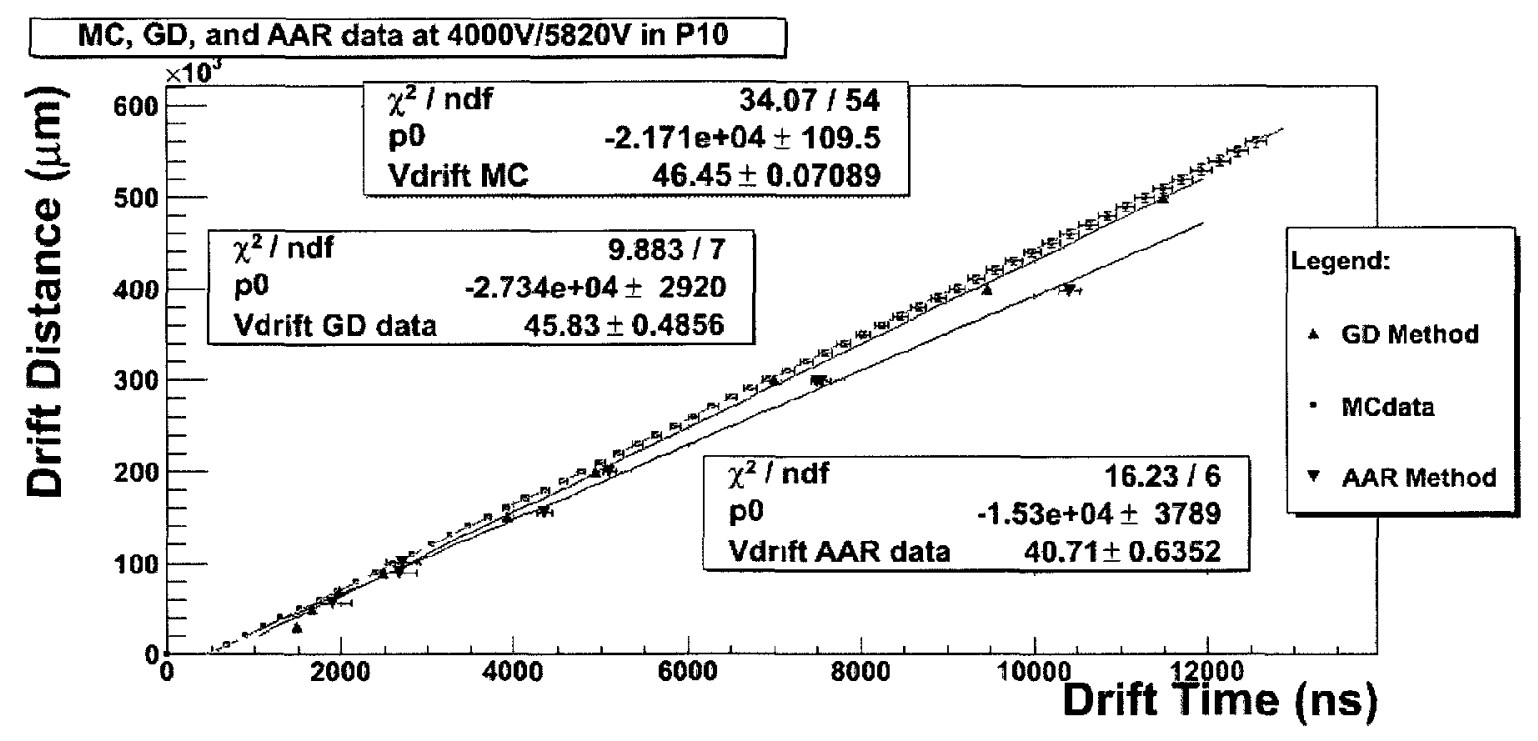

Figure 4.10: GD and AAR data plotted linearly along with the Monte Carlo for $4000 \mathrm{~V}$ applied to the cathode and $5820 \mathrm{~V}$ applied to the anode. The error bars on the GD data points are too small to be seen. Note: P10 is the common name for $\mathrm{Ar}-\mathrm{CH}_{4}$.

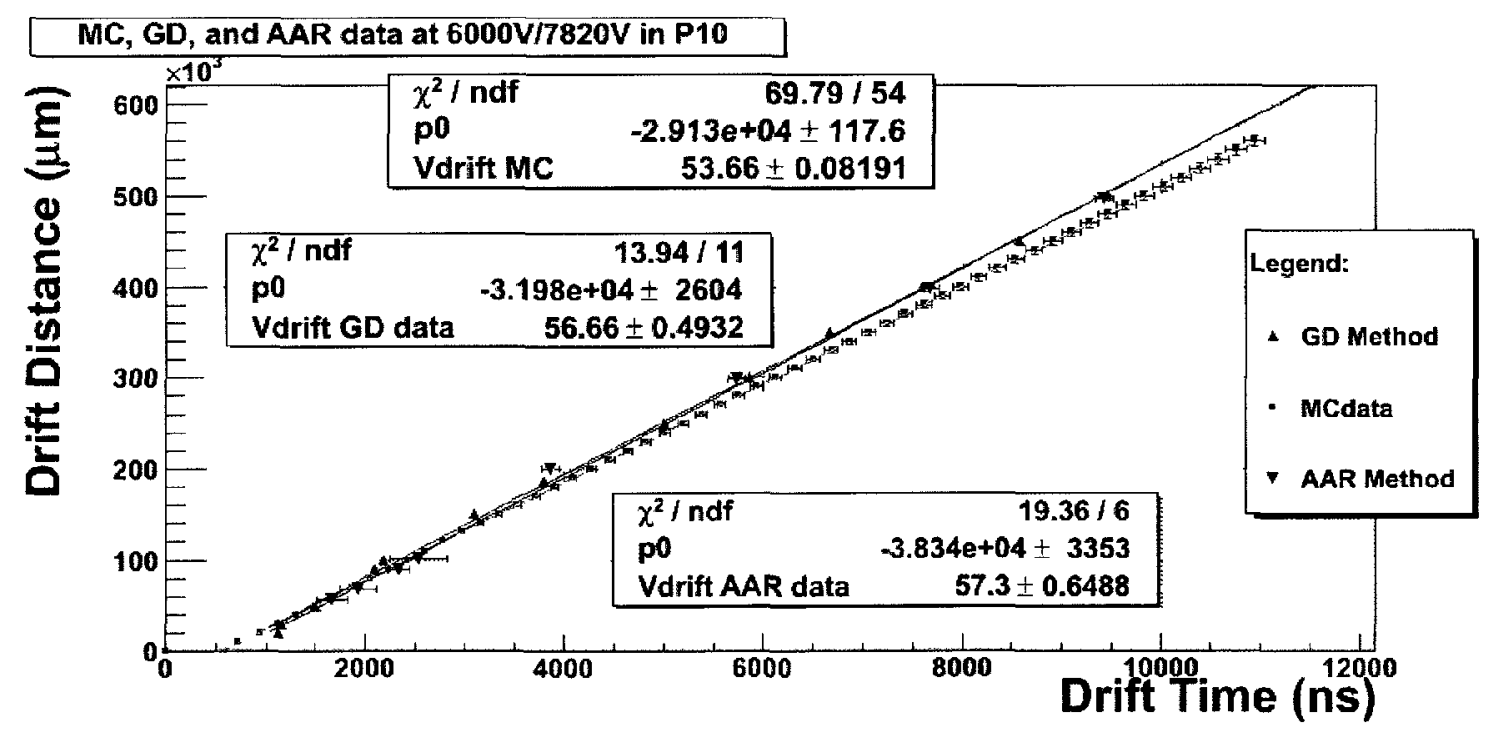

Figure 4.11: GD and AAR data plotted linearly along with the Monte Carlo for $6000 \mathrm{~V}$ applied to the cathode and $7820 \mathrm{~V}$ applied to the cathode. 
the Monte Carlo data, $\mathrm{v}_{\text {exp }}$ is the slope of the method in question, and ' $\mathrm{t}$ ' is a pure number ${ }^{3}$. Ideally t would be equal to zero for perfect agreement between experiment and theory. This magnitude of agreement is how the results will be compared to simulation. The results of fig 4.10 and fig 4.11 are summarized in table 4.1.

\begin{tabular}{cccccc}
\hline \hline Cathode Voltage & MC velocity & GD velocity & AAR velocity & $\mathrm{t}_{G D}$ & $\mathrm{t}_{A A R}$ \\
\hline $4000 \mathrm{~V}$ & $46.45 \pm 0.07$ & $45.94 \pm 0.49$ & $40.71 \pm 0.64$ & 0.011 & 0.1317 \\
$6000 \mathrm{~V}$ & $53.66 \pm 0.08$ & $56.66 \pm 0.49$ & $57.3 \pm 0.65$ & 0.0544 & 0.0656 \\
\hline \hline
\end{tabular}

Table 4.1: Summary of both simulated and experimental data in $\mathrm{Ar}-\mathrm{CH}_{4}$. The units of drift velocity are $\mu \mathrm{m} / \mathrm{ns}$.

It is important to note not only how the experimental techniques compare to the predictions from the simulations but also how consistent they are with themselves. That is to say how well they are fit by a line. The reduced chi-squared, denoted by $\chi_{r e d}^{2}$, is a measure of the quality of the fitting. The results of this statistical test are shown in table 4.2. This clearly shows that the GD method is much more self consistent. This stems from the nature of the error associated with this method which will be described in a later section.

\begin{tabular}{cccc}
\hline \hline Cathode Voltage & $\mathrm{MC} \chi_{\text {red }}^{2}$ & $\mathrm{GD} \chi_{\text {red }}^{2}$ & $\mathrm{AAR} \chi_{\text {red }}^{2}$ \\
\hline $4000 \mathrm{~V}$ & 0.63 & 1.41 & 2.71 \\
$6000 \mathrm{~V}$ & 1.29 & 1.26 & 3.22 \\
\hline \hline
\end{tabular}

Table 4.2: Summary of $\chi_{\text {red }}^{2}$ for P10 gas.

\footnotetext{
${ }^{3}$ Subscripts applied to ' $t$ ' refer to the method being compared to the Monte Carlo
} 


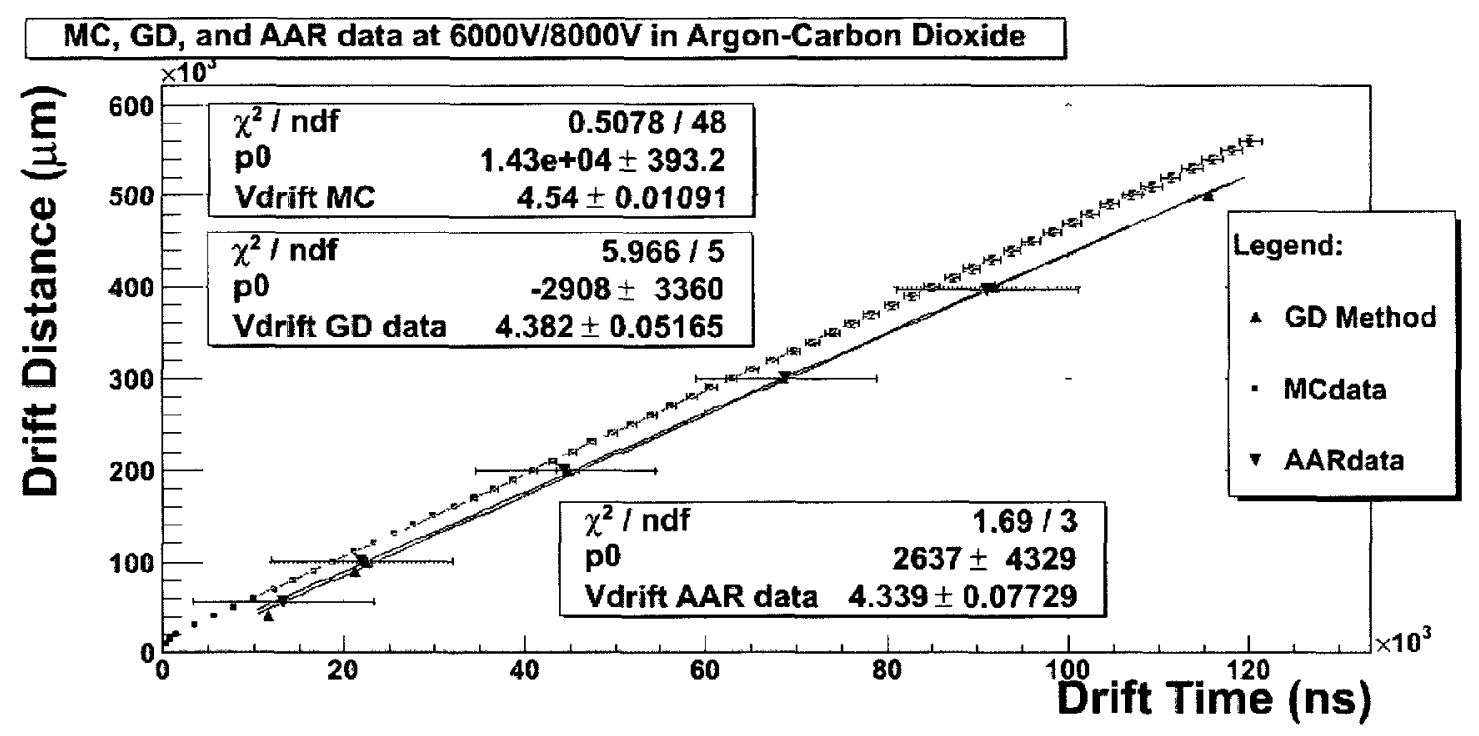

Figure 4.12: GD and AAR data plotted linearly along with the Monte Carlo for $6000 \mathrm{~V}$ applied to the cathode and $8000 \mathrm{~V}$ applied to the anode.

\subsection{2 $\mathrm{Ar}-\mathrm{CO}_{2}$}

Argon-Carbon Dioxide is considered to be a slow gas. This means its velocity is low along with both diffusion values. Due to its exceptionally low velocity this gas presented a problem with the CRIPT prototypes. The low velocity made it much more likely that multiple events would be detected in a very small time window. This is an effect called 'event pileup' and it is the reason that no data were recorded for this gas at a cathode voltage of $4000 \mathrm{~V}$. This is not all bad news. It gives a clear lower limit on the drift velocity in these prototypes. As seen in fig 3.17 there is only a very slight difference between the simulated velocities of $\mathrm{Ar}-\mathrm{CO}_{2}$ in the detectors and as will be shown meaningful data were relatively easily obtained for this gas at a cathode voltage of $6000 \mathrm{~V}$. One important note is that because the ionization will drift much more slowly it was necessary to broaden the DAQ sampling window by a factor of 10 .

Fig 4.12 shows the linear plots for the $6000 \mathrm{~V}$ data series for both experimental methods and the Monte Carlo results. Applying the same analysis techniques as 


\begin{tabular}{cccccc}
\hline \hline Cathode Voltage & MC velocity & GD velocity & AAR velocity & $\mathrm{t}_{G D}$ & $\mathrm{t}_{A A R}$ \\
\hline $6000 \mathrm{~V}$ & $4.639 \pm 0.007$ & $4.382 \pm 0.051$ & $4.339 \pm 0.077$ & 0.0569 & 0.0668 \\
\hline \hline
\end{tabular}

Table 4.3: Summary of both simulated and experimental data in $\mathrm{Ar}-\mathrm{CO}_{2}$. The units of drift velocity are $\mu \mathrm{m} / \mathrm{ns}$.

\begin{tabular}{cccc}
\hline \hline Cathode Voltage & $\mathrm{MC} \chi_{\text {red }}^{2}$ & $\mathrm{GD} \chi_{\text {red }}^{2}$ & $\mathrm{AAR} \chi_{\text {red }}^{2}$ \\
\hline $6000 \mathrm{~V}$ & 0.0106 & 1.19 & 0.56 \\
\hline \hline
\end{tabular}

Table 4.4: Summary of $\chi_{\text {red }}^{2}$ for $\mathrm{Ar}-\mathrm{CO}_{2}$ gas.

before results in tables 4.3 and 4.4 respectively.

\subsection{3 $\quad$ Ar- $\mathrm{C}_{2} \mathrm{H}_{6}$}

This gas is considered to be a moderately fast gas. As such it was chosen to be examined with both techniques simultaneously in order to understand how well a data series can be fit when both methods are used together. What is meant by used together is not that the raw data was acquired implementing both techniques at once but rather some data points were taken with one method and some with the other and then all results plotted together on the same plots. The results of the linear fit for both cathode voltages are shown in figures 4.13 and 4.14 respectively. Finally, the results are summarized in tables 4.5 and 4.6 respectively.

Despite the relative difference between the measured values of the drift velocity being small for both drift fields examined here it must be noted the exceptionally poor reduced chi-squared values from the linear fits. This implies that the two methods are not consistent with respect to each other given that all previous values of chi-squared - when the methods were applied individually - are much more reasonable. 


\begin{tabular}{cccc}
\hline Cathode Voltage & MC velocity & AAR/GD velocity & $\mathrm{t}_{\text {GD/AAR }}$ \\
\hline $4000 \mathrm{~V}$ & $33.34 \pm 0.10$ & $31.26 \pm 0.23$ & 0.0644 \\
$6000 \mathrm{~V}$ & $39.63 \pm 0.17$ & $37.55 \pm 0.33$ & 0.0538 \\
\hline
\end{tabular}

Table 4.5: Summary of both simulated and experimental data in $\mathrm{Ar}-\mathrm{C}_{2} \mathrm{H}_{6}$. The units of drift velocity are $\mu \mathrm{m} / \mathrm{ns}$.

\begin{tabular}{ccc}
\hline Cathode Voltage & MC $\chi_{\text {red }}^{2}$ & AAR/GD $\chi_{\text {red }}^{2}$ \\
\hline $4000 \mathrm{~V}$ & 0.314 & 14.54 \\
$6000 \mathrm{~V}$ & 0.7 & 7.12 \\
\hline \hline
\end{tabular}

Table 4.6: Summary of $\chi_{\text {red }}^{2}$ for $\mathrm{Ar}-\mathrm{C}_{2} \mathrm{H}_{6}$ gas.

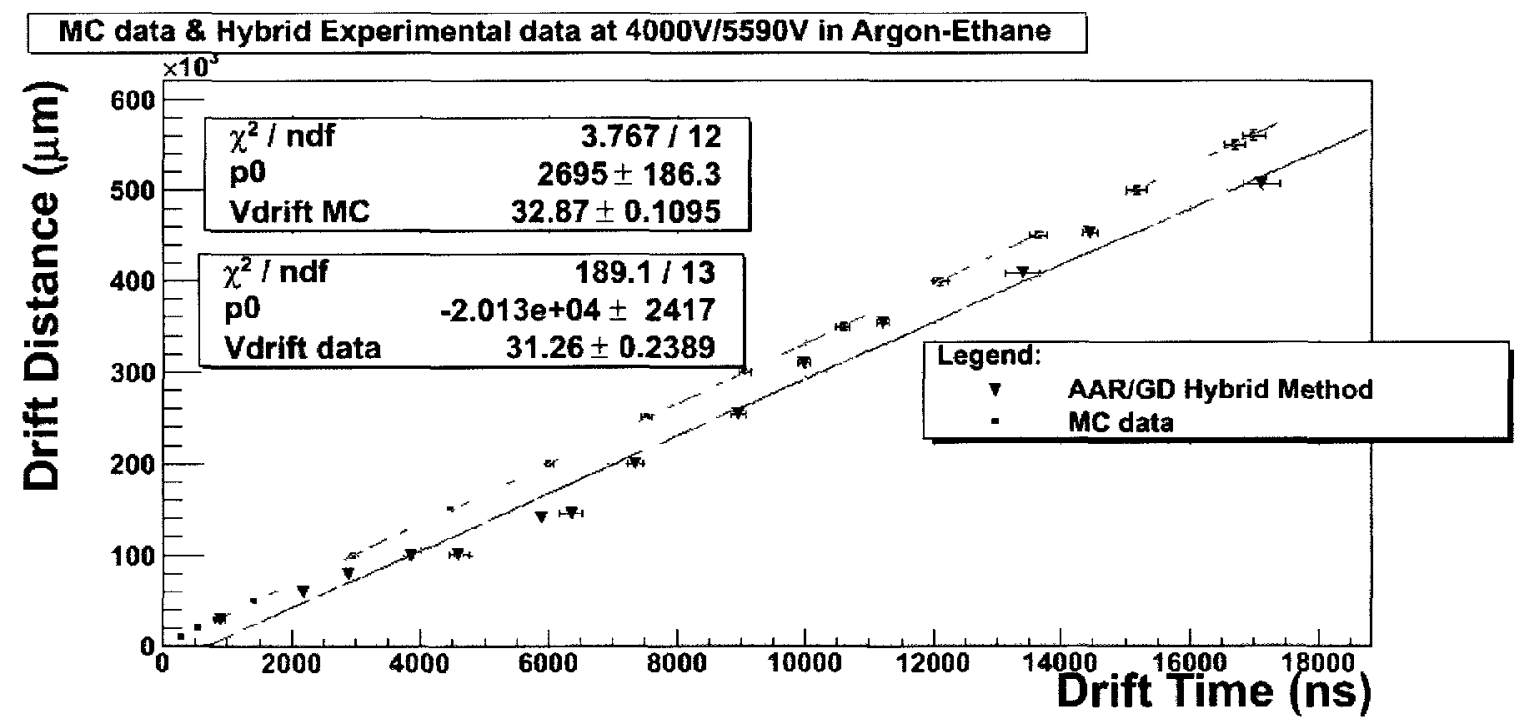

Figure 4.13: GD and AAR hybrid data plotted linearly along with the Monte Carlo for $4000 \mathrm{~V}$ applied to the cathode and $5590 \mathrm{~V}$ applied to the anode. 


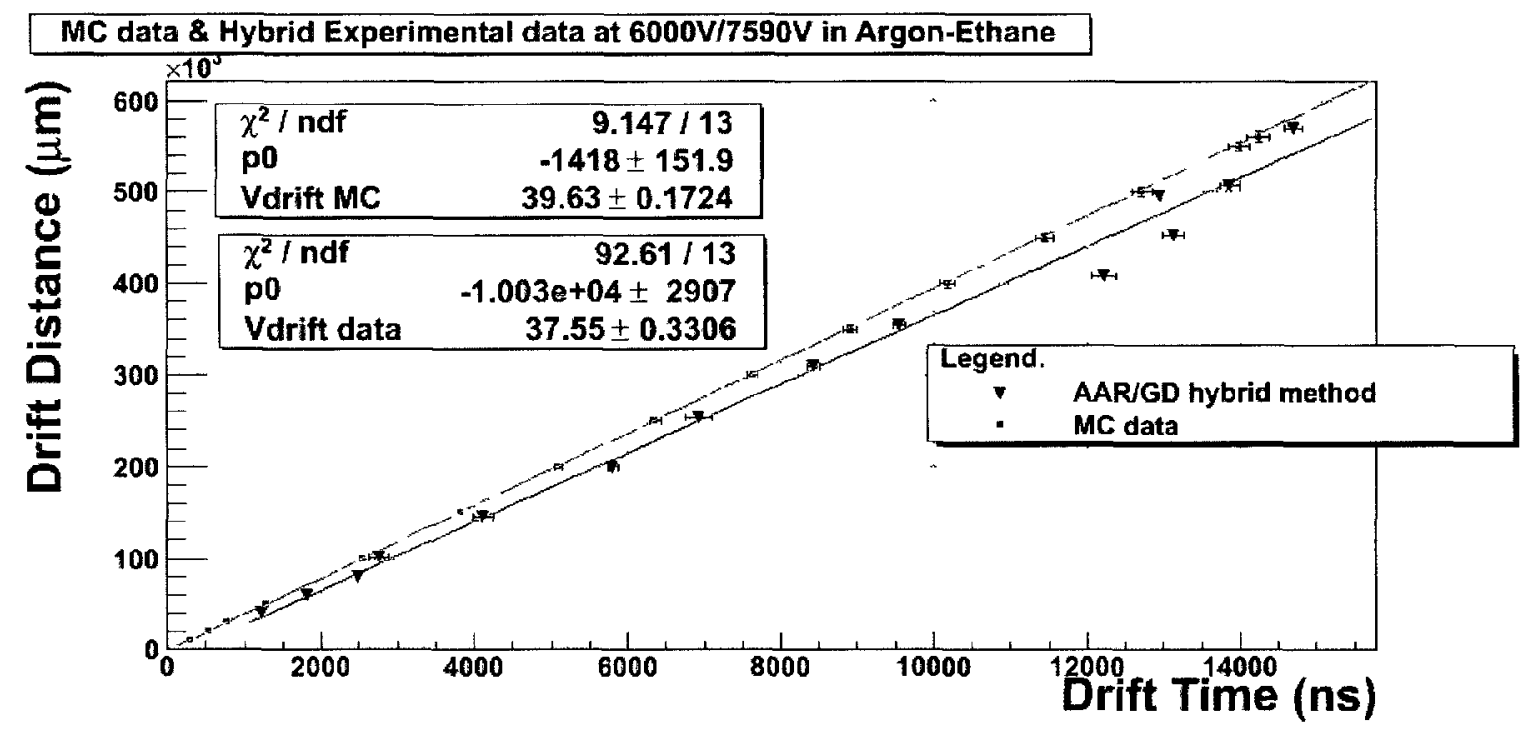

Figure 4.14: GD and AAR hybrid data plotted linearly along with the Monte Carlo for $6000 \mathrm{~V}$ applied to the cathode and $7590 \mathrm{~V}$ applied to the anode.

\subsubsection{Choice of method}

Given the results presented in the subsections above a conclusion can be drawn. The most appropriate choice for the primary method of data collection should be geometrical discrimination. It consistently produces the smallest relative differences between experiment and theory and it consistently produces the best quality of fits when fitting with a line. It has been shown that combining the two methods is not a viable solution as quality of the fit greatly suffers from this. This is due to each method having different systematic errors. This in turn allows for greater variation between the distribution of data points and thusly affects the reduced chi-squared values. The uncertainty of AAR is a function of the arbitrary choice of bin size in the timing histograms. In order to fit the exponential decay even reasonably well a range of bin sizes must be examined. The GD method does not suffer from this drawback as the range of bin sizes that produce a Gaussian is large.

The statistics are not the only necessary rationale in deciding to proceed with GD. GD is also a more efficient method than AAR. This inherent difference stems directly 
from the nature of each approach. GD attenuates all but necessary information in the detector whereas AAR does not. Consider the number of necessary events that are required before the timing histogram begins to take a discernible shape. It has been shown that roughly 2000 muon events per GD data point will produce the Gaussian distribution very effectively. AAR on the other hand requires the continuum of the histogram - up to the region being examined - to be filled with statistics. The number of events in AAR timing distributions is by nature much larger than GD. Moreover, simply taking a larger number of muon events does not necessarily imply that improvement will be found in the region where the exponential decay function is being fit. The events will happen randomly throughout the drift length up to where the field no longer exists. One must also consider the time required to perform analysis to extract timing information from the raw binary data files. Simply speaking the more events there are the longer it will take to process all of them.

The resolution of each method is also different. What is meant here by resolution is not how accurately the drift velocity can be determined so much as the direct limitation of possible position measurements. That is to say that AAR is limited to a maximum number of data points. This number is less than the number of cathode strips between the 'zig-zag' and the aluminum side rails. Moreover, since the electrode size is also fixed, the smallest difference between positions is fixed. GD on the other hand is highly adaptable. The size of the overlapping scintillator area can be varied, its position from the anode plane can be varied, and the only limiting factor in how many positions can be measured is the tool with which one measures the position. More accurate position measurements mean more data points can be taken and therefore a better estimation of the drift velocity can be made.

It is for these reasons that all subsequent data utilizes the GD method unless explicitly stated otherwise. 


\section{Chapter 5}

\section{Results}

As seen in the chapter concerning the experimental techniques used in this research some interesting results were obtained. Generally speaking, both methods were able to acquire data that were in very good agreement with the Monte Carlo simulation. The techniques used to analyze the raw data involve two main types of plots. The first is the linear plot of drift distance verses drift time and the second is drift velocity verses drift distance. They are both useful for their own reasons. The former allows for an average velocity to be found with little statistical error while the other provides a glimpse of how the velocities are distributed along the length of drift. The $v_{\text {drift }}$ vs. distance plots are an effective way to characterize the detectors. This is the case because the general objective of the prototypes is to examine their suitability for tracking muons. The more accurately the velocity is known throughout the detector the more accurately the coordinate perpendicular to the wire may be reconstructed.

The following sections will present the results obtained from my work with the CRIPT small scale prototype drift chamber. Results presented in the previous chapter will not be restated explicitly but will appear in summary tables as necessary.

The results for each gas will be presented in individual sections in this chapter with the applied cathode voltages being the subsections. Results will be summarized as they are presented. The reason for using the applied cathode voltages to discriminate 
between trials of gases is that the magnitudes of the voltages are simply more elegant than their corresponding drift fields. Cathode voltages of $3 \mathrm{kV}, 4 \mathrm{kV}$, and $6 \mathrm{kV}$ correspond to $52.6 \mathrm{~V} / \mathrm{cm}, 70.2 \mathrm{~V} / \mathrm{cm}$, and $105.2 \mathrm{~V} / \mathrm{cm}$ drift fields respectively given that the maximum drift length is $57 \mathrm{~cm}$. As seen in eqn 3.1 the calculation of the field is a very simple exercise.

\section{$5.1 \quad \mathrm{Ar}_{-} \mathrm{CH}_{4}$}

\section{$5.1 .13000 \mathrm{~V}$}

\begin{tabular}{cccc}
\hline \hline Cathode Voltage & MC velocity & Experimental velocity & $\mathrm{t}_{\exp }$ \\
\hline $3000 \mathrm{~V}$ & $38.12 \pm 0.17$ & $19.27 \pm 0.27$ & 0.6594 \\
\hline \hline
\end{tabular}

Table 5.1: Summary of experimental results for P10 at $3000 \mathrm{~V}$ on the cathode. The units of drift velocity are $\mu \mathrm{m} / \mathrm{ns}$.

\begin{tabular}{ccc}
\hline \hline Cathode Voltage & MC $\chi_{\text {red }}^{2}$ & Experimental $\chi_{\text {red }}^{2}$ \\
\hline $3000 \mathrm{~V}$ & 0.00555 & 6.28 \\
\hline \hline
\end{tabular}

Table 5.2: Summary of $\chi_{\text {red }}^{2}$ for $\mathrm{Ar}-\mathrm{CH}_{4}$ gas.

The $v_{d r i f t}$ vs. $\mathrm{d} \operatorname{plot}^{1}$ for $3000 \mathrm{~V}$ on the cathode and $4520 \mathrm{~V}$ on the anode is shown in fig 5.1. Immediately it is obvious that there is poor agreement between the experimental results and the simulation. The standard deviation of the experimental results is $1.63 \mu \mathrm{m} / \mathrm{ns}$ and the mean of the spread is $19.52 \mu \mathrm{m} / \mathrm{ns}$. It should be noted that this data series was taken between May 2011 and June 2011. This significance will be explained in greater detail later.

\footnotetext{
${ }^{1}$ 'd' is for distance.
} 


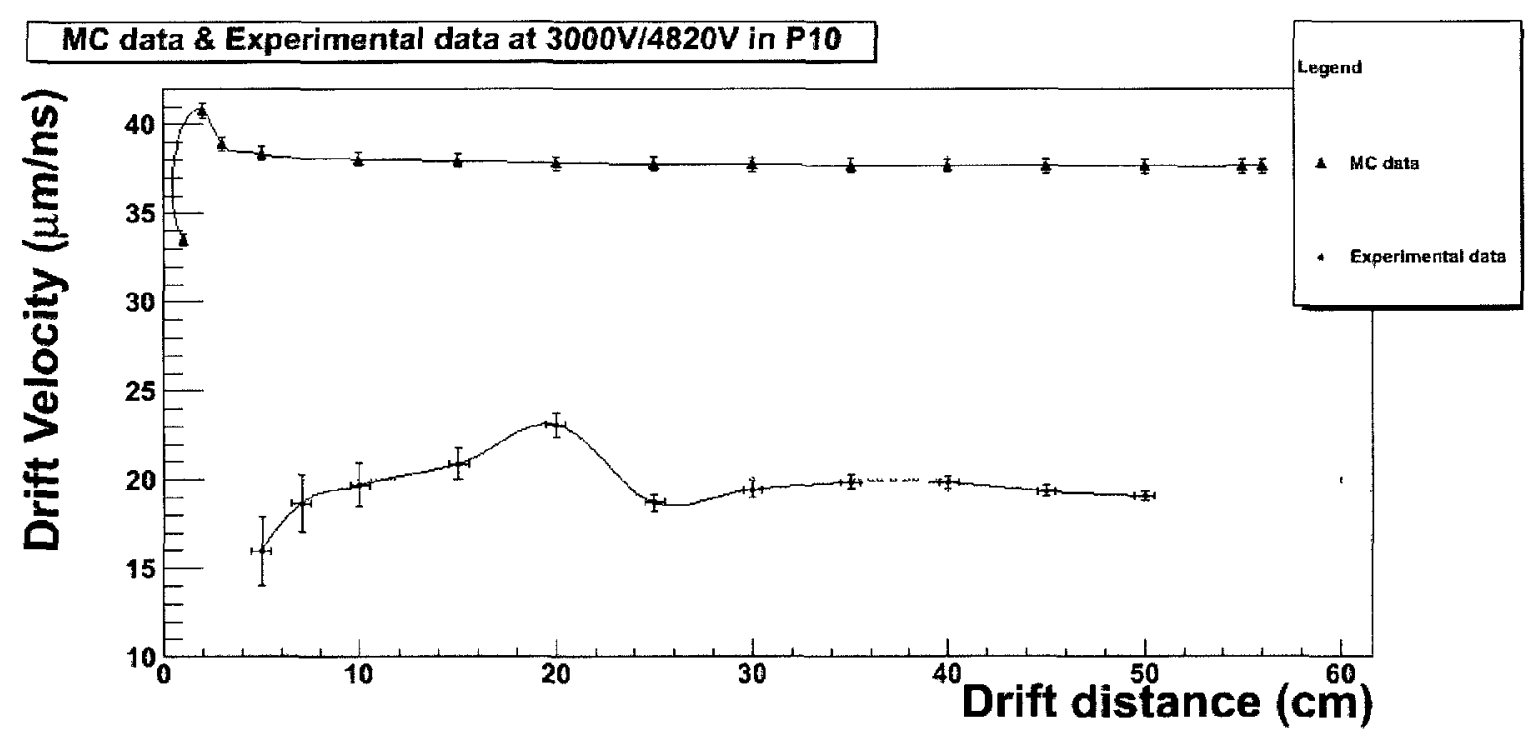

Figure 5.1: Drift velocity curve for $3000 \mathrm{~V}$. These data were likely taken after contamination occurred.

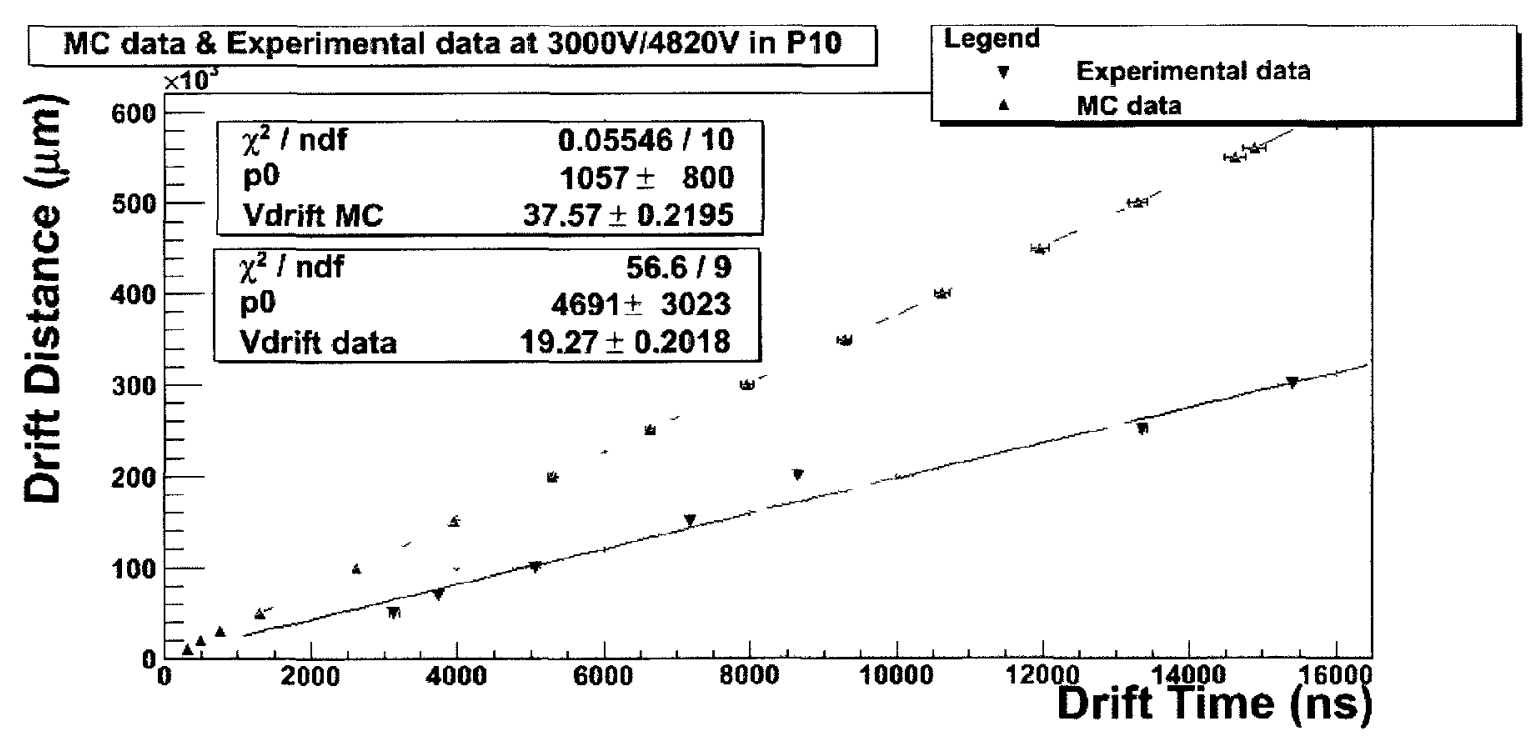

Figure 5.2: Linear plot for $3000 \mathrm{~V}$. Note: Error bars on the experimental data are too small to be seen. These data were likely taken after contamination occurred. 


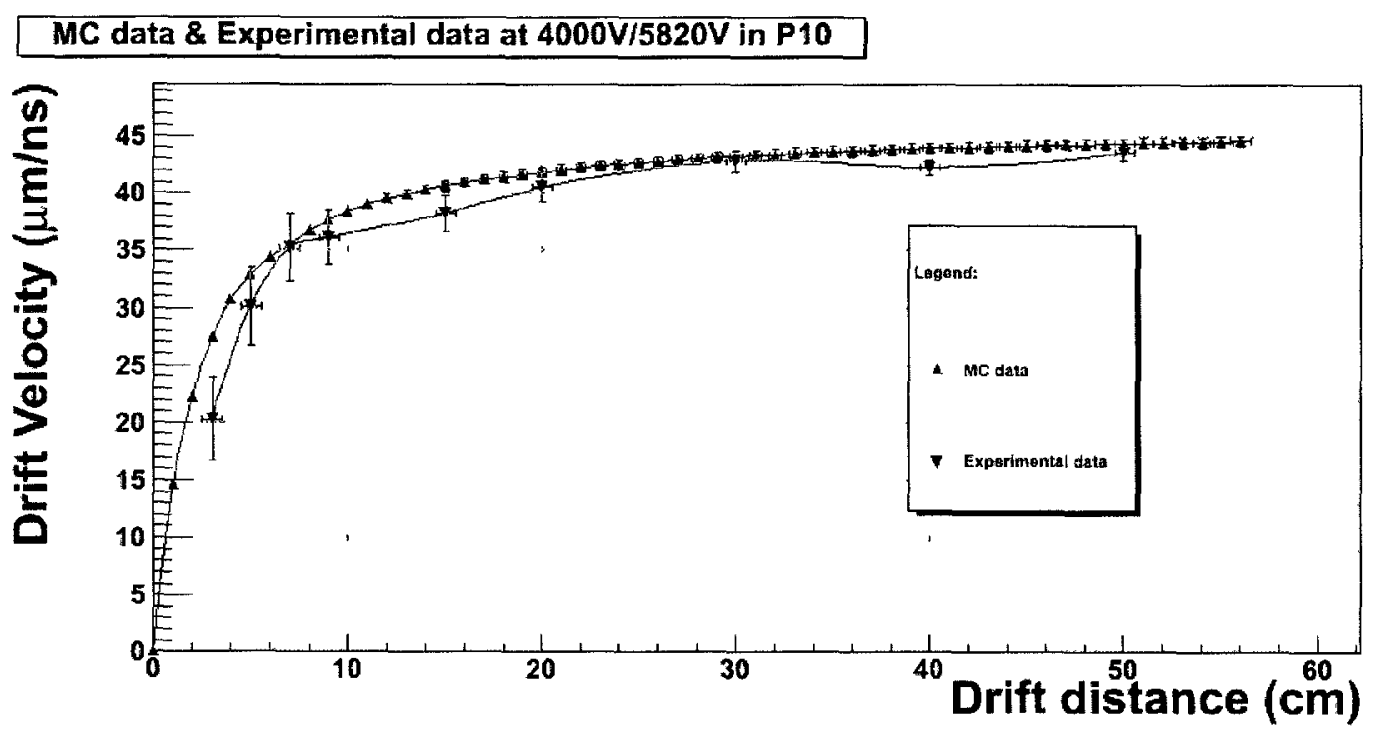

Figure 5.3: Drift velocity curve for $4000 \mathrm{~V}$.

Fig 5.2 shows the linear plot of experimental results and simulation. The average velocity from the linear plot is very similar to the mean velocity from the curve. The main difference is that the error associated with the the mean value taken from the curve is the standard deviation whereas the error on the linear fit is a result of the least squares fit and it consequently much smaller.

\section{$5.1 .24000 \mathrm{~V}$}

As previously seen there was good agreement between the measurements and the simulations for this gas at this particular drift field. Fig 5.3 shows the drift velocity over the range of drift distances. It is not meaningful to use the standard deviation of the data in the curve for comparative reasons because the nature of the gas allows for a large variation over the drift length. 


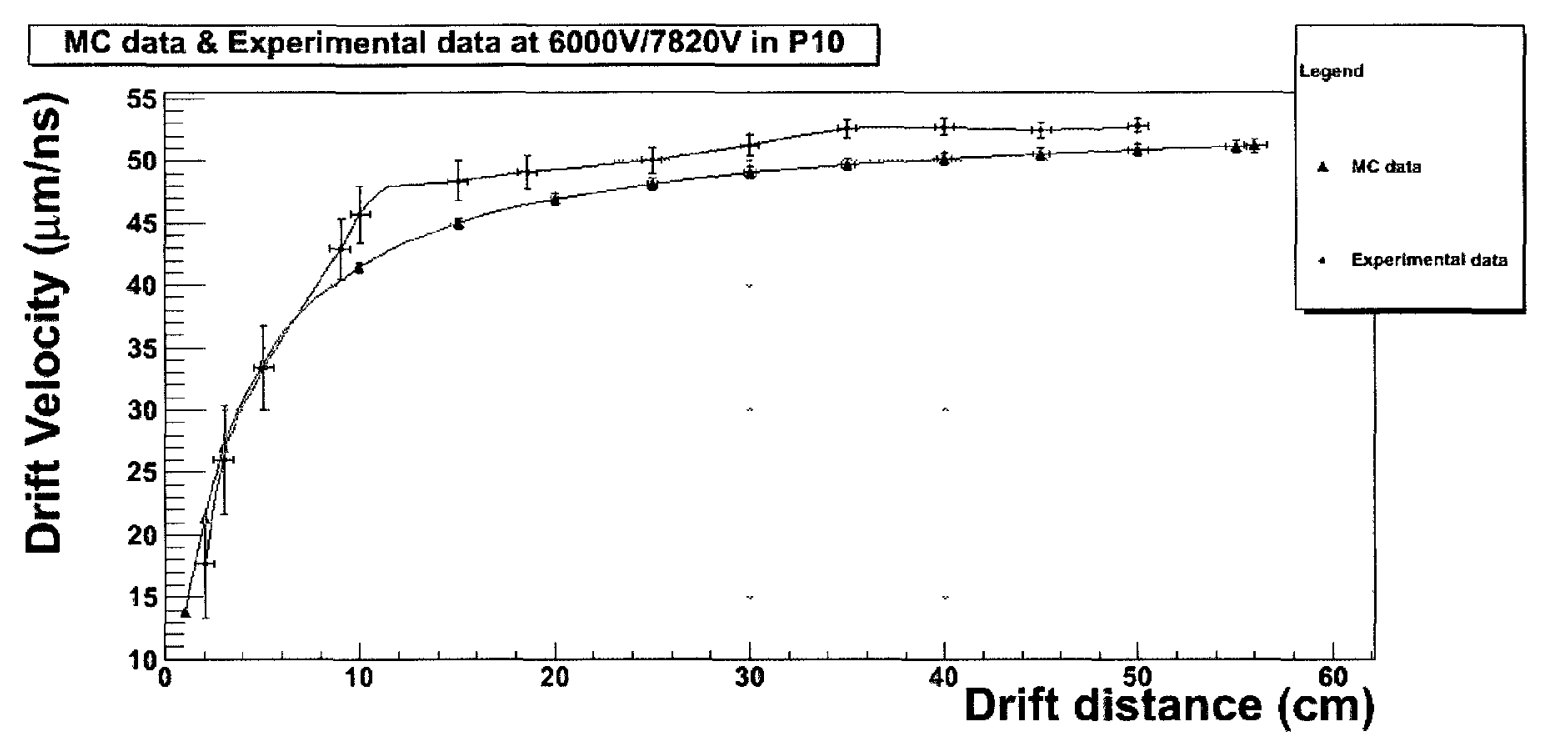

Figure 5.4: Drift velocity curve for $6000 \mathrm{~V}$.

\subsection{3 $6000 \mathrm{~V}$}

Again, the linear plots have been shown in a previous section. The drift velocity curve is shown in fig 5.4. One very interesting feature of this curve is that the experimental results are, for the most part, above the simulation results. This is interesting because the simulation does not account for diffusion whatsoever whereas the experimental data has undergone diffusion as the ionization was transported to the anode. This can most easily be explained by the fact that the curve shown at the bottom of fig 3.11 which relates the drift velocity to the electric field reaches a maximum and begins to decrease. Fig 5.5 shows the behaviour of ionization in P10 over a larger range of electric fields. It can be seen that slightly below $200 \mathrm{~V} / \mathrm{cm}$ the drift velocity reaches a peak. In the CRIPT drift chamber simulation ionization electrons can experience fields in excess of $30 \mathrm{kV} / \mathrm{cm}$ in the region very close to the anode wire. It is likely that this is simply an inherent property of P10 gas. 

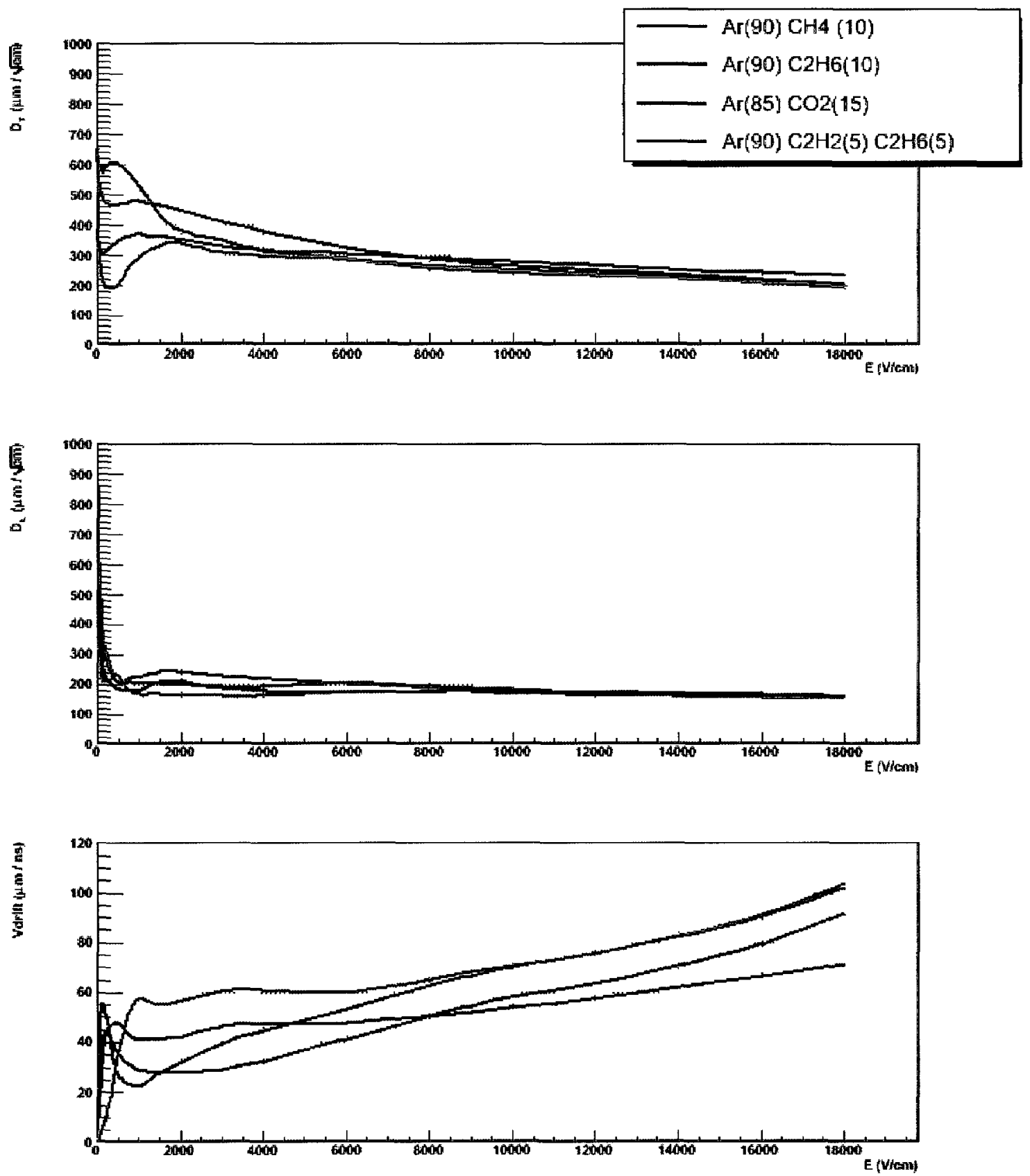

Figure 5.5: MAGBOLTZ results for all gases used in this research shown for a larger range of electric field strengths The drift velocity is shown on the bottom plot 


\section{$5.2 \quad \mathrm{Ar}-\mathrm{C}_{2} \mathrm{H}_{6}$}

\subsection{1 $4000 \mathrm{~V}$}

Argon-ethane is the next fastest gas compared to P10 that was examined in the CRIPT prototypes. As previously seen the average drift velocities are stated in table 4.5. Fig 5.6 shows the drift velocity over the drift range. The mean value from this plot, since no real shape is evident other than a spread, is $28.62 \mu \mathrm{m} / \mathrm{ns}$ with a standard deviation of $2.97 \mu \mathrm{m} / \mathrm{ns}$. One important factor for this plot is that it was made with both AAR and GD methods. This is why the error bars are large at small drift distances. The difference between the error on AAR data and GD data will be covered later in this chapter. It can be seen that there is consistency between the two methods here and that the results, on average, agree with the simulation. The positions of $3 \mathrm{~cm}, 6 \mathrm{~cm}, 8 \mathrm{~cm}, 10 \mathrm{~cm}, 14 \mathrm{~cm}$, and $57 \mathrm{~cm}$ were taken with the GD method while the rest are AAR. A very interesting point of comparison is the data near $14 \mathrm{~cm}$. The two positions are $14 \mathrm{~cm}$ and $14.45 \mathrm{~cm}$ for GD and AAR respectively and they are both within each others' errors. There is $1.8 \sigma$ agreement between the experimental data and the simulation. 


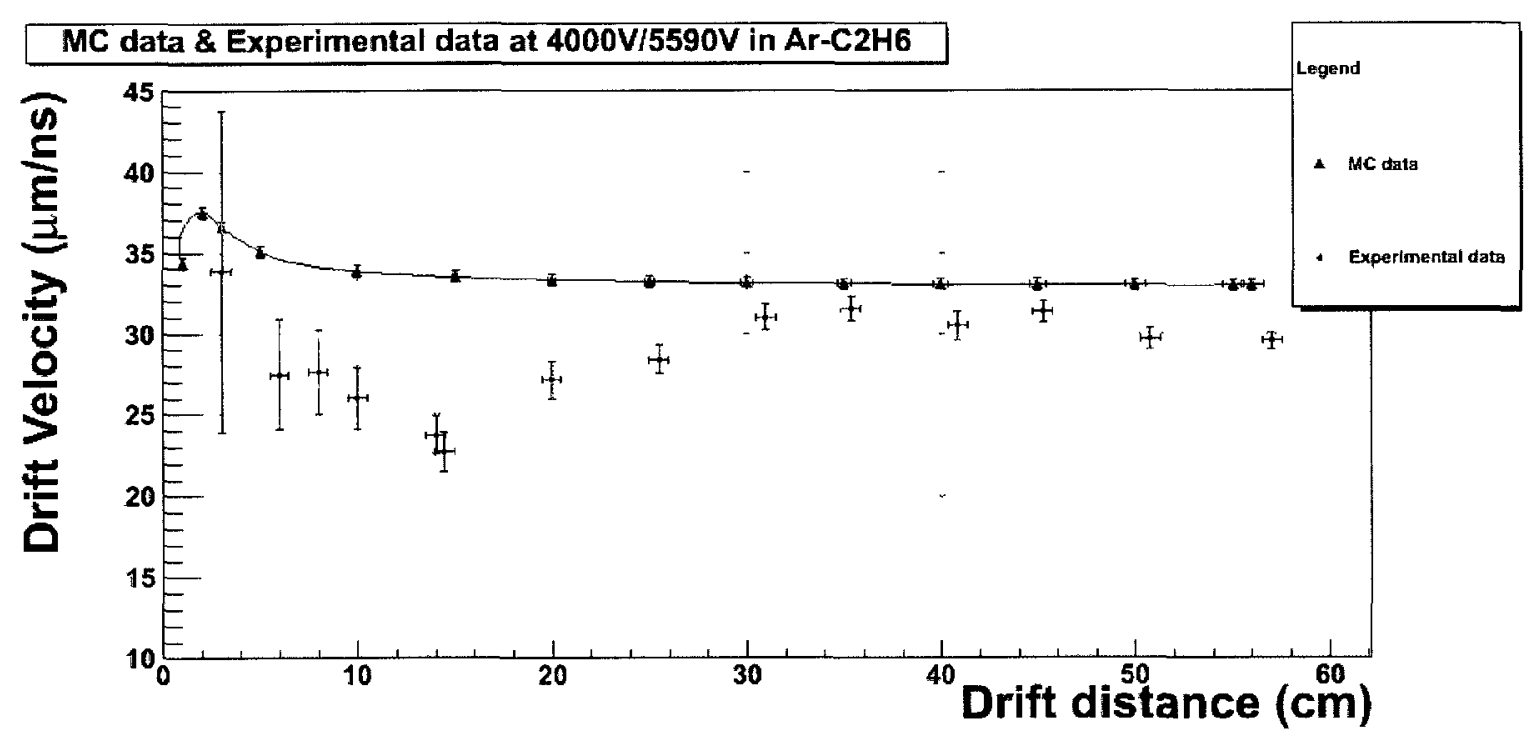

Figure 5.6: Drift velocity curve for $4000 \mathrm{~V}$

\section{$5.2 .26000 \mathrm{~V}$}

Again, the previous results are summarized in table 4.5. A similar approach to the $4000 \mathrm{~V}$ data was taken here. Data points at $4 \mathrm{~cm}, 6 \mathrm{~cm}$, and $8 \mathrm{~cm}$ were taken with the GD method. Fig 5.7 shows the results. The mean velocity from this curve is $35.35 \mu \mathrm{m} / \mathrm{ns}$ with a standard deviation of $1.98 \mu \mathrm{m} / \mathrm{ns}$. This implies that there is $1.8 \sigma$ agreement here as well. These data were taken between February 2011 and March 2011. 


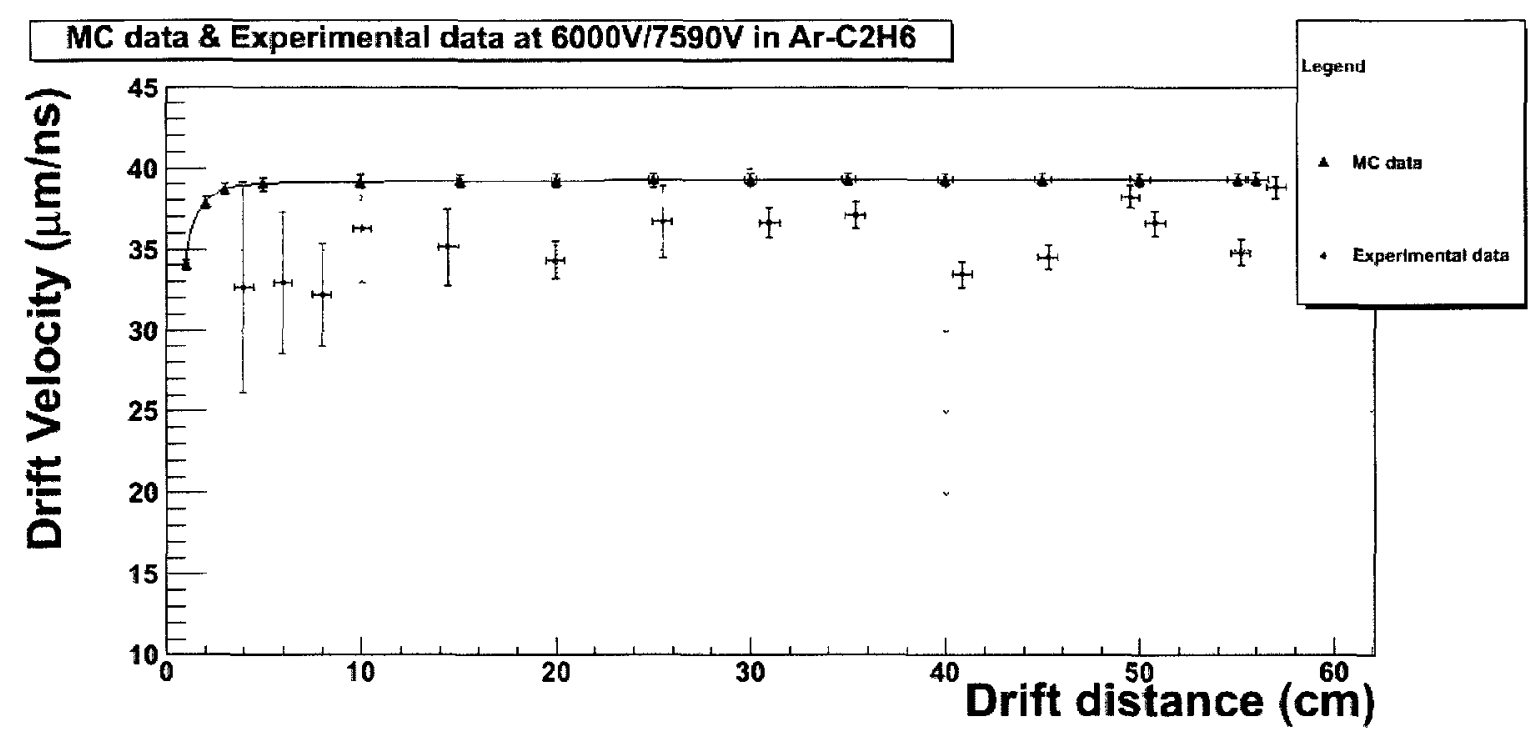

Figure 5.7: Drift velocity curve for $6000 \mathrm{~V}$

\section{3 $\quad \mathrm{Ar}-\mathrm{C}_{2} \mathrm{H}_{2}-\mathrm{C}_{2} \mathrm{H}_{6}$}

\subsection{1 $4000 \mathrm{~V}$}

Ar- $\mathrm{C}_{2} \mathrm{H}_{2}-\mathrm{C}_{2} \mathrm{H}_{6}$, or 'trimix' as it is commonly referred to, is thought to be the ideal drift gas for the CRIPT prototypes. This is the case because it is a moderate speed gas with the lowest amounts of diffusion. Over the drift length of the detector at 4000 $\mathrm{V}$ and $6000 \mathrm{~V}$ the longitudinal diffusion will be $0.242 \mathrm{~cm}$ and $0.226 \mathrm{~cm}$ respectively. This is the diffusion in the direction of drift and causes the ionization to potentially arrive either earlier or later, depending on which direction (forwards or backwards) the majority of the diffusion occurs, than it would if diffusion simply did not occur. This is a source of error which will be discussed shortly. It is a good choice because of its stable behaviour over the length of the drift chamber.

Fig 5.8 shows the drift velocity over the drift distance. The mean of the experimental data is $10.18 \mu \mathrm{m} / \mathrm{ns}$ with a standard deviation of $0.62 \mu \mathrm{m} / \mathrm{ns}$. Despite the obvious disagreement between the experiment and the simulation the data are not spread over a large range. These data were taken between April 2011 and May 2011 


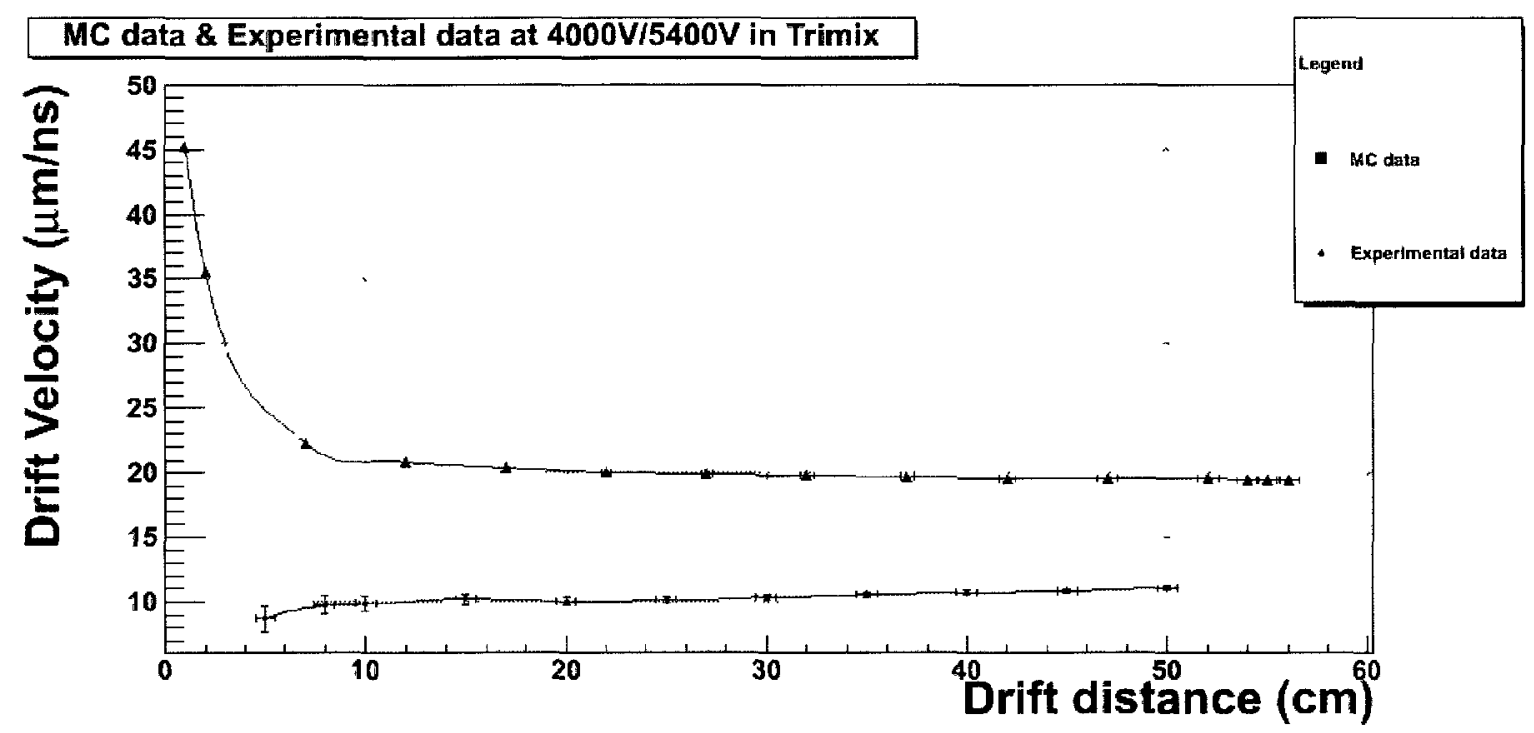

Figure 5.8: Drift velocity curve for $4000 \mathrm{~V}$. These data were likely taken after contamination occurred.

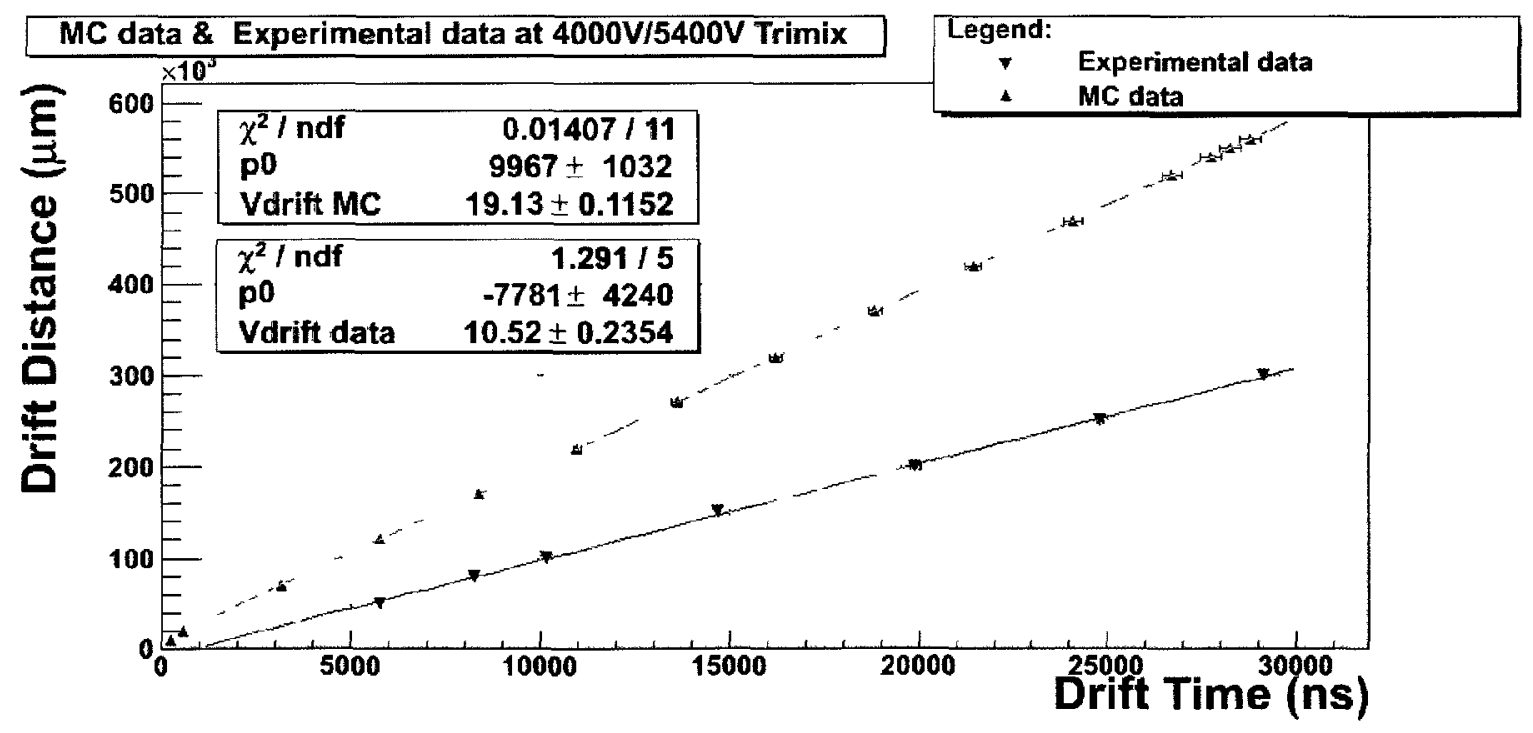

Figure 5.9: Linear plot for $4000 \mathrm{~V}$. These data were likely taken after contamination occurred. 


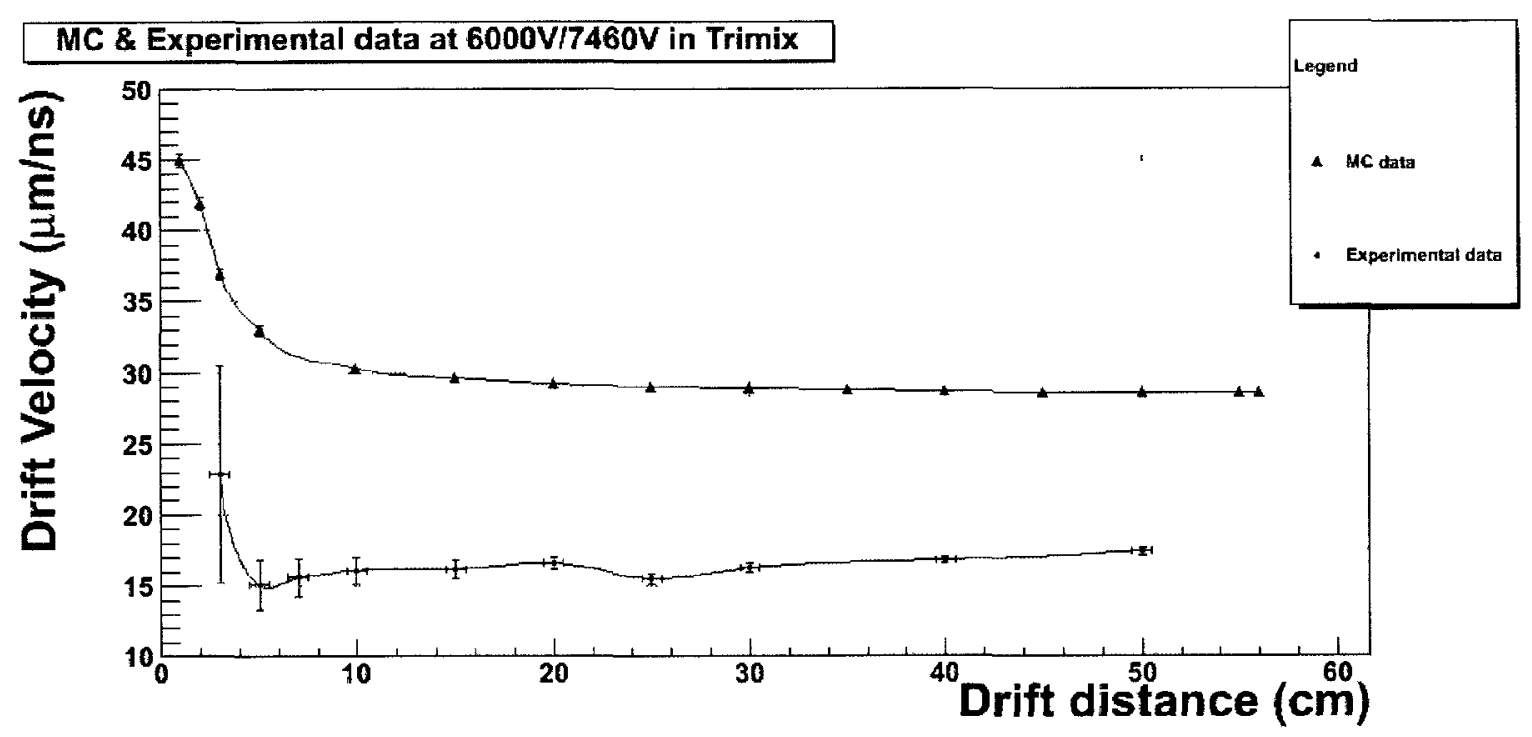

Figure 5.10: Drift velocity curve for $6000 \mathrm{~V}$. These data were likely taken after contamination occurred.

and the significance of the date will be discussed in a subsequent section. The linear plot of these same data is shown in fig 5.9. The results from this section will be summarized along with the results for the $6000 \mathrm{~V}$ data in the next subsection.

\subsection{2 $6000 \mathrm{~V}$}

The drift velocity curve for this gas at a cathode voltage of $6000 \mathrm{~V}$ is shown in fig 5.10. The same strange disagreement is seen here as with the lower voltage trimix data and the data for P10 at $3000 \mathrm{~V}$. This is again shown linearly in fig 5.11. These data were taken between May 2011 and June 2011.

The results for the trimix data are summarized in tables 5.3 and 5.4. 


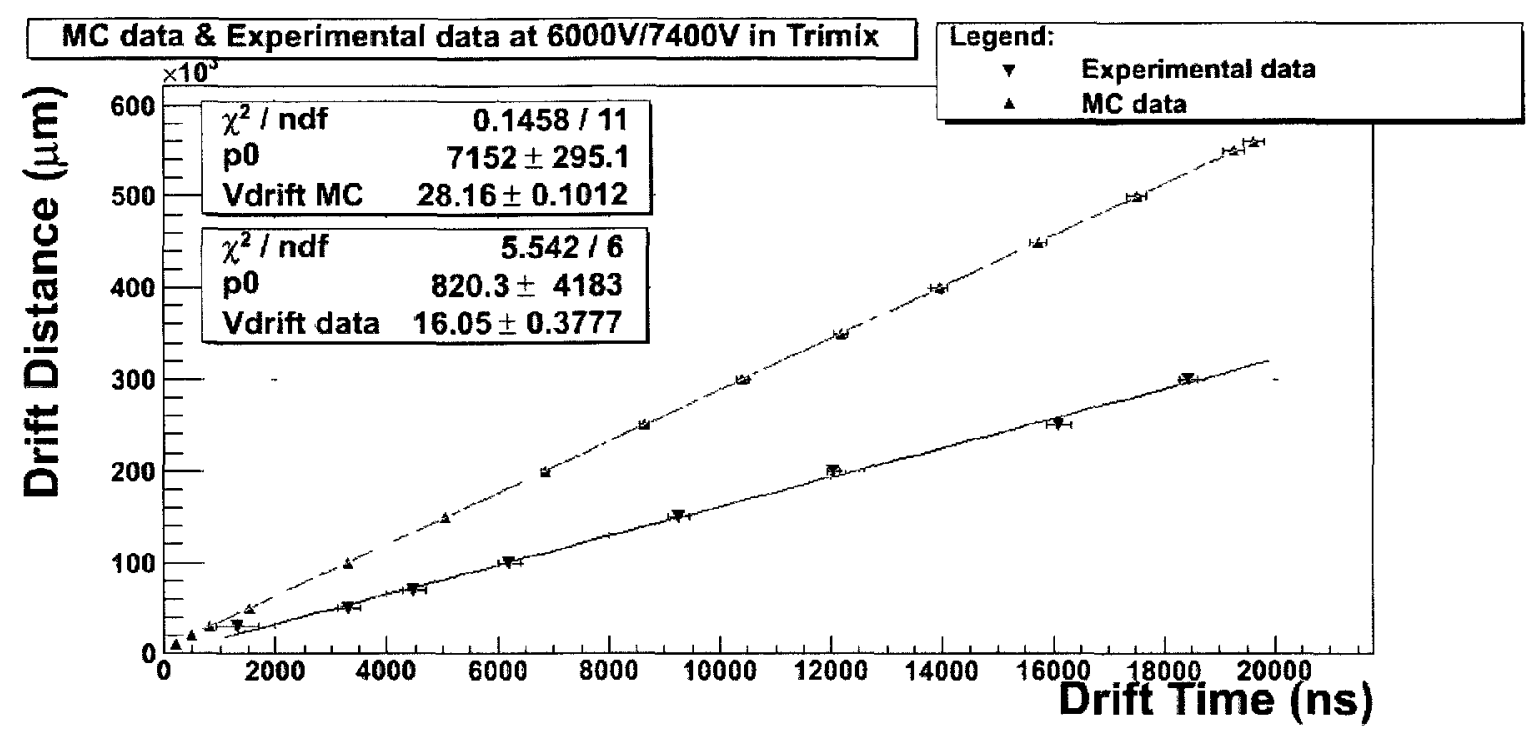

Figure 5.11: Linear plot for $6000 \mathrm{~V}$. These data were likely taken after contamination occurred.

\begin{tabular}{cccc}
\hline \hline Cathode Voltage & MC velocity & Experimental velocity & $\mathrm{t}_{\text {exp }}$ \\
\hline $4000 \mathrm{~V}$ & $19.54 \pm 0.08$ & $10.52 \pm 0.24$ & 0.6 \\
$6000 \mathrm{~V}$ & $28.16 \pm 0.10$ & $16.05 \pm 0.38$ & 0.55 \\
\hline \hline
\end{tabular}

Table 5.3: Summary of experimental results for trimix at $4 \mathrm{kV}$ and $6 \mathrm{kV}$ on the cathode. The units of drift velocity are $\mu \mathrm{m} / \mathrm{ns}$.

\begin{tabular}{ccc}
\hline \hline Cathode Voltage & MC $\chi_{\text {red }}^{2}$ & Experimental $\chi_{\text {red }}^{2}$ \\
\hline $4000 \mathrm{~V}$ & 0.00127 & 0.258 \\
$6000 \mathrm{~V}$ & 0.0132 & 0.924 \\
\hline \hline
\end{tabular}

Table 5.4: Summary of $\chi_{\text {red }}^{2}$ for trimix gas. 


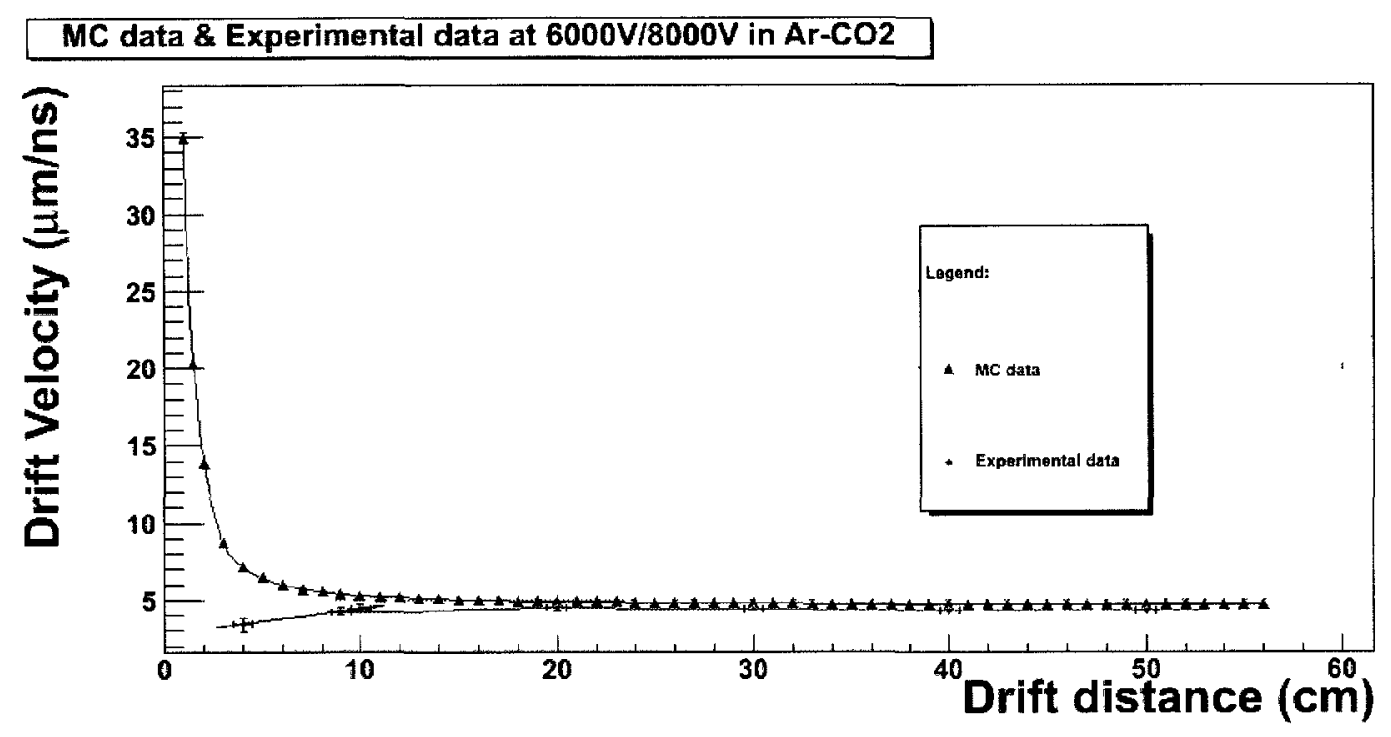

Figure 5.12: Drift velocity curve for $6000 \mathrm{~V}$

\subsection{Ar-CO2}

\subsection{1 $6000 \mathrm{~V}$}

As previously mentioned, this gas is quite slow and it was therefore not possible in the CRIPT prototypes to obtain data at a cathode voltage of $4 \mathrm{kV}$. Fig 5.12 shows the drift velocity curve for this gas. From the drift velocity curve the mean velocity is $4.22 \mu \mathrm{m} / \mathrm{ns}$ with a standard deviation of $0.34 \mu \mathrm{m} / \mathrm{ns}$. This agrees very well with the simulation results. It should be noted that there data were taken between February 2011 and March 2011.

\subsection{Summary of results}

For the sake of clarity the results of all data taken in my work will be summarized here. The most meaningful values to compare are the drift velocities acquired from the linear fits since they produce an average drift velocity with a small uncertainty due to the nature of the least squares fitting. Table 5.5 contains all necessary information. 


\begin{tabular}{lcccc}
\hline \hline & $\mathrm{Ar}-\mathrm{CH}_{4}$ & $\mathrm{Ar}-\mathrm{C}_{2} \mathrm{H}_{6}$ & $\mathrm{Ar}_{-} \mathrm{C}_{2} \mathrm{H}_{2}-\mathrm{C}_{2} \mathrm{H}_{6}$ & $\mathrm{Ar}-\mathrm{CO}_{2}$ \\
\hline $\mathrm{V}_{M C-3 k V}$ & $38.12 \pm 0.17$ & - & - & - \\
$\mathrm{V}_{E x p-3 k V}$ & $19.27 \pm 0.20$ & - & - & - \\
\hline $\mathrm{V}_{M C-4 k V}$ & $46.45 \pm 0.07$ & $33.34 \pm 0.10$ & $19.54 \pm 0.08$ & - \\
$\mathrm{V}_{E x p-4 k V}$ & $45.83 \pm 0.49$ & $31.26 \pm 0.23$ & $10.52 \pm 0.24$ & - \\
\hline $\mathrm{V}_{M C-6 k V}$ & $53.66 \pm 0.08$ & $39.63 \pm 0.17$ & $28.16 \pm 0.10$ & $4.64 \pm 0.01$ \\
$\mathrm{~V}_{E x p-6 k V}$ & $56.66 \pm 0.49$ & $37.55 \pm 0.33$ & $16.05 \pm 0.28$ & $4.38 \pm 0.05$ \\
\hline \hline
\end{tabular}

Table 5.5: Summary of experimental results. The units of drift velocity are $\mu \mathrm{m} / \mathrm{ns}$. The cathode voltages can be expressed in terms of their resulting drift fields in the following way: $3000 \mathrm{~V}$ corresponds to $52.6 \mathrm{~V} / \mathrm{cm}, 4000 \mathrm{~V}$ corresponds to $70.2 \mathrm{~V} / \mathrm{cm}$, and $6000 \mathrm{~V}$ corresponds to $105.2 \mathrm{~V} / \mathrm{cm}$.

As shown, all results are in reasonably good agreement with the simulation with the exception of P10 at $3 \mathrm{kV}$ and the trimix at both $4 \mathrm{kV}$ and $6 \mathrm{kV}$.

\begin{tabular}{ccccc}
\hline \hline & $\mathrm{Ar}-\mathrm{CH}_{4}$ & $\mathrm{Ar}_{2} \mathrm{C}_{2} \mathrm{H}_{6}$ & $\mathrm{Ar}-\mathrm{C}_{2} \mathrm{H}_{2}-\mathrm{C}_{2} \mathrm{H}_{6}$ & $\mathrm{Ar}-\mathrm{CO}_{2}$ \\
\hline $\mathrm{V}_{\text {Exp-3kV}}$ & May/June 2011 & - & - & - \\
\hline $\mathrm{V}_{\text {Exp-4kV}}$ & Jan/Feb 2011 & Feb/March 2011 & Apr/May 2011 & - \\
\hline $\mathrm{V}_{\text {Exp-6kV}}$ & Jan/Feb 2011 & Feb/March 2011 & May/June 2011 & Feb/March 2011 \\
\hline \hline
\end{tabular}

Table 5.6: Summary of experimental results by approximate date of data collection.

Table 5.6 summarizes the approximate dates when the experimental data for this research were taken. There is an explicit correlation between the agreement and the type of weather experienced seasonally during those times. The data taken as the average daytime temperature increased disagree significantly more so than data taken during the cold months of January, February, and March. 


\subsection{Effects of $\mathrm{H}_{2} \mathrm{O}$ contamination}

A possible explanation as to why some of the results do not agree with the simulation could be contamination of the drift gas with $\mathrm{H}_{2} \mathrm{O}$. The reason the approximate dates that data were taken over were given is because they span two vastly different seasons. This is significant because there is no humidity control in the laboratory where this work was carried out. Moreover, upon decommissioning the prototypes, it was discovered that water had leaked from the ceiling onto the detectors and scintillators in the apparatus.

This luckily did not destroy the detectors as they are supposed to be isolated from the atmosphere but unfortunately nothing is ever quite perfect and there is a chance, albeit small, that either humidity from the atmosphere or this extraneous water made its way into the drift volume thereby changing the exact chemical composition of the drift gas. This is relatively straightforward to examine. MAGBOLTZ is already perfectly suited for such a task and the SOR simulation of the detector can then interpret the MAGBOLTZ results and produce corrected drift velocities based on the appropriate concentration of contaminant. This will be examined for $\mathrm{Ar}-\mathrm{CH}_{4}$ and $\mathrm{Ar}-\mathrm{C}_{2} \mathrm{H}_{2}-\mathrm{C}_{2} \mathrm{H}_{6}$ as the results for the other gases do not show the obvious effects of contamination. Once an approximate average concentration of $\mathrm{H}_{2} \mathrm{O}$ has been established, plots including this concentration of $\mathrm{H}_{2} \mathrm{O}$ will be shown for the other gases in appendix A.

According to [22] for low electric fields ${ }^{2}$ even $0.1 \%$ contamination by water vapour can reduce the drift velocity by a factor of two. This is due to the electric dipole moment of the water molecule which changes the inelastic scattering cross section of electrons by increasing it. The contamination phenomenon was examined with MAGBOLTZ. The simulation produced the curves shown in fig 5.13 and fig 5.14 for

\footnotetext{
${ }^{2}$ Slightly lower than the range used by the CRIPT prototypes
} 
P10 and the trimix respectively. For the case of P10 at a cathode voltage of $3000 \mathrm{~V}$, the drift field would be $52.6 \mathrm{~V} / \mathrm{cm}$. As seen in fig 5.13 at a drift field of $52.6 \mathrm{~V} / \mathrm{cm}$ the drift velocity is decreased to a value approximately equal to what was measured in the laboratory when the concentration of water vapour is roughly $0.1 \%$. It is for this reason that I will assume that this is in fact the correct fraction of contamination and it will be investigated further. Similarly, for the trimix from fig 5.14 is can be seen that for a the $4000 \mathrm{~V}$ data the appropriate level of contamination is $0.3 \%$ and for $6000 \mathrm{~V}$ the level increases to $0.5 \%$. These are the MAGBOLTZ results that will be given to the SOR field simulation to produce the Monte Carlo drift velocities. 

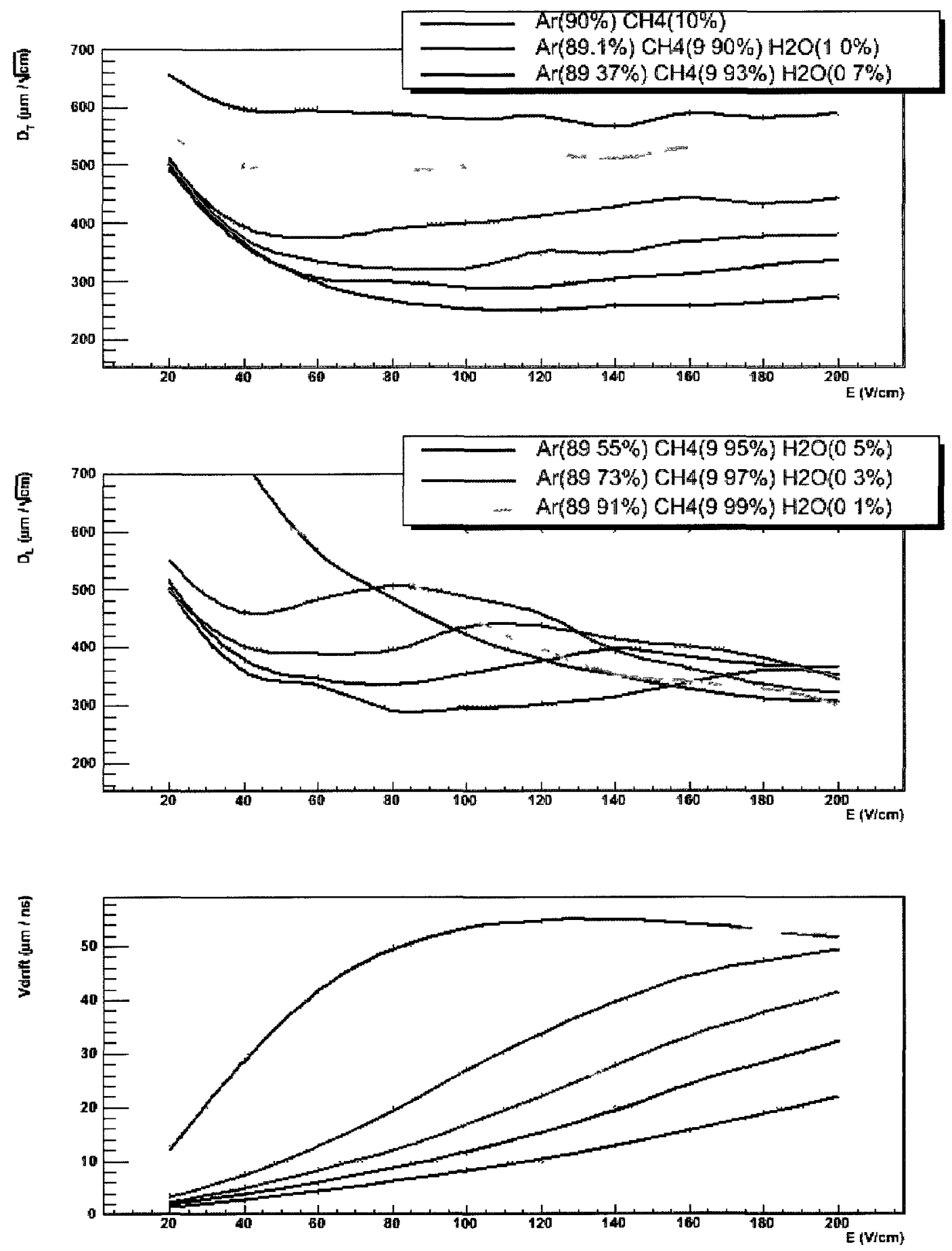

Figure 5.13: MAGBOLTZ results for $\mathrm{H}_{2} \mathrm{O}$ contamination in $\mathrm{ArCH}_{4}$ Top Transverse diffusion Middle Longitudinal diffusion Bottom Drift velocity 

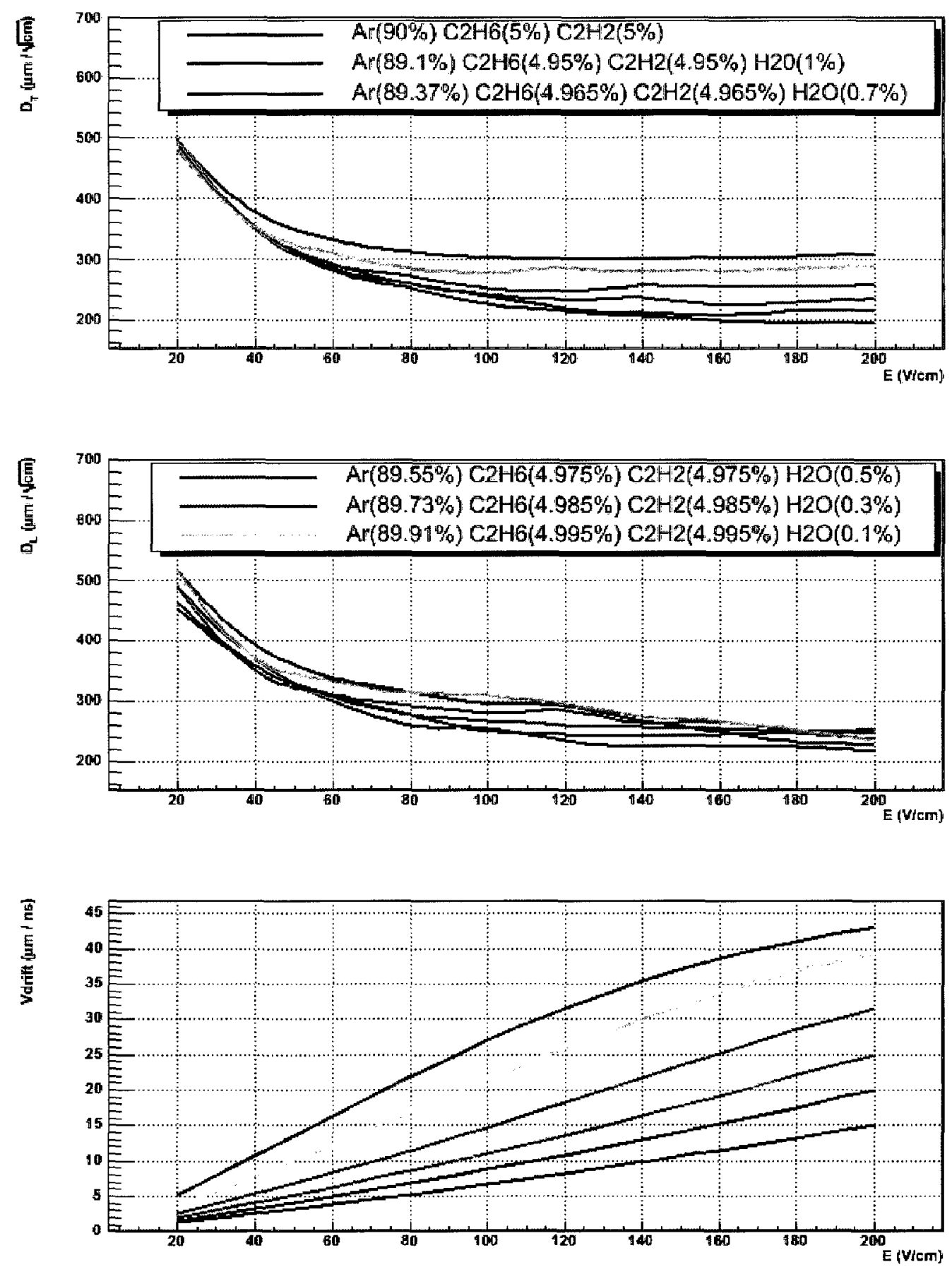

Figure 5.14: MAGBOLTZ results for $\mathrm{H}_{2} \mathrm{O}$ contamination in $\mathrm{ArC}_{2} \mathrm{H}_{2} \mathrm{C}_{2} \mathrm{H}_{6}$. Top: Transverse diffusion. Middle: Longitudinal diffusion. Bottom: Drift velocity. 


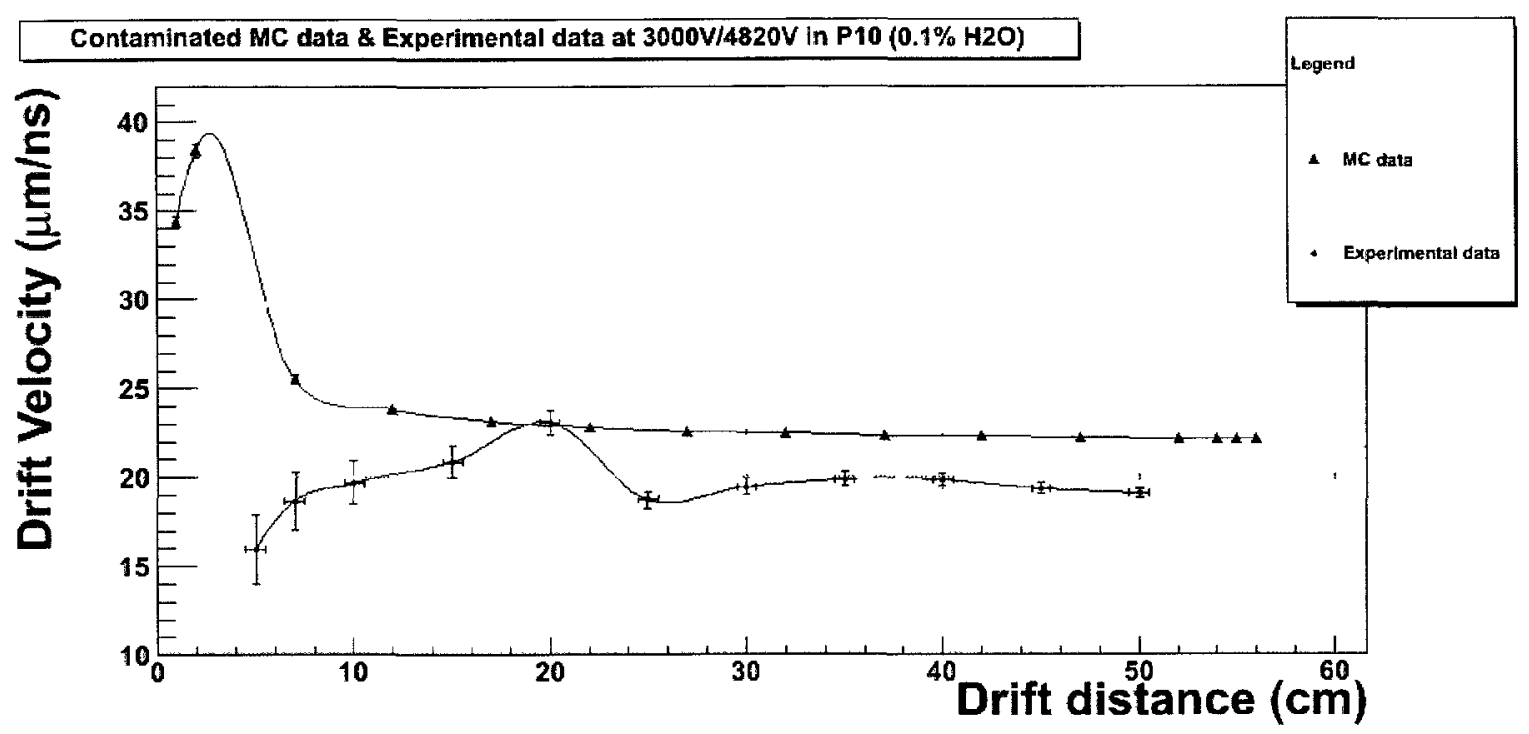

Figure 5.15: Drift velocity curve for argon-methane at $3000 \mathrm{~V}$ on the cathode with $0.1 \% \mathrm{H}_{2} \mathrm{O}$ vapour present.

\subsection{1 $\mathrm{Ar}-\mathrm{CH}_{4}$}

\section{$3000 \mathrm{~V}$}

The results previously presented for this gas at this particular drift field showed signs for concern. The large discrepancy between the measurements and what the SOR simulation of the detectors produced can be explained if real-world consideration of the environment is taken. It was shown in fig 5.13 that if $0.1 \%$ of water vapour is introduced to the drift gas the results, at least from MAGBOLTZ, are in better agreement. Given the drastic seasonal change and the evidence of water leaking onto the apparatus this is the beginning of an explanation. Fig 5.15 shows the drift velocity curve, with the same experimental data points as previous shown but accompanied by SOR Monte Carlo data for the drift velocity as well. The simulated data now lie within $2 \sigma$ of the mean of the experimental results.

The linear plots for these data series are shown fig 5.16. The results are summarized in table 5.7. The previous ' $\mathrm{t}$ ' value between experiment and theory was 0.65 while it is reduced to 0.12 when the contamination is introduced. The improvement 


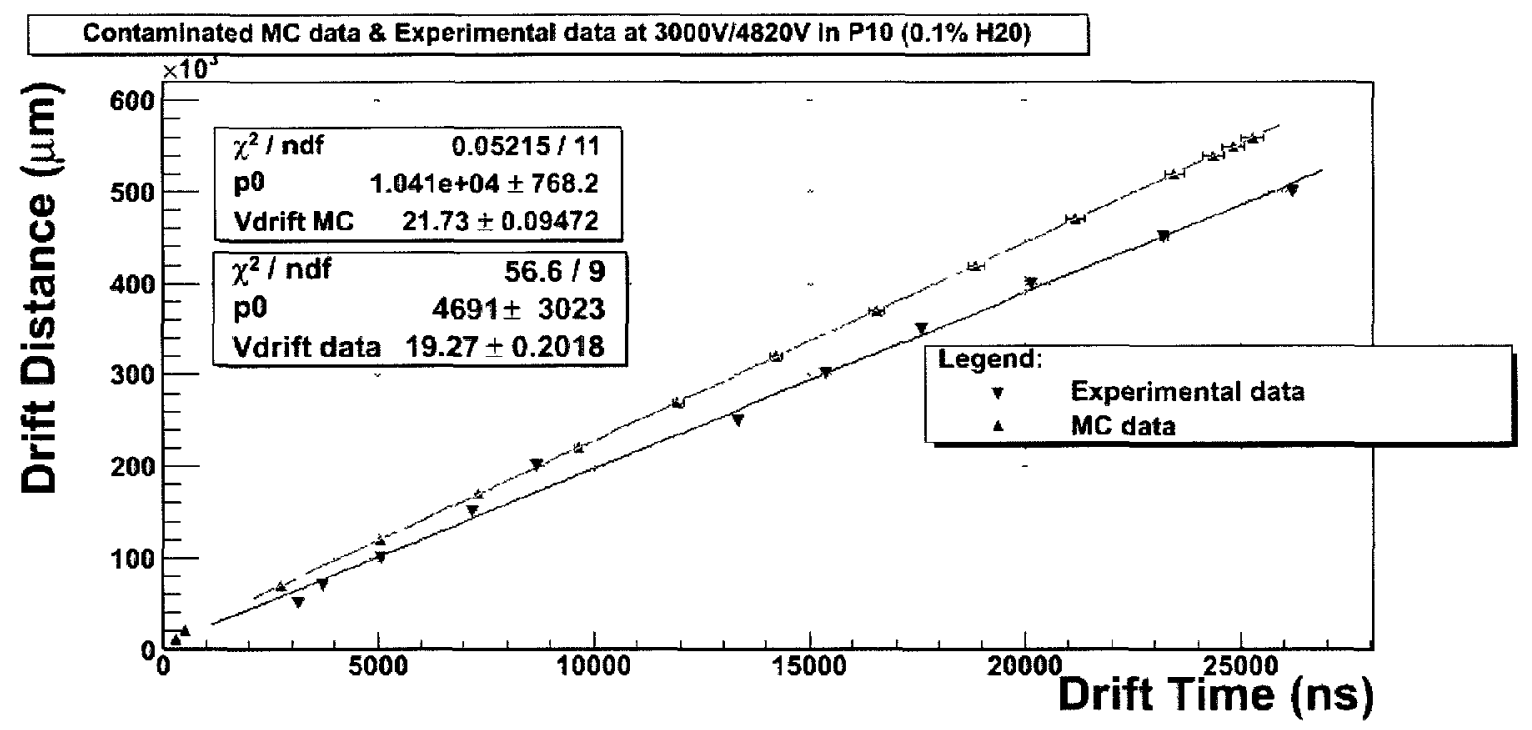

Figure 5.16: Linear plot for argon-methane at $3000 \mathrm{~V}$ on the cathode with $0.1 \%$ $\mathrm{H}_{2} \mathrm{O}$ vapour present.

manifests as a 5 fold reduction in the average difference.

\begin{tabular}{cccc}
\hline \hline Cathode Voltage & MC velocity & Experimental velocity & $t_{\exp }$ \\
\hline $3000 \mathrm{~V}$ & $21.73 \pm 0.09$ & $19.27 \pm 0.20$ & 0.12 \\
\hline \hline
\end{tabular}

Table 5.7: Summary of experimental results for $\mathrm{P} 10$ with $3 \mathrm{kV}$ on the cathode with $0.1 \%$ contamination with $\mathrm{H}_{2} \mathrm{O}$. The units of drift velocity are $\mu \mathrm{m} / \mathrm{ns}$. 


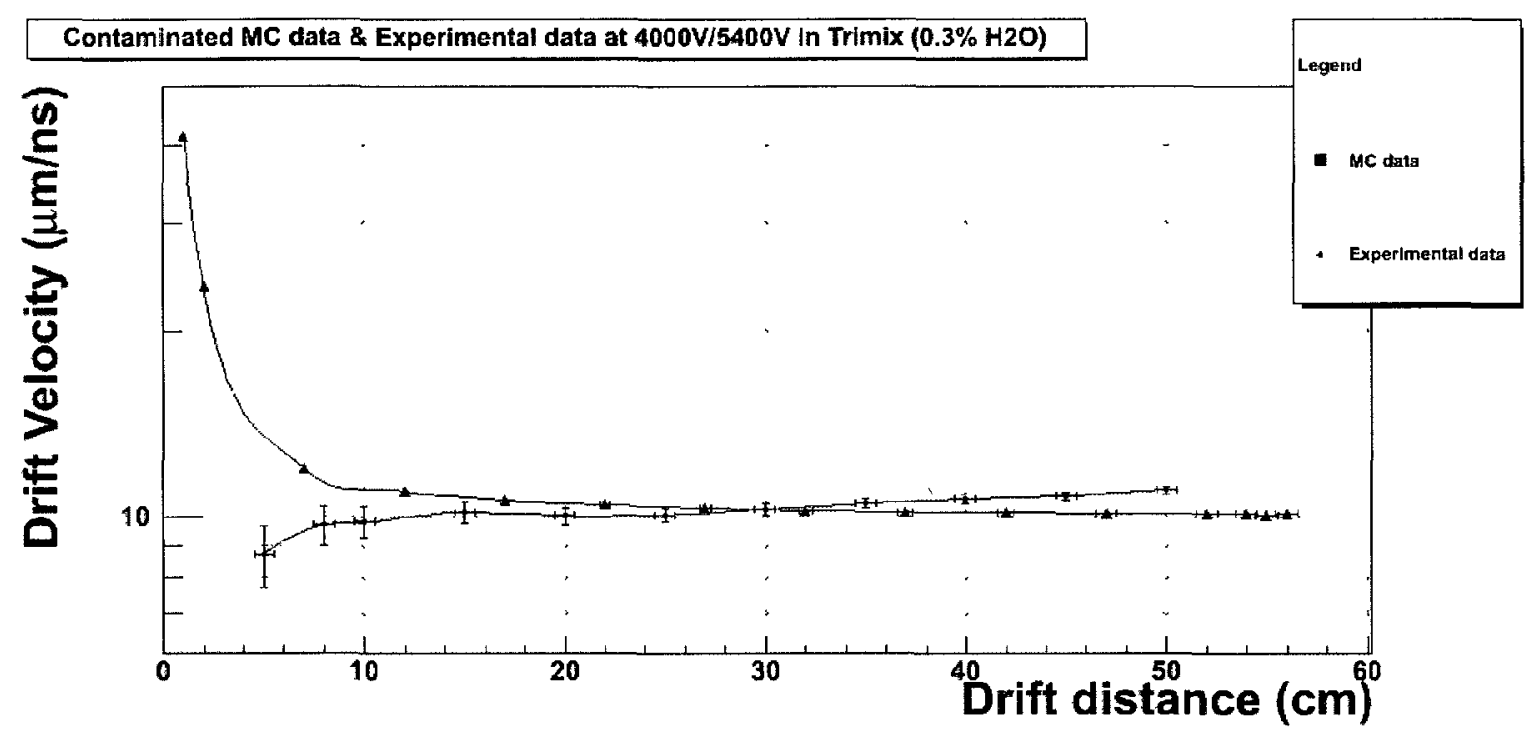

Figure 5.17: Drift velocity curve for trimix at $4000 \mathrm{~V}$ on the cathode with $0.3 \%$ $\mathrm{H}_{2} \mathrm{O}$ vapour present. The vertical scale is plotted on a $\log$ axis.

\subsection{2 $\quad \mathrm{Ar}-\mathrm{C}_{2} \mathrm{H}_{2}-\mathrm{C}_{2} \mathrm{H}_{6}$}

\section{$4000 \mathrm{~V}$}

The same reasoning is then applied to the trimix at $4000 \mathrm{~V}$ except as seen in fig 5.14 best agreement seems to be roughly $0.3 \%$ contamination with $\mathrm{H}_{2} \mathrm{O}$ vapour. Passing the MAGBOLTZ results to the SOR simulation produces the drift velocity curve shown in fig 5.17 and the linear plot shown in fig 5.18. The agreement from the drift velocity curve is within ${ }^{3} 1 \sigma$ which signifies excellent agreement. The results are summarized in table 5.8 .

\begin{tabular}{cccc}
\hline \hline Cathode Voltage & MC velocity & Experimental velocity & $\mathrm{t}_{\exp }$ \\
\hline $4000 \mathrm{~V}$ & $9.87 \pm 0.04$ & $11.19 \pm 0.12$ & 0.12 \\
\hline \hline
\end{tabular}

Table 5.8: Summary of experimental results for trimix at $4000 \mathrm{~V}$ on the cathode with $0.3 \%$ contamination with $\mathrm{H}_{2} \mathrm{O}$. The units of drift velocity are $\mu \mathrm{m} / \mathrm{ns}$.

\footnotetext{
${ }^{3}$ Agreement is seen from $10 \mathrm{~cm}$ of drift distance and up.
} 


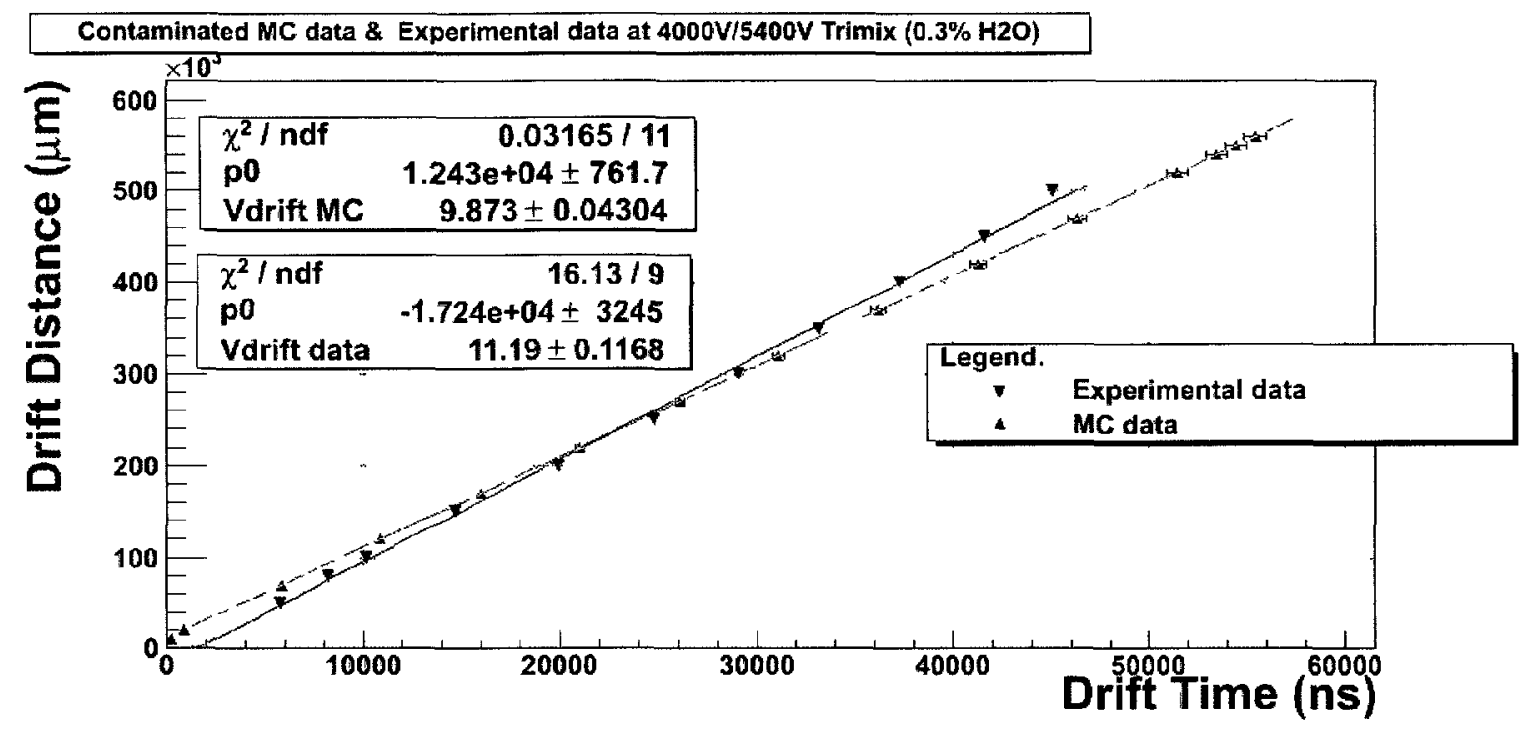

Figure 5.18: Linear plot for trimix at $4000 \mathrm{~V}$ on the cathode with $0.3 \% \mathrm{H}_{2} \mathrm{O}$ vapour present.

As the simulated drift velocity is on average lower than the experimental result is can safely be assumed that the estimation of $0.3 \%$ contamination is too generous. The actual value is close to this but slightly lower.

\section{$6000 \mathrm{~V}$}

Lastly, this very same logic is applied to the final drift field that showed disagreement originally. Again, the MAGBOTLZ simulation seems to suggest that in this instance the fraction of contamination is approaching $0.5 \%$. One possible explanation is that the days over which this particular data series was obtained may have been unusually humid which would exacerbate the already present signs of water near the experiment.

The results of this examination are shown as the drift velocity curve in fig 5.19 and the linear plot in fig 5.20 and subsequently summarized in table 5.9. Again, the experimental results are within $1 \sigma$ of the simulation for large drift distances. The contamination fraction may again have been generous but is very likely still higher than the $0.3 \%$ estimated for the $4000 \mathrm{~V}$ data. 


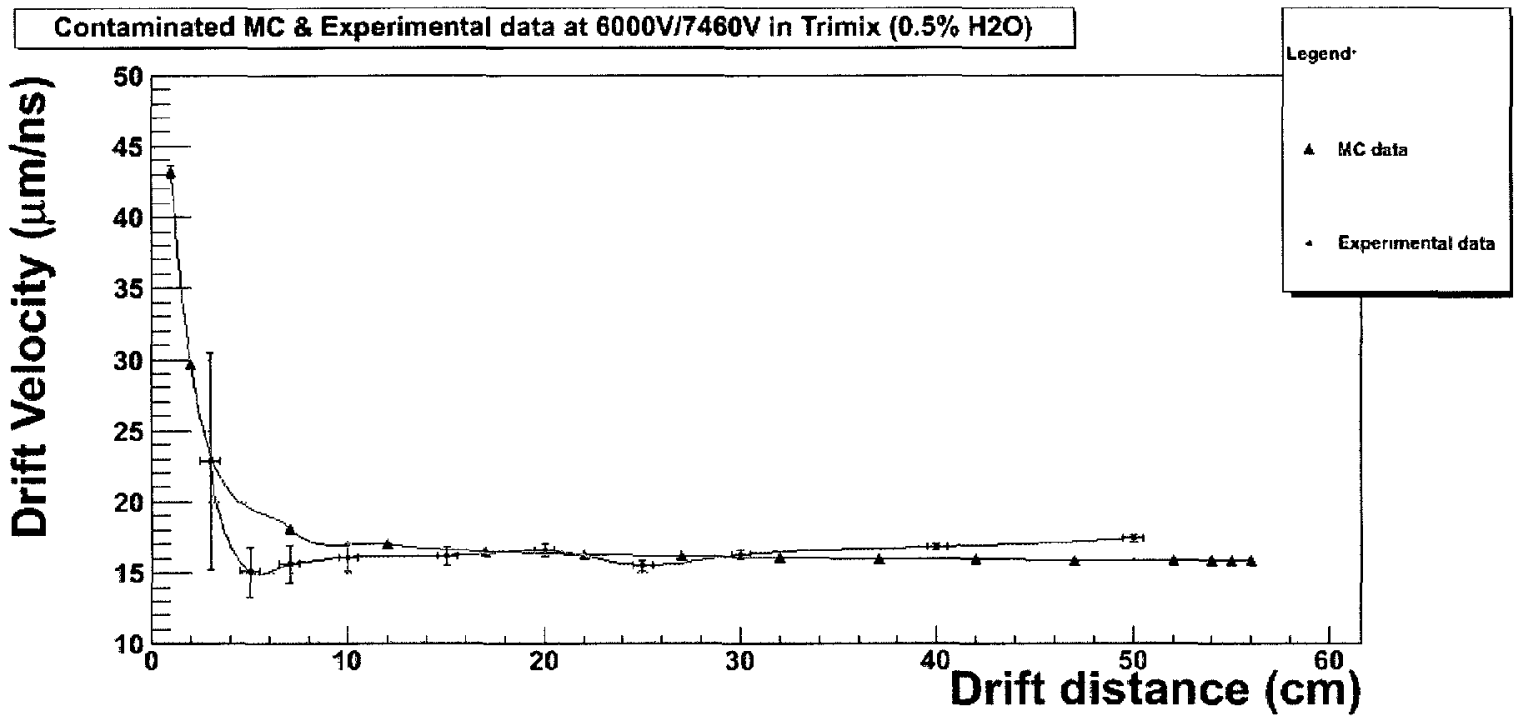

Figure 5.19: Drift velocity curve for trimix at $6000 \mathrm{~V}$ on the cathode with $0.5 \%$ $\mathrm{H}_{2} \mathrm{O}$ vapour present.

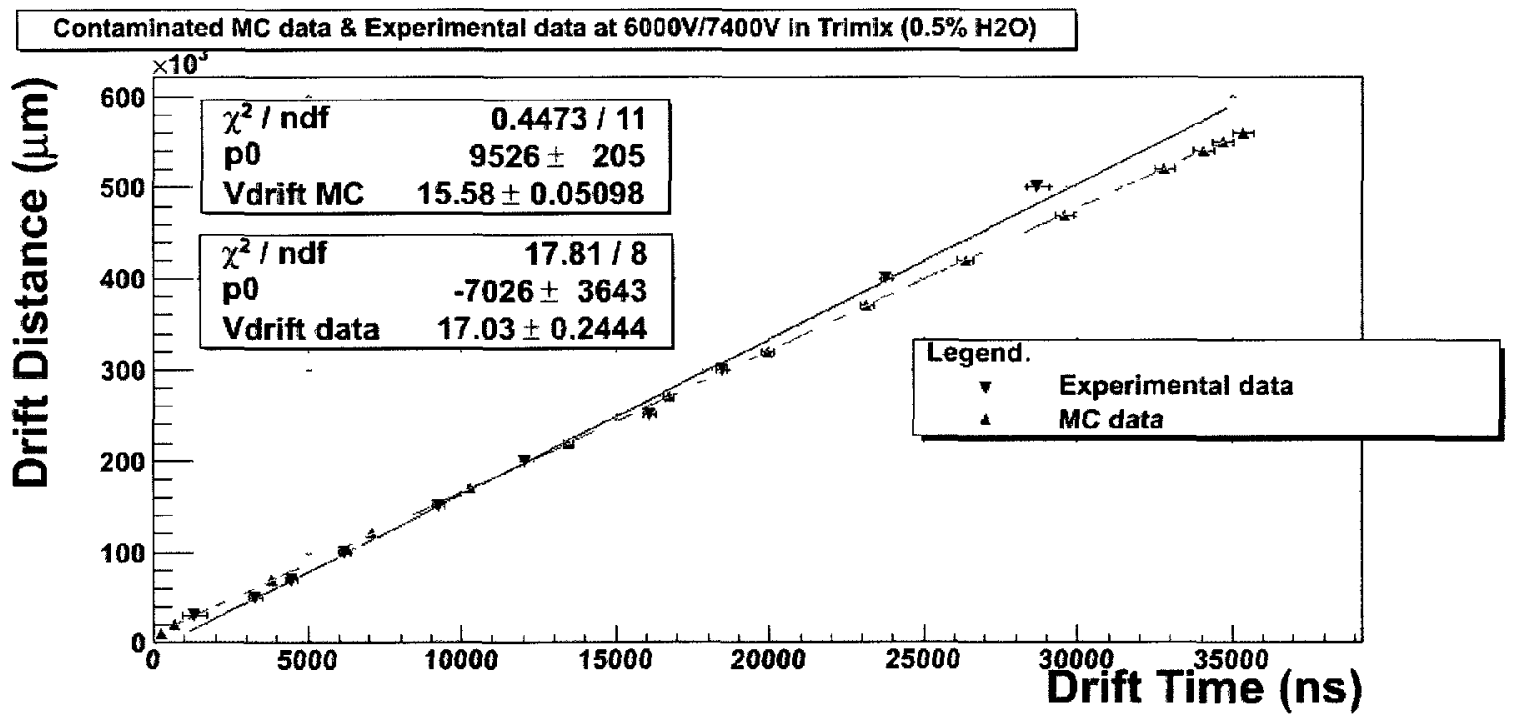

Figure 5.20: Linear plot for trimix at $6000 \mathrm{~V}$ on the cathode with $0.5 \% \mathrm{H}_{2} \mathrm{O}$ vapour present.

\begin{tabular}{cccc}
\hline \hline Cathode Voltage & MC velocity & Experimental velocity & $t_{\text {exp }}$ \\
\hline $6000 \mathrm{~V}$ & $15.58 \pm 0.05$ & $17.03 \pm 0.24$ & 0.0089 \\
\hline \hline
\end{tabular}

Table 5.9: Summary of experimental results for trimix at $6000 \mathrm{~V}$ on the cathode with $0.5 \%$ contamination with $\mathrm{H}_{2} \mathrm{O}$. The units of drift velocity are $\mu \mathrm{m} / \mathrm{ns}$. 


\subsubsection{Conclusion}

The fact that the drift chambers are easily, under the described circumstances, contaminated with $\mathrm{H}_{2} \mathrm{O}$ says nothing at all about their potential for use in future research endeavours. The drift velocity can be easily monitored in real-time as events are recorded by simply making the appropriate changes to the DAQ and analysis software. Therefore, as long as the drift velocity does not become so low that event pile up begins to occur, the actual magnitude is irrelevant. The real-time monitoring can warn future experimenters about issues before precious time and drift gas are wasted. Moreover, since position reconstruction depends explicitly on the drift velocity, it can be done much more accurately with the drift velocity curves depending on how this method is implemented. With adequate samples of the velocity over the drift distance simple spline interpolation can effectively fill in the gaps between data points.

\subsection{Experimental error analysis}

\begin{tabular}{ccc}
\hline \hline Time & Distance & Velocity \\
\hline DAQ bias & Scintillator position & MAGBOLTZ \\
Signal cables & Scintillator angle & SOR flight oscillations \\
Hardware & & \\
Pulse fitting & & \\
Timing histogram fitting & \\
Scintillator sample window & \\
\hline \hline
\end{tabular}

Table 5.10: Summary of the sources of error 
Experimental research is inevitably faced with the notions of error and uncertainty. The research presented in this thesis is absolutely subject to systematic and statistical error along with fundamental limitations of accuracy presented by various experimental elements. The sources of error present in this experiment are shown in table 5.10 .

The DAQ system is subject to the fundamental limitation of its sampling frequency which is $100 \mathrm{~ns}$. This means that for each signal received from the detector there is an inherent uncertainty of $100 \mathrm{~ns}$. This is the minimum resolvable time the software/hardware components can make. For the purposes of the experiment presented here this uncertainty is quite small and there are other, more significant sources of error to account for.

The signal cables used to connect components, specifically the detector and the DAQ, the PMTs and the NIM modules, or the connections between the NIM modules themselves all contribute a finite error to the measurement of the time. Typically cables contribute a lag or delay from one end to the other to the amount of $1 \mathrm{~ns}$ per foot of length. This was taken into consideration from the very beginning. The connections between the NIM modules all use very similar short lengths of cable and the signal cables from the preamplifier board on the detector are identical. The largest contribution from cable timing error is the PMT signal cables. Three of the four are approximately the same length ( 5 feet or less) whereas the $4^{\text {th }}$ scintillator - the one used with the topmost scintillator to attenuate the illumination of the drift region as described in the GD section was required to be longer. The theoretical flux for cosmic ray muons seen by the CRIPT cosmic ray telescope trigger should be roughly $0.78 \mu / \mathrm{s}$. It was consistently observed that the detected flux with the GD method was on average in this same range. If the geometry is taken into account the flux should be significantly lower. This is a sign that the PMT trigger pulses are triggering on spurious cosmic ray events. This is seen in the timing histogram as the noise in 
the regions outside of the Gaussian fitting. This is easily overcome by the statistics required to accurately fit the Gaussian in the first place.

The NIM modules themselves cannot possibly input and output information instantaneously. The design parameters of these instruments state that they operate at sampling frequencies greater than $100 \mathrm{MHz}$ [20]. This error is so small it does not contribute appreciably to the experimental results.

The fitting of the raw data pulses provides a source of error. The uncertainty of the DAQ is the root of this error and is quantized as follows. The minimum bin size for the pulse histograms is $100 \mathrm{~ns}$ as dictated by the DAQ. It is therefore appropriate to associate an error of $\frac{100 n s}{\sqrt{12}}$ to the value chosen as 't0' from this pulse. When this quantity is added in quadrature with the statistical fitting errors it becomes negligible.

The Gaussian fit to the GD attenuated timing histogram is therefore subject primarily to statistical error associated with the fitting routine applied with ROOT. The reduced chi-squared for these fits is typically between 1.1 and 3 depending on the drift gas and the distance the sample is from the anode wire. The distance broadens the Gaussian by diffusion that occurs over the length of drift. The longitudinal diffusion can either be parallel or anti-parallel to the drift direction vector meaning that the ionization can either arrive sooner or later than it would without diffusion occurring. Despite there being no experimental correlation to the amount of diffusion occurring the GD timing histograms consistently had a definite central peak which corresponded to what the fitting routine calculated.

The width of the GD scintillator overlap is a source of uncertainty because the drift velocity can change drastically over a range of $4 \mathrm{~cm}$. This is typically seen for small drift distances as the electric field contribution from the anode wire becomes increasingly significant. This is a reason why some slight disagreement is seen in this region between the simulated data and the experimental data. 
The position error associated with the overlapping scintillators is finite and uniformly distributed. A meter stick was used to measure the distance of the center of this $4 \mathrm{~cm}$ overlap to the anode wire's location as marked on the upper lid of the detector. The meter stick has a reading error of $0.5 \mathrm{~mm}$ but to account for possible error in either the location of the anode wire or the mean position of the overlap the uncertainty is taken as $0.5 \mathrm{~cm}$. This is a significant source of measurement error and is reflected in the equation used to propagate the error from reading to measured and calculated quantities.

The overlap of scintillators might also not be perfectly parallel to the anode wire. The nature of the method involves mechanically moving them along the drift length to acquire data. Despite being secured together it is always possible that they shifted in position relative to one another. If this overlap was not completely parallel to the wire it would have the effect of broadening the Gaussian fit to the timing histogram. Due to not having a satisfactory method of measuring the longitudinal diffusion it cannot be said how significantly this effect is seen as it also depends on how rapidly the drift velocity changes in the $4 \mathrm{~cm}$ overlap region.

The error associated with the simulations, MAGBOLTZ and the SOR method, are finite. MAGBOLTZ is a FORTRAN based Monte Carlo program that is able to calculate uncertainties for the quantities under investigation. The uncertainty for the drift velocity varies over the ranges of electric field but is consistently less than $1 \%$ error. As seen in the section about the SOR simulation very small oscillations occur along the flight path of the ionization as it moves through the drift volume. These oscillations are very small but still apparent in the detector. One assumption made with the generation of Monte Carlo drift velocities from this method is that the fastest ionization always traverses the anode wire plane in the detector. Here the oscillations are minimal but the issue is that this is not always the path of the fastest ionization. The fastest path depends on the angle of incidence that the cosmic ray 
muon impinges upon the detector with. This assumption requires slight compensation when quoting the Monte Carlo results. An uncertainty of $1 \%$ was associated with every Monte Carlo drift velocity value and uncertainty of $0.5 \mathrm{~cm}$ was associated with their positions.

For the data presented in this thesis the uncertainty of the values is calculated as follows. For the AAR method the error bars derive from eqn 5.1.

$$
\sigma_{V}=\sqrt{\sigma_{t_{m a x}}^{2}+\left(\frac{\ln (2)}{C_{2}^{2}}\right)^{2} \sigma_{C_{2}}^{2}}
$$

Here $\sigma_{t_{\max }}$ is half of the arbitrary choice of bin size that this method requires, $\mathrm{C}_{2}$ the exponential coefficient from the general equation for exponential decay given by $e^{C_{2} x}$ which is the function used to fit the shoulder seen in the AAR timing histograms. This term is analogous to the half life of a typical exponentially decaying system. The time taken from this fit is where the exponential has reached half of its initial value. If $y=\frac{1}{2}=e^{C_{2} x}$ then solving for the time one obtains $\ln (2) / C_{2}$ which has units of $1 / \mathrm{s}$. The variable ' $\mathrm{t}$ ' used here is not the same as the quantity used to compare the experimental and Monte Carlo drift velocities as previously seen.

The error bars for the GD data derive from eqn 5.2 .

$$
\sigma_{V}=\frac{1}{t^{2}} \sqrt{t^{2} \sigma_{x}^{2}+x^{2} \sigma_{t}^{2}}
$$

Here ' $t$ ' is the mean of the Gaussian fit, $\sigma_{x}$ is the uncertainty of the position measurement, and $\sigma_{t}$ is the error on the Gaussian fit to the histogram. This expression comes from the application of the chain rule to the definition of Newtonian velocity. This expression is $\sigma_{V}^{2}=\left(\frac{\delta V}{\delta x}\right)^{2} \sigma_{x^{2}}+\left(\frac{\delta V}{\delta t}\right)^{2} \sigma_{t^{2}}$ which when simplified for elegance becomes eqn 5.2 . 


\section{Chapter 6}

\section{Summary}

This thesis was undertaken as part of the CRIPT collaboration in order to perform research and development concerning the application of prototype drift chambers assembled at Carleton University to meet the requirements for use in a real-time muon tomography apparatus. One part of determining the suitability of the drift chambers was to determine their position resolution in the directions both parallel and perpendicular to the anode wire. The work shown in this thesis is intimately connected to the work done by the CRIPT group at Carleton in determining the perpendicular position resolution. The resolution in this direction cannot be determined without reasonably accurate data concerning the drift velocity over the length of drift in the detector.

In order to cover this subject adequately the physics of cosmic ray muons was explained insofar as it applied to the research presented. The necessary instrumentation was covered and the experimental techniques, both novel and common, were described in detail as well. Candidate drift gases and magnitudes of electric fields at which the CRIPT prototypes can operate were probed and the drift velocity was

measured both as a function of distance and also as an average over the entirety of the drift length. One method provides accurate information regarding the nature of the drift velocity curves whereas the other provides a statistically reliable average 
value which can be used for comparing the different gases and drift fields.

It was found that the drift volume had potentially become contaminated with various fractions of water vapour and this phenomenon was examined in detail with the very same simulation tools the "ideal" data were derived from. Very good agreement was found when $\mathrm{H}_{2} \mathrm{O}$ contamination in small fractions was introduced leading this author to believe that it did indeed occur to some extent. Despite this experimental observation the prototypes can still potentially be put to future use if the drift velocity is measured in real time. 


\section{References}

[1] CBSA, Sea port operations, 2011, [Used with permission from CBSA Science and Engineering Directorate].

[2] K. Nakamura et al., Journal of Physics G 37, 075021 (2010).

[3] Wikipedia, Muon, http://en.wikipedia.org/wiki/Muon, 2011, [Online; accessed 10-Jun-2011].

[4] T. Sanuki, Nuclear Physics B - Proceedings Supplements 175-176, 149 (2008), proceedings of the XIV International Symposium on Very High Energy Cosmic Ray Interactions.

[5] V. Strickland, Technical report, Carleton University, Dept. of Physics, Ottawa (unpublished).

[6] D. Bryman, J. Bueno, and Z. Liu, Technical report, AAPS, Vancouver (unpublished).

[7] J. Allison et al., Nuclear Instruments and Methods in Physics Research Section A: Accelerators, Spectrometers, Detectors and Associated Equipment 310, 527 (1991).

[8] DFAIT, The Nuclear Non-Proliferation Treaty, http://www.international.gc. $\mathrm{ca} /$ arms-armes/nuclear-nucleaire/npt-tnp.aspx?lang=eng, 2011, [Online; accessed 17-Jun-2011].

[9] C. L. Morris et al., Science and Global Securtity 16, 37 (2008).

[10] J. Pater and R. Barlow, A Drift Chamber System for muon tomography, http: //www.hep.man.ac.uk/u/roger/, 2010, [Online; accessed 14-Jul-2011].

[11] S. Biagi, Magboltz - transport of electrons in gas mixtures, http://consult. cern.ch/writeup/magboltz/, 1995, [Online; accessed 23-May-2011]. 
[12] J. R. Hrandel, Advances in Space Research 38, 1549 (2006).

[13] A. Erlykin and A. Wolfendale, Astroparticle Physics 25, 183 (2006).

[14] L. Anchordoqui et al., Annals of Physics 314, 145 (2004).

[15] A. Robichaud, Master's thesis, Carleton University, Dept. of Physics, 2010.

[16] Y.-S. Tsai, Rev. Mod. Phys. 46, 815 (1974).

[17] H. A. Bethe, Phys. Rev. 89, 1256 (1953).

[18] C. Zorn, Radiation Physics and Chemistry 41, 37 (1993).

[19] H. Spieler, III. Scintillation Detectors, www-physics.1bl.gov/ spieler/physics_ 198_notes/PDF/III-Scint.pdf, 1998, [Online; accessed 11-Jun-2011].

[20] LecROY, NIM Model 465 Triple 4-Fold Logic Unit, http://www-esd.fnal.gov/ esd/catalog/main/lcrynim/465-spec.htm, 1998, [Online; accessed 9-Jun-2011].

[21] R. Fernow, Introduction to experimental partıcle physıcs (Cambridge, New York, 1989).

[22] W. Blum, W. Riegler, and L. Rolandi, Partıcle Detectıon with Drift Chambers (Springer, Berlin, 2008).

[23] W. R. Leo, Technıques for Nuclear and Partıcle Physics Experıments (SpringerVerlag, New York, 1994).

[24] R. Ritchie, Master's thesis, Carleton University, Dept. of Physics, 1986.

[25] W. W. Tsang and G. Marsaglia, Journal of Statistical Software 5, (undated).

[26] N. Drakos, The Successive Overrelaxation Method, http://www.netlib.org/ linalg/html_templates/node15.html, 1994, [Online; accessed 25-Jul-2011].

[27] M. Darwin, Relative Humidity over Last Year for Ottawa, http://ottawa. weatherstats.ca/charts/relative_humidity-1year.html, 2011, [Online; accessed 31-Jul-2011].

[28] M. Darwin, Temperature over Last Year for Ottawa, view-source:http:// ottawa. weatherstats.ca/charts/temperature-1year.html, 2011, [Online; accessed 31-Jul-2011]. 


\section{Appendix A}

\section{Further investigation of $\mathrm{H}_{2} \mathrm{O}$ \\ contamination with MAGBOLTZ}

Presented here are the MAGBOLTZ results for the remaining two CRIPT drift gases for which reasonable agreement was found initially. This contamination is simulated because it could represent the kind of results that would be obtained if the experiment was to be repeated.

\section{A.1 $\quad \mathrm{Ar}-\mathrm{C}_{2} \mathrm{H}_{6}$}

Shown in fig A.1 are the diffusion and drift velocity curves over a range of electric field strengths. The feature to make the most note of is that for certain fractions of $\mathrm{H}_{2} \mathrm{O}$ the longitudinal diffusion increases somewhat with stronger fields.

\section{A.2 $\quad \mathrm{Ar}-\mathrm{CO}_{2}$}

The MAGBOLTZ simulation for this gas mixture, shown in fig A.2, produces some interesting results for the diffusion values. It seems as though the diffusion is consistently reduced over increasing field strengths and concentrations of water. The downfall to this is of course the excessively low drift velocity. Over the range of water 

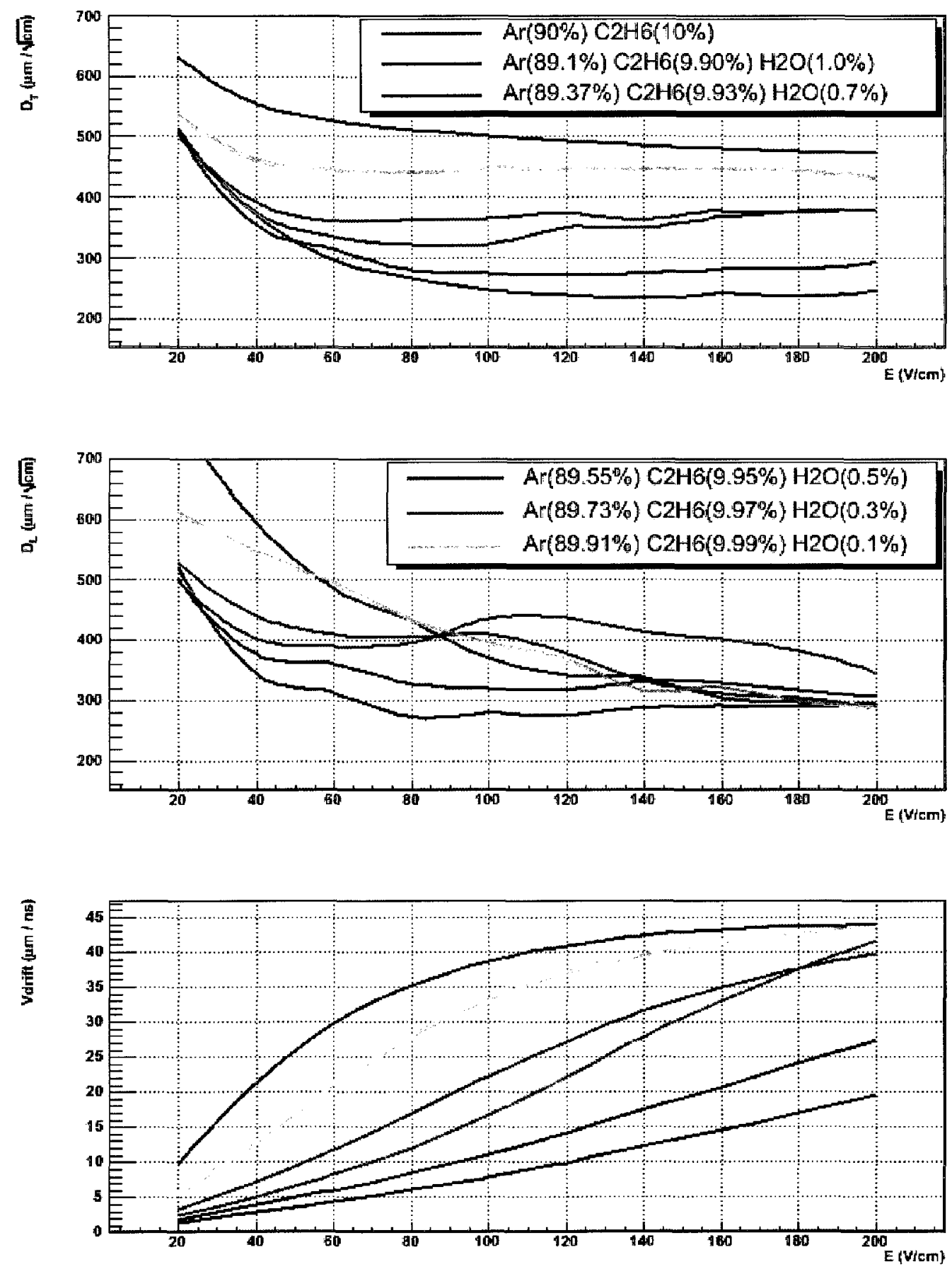

Figure A.1: MAGBOLTZ results for argon-ethane contaminated with water. Top: Transverse diffusion. Middle: Longitudinal diffusion. Bottom: Drift velocity 
concentrations shown the velocity reaches the practical limit for use in the CRIPT prototypes. At a field of $105 \mathrm{~V} / \mathrm{cm}$ with no contamination present the velocity is simulated to be approximately $4.5 \mu \mathrm{m} / \mathrm{ns}$ while at a concentration of $0.7 \%$ water the velocity drops to approximately $3 \mu \mathrm{m} / \mathrm{ns}$. As observed with what was assumed to be non-contaminated data at a drift field of $70 \mathrm{~V} / \mathrm{cm}$ the drift velocity is $3 \mu \mathrm{m} / \mathrm{ns}$ which was found to be too slow for the DAQ system to process the event pile-up. 

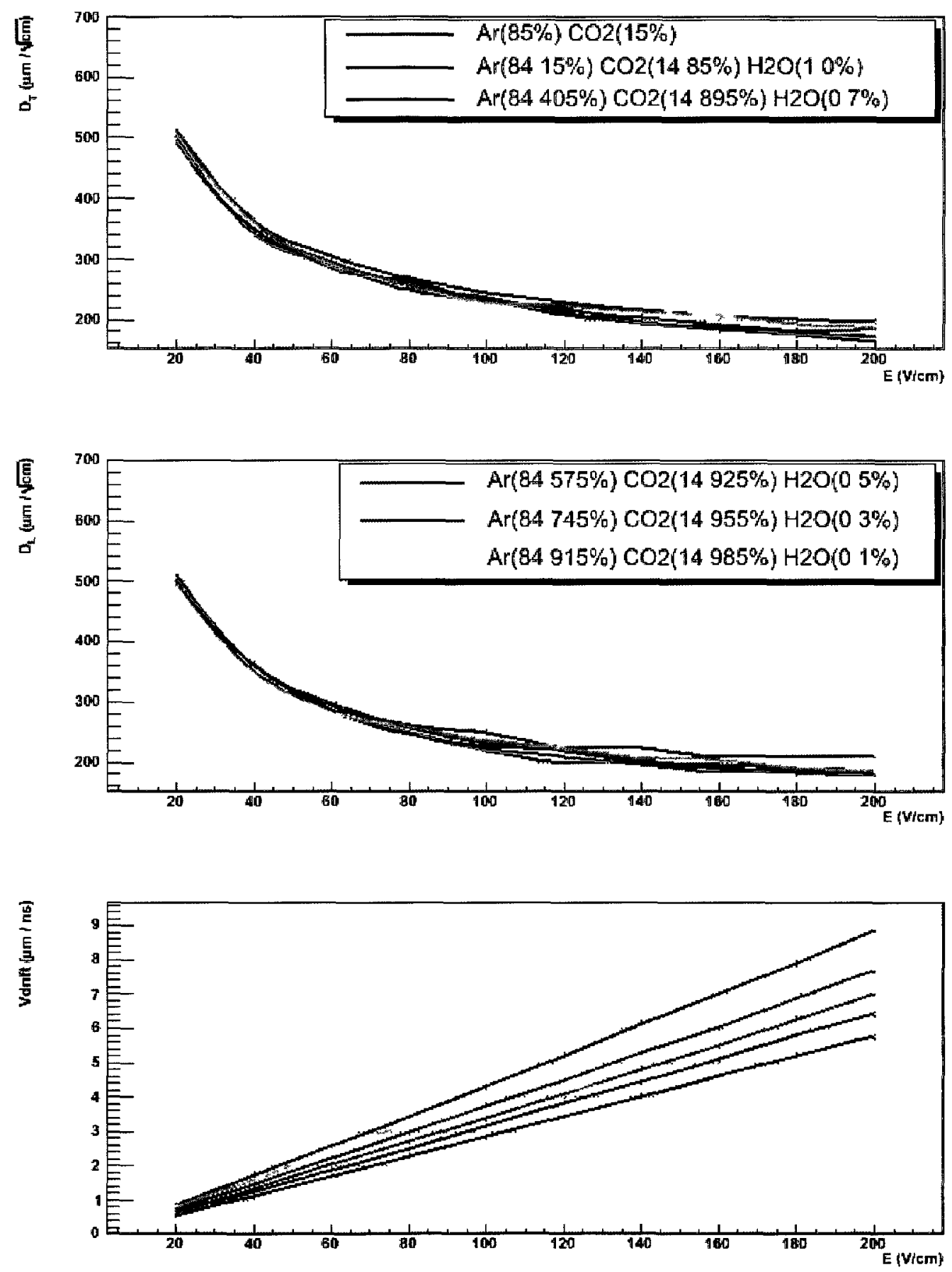

Figure A.2: MAGBOLTZ results for argon-carbon dioxide contaminated with water Top Transverse diffusion Middle Longitudinal diffusion Bottom Drift velocity 


\section{Appendix B}

\section{Investigation of air contamination with MAGBOLTZ}

\section{B.1 Ar-CH}

Presented here are the MAGBOLTZ results for the P10 gas with fractions of air as contaminants. The constituents of air are taken to be nitrogen (78.5\%) and oxygen (21.5\%) as they comprise the vast majority of compounds in air. The next significant compound is argon itself but since the gas mixture being simulated already consists of $90 \%$ (uncontaminated) argon this contribution is negligible. Moreover, the remaining elements in air exist in such low abundance that their contributions can safely be ignored.

Shown in fig B.1 are the diffusion and drift velocity curves over a range of electric field strengths. The fractions of air included as contaminant include, $0.1 \%, 0.3 \%$, $0.5 \%, 0.7 \%, 1 \%, 5 \%$, and $10 \%$. It can be seen that the drift velocity does indeed begin to decrease but this only happens at concentrations of air 50 times larger than the same concentrations of water. This means that for air to produce the same results as seen in the experimental data there would need to be a very significant fraction of the drift gas that is air. Leak tests were performed on the drift chamber and no 
leaks were found. Moreover, because the drift gas is constantly flowing through the detectors at approximately atmospheric pressure, the likelihood that air is able to consistently seep into the chamber is quite small. Therefore, water vapour at much lower concentrations, plays a more substantial role in attenuating the drift velocity to what was observed. This, coupled with the chronology of the experiment provide reason to believe that water vapour is more likely responsible for the observed drift velocities than air.

\section{B.2 $\mathrm{Ar}-\mathrm{C}_{2} \mathrm{H}_{6}$}

Fig B.2 shows the contamination curves produced by MAGBOLTZ for concentrations of $1 \%, 5 \%$, and $10 \%$ air. The drift velocity is reduced by a factor of approximately two when the concentrations of air are $5 \%$ or greater. Concentrations of this magnitude of contaminants would only be possible with a significant leak in the detector.

\section{B.3 $\mathrm{Ar}-\mathrm{C}_{2} \mathrm{H}_{6}-\mathrm{C}_{2} \mathrm{H}_{2}$}

Fig B.3 shows the MAGBOLTZ results for the trimix contaminated with the same fractions of air as seen for argon-ethane. The observed drift velocities in this gas mixture at $4 \mathrm{kV}$ and $6 \mathrm{kV}$ were $10.52 \pm 0.24$ and $16.05 \pm 0.28$ respectively. These correspond to drift fields on the MAGBOLTZ curves of $70.2 \mathrm{~V} / \mathrm{cm}$ and $105.2 \mathrm{~V} / \mathrm{cm}$. It can immediately be seen that for air to reduce the drift velocity to these experimental values the concentration of contamination would have to be greater than $10 \%$. This is far too substantial to result from a simple slow leak. 

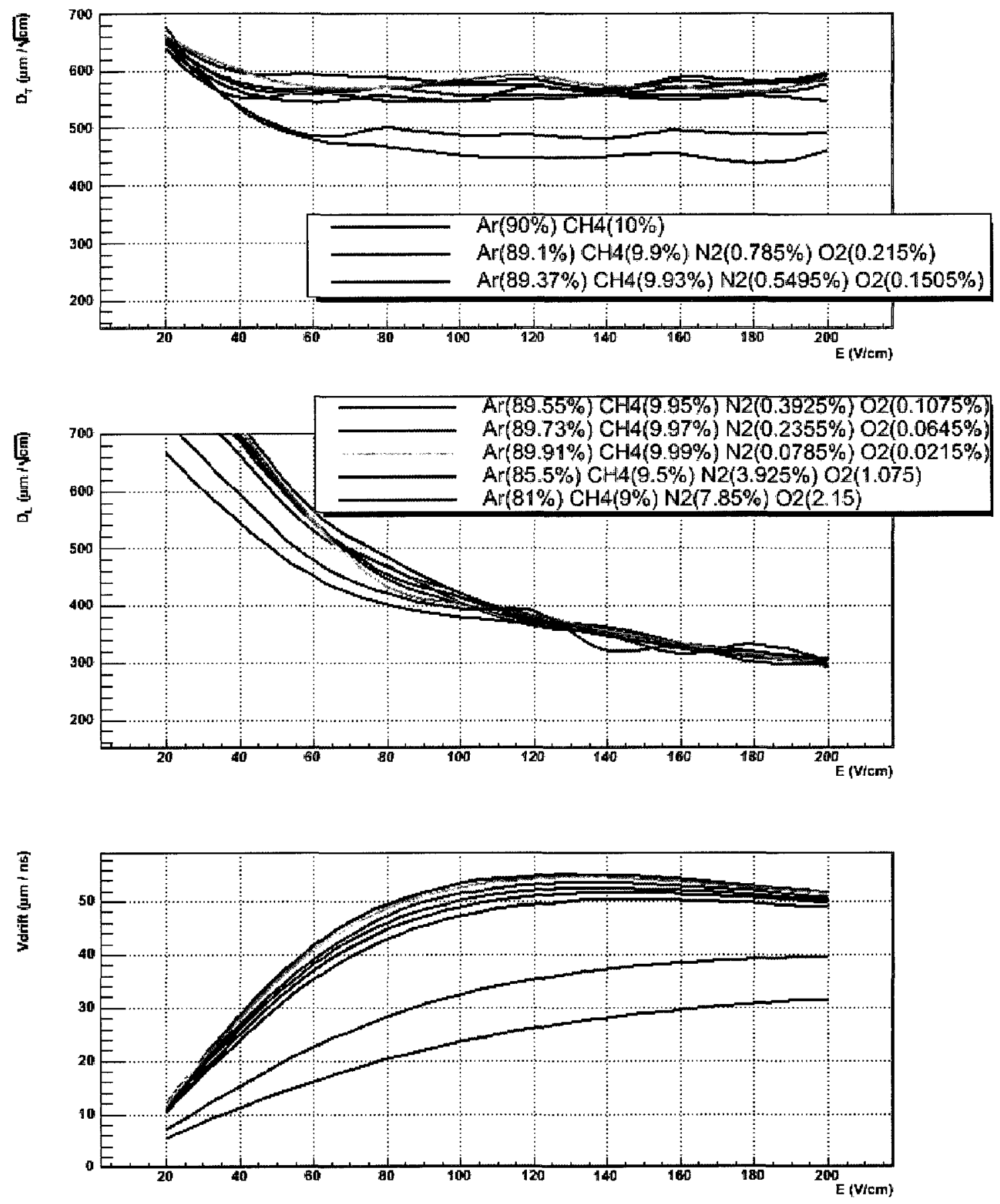

Figure B.1: MAGBOLTZ results for argon-methane contaminated with air. Top: Transverse diffusion. Middle: Longitudinal diffusion. Bottom: Drift velocity 

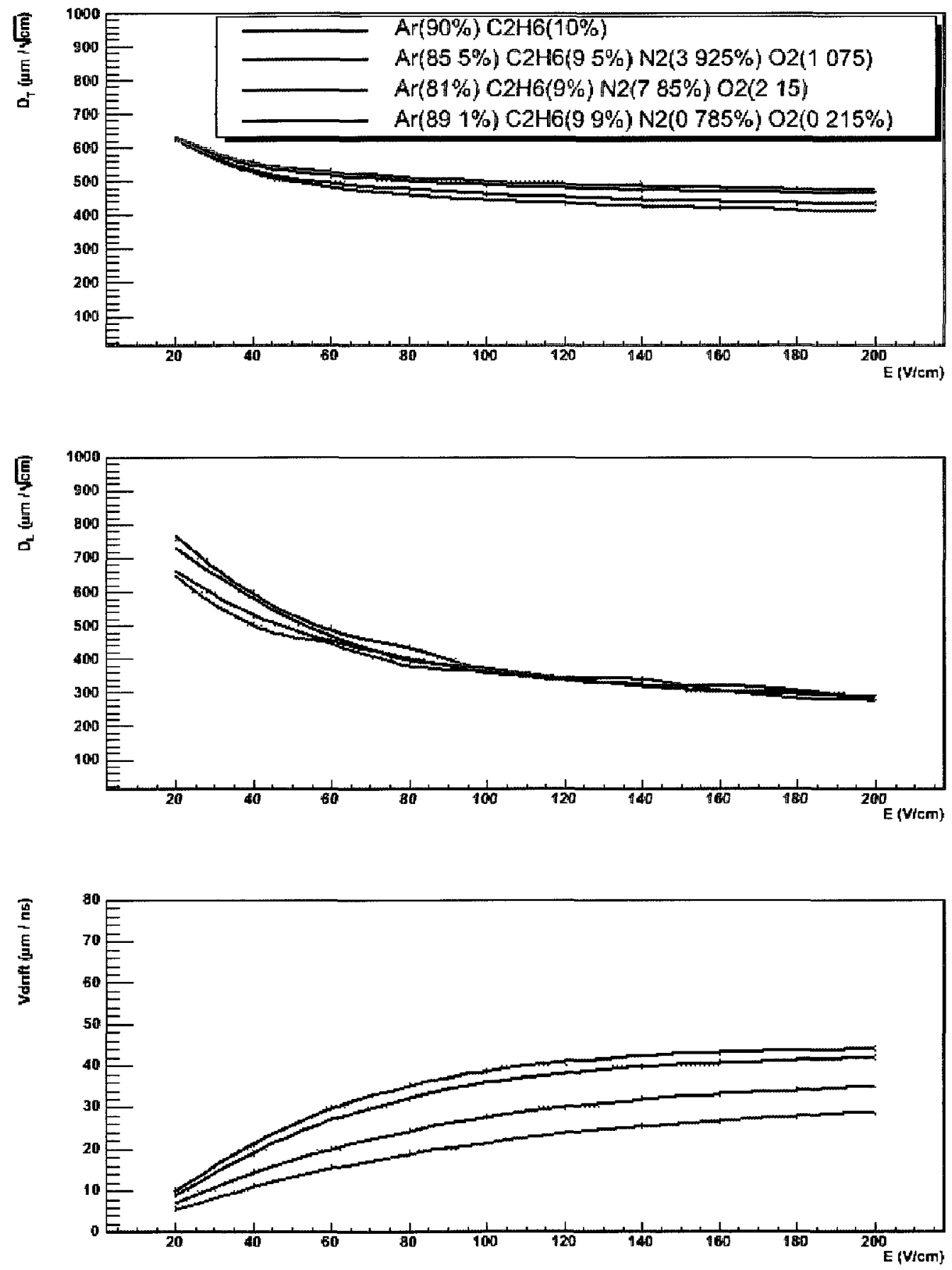

Figure B.2: MAGBOLTZ results for argon-ethane contaminated with air Top Transverse diffusion Middle Longitudinal diffusion Bottom Drift velocity 

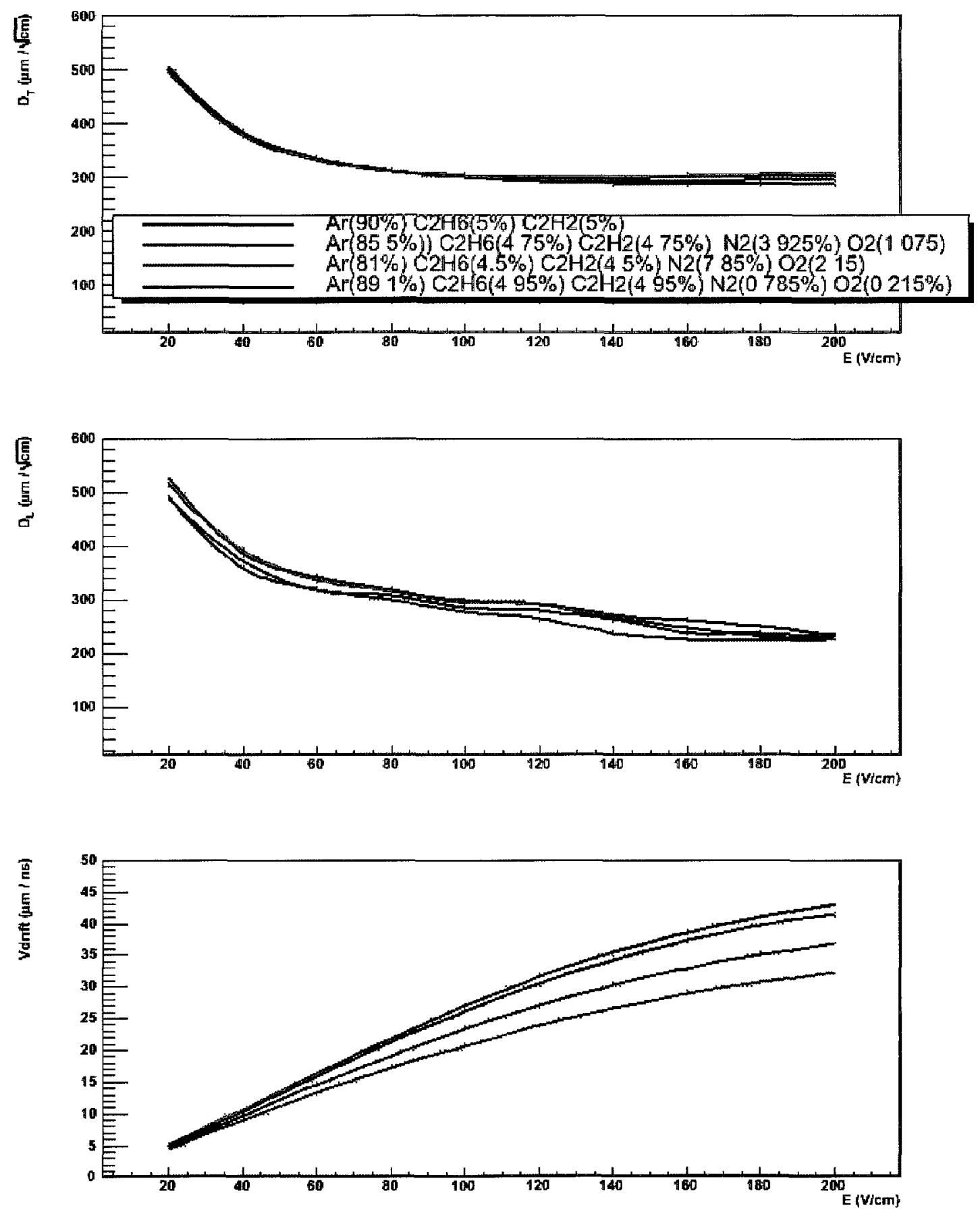

Figure B.3: MAGBOLTZ results for argon-ethane-acetylene contaminated with arr Top Transverse diffusion Middle Longitudinal diffusion Bottom Drift velocity 


\section{B.4 Ar- $\mathrm{CO}_{2}$}

The MAGBOLTZ results for argon-carbon dioxide contaminated with air are shown in fig B.4. This was done simply for completeness as the performance of the gas under various conditions is always beneficial to know. This was again done for the same concentrations of air as seen previously. Interestingly the air, even at concentrations of $5 \%$ and $10 \%$ do not significantly effect the drift velocity. Interestingly in this case it actually seems to increase the drift velocity slightly at a field of $105.2 \mathrm{~V} / \mathrm{cm}$.

\section{B.5 Conclusion}

The effects of air contamination depend significantly on the gas mixture in question. That is to say that the faster gases are more readily slowed by air than the slower ones. Moreover, the effects of significantly smaller concentrations of water vapour are much more substantial than even large concentrations of air. With that it can be said that should humid air leak into the detector the contribution from the water vapour is much more important than that from the air.

This is summarized in table B.1.

\begin{tabular}{cccc}
\hline \hline Gas & $\mathrm{V}_{0 \%}$ & $\mathrm{~V}_{1 \% \text { water }}$ & $\mathrm{V}_{10 \% \text { arr }}$ \\
$\mathrm{Ar}-\mathrm{CH}_{4}$ & 54 & 9 & 25 \\
$\mathrm{Ar}-\mathrm{C}_{2} \mathrm{H}_{6}$ & 39 & 8 & 22 \\
$\mathrm{Ar}-\mathrm{C}_{2} \mathrm{H}_{6}-\mathrm{C}_{2} \mathrm{H}_{2}$ & 27 & 8 & 22 \\
$\mathrm{Ar}-\mathrm{CO}_{2}$ & 4.5 & 3 & 4.8 \\
\hline \hline
\end{tabular}

Table B.1: Summary of conamination results for water and air in all gases. The values of drift velocity are quotes from the MAGBOLTZ plots at a drift field of 105.2 $\mathrm{V} / \mathrm{cm}$. The units of drift velocity are $\mu \mathrm{m} / \mathrm{ns}$. 

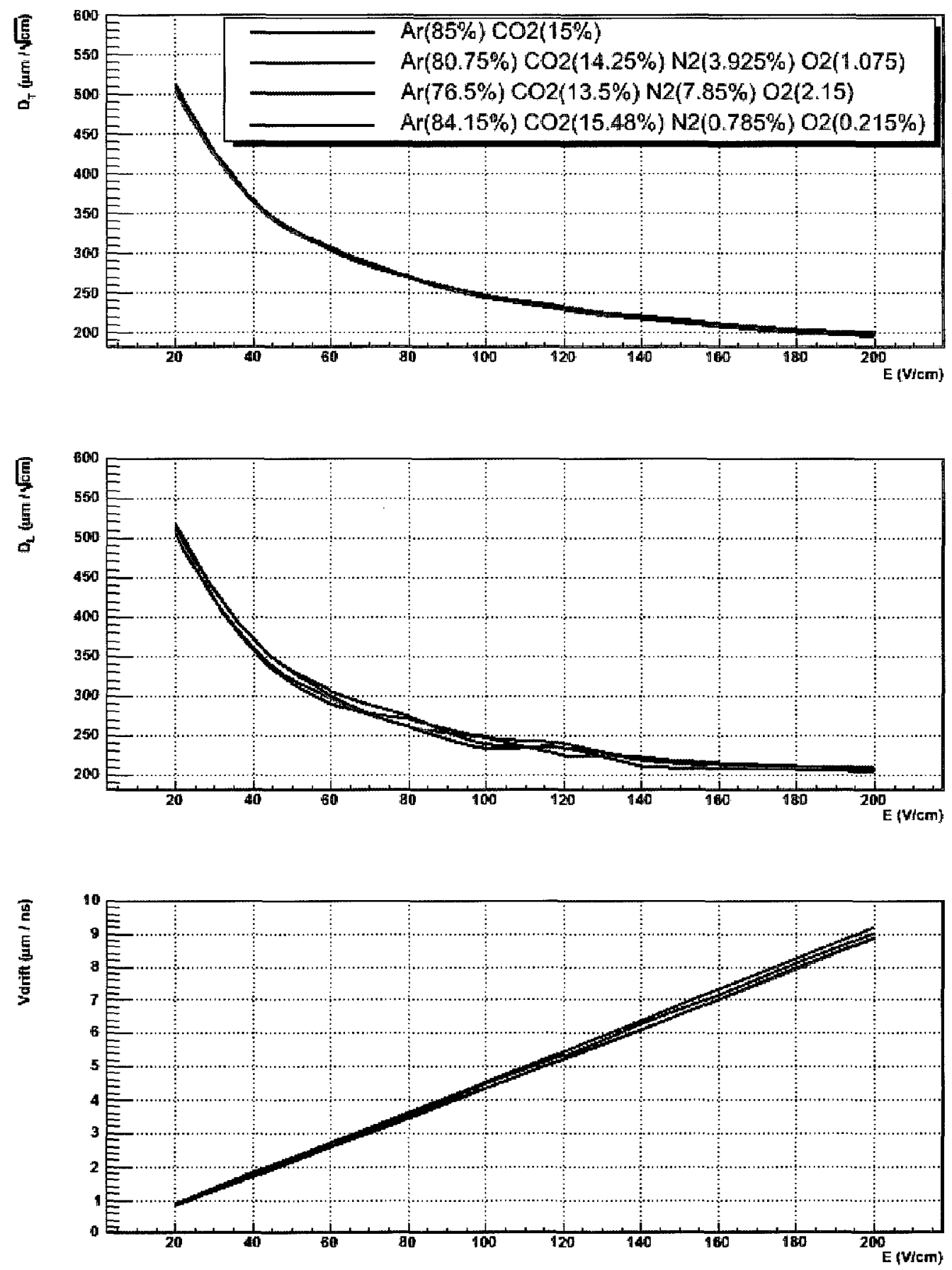

Figure B.4: MAGBOLTZ results for argon-carbon dioxide contaminated with air. Top: Transverse diffusion. Middle: Longitudinal diffusion. Bottom: Drift velocity 


\section{Appendix C}

\section{Humidity}

\section{C.1 Relative Humidity}

The concept of relative humidity is very straightforward. It is a measure of the amount of water vapour air can contain at a particular temperature. It is stated concisely in eqn C.1 where the the symbol $\rho$ represents the density in question.

$$
\text { RelativeHumidity }=\frac{\rho_{\text {observed }}}{\rho_{\text {saturation }}} 100 \%
$$

Once the fraction of vapour pressure of water reaches the saturation level of air at that temperature the moisture condenses and appears as dew. The saturation vapour density of air depends on temperature. That is to say that at lower temperatures it becomes saturated with smaller amounts of water vapour than at higher temperatures. This is important to keep in mind as a high relative humidity during the winter months does not equate to the same relative humidity in summer months having the same mass of water per unit volume air. In short, air during the summer months contains, by mass, more water vapour than during the winter.

The humidity data found in [27] are plotted in fig C.1.

The mean temperature for the very same months in Ottawa as found in [28] is 


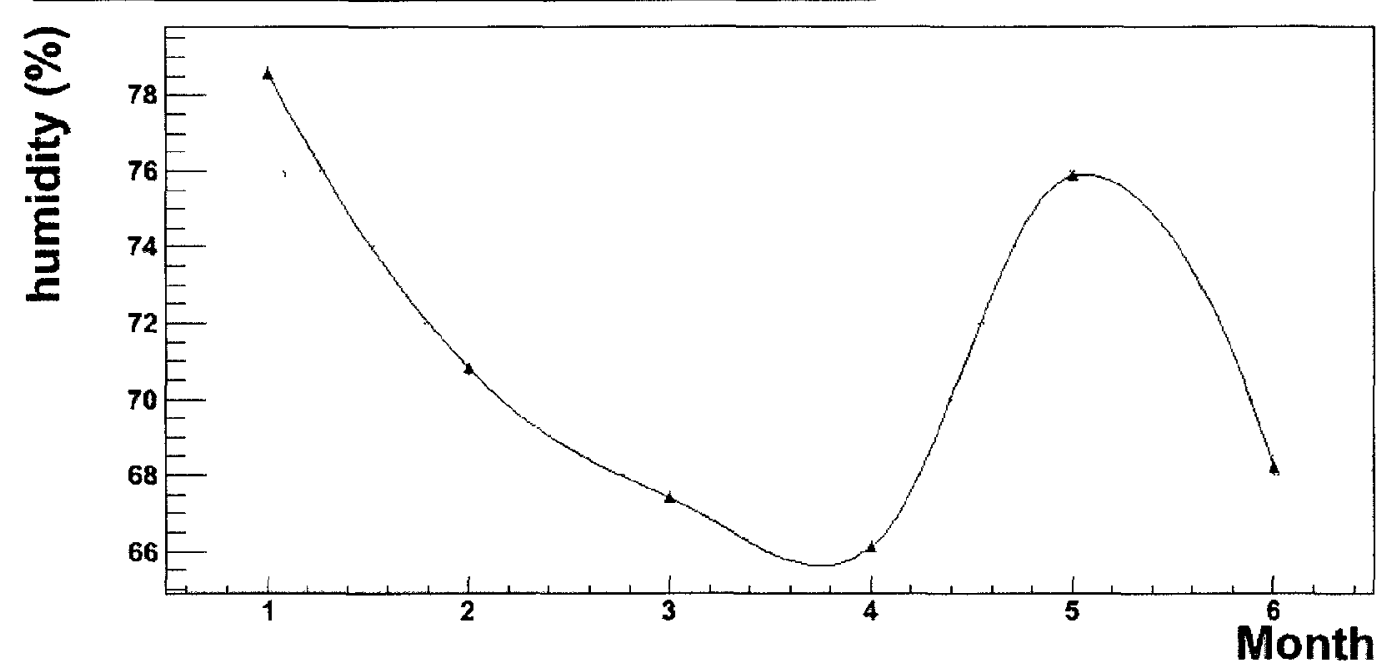

Figure C.1: Relative humidity from Jan. 2011 to June 2011

shown in fig C.2. Despite the relative humidity only fluctuating slightly over the months this experiment was carried out at the mean temperature, and therefore the saturation vapour pressure of air, increased significantly. 


\section{Mean temperature for Ottawa from Jan. to June 2011}

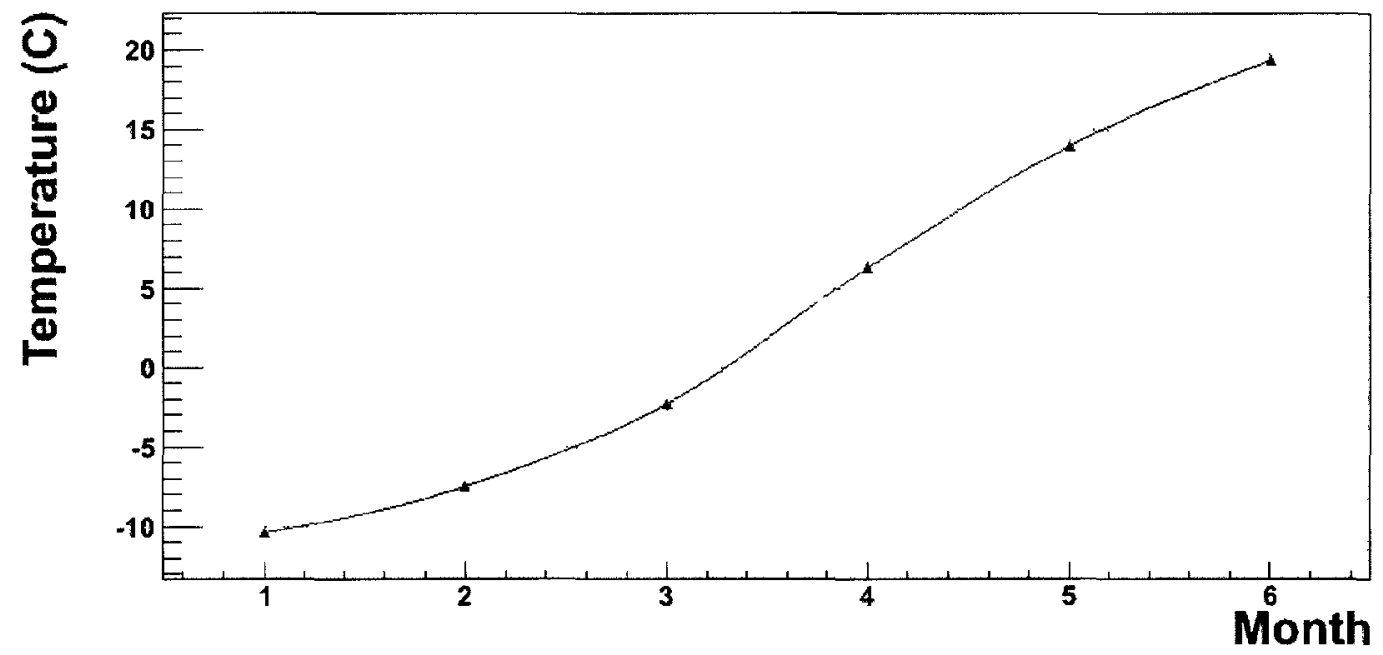

Figure C.2: Mean temperature from Jan 2011 to June 2011 